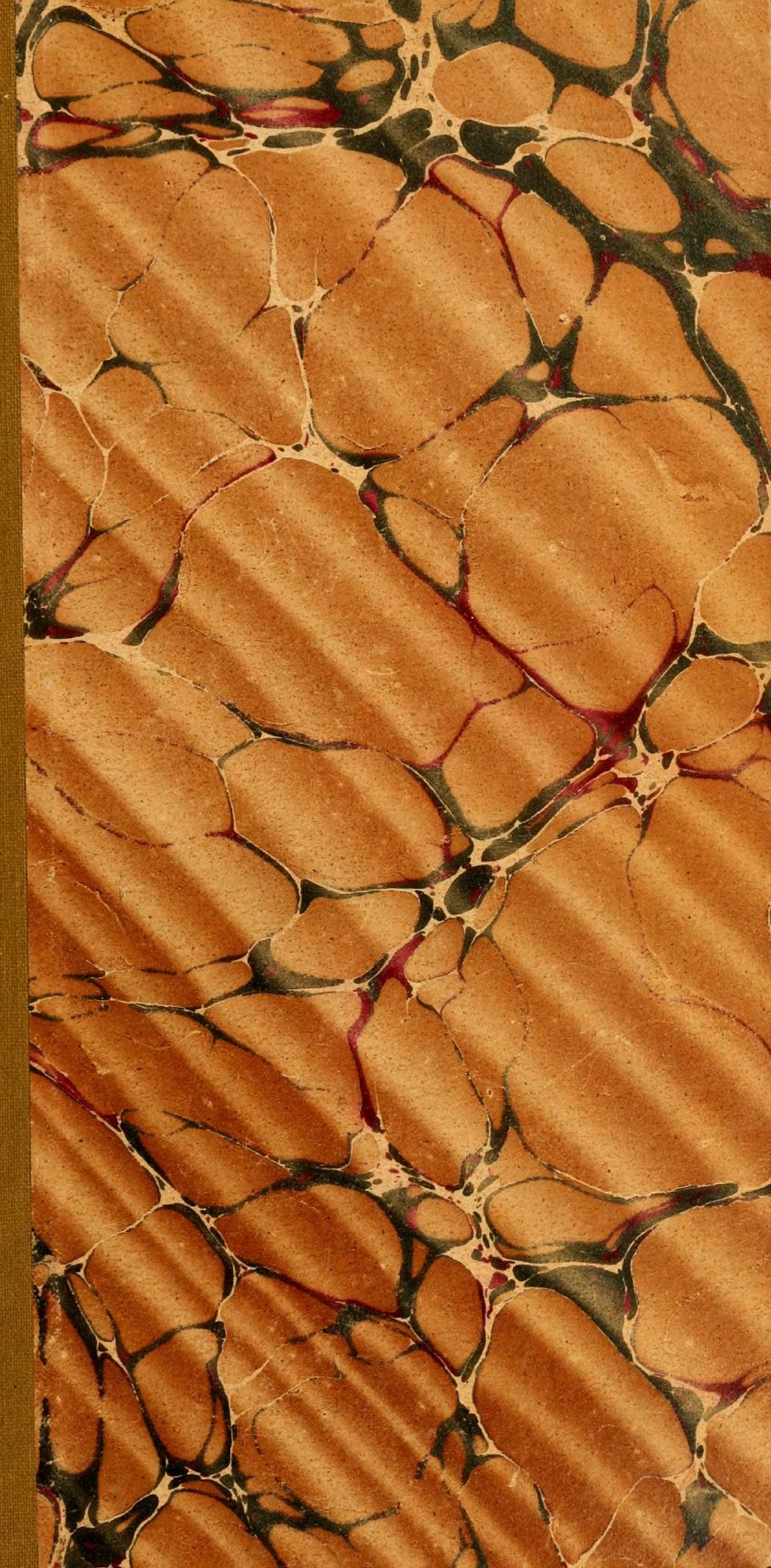


$P_{z}-L 847$

Bound 1938

\section{HARVARD UNIVERSITY.}

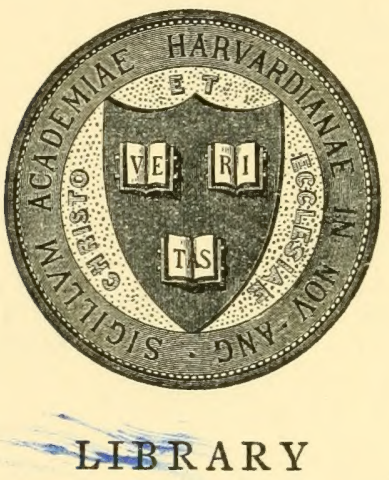

OF THE

MUSEUM OF COMPARATIVE ZOÖLOGY

$Y^{4}, 464$

GIFT OF

Harvard College hibrary

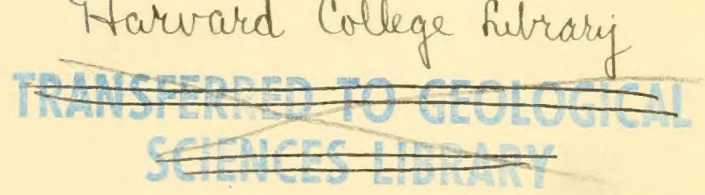




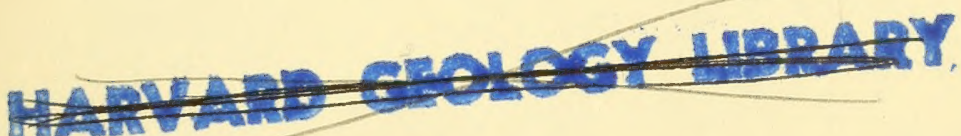
in 



\author{
$7 \%, 464$ \\ C A T A L O G U E
}

OF THE

\title{
CAMBRIAN AND SILURIAN
}

F O S S I L S

IN THE

\section{MUSEUM OF PRACTICAL GEOLOGY.}

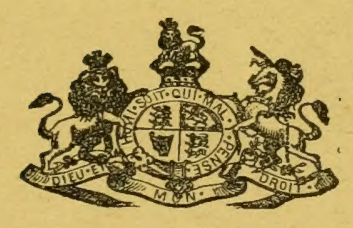

LONDON :

SOLD AT MUSEUM.

Printed for Her Majesty's Stationery Office,

BY GEORGE E. EYRE AND WILIIAM SPOTTISWOODE, PRINTERS TO THE QUEEN'S MOST EXCELLENT MAJUSTY.

1878 .

Price 2s. $6 d$. 



\section{A T A L O G U E}

OF THE

\section{CAMBRIAN AND SILURIAN}

\section{F O S S I L S}

IX THE

\section{Ỹ. Prit. - Yeol purvey -}

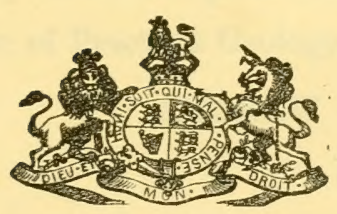

LONDON :

SOLD AT MUSEUM.

Printed for Her Majesty's Stationery Office, BY GEORGE E. EXRE AND WILLIAM SPOTTISWOODE, PRINTERS TO THE QUEEN'S MOST EXCELLENT MAJESTY. 1878.

$$
\text { Price 2s. 6d. }
$$


Ser 2490.62

\section{Pierce fund}

This Catalogue has been drawn up by Mr. E. T. Newton, Assistant Naturalist, under my superintendence.

The Specimens have been named by Mr. Etheridge, F.R.S., Palæontologist to the Geological Survey of Great Britain.

T. H. HUXLEY.

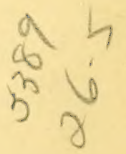


Srnce the publication of the previous edition of the Catalogue the collection of Silurian Fossils has been increased by nearly 3,000 specimens. To a large extent this is due to the munificent gift of the Rev. E. Wyatt-Edgell, whose son, the late Lieutenant H. A. Wyatt-Edgell, had during several years and at great expense made a collection of nearly 4,000 specimens of British Palæozoic Fossils, all of which were presented to this Museum In accordance with an expressed wish, these fossils are distinguished by a printed label "Wyatt-Edgell Collection," so that they remain as a permanent record of his work.

This series having now been incorporated with those previously in the cases, there is probably no collection of Silurian Fossils of any country more complete than that which is exhibited in the Museum of Practical Geology.

\section{ANDREW C. RAMSAY,}

Director-General. 



\section{CAIIBRIAN AND SILURLIN FOSSILS.}

INDEX.

ARRANGED STRA'TIGRAPHICALLY.

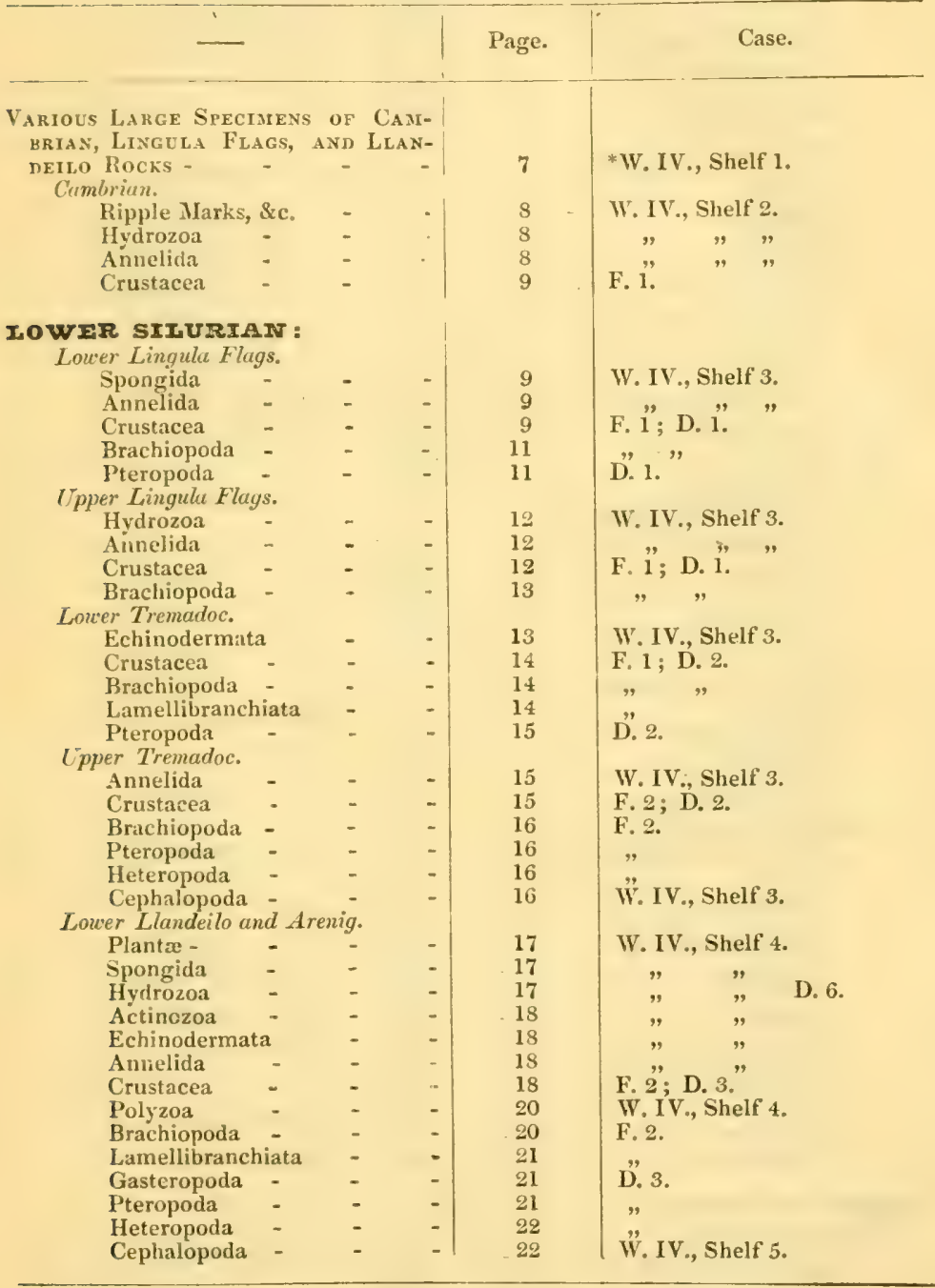

*W. $=$ Wall Case, F. $=$ Flat Case, $D_{0}=$ Drawer. 


\begin{tabular}{|c|c|c|c|c|c|}
\hline & & & & Page. & Case. \\
\hline \multirow{2}{*}{\multicolumn{6}{|c|}{$\begin{array}{l}\text { IOWER SIIURIAI-cont. } \\
\text { Upper Llandeilo. }\end{array}$}} \\
\hline & & & & & \\
\hline Plantie & - & - & - & 22 & WV. IV., Shelf 5. \\
\hline Hydrozoa & - & - & - & 23 & " $\quad$ D. 7. \\
\hline Actinozoa & - & - & - & 25 & W. IV., Shelf 6. \\
\hline Echinodermat: & & - & - & 25 &,$\quad$, \\
\hline Anuelida & - & - & - & 25 & $" \quad$ " \\
\hline Crustacea & - & - & - & 25 & F. 3; D. 4" \\
\hline Polyzoa & - & - & - & 28 & W. IV., Shelf 6. \\
\hline Brachiopoda & - & - & - & 28 & F. 3 ; D. 5 . \\
\hline Lamellibranch & liata & - & - & 29 & F. 3 . \\
\hline Gasteropoda & - & - & - & 29 & D. 5 . \\
\hline Pteropoda & - & - & - & 29 & , \\
\hline Heteropoda & - & - & - & 29 & 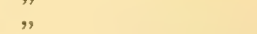 \\
\hline \multicolumn{6}{|l|}{ Caradoc. } \\
\hline Various large & speci & ens & - & 30 & W. V., Shelf 1. \\
\hline Plauta & - & - & - & 30 & , $\quad, \quad 2$. \\
\hline Spongida & - & - & - & 31 & ",$\quad$, \\
\hline Hydrozoa & - & - & - & 31 & , , \\
\hline Actinozoa & - & - & - & 32 & " $\quad 2 \quad 2 \& 3$ \\
\hline Echinodermat & & - & - & 34 & , $3 \& 4$ \\
\hline Annelida & - & - & - & 36 & $" \quad \quad 4$ \\
\hline Crustacea & - & - & - & 36 & F. $4,5,6,7$ \\
\hline Polyzoa & - & - & - & 44 & W. V., Shelf 4, 5 . \\
\hline Brachiopoda & - & .. & - & 45 & F. $7,8,9 ;$ D. 8 \\
\hline Lamellibranch & liata & - & - & 52 & F. 9,10 \\
\hline Gasteropoda & - & - & - & 54 & F. 10,11 \\
\hline Pteropoda & - & - & - & 56 & F. 11 . \\
\hline Heteropoda & - & - & - & 57 & m, \\
\hline Cephalopoda & - & - & - & 58 & W. V., Shelf 5, 6 . \\
\hline \multicolumn{6}{|c|}{ Pebble Beds, Budleigh Salterton. } \\
\hline Echinodermat & & - & - & 60 & D. 10 . \\
\hline Annelida & - & & - & 60 & , \\
\hline Crustacea & - & - & - & 60 & $"$ \\
\hline Brachiopoda & - & - & - & 60 & $"$ \\
\hline Lamellibranch & hiata & - & - & 61 & ", \\
\hline \multirow{2}{*}{\multicolumn{6}{|c|}{$\begin{array}{l}\text { Cephalopoda - } \\
\text { Pelble Beds, other localities. }\end{array}$}} \\
\hline & & & & & \\
\hline Actinozoa & - & - & - & 61 & D. 11 . \\
\hline Echinodermat & & - & - & 61 & ", \\
\hline Annelida & - & - & - & 61 & $"$ \\
\hline Brachiopoda & - & - & - & 61 & ", \\
\hline Lamellibranch & hiata & - & - & 61 & $"$ \\
\hline \multicolumn{6}{|c|}{ Lower Llandovery. } \\
\hline Virrious large & speci & & - & 62 & W. VI., Shelf 1. \\
\hline Planta - & - & - & $=$ & 62 & $, \quad, \quad 2$. \\
\hline Spongida & - & - & - & 62 & $" \quad, \quad " \quad$, \\
\hline Actinozoa & - & - & - & 62 & $" \quad, \quad "$ \\
\hline Echinodermat & & - & - & 63 & ", \\
\hline Annelida & - & - & - & 63 & = \\
\hline Crustacea & - & - & $=$ & 63 & F. 12. \\
\hline Polyzoa & - & - & -1 & 64 & W. VI., Shelf 2. \\
\hline Brachiopoda & - & - & - & 64 & F. 12. \\
\hline \multicolumn{6}{|c|}{ Lamellibranchiata } \\
\hline Gasteropoda & - & - & - & 66 & F. 13. \\
\hline Pteropoda & - & - & - & 67 & , \\
\hline Heteropoda & - & - & - & 67 & " Sholf 8 \\
\hline Cephalopoda & - & - & - & 67 & WV. VI., Shelf 2. \\
\hline $\begin{array}{r}\text { UPPER SIIURIA } \\
\text { Upper Llandovery. }\end{array}$ & $\mathbf{N}:$ & & & & \\
\hline Various large & speci & ens & - & 68 & W. VI., Shelf 1. \\
\hline Planta - & - & - & - & 69 & , $\quad 3$. \\
\hline Spongida & - & - & - & 69 & $"$ \\
\hline Hydrozoa & - & - & -1 & 69 & $"$, \\
\hline
\end{tabular}


Page.

Case.
UPPER SIZURIAN-cont.

Upper Llandovery-cont. Actinozoa -
Annelida -

$\begin{array}{lll}\text { Annelida } & - \\ \text { Crustacea } & - & -\end{array}$

Polyzoa -

Brachiopoda

Gasteropoda -

Pteropoda

Heteropoda

Cephalopora

Woolhope Beds.

Spongida

Actinozon

Echinodermata

Crustacea

Brachiopoda

Lamellibranchiata

Gasteropoda

Heteropoda .

Cephalopoda

Wenlock shale.

Plantæ -

Hydrozoa

Actinozoa

Echinodermata

Annelida

Crustacea

Polyzoa

Brachiopoda.

Lamellibranchiata

Gasteropoda

Pteropoda

Heteropoda

Cephalopoda

Wenlock Limestone.

Various large specimens

Spongida

Hydrozoa

Actinozoa

Echinodermata

Annelida

Crustacea

Polyzoa

Brachiopoda

Lamellibranchiata

Gasteropoda -

Pteropoda

Heteropoda

Cephalopoda

Lower Ludlow.

Various large specimens

Plantae

Spongida

Hydrozoa

Actinozoa

Echinodermata

Annelida

Crustacea

Polyzoa
W. VI., Shelf $3 \& 4$.

F. 13

W. VI., Shelf 4 .

F. 13,14 ; D. 14 .

F. 14,15 ; D. 14 .

.F. 15; D. 14 .

F. 15 .

W". VI., Shelf 4.

W. VI., Shelf 5 .

F. 15 .

F. 15,16 .

F. 16 .

W. VI., Shelf 5.

W. VI., Shelf 1. ,, 5 . 5 .

W. "VI., Shelf" 6; W. VIIIs. Shelf 1,2 ; and W. VIIIB. W. VI., Shelf 6 .

F. 16,$17 ;$ D. 15 ; W. VI., Shelf 6.

W. VI., Shelf 6 .

F. 17,$18 ;$ D. 15 .

F. $18 ;$ D. 15 .

D. 15 .

W". VI., Shelf 6 .

f W. VII., Shelf 1 .

$\{$ W. VIII., Shelf $1,2,3$.

W. VII., Shelf 2.

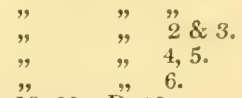

F. 19, 20; D. 19 .

IV. VII., Shelf 6.

F. 20,$21 ;$ D. 19,20 .

F. $22 ;$ D. 21 .

," ,

W. 'VII., Shlielf 6.

W. IX., Shelf 1.

$$
\begin{aligned}
& \text {, }, 2 . \\
& \text {, " " } \\
& \text {, ," , } \\
& \text {," } \quad \text {," } \quad 2 \& 3 . \\
& \text {," } 3 .
\end{aligned}
$$

F. 23 ; D. 22; W. IX., Shelf 3 , and $W . X$.

W. IX., Shelf 3. 


\begin{tabular}{|c|c|c|c|c|c|}
\hline & & & & Page. & Case. \\
\hline \multicolumn{6}{|c|}{$\begin{array}{l}\text { UPPIR SIIURIAIN-cont. } \\
\text { Lower Ludlow-cont. }\end{array}$} \\
\hline Brachiopoda & - & - & - & 119 & F. $23 ;$ D. 22. \\
\hline Lamellibranchi & liata & - & - & 120 & F. 23,$24 ;$ D. 22 . \\
\hline Gasteropoda & - & - & - & 121 & F. $24 ;$ D. 22 \\
\hline Pteropoda & - & - & - & 122 & WV. IX., Shelf 3 ; F. 24 ; D. 23. \\
\hline Heteropoda & - & - & - & 123 & F. $24 ;$ D. 23 . \\
\hline Cephalopoda & - & - & - & 123 & W. IX., Shelf 3, 4. \\
\hline $\begin{array}{c}\text { Aymestry Limestone } \\
\text { Planta - }\end{array}$ & $e$. & - & - & 124 & W. IX., Shelf $t$. \\
\hline Spongida & - & - & - & 124 &,$\quad$, \\
\hline Actinozoa & - & - & - & 125 & , 5. \\
\hline Crustacea & - & - & - & 125 & F. 25 \\
\hline Polyzoa & - & - & - & 125 & W. IX., Shelf 4 \\
\hline Brachiopoda & $\because$ & - & - & 125 & F. 25. \\
\hline Lamellibranch & liata & - & - & 126 & , \\
\hline Gasteropoda & - & - & - & 126 & $"$ \\
\hline Heteropoda & - & - & - & 126 & " \\
\hline $\begin{array}{l}\text { Cephalopora } \\
\text { Upper Ludlow. }\end{array}$ & - & - & - & 126 & W. IX., Shelf 4. \\
\hline Various large s & speci & ens & - & 127 & W. IX, Shelf 1. \\
\hline Plantæ & - & - & - & 127 & $\Rightarrow \quad 4 \quad 4$ \\
\hline Spongida & - & - & - & $128-$ & " $\quad$ " 5. \\
\hline Hydrozoa & - & - & - & 128 & ", , \\
\hline Actinozoa & - & - & - & 128 & $"$, \\
\hline Echinodermata & & - & - & 129 & $"$, \\
\hline Annelida & - & - & - & 129 & " " " " \\
\hline Crustacea & - & - & - & 130 and 141 & $\begin{array}{l}\text { F. } 26 \text {; D. } 24 ; \text { W. IX., Shelf } \\
5 \text {, and W. X. }\end{array}$ \\
\hline Polyzoa & - & - & - & 131 & W. IX., Shelf 6. \\
\hline Brachiopoda & - & - & - & 131 & F. 26,$27 ;$ D. 25 . \\
\hline Lamellibranch & liata & - & - & 133 & F. 27,$28 ;$ D. 25,26 . \\
\hline Gasteropoda & - & - & - & 137 & F. $28 ;$ D. 26 . \\
\hline Pteropoda & - & - & - & 139 & $\Rightarrow$ \\
\hline Heteropoda & - & - & $\cdot$ & 139 & " \\
\hline Cephalopoda & - & - & - & 140 & W. IX. Shelf 6. \\
\hline
\end{tabular}




\section{INDEX TO MIUSEUM CASES.}

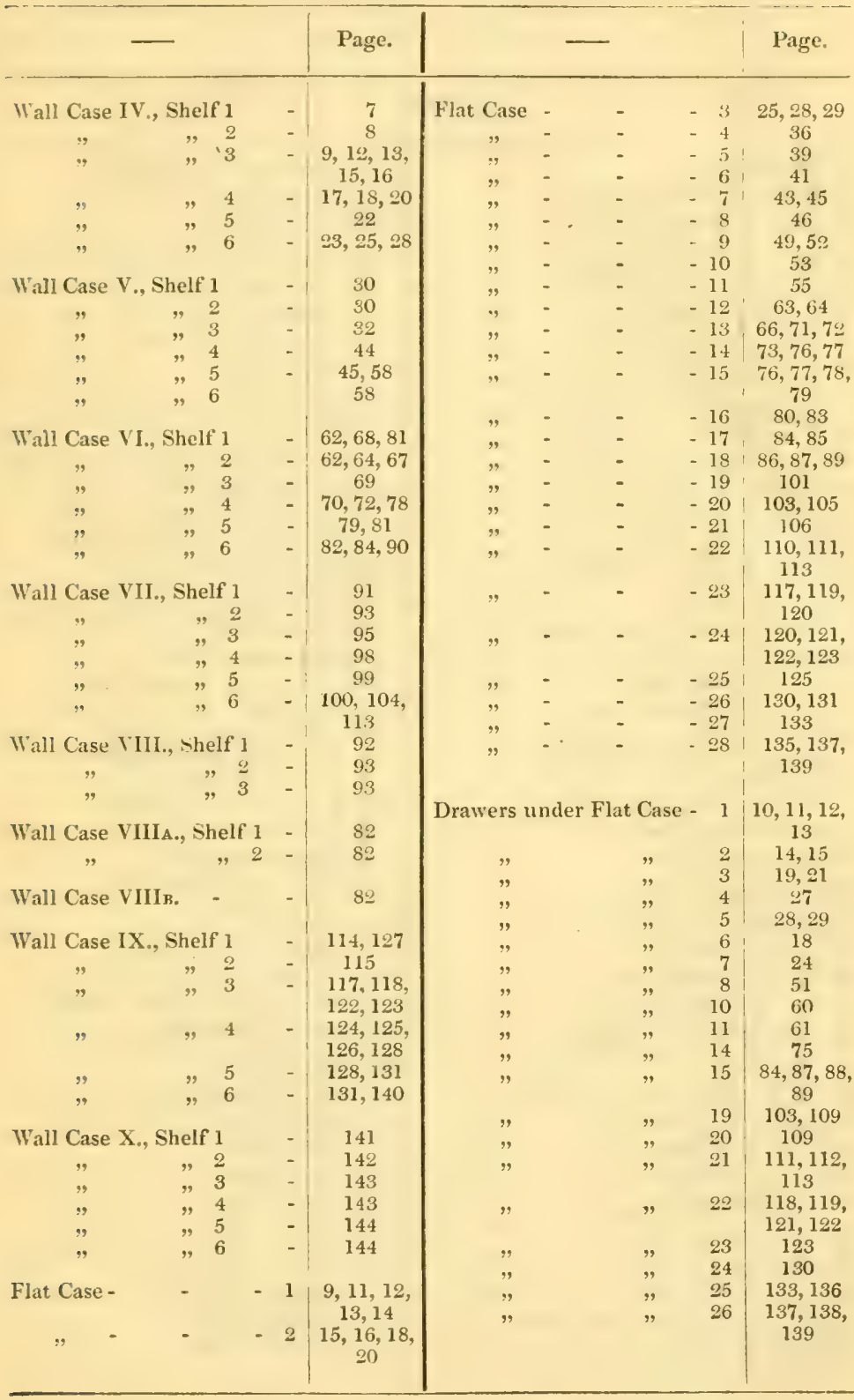




\section{DONORS OF CAMIBRIAN AND SILCRIAN FOSSILS.}

The numbers before the names are referred to in the fourth columns of the Catalogue.

1. Dr. Kinahan.

2. Bryce M. Wright, Esq.

3. Prof. Harkness, F.R.S.

4. C. W. Peach, Esq.

5. Sir R. 1. Murchison, Bart.

6. C. Moore, Esq., F.G.S.

7. -. Lloyd, Esq.

8. Prof. Macdonald, F.G.S.

9. J. W. Salter, Esq., F.L.S.

10. Prof. T. MIcK. Hughes, M.A.

11. Prof. J. Nicol, F.R.S.E.

12. R. Lightbody, Esq., F.G.S.

13. I. D. Darbishire, B.A.

14. - Davies, Esq.

15. Prof. $\mathrm{M}^{\circ} \mathrm{Coy}, \mathrm{F} . \mathrm{G} . \mathrm{S}$.

16. --. Prosser, Esq.

17. Prof. T. R. Jones, F.R.S.

18. Ii. Gibbs, Esq.

19. Dr. Horne.

20. Lieut. H. A. Wyatt-Edgell.

21. Earl Ducie, F.R.S.

22. -. Williams, Esq.

23. -. Collingwood, Esq.

24. Dr. Reiden.

25. Dr. R. B. Grindrod, F.G.S.

26. J. Bolton, Esq.

27. G. Maw, Esq., F.L.S.

28. C. Callaway, Esq., M.A.

29. J. Proud, Esq.

30. WV. Nichol, Esq.

31. Rev. J. Cumming.

32. --. Bright, Esq.

33. J. Gray, Esq.

34. Rev. Norman Glass.

35. - . Mushed, Esq.

36. Mrs. Elizabeth Warne.

37. Prof, J. Tennant, F.G.S.

38. Mrs. Day.

39. S. H. Blackwell, Esq., F.G.S.

40. Sir Wm. Guise, Bart.

41. Prof. Buckman, F.L.S.

42. W. J. Beckett, Esq.

43. C. Stokes, Esq.

44. Rev. F. Dyson, M.Á.

45. A. Marston, Esq.

46. C. R. Bayly, Esq.

47. F. S. B. Holt, Hisq.

48. G. W. Ormerod, Lisq., M.A.

49. J. Ruthven, Esq.

50. J. D. C. Sowerby, Esq.
51. R. Etheridge, Esq., F.R.S.

52. H. Hicks, Esq., F.G.S.

53. D. Homfray, Esq.

54. Dr. W. Ogle.

55. Rev. W. S. Symonds, M.A.

56. Rev. H. Hausman.

57. - Harrison, Esq.

58. Rev. P. B. Brodie, M.A.

59. G. Dawson, Esq.

60. R. Banks, Esq.

61. 'The Earl of Enniskillen, D.C.L.

62. J. Farie, Esq.

63. Manchester Geological Society.

64. E. H. Sheppard, Esq.

65. Sir Wyville Thompson, LL.D.

66. Matt. Moggeridge, Esq., F.G.S.

67. E. H. Birkenhead, Esq.

68. J. C. Moore, Esq., M.A.

69. Arthur Wyatt-Edgell, Esq., F.G.S.

70. J. Weaver, Esq.

71. G. B. Broome, Esq.

72. Rev. H. H. Winwood, M.A.

73. F. Ash, Esq.

7t. W. Vicary, Esq., F.G.S.

75. R. H. Valpy, Esq., F.G.S.

76. S. G. Perceval, Esq.

77. J. E. Marr, Esq., F.G.S.

78. R. Gill Goodwin, Esq.

79. 'T. Rowlandson, Esq.

80. Col. T. IV. Fletcher, M.A.

81. J. Gavey, Esq.

82. 'I. Gray, Esq.

83. H. Beckett, Esq., F.G.S.

84. G. E. Roberts, Esq.

85. Prof. J. Phillips, M.A., F.R.S.

86. 'The Earl of Cawdor.

87. Hugh Miller, Esq., F.G.S.

88. G. Cocking, Esq.

89. Rev. F. Crouch, B.D.

90. J. Harley, Esq.

91. - Clarke, Esq.

92. C. H. Wright.

93. A. Clark, Esq.

94. W. Davis, Esq.

95. J. Mushen, Esq.

96. David W. Roberts, Esq.

97. R. Slimon, Esq.

98. - . Smith, Esq.

99. J. E. Daris, Esq. 


\section{P A L EOZOIC D I V I I O N.}

LOWER PALAOZOIC SERIES.

CAMBRIAN AND LOWER SLLURIAN.

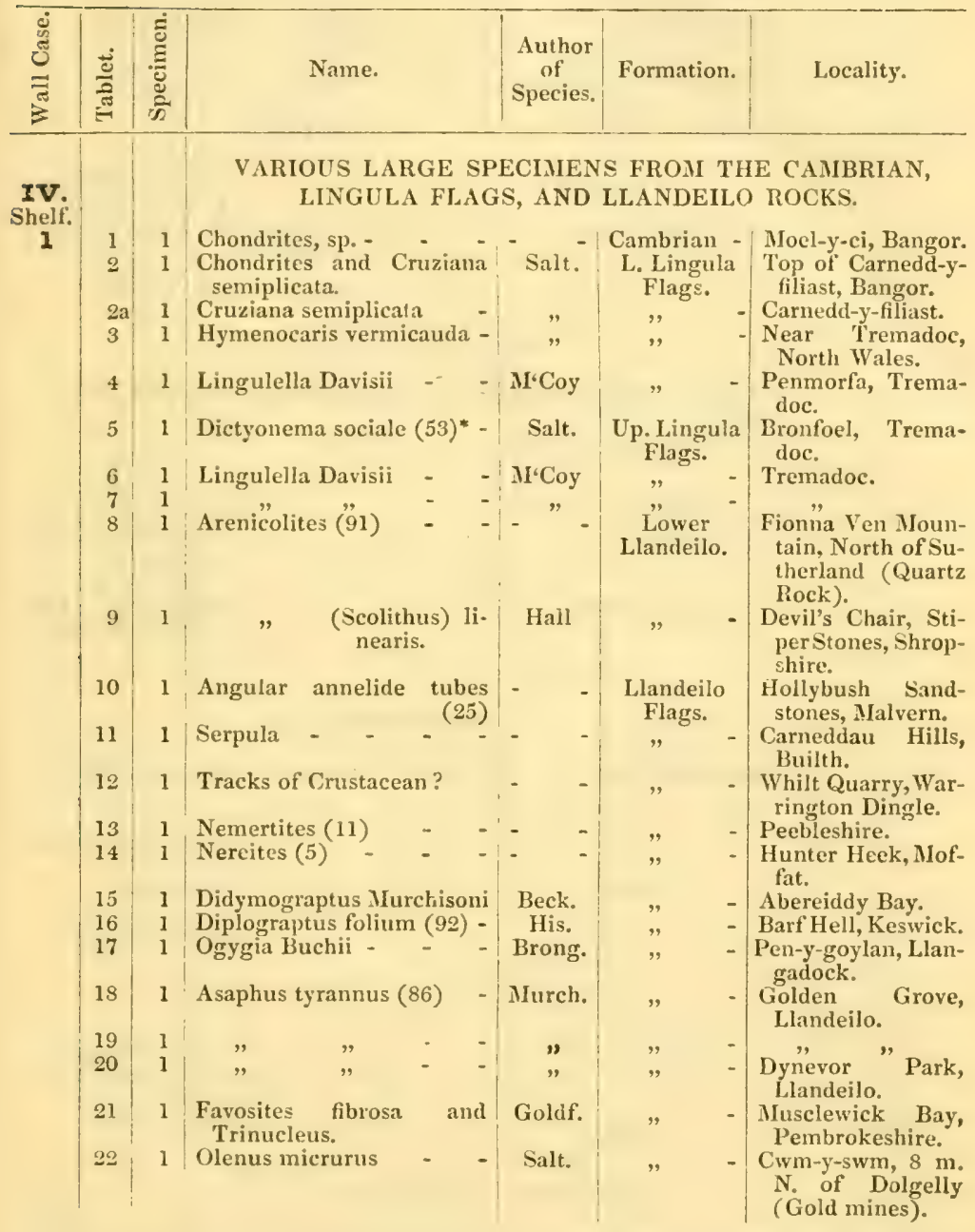

* These numbers refer to the List of Donors at page 6. 


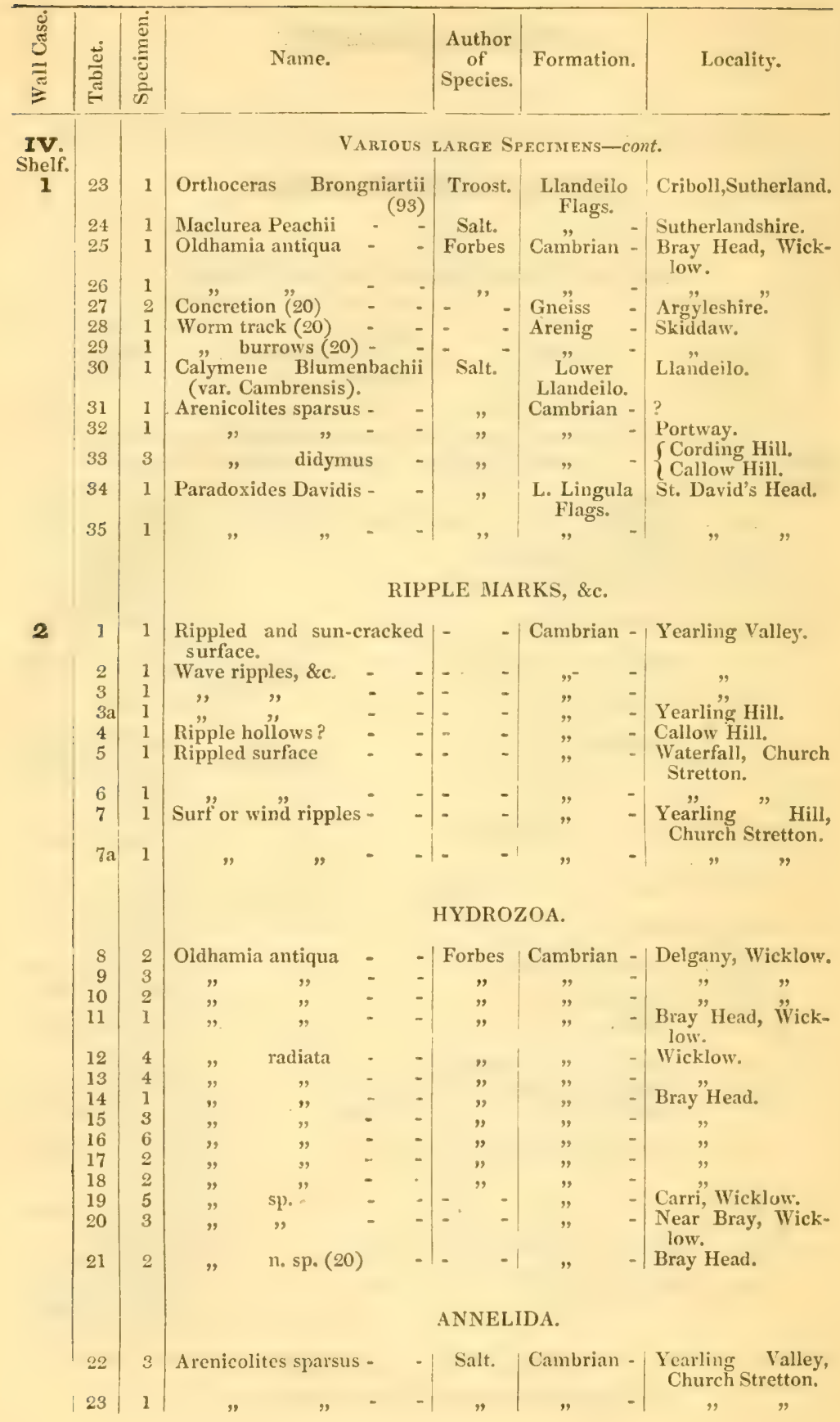




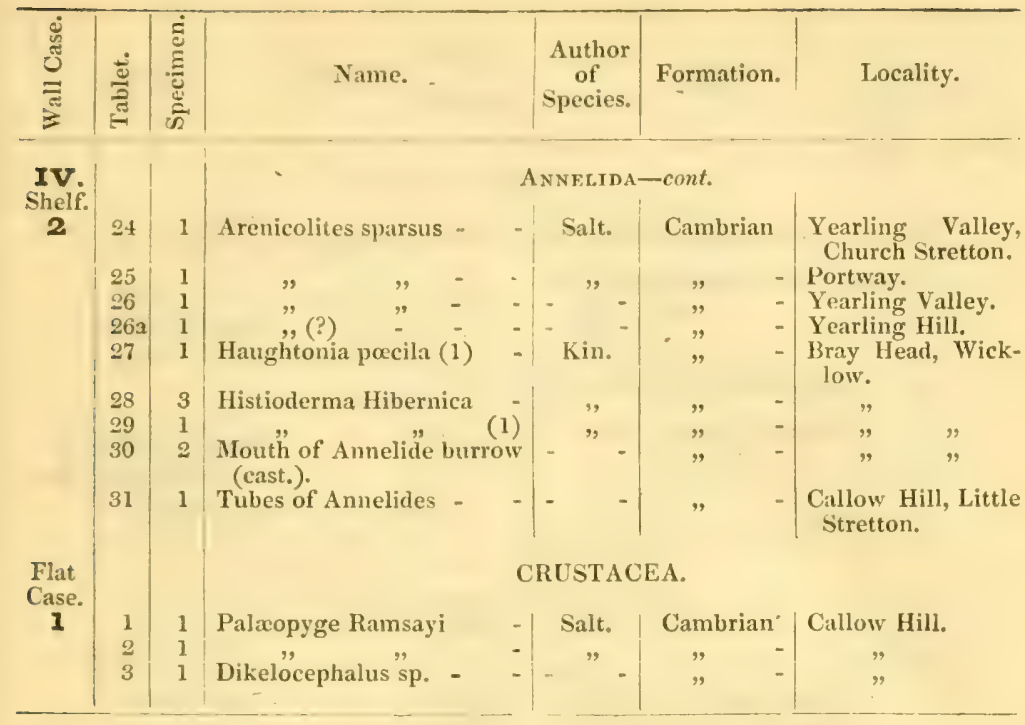

\section{LOWER SILURIAN.}

IOWER IINGUIA FIAGS.

\begin{tabular}{|c|c|c|c|c|c|c|}
\hline 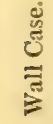 & 离 & 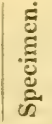 & Name. & $\begin{array}{l}\text { Author } \\
\text { of } \\
\text { Species. }\end{array}$ & Formation. & Locality. \\
\hline
\end{tabular}

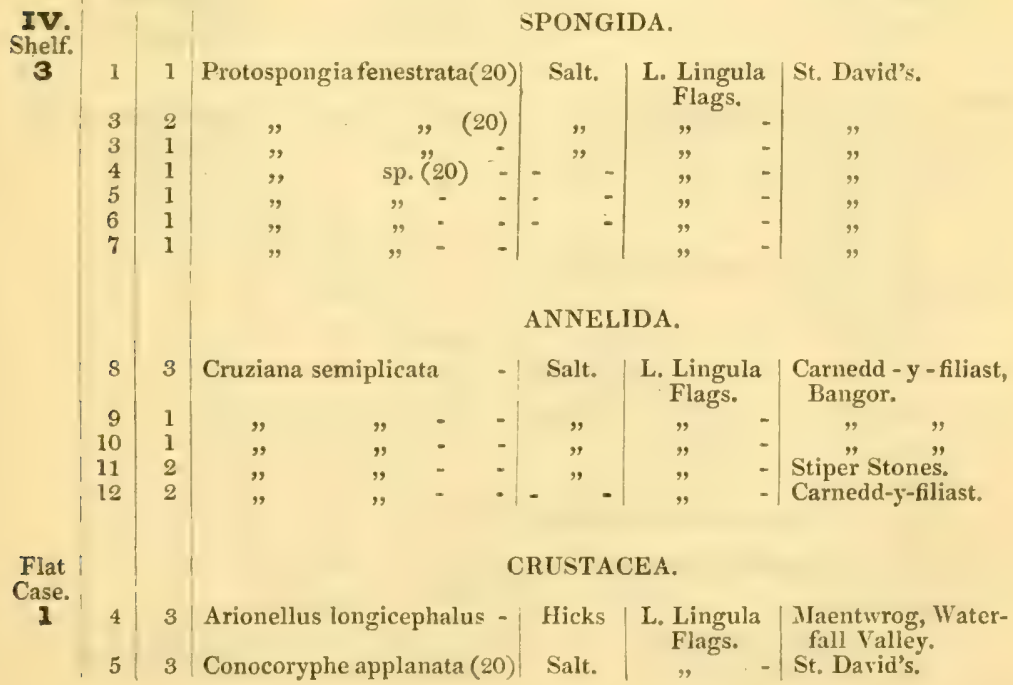




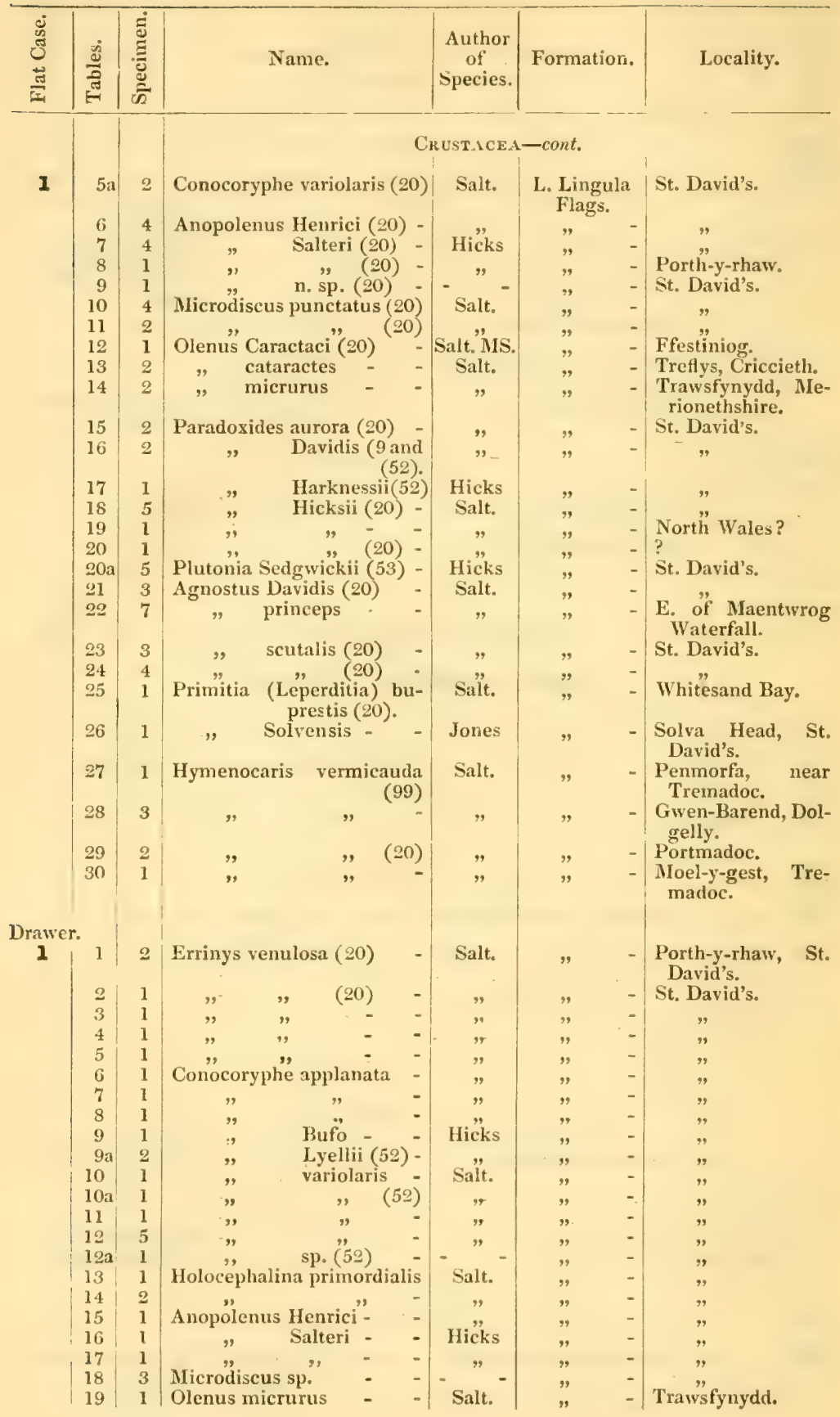




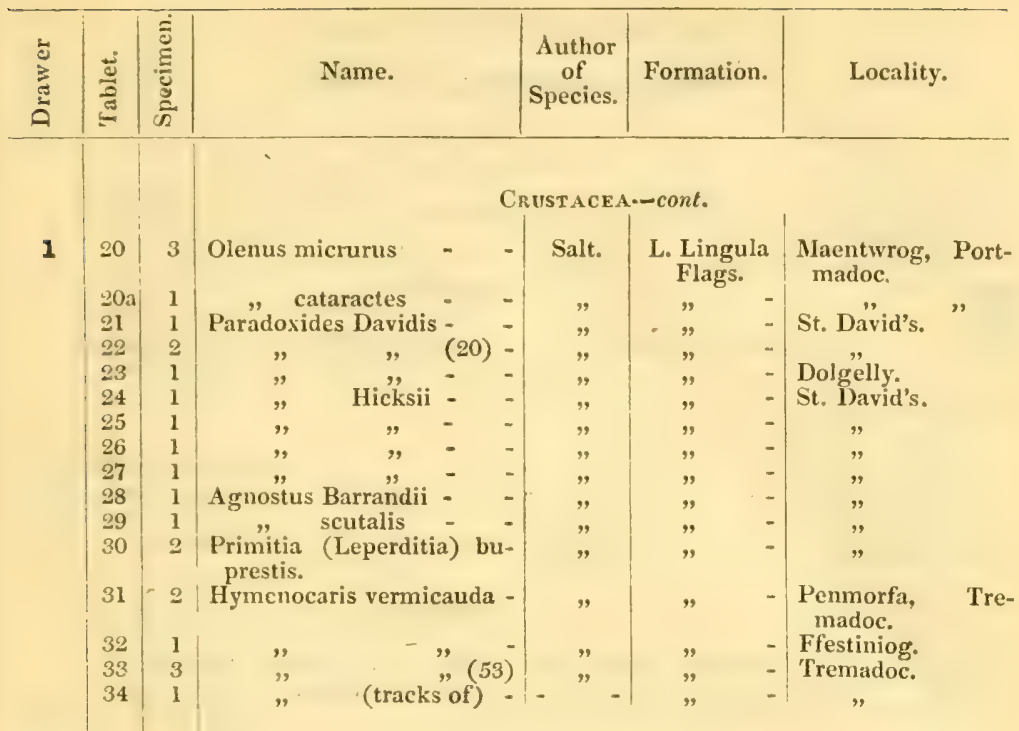

BRACHIOPODA.

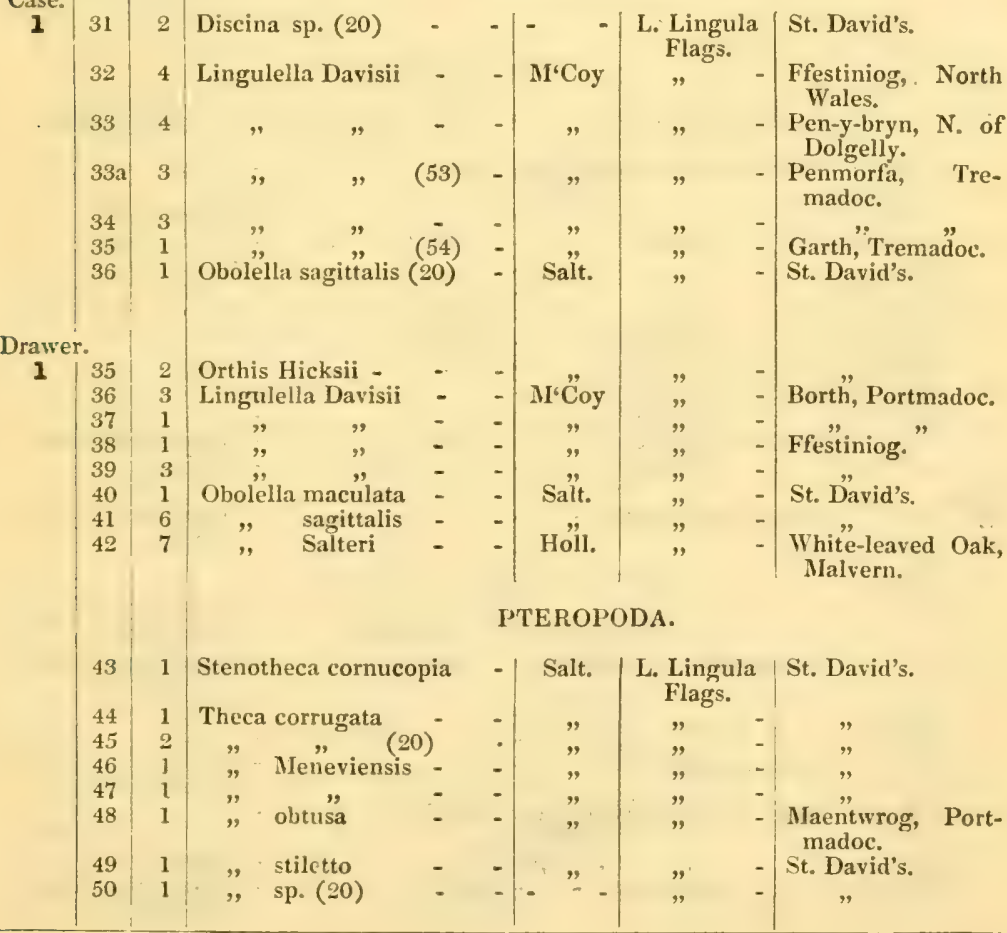




\section{LOWER SILURIAN-cont.}

UPPER IINGUIA FIAGS.

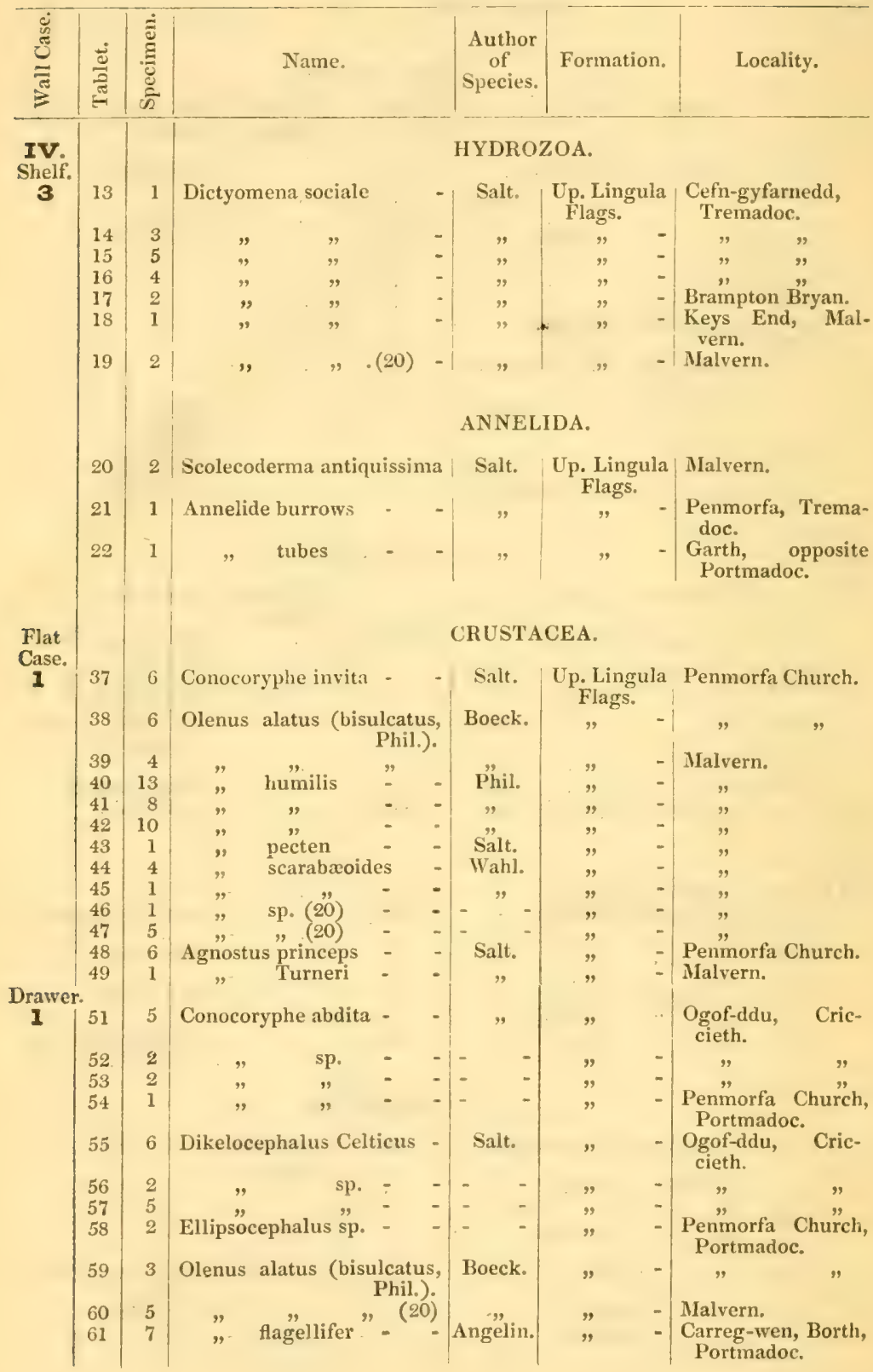




\begin{tabular}{|c|c|c|c|c|c|c|}
\hline 这 & हैं & हूँ & Name. & $\begin{array}{c}\text { Author } \\
\text { of } \\
\text { Species. }\end{array}$ & Formation. & Locality. \\
\hline
\end{tabular}

1

\section{Crustacel-ront.}

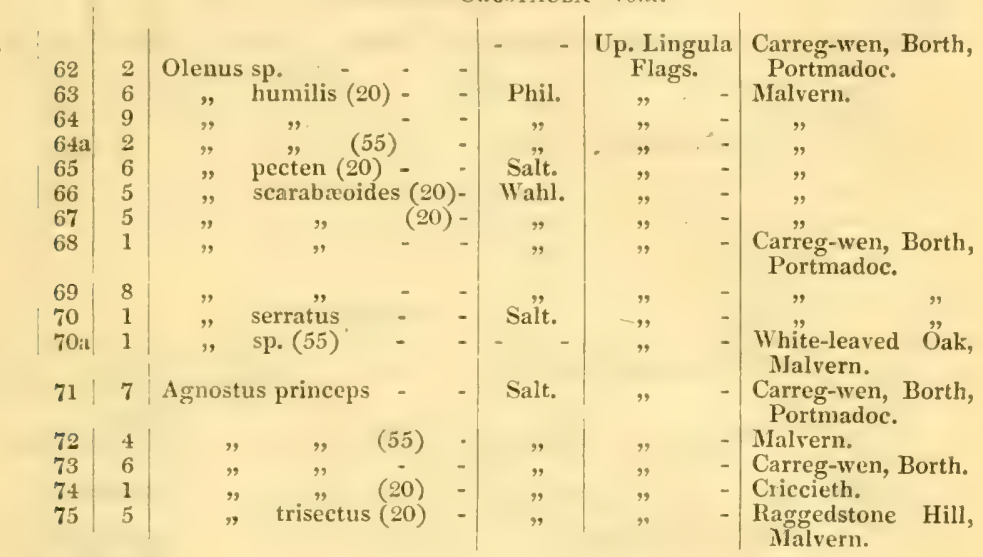

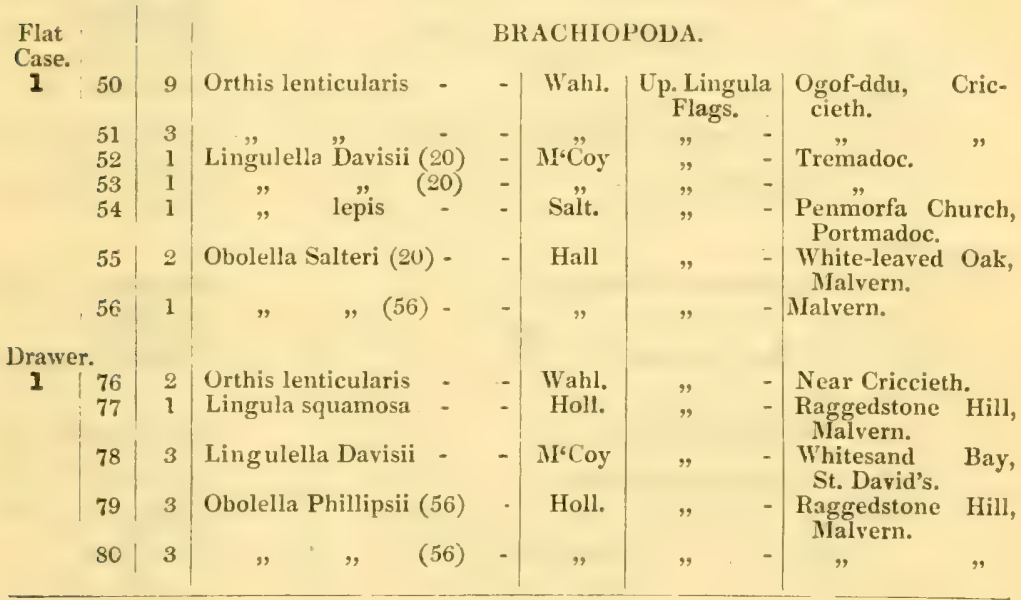

\section{LOWER SILURIAN-cont.}

IOWER TREMADOC.

\begin{tabular}{|c|c|c|c|c|c|c|}
\hline 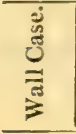 & हैं & ஹू & Name. & $\begin{array}{l}\text { Author } \\
\text { of } \\
\text { Species. }\end{array}$ & Formation. & Locality. \\
\hline $\begin{array}{l}\text { IV. } \\
\text { Shelf. }\end{array}$ & & & \multicolumn{4}{|c|}{ ECHINODERMATA. } \\
\hline 3 & 23 & 1 & $\begin{array}{l}\text { Palaasterina Ramseyensis } \\
\qquad \text { (a cast) (52). }\end{array}$ & Hicks & $\begin{array}{c}\text { Lower } \\
\text { Tremadoc. }\end{array}$ & Ramsey Island. \\
\hline
\end{tabular}




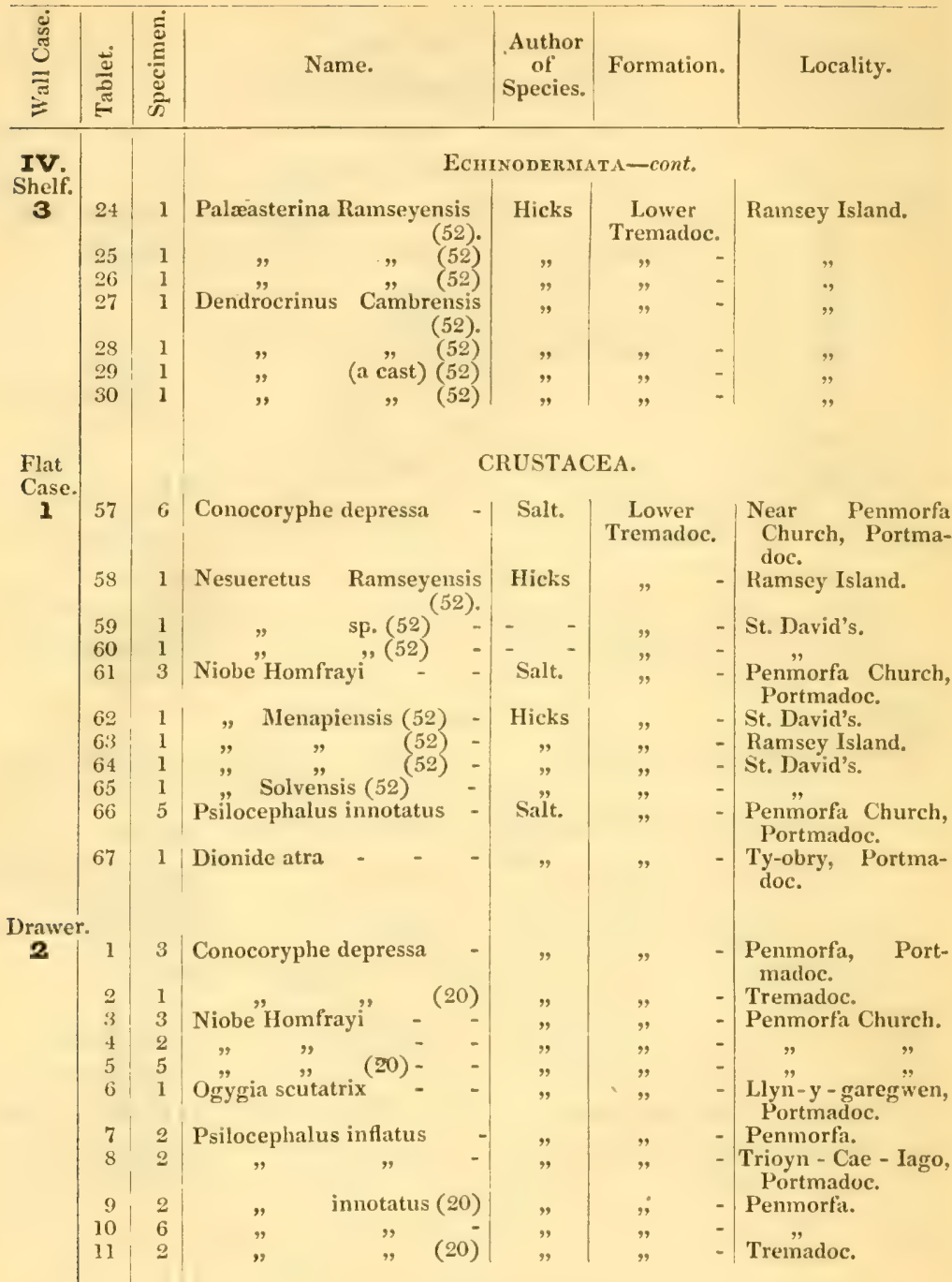

Flat

Case.

\section{BRACHIOPODA.}

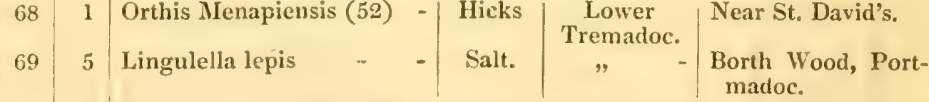

\section{LAMELLIBRA NCHIA'TA.}

\begin{tabular}{l|c|cr|c|c|c}
$71)$ & 1 & Modiolopsis & $\begin{array}{r}\text { Cambrensis } \\
(52)\end{array}$ & Hicks & $\begin{array}{c}\text { Lower } \\
\text { Tremadoc. }\end{array}$ & Ramsey Island. \\
71 & 2 & Homfrayi &, &, & &
\end{tabular} 


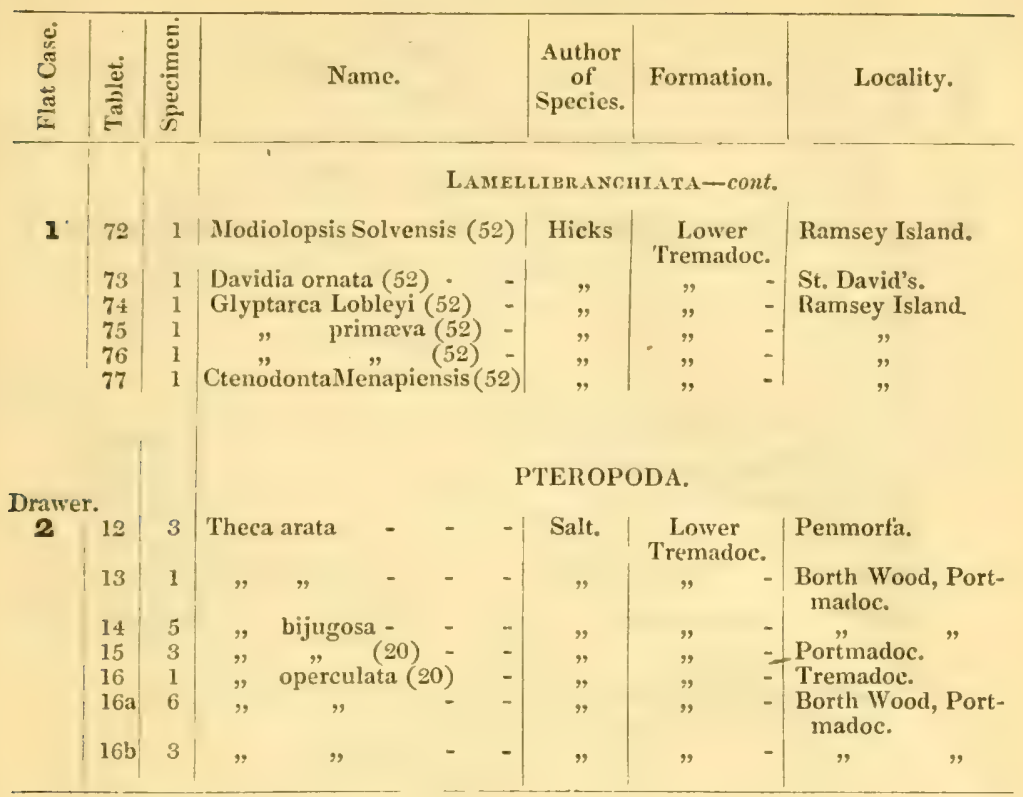

\section{LOWER SILURIAN-cont.}

UPPER TRMMADOC.

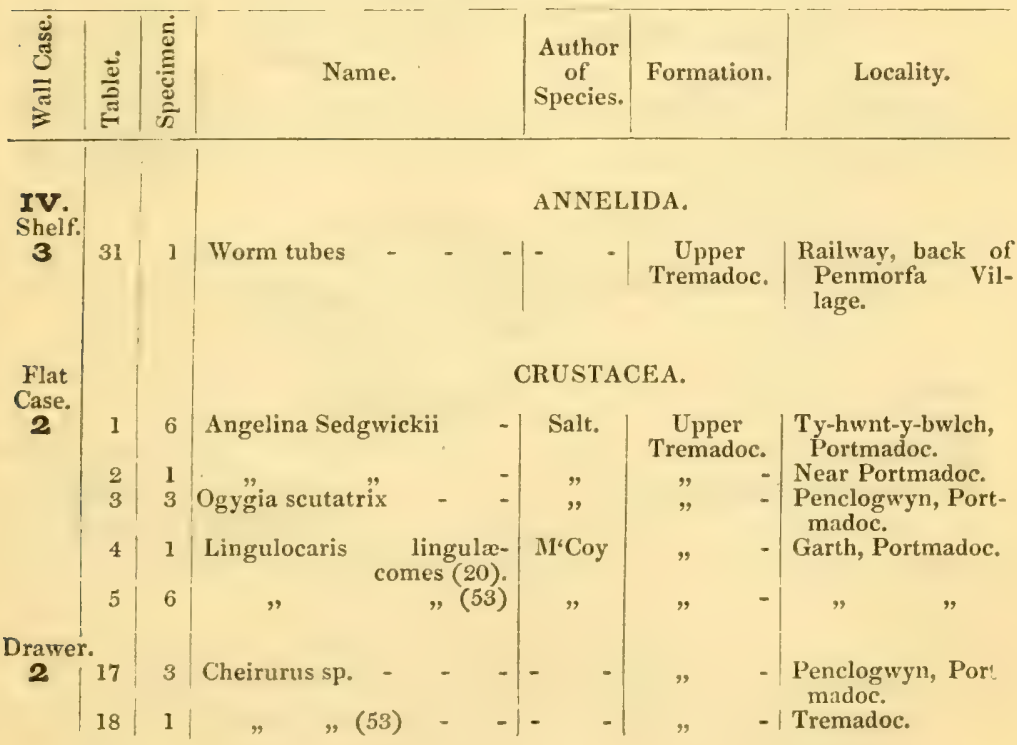




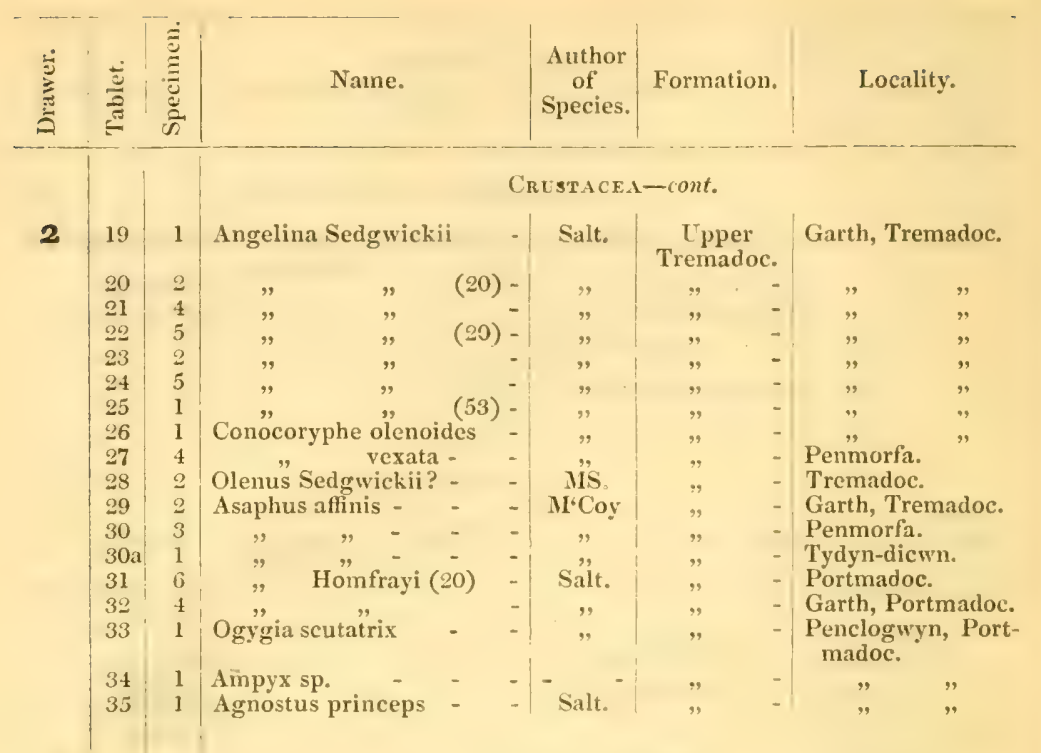

Flat

Case.

2

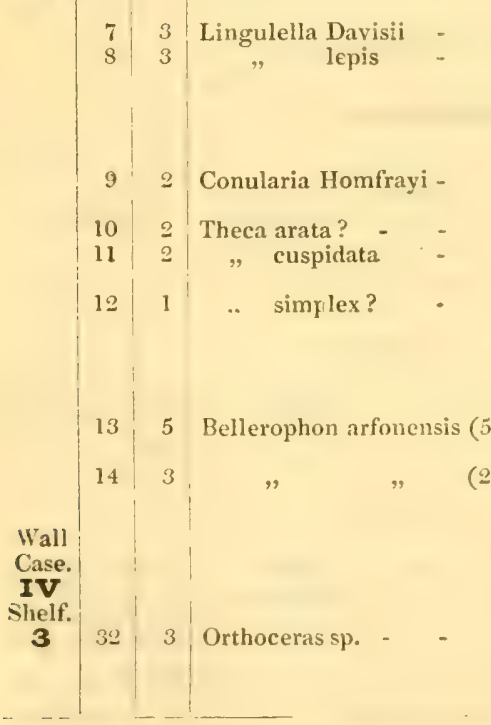

BRACHIOPODA.

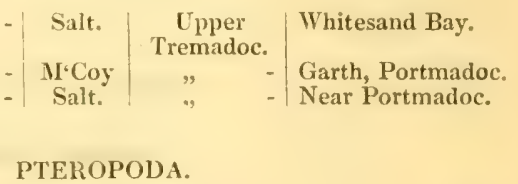

PTEROPODA.

- Salt. Ipper Garth, Portmadoc. Tremadoc.

$$
\begin{aligned}
& \text {, " } \quad \text { - Near Portmadoc. } \\
& \text { " " - - Ty - Wwnt-y-bwlch, } \\
& \text { - Garth, Portmadoc. }
\end{aligned}
$$

\section{HETEROPODA.}

3) Salt Lpper Garth, Portmadoc. Tremadoc.

\section{CEPHALOPODA.}

1. Garth, PortUpper $\left\{\begin{array}{l}\text { madoc. } \\ \text { S. }\end{array}\right.$ Tremadoc. $\left\{\begin{array}{c}\text { 2. Ty - hwnt - y - } \\ \text { bwich. }\end{array}\right.$ 


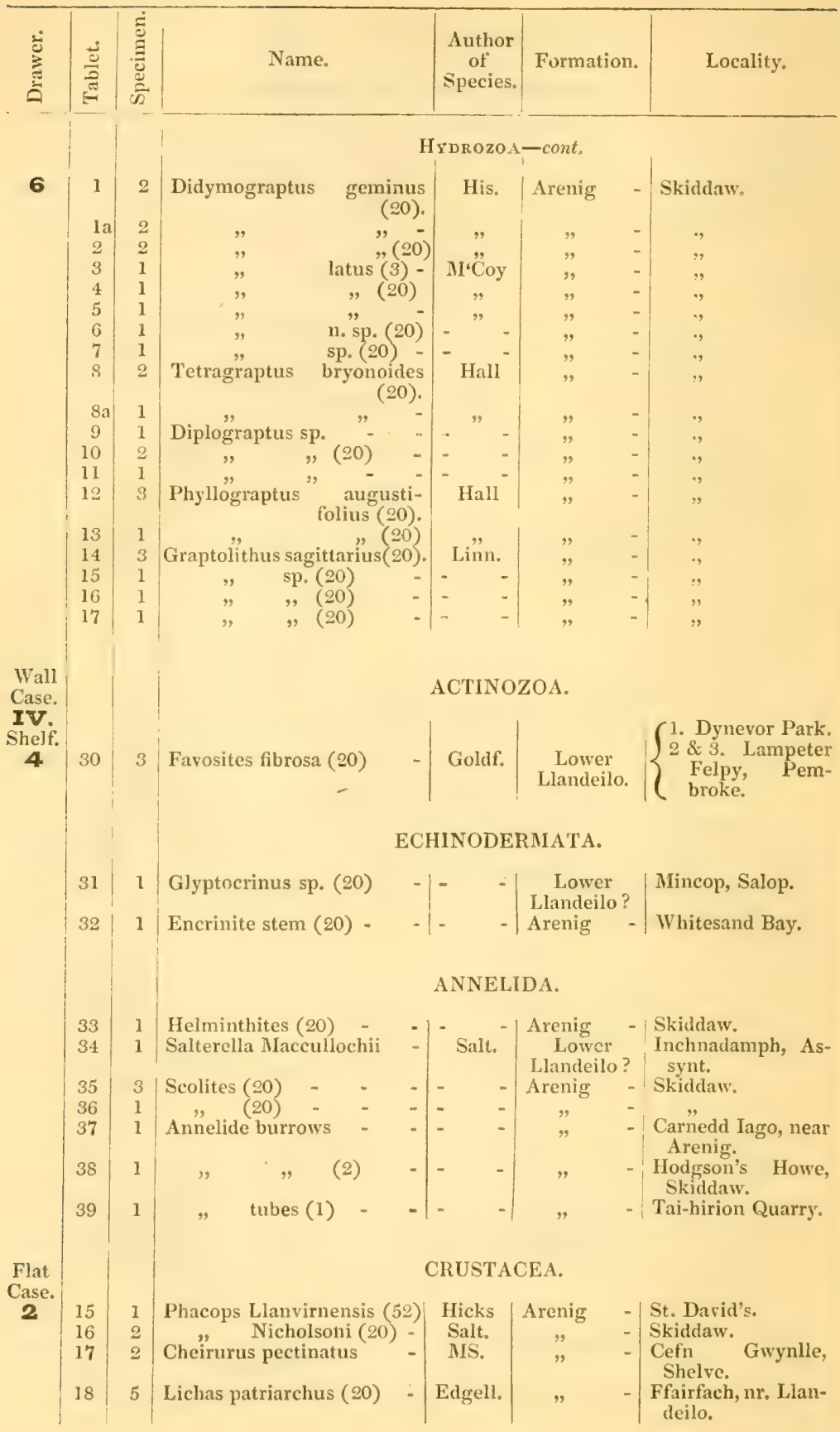




\begin{tabular}{|c|c|c|c|c|c|c|}
\hline 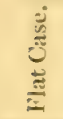 & 岇 & 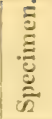 & Name. & $\begin{array}{l}\text { Author } \\
\text { of } \\
\text { Species. }\end{array}$ & Formation. & Locality. \\
\hline
\end{tabular}

\section{Crustices-cont.}

2. 19 | 1 Calymene Blumenbachii, Sait. Lower? Llandeilo.

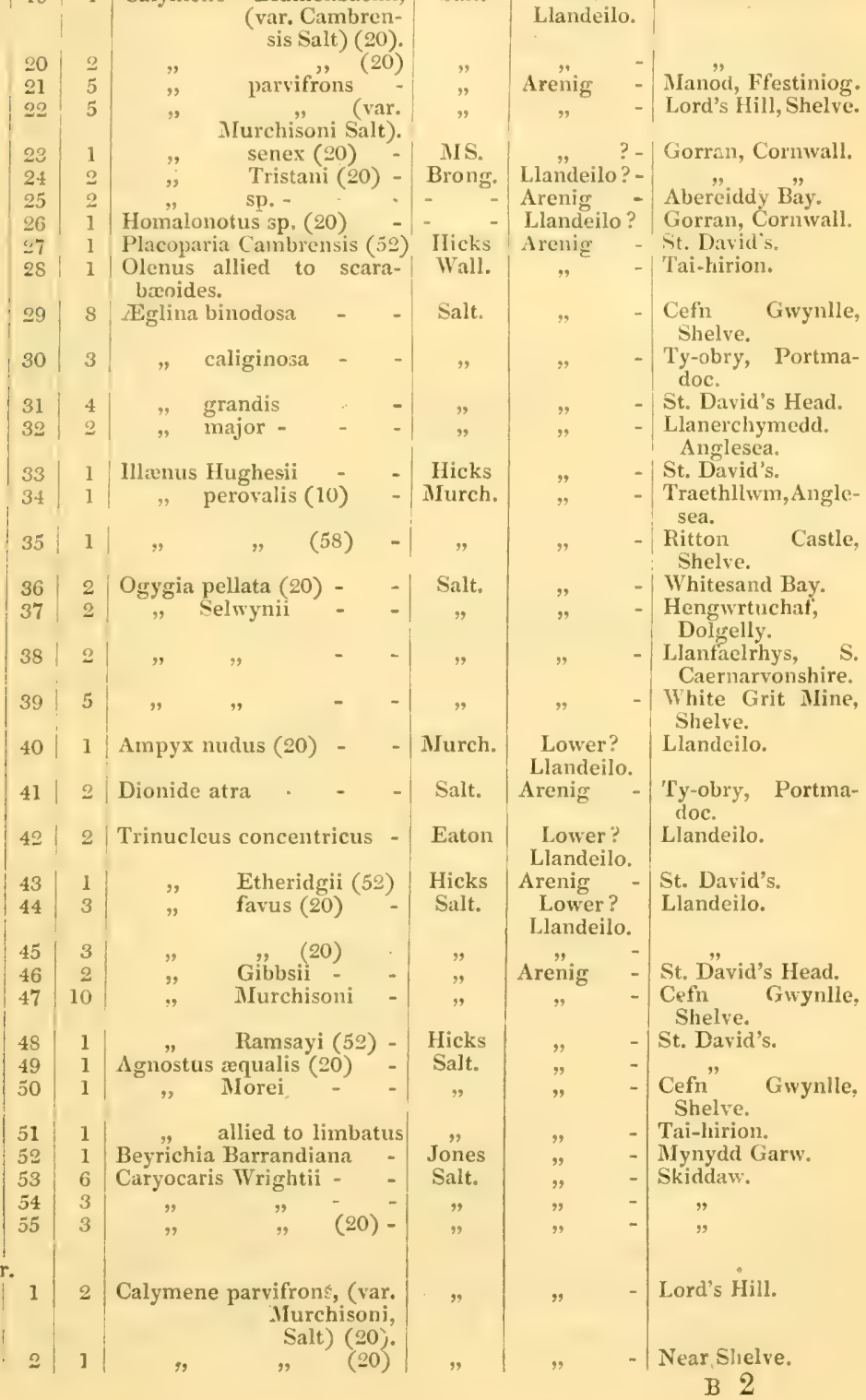




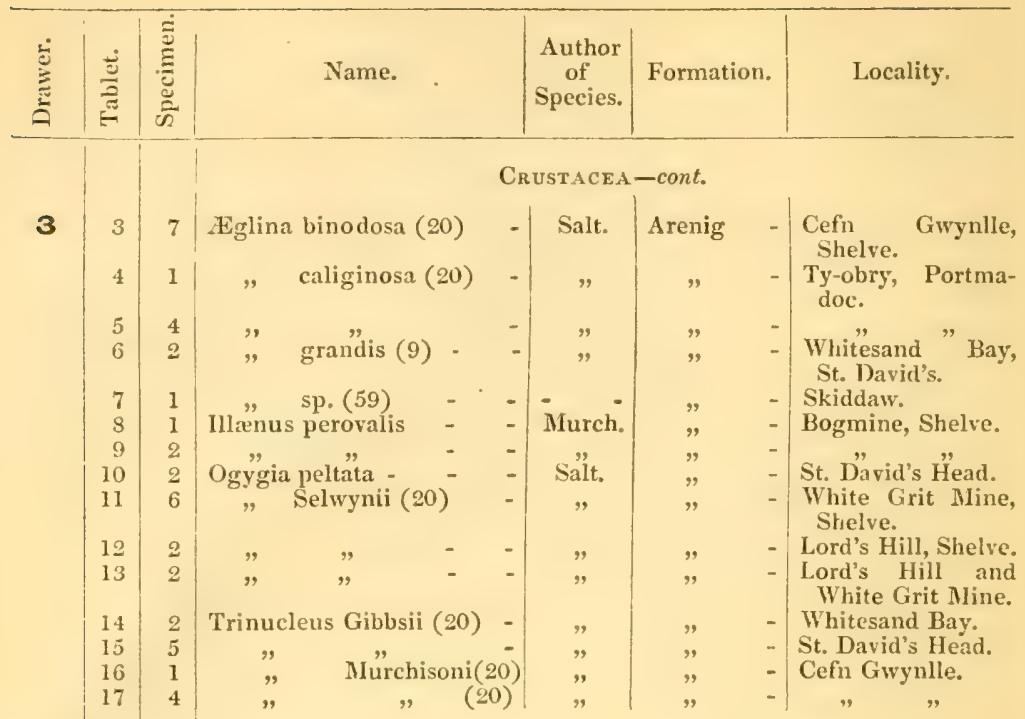

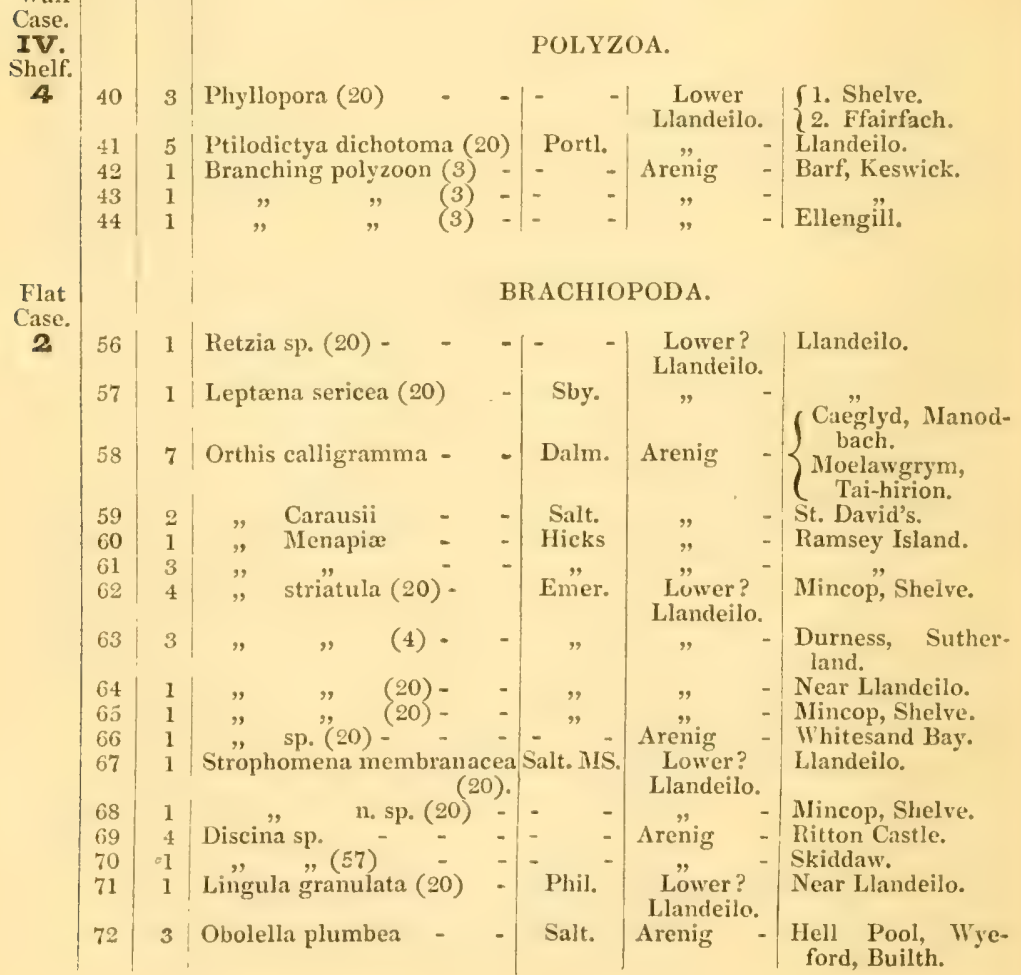

POI,YZOA. 


\begin{tabular}{|c|c|c|c|c|c|c|}
\hline 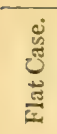 & 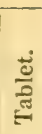 & 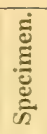 & Name. & $\begin{array}{l}\text { Author } \\
\text { of } \\
\text { Species. }\end{array}$ & Formation. & Locality. \\
\hline
\end{tabular}

\begin{tabular}{|c|c|c|c|c|c|c|c|c|c|c|c|}
\hline \multirow{5}{*}{2} & & & \multicolumn{9}{|c|}{ BRACHIOPODA-cont. } \\
\hline & 73 & 5 & Obole & plum & bea ( & (20) & - & Salt. & Arenig & - & White Grit Mine, \\
\hline & 74 & 4 & $\eta$ & & & .. & -1 & ", & ", & 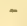 & \\
\hline & 75 & 4 & $"$ & sp. & - & - & - & 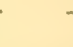 & ", & - & $\begin{array}{l}\text { Ty-obry, Portma- } \\
\text { doc. }\end{array}$ \\
\hline & 76 & 2 & ", & , & - & - & & . & ", & & $\begin{array}{l}\text { Llanfaclrhys, Caer- } \\
\text { narvonshire. }\end{array}$ \\
\hline
\end{tabular}

\section{LAMELLIBRANCHIATA.}

Drawer.

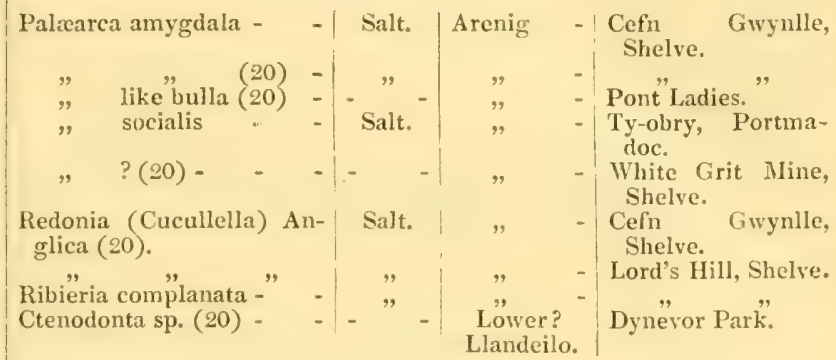

Ctenodonta sp. (20) - $\quad-\quad-\quad-\quad \begin{gathered}\text { Lower? } \\ \text { Llandeilo. }\end{gathered}$ Shelve.

Cefn Gwynlle, Shelve.

Lord's Hill, Shelve.

Dynevor Park".

\section{GASTEROPODA.}

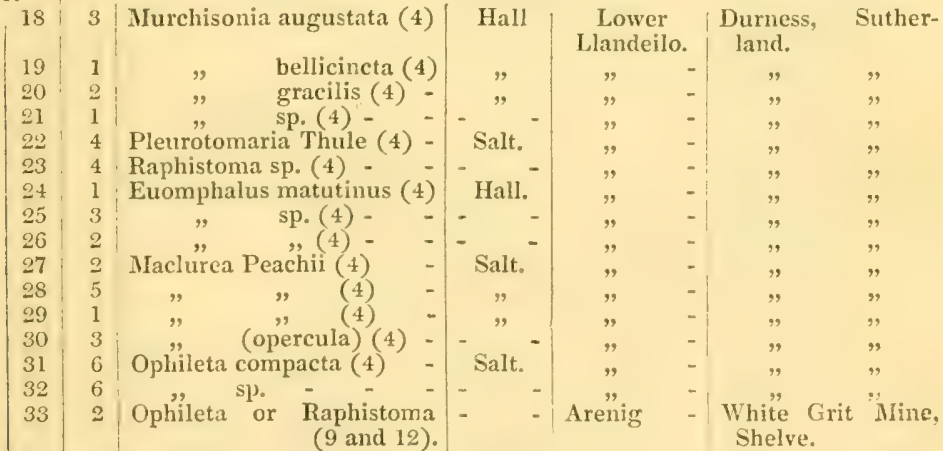

\section{PTEROPODA.}

Conularia corium

- Salt. Arenig

- Ty-obry, Portmadoc. 


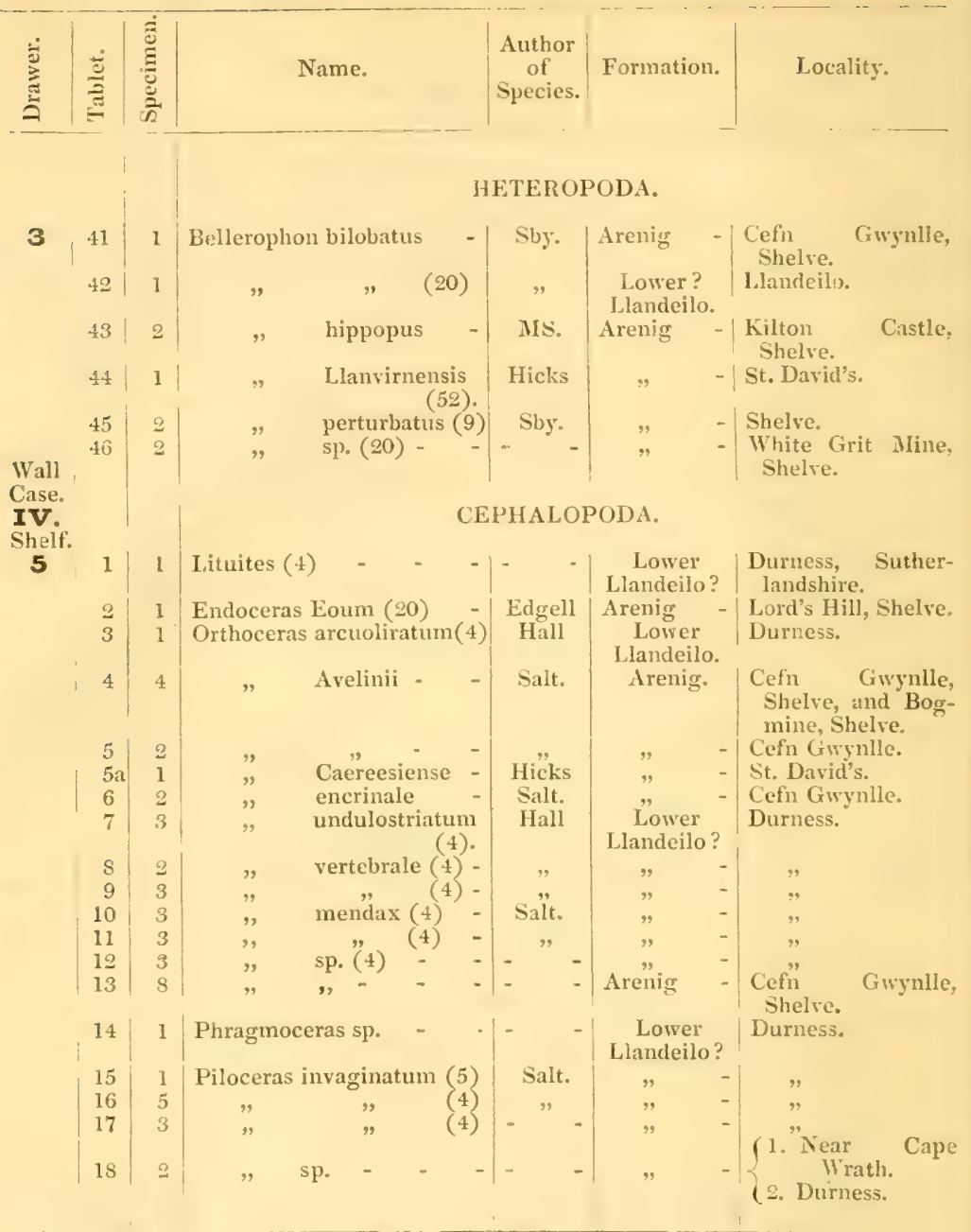

LOWER SILURIAN-cont.

UPPER IIANDEILO.

\begin{tabular}{|c|c|c|c|c|c|c|c|}
\hline 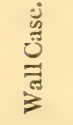 & है & $\begin{array}{l}\text { हैं } \\
\text { हैं } \\
\text { के }\end{array}$ & Name. & & $\begin{array}{c}\text { Author } \\
\text { of } \\
\text { Species. }\end{array}$ & Formation. & Locality. \\
\hline $\begin{array}{l}\text { IV. } \\
\text { Shelf. }\end{array}$ & & & \multicolumn{5}{|c|}{ PLANTE. } \\
\hline 5 & 19 & 6 & Fucoid (6) & -1 & $-\quad-1$ & $\begin{array}{c}\text { Upper } \\
\text { Llandeilo. }\end{array}$ & Wigtownshire. \\
\hline
\end{tabular}




\begin{tabular}{|c|c|c|c|c|c|c|}
\hline 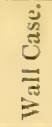 & 苞 & 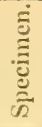 & Name. & $\begin{array}{l}\text { Author } \\
\text { of } \\
\text { Species. }\end{array}$ & Formation. & Locality. \\
\hline
\end{tabular}

IV. Shelf.

5

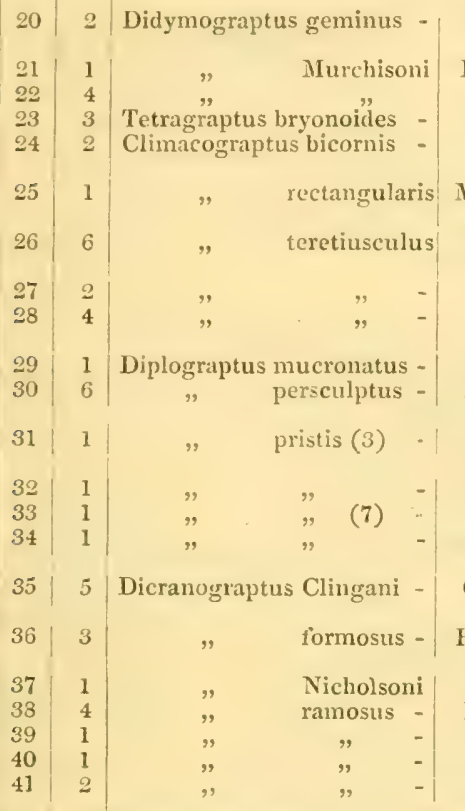

$42 \mid 2$

$43 \quad 1$

\begin{tabular}{l|l|}
43 & 1 \\
44 & 3 \\
\hline
\end{tabular}

Graptolithus Becki

$\begin{array}{ll} & \text { convolutus (8) } \\ \text { Hisingeri }\end{array}$

Hi

$45 \mid 5$

lobiferus (3)-

\begin{tabular}{l|l|}
46 & 1
\end{tabular}

47

| 1

1 2

\begin{tabular}{l|l}
2 & 1
\end{tabular}

\begin{tabular}{l|l}
3 & 1
\end{tabular}

4i 1

5 1

6

7

8

\section{HYDROZOA.}

His. Upper | Bogmine, Shelve.

Llandeilo.

Hall . " $\quad$ - Wexford.

- Clarbeston, N. of Haverfordwest.

- Dobb's Lane, Dumfries.

Cae Mawr, Anglesea.

- Dumfriesshire.

Glenkiln, Dumfries.

Hall

Salt.

His.

Dumfriesshire.

- Gogofau, Caermarthenshire.

- Glenkiln, Dum. fries.

- Hartfell, Dumfries.

- Llangadock.

- Timnagloh, Wexford.

- Castell, 9 miles W. of Builth.

- Near Llanerchymedd, Anglesea.

- Moffat, Dumfries.

Abereiddy Bay.

Dumfriesshire.

Glenkiln, Duinfries.

- Bran Burn, Dumfries.

Moffat, Dumfries.

Bran Burn and Glenkiln, Dumfries.

Bell Craig Burn, Dumfries.

Duff Kinnell, Dumfries.

- Moffat, Dumfries.

Bell Craig Burn, Dumfries.

- Bran Burn, Dum. fries.

- Glenkiln, Dumfries.

- Llanfaelrhys, Caernarvon.

- Moffat, Dumfries.

- Bell Craig Burn, Dumfries.

- Bran Burn, Dum. fries.

Shelve. 


\begin{tabular}{|c|c|c|c|c|c|c|}
\hline 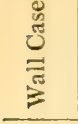 & 总 & 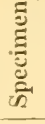 & Name. & $\begin{array}{c}\text { Author } \\
\text { of } \\
\text { Species. }\end{array}$ & Formation. & Locality. \\
\hline $\begin{array}{l}\text { IV. } \\
\text { Sheif. }\end{array}$ & & & \multicolumn{4}{|c|}{ HYDROZOA - cont. } \\
\hline 6 & 9 & 1 & Protovirgularia dichotoma & $\mathrm{M}^{6} \mathrm{Coy}$ & $\begin{array}{l}\text { Upper } \\
\text { Llandeilo. }\end{array}$ & $\begin{array}{l}\text { Glenkiln, } \\
\text { fries. }\end{array}$ \\
\hline Drawe & & & & & & \\
\hline 7 & $\begin{array}{l}1 \\
2\end{array}$ & $\begin{array}{l}1 \\
2\end{array}$ & $\begin{array}{r}\text { Didymograptus Murchisoni } \\
,(20)\end{array}$ & Beck. & $"$ & Builth. \\
\hline & 3 & 3 & $" \quad " \quad "(20)$ & $"$ & $"$ & $"$ \\
\hline & $\begin{array}{l}4 \\
5\end{array}$ & 1 & $" \quad$ sextans? & IHall & $"$ & Hartfoll Dumfries. \\
\hline & 6 & 1 & $\begin{array}{l}\text { Climacograptus teretius- } \\
\text { culus. }\end{array}$ & $\begin{array}{l}\text { Hail } \\
\text { His. }\end{array}$ & $"$ & $\begin{array}{l}\text { Bartfell, Dumiries. } \\
\text { Bran'Burn, Dum- } \\
\text { fries. }\end{array}$ \\
\hline & 7 & 1 & Diplograptus folium (20) - & $"$ & " & $\begin{array}{l}\text { Dobb's Lynn,Dum- } \\
\text { fries. }\end{array}$ \\
\hline & $\begin{array}{l}8 \\
9\end{array}$ & 1 & $" \quad(20)-$ & & $"$ & \\
\hline & $\begin{array}{r}9 \\
10\end{array}$ & 1 & mucronatus - & Hall & $"$ & - Belvoir, Clare. \\
\hline & $\begin{array}{l}10 \\
11\end{array}$ & $\begin{array}{l}2 \\
1\end{array}$ & $\Rightarrow \quad(64)$ & $"$ & $"$ & - Near Llandeilo. \\
\hline & 12 & 1 & $" \quad(64)$ & $"$ & $"$ & Dumfriesshire. \\
\hline & 13 & 1 & pristis (20) - & His. & $"$ & " \\
\hline & 14 & 1 & $" \quad(20) \cdot$ & " & $"$ & $\begin{array}{l}\text { - Dobb's Lynn, Dum- } \\
\text { fries. }\end{array}$ \\
\hline & 15 & 1 & " $\quad(20)$ & " & " & - Hartfell, Dumfries. \\
\hline & 16 & 2 & $" \quad-$ & $"$ & , & - Carneddau Hill, \\
\hline & 17 & 1 & $" \operatorname{var}^{-}(20)$ & $"$ & $"$ & - Belvoir, Clare. \\
\hline & $\begin{array}{l}18 \\
19\end{array}$ & $\begin{array}{l}1 \\
1\end{array}$ & Helicograptus gracilis & Häll & $"$ & - Builth. \\
\hline & 20 & 1 & " " & $"$ & $"$ & - Dumfriesshire. \\
\hline & 21 & 1 & Nemagraptus? (20) & - -1 & ” & - Hartfell, Dumfries. \\
\hline & 22 & 2 & Pleurograptus linearis (20) & Carr. & " & - \\
\hline & 23 & 1 & $\begin{array}{c}\text { Dicellograptus } \begin{array}{c}\text { Forcham* } \\
\text { meri. }\end{array}\end{array}$ & Gein. & " & - Kilmacreagh,Clare. \\
\hline & 24 & 1 & Cyrtograptus hamatus & ${ }^{-}$Baily ${ }^{-}$ & $"$ ? & - Belvoir, Clare. \\
\hline & 25 & 1 & & & & $\begin{array}{l}\text { - Garrangrena, Tip. } \\
\text { perary. }\end{array}$ \\
\hline & 26 & 1 & " & " & $"$ & $\begin{array}{l}- \\
\text { Reafadda, Tippe- } \\
\text { rary. }\end{array}$ \\
\hline & 27 & 1 & Graptolithus Hisingeri (20) & Carr. & " & - Bell Craig Burn, \\
\hline & 28 & 1 & lobiferus (3)- & $\mathrm{M}^{\circ} \mathrm{Coy}$ & " & - Dumfriesshire. \\
\hline & 39 & 1 & $(20)$ & $"$ & $"$ & - Bell Craig Burn, \\
\hline & 30 & 1 & Nilssoni (20) & Barr. & " & - Garpel, Dumfries. \\
\hline & 31 & 1 & sagittarius (3) & Linn. & $"$ & $\begin{array}{l}\text { - Duff' Kinnell, Dum- } \\
\text { fries. }\end{array}$ \\
\hline & 32 & 2 & Sedgwickii(20) & Portl. & " & - Bell Craig Burn, \\
\hline & 33 & 1 & " $\quad(20)$ & , & " & $\begin{array}{l}\text { Dobb's Lynn, Dum- } \\
\text { fries. }\end{array}$ \\
\hline & 34 & 1 & $\because \quad(8)$ & " & " & - Moflat, Dumfries. \\
\hline & $\begin{array}{l}35 \\
36\end{array}$ & $\frac{2}{1}$ & $" \quad(20)$ & $"$ & $"$ & - Bell" Craig "Burn. \\
\hline & & & (ख) & $"$ & " & Dumfries. \\
\hline & $\begin{array}{l}37 \\
38\end{array}$ & 1 & tenuis? (20)- & $"$ & " & - Dumfriesshire. \\
\hline & $\begin{array}{l}38 \\
39\end{array}$ & 1 & $\begin{array}{c}\text { tenuis? } \\
\text { " ? }(20)=\end{array}$ & $"$ & $"$ & - Garpool, Dumfries. \\
\hline & 40 & 1 & $?(20)=$ & - & $"$ & \\
\hline & 41 & 1 & (20) - & -1 & $"$ & - Garpool, Dumfries. \\
\hline & 42 & 1 & $"$ & - & $"$ ? & $\begin{array}{l}\text { - Renfadda, Tippe- } \\
\text { rary. }\end{array}$ \\
\hline
\end{tabular}




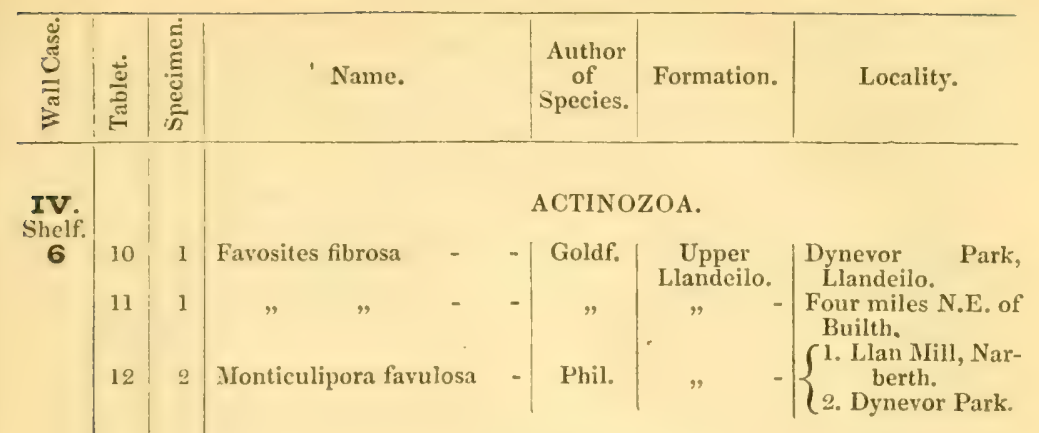

\section{ECHINODERMIATA.}

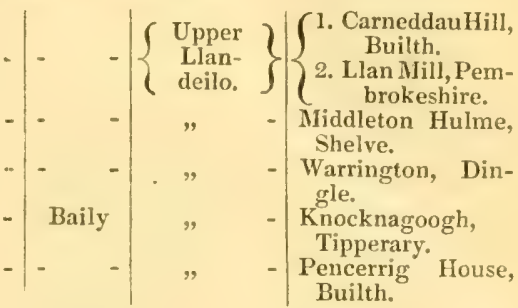

ANNELIDA.

18 I Nereites Cambrensis (5) - $\mid$ Murch. $\mid$ Upper $\mid$ Hunter Heck, Mof-

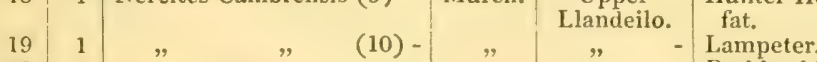

201 " sp. - - - - - " " - Peeblesshire

21 1 Tracks of Nereites (3) _ - " . - Barling, Kirk Tracks of Ne " $\quad$ - $\begin{gathered}\text { Barling, Kir } \\ \text { brightshire. }\end{gathered}$

Palæochorda sp. - - ${ }^{-} \mid$iIc Coy $^{-} \quad, \quad$ Pyritonema fasciculus (20) $\mathrm{II}^{6} \mathrm{Coy} \quad, \quad$ - Near Builth. Nemertites sp. (1i) - - - " - " " $\quad$ - $\quad$ - Thorniclee,

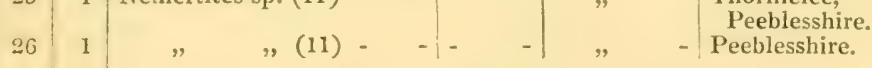

\section{CRUSTACEA.}

\begin{tabular}{|c|c|c|c|c|c|c|c|c|}
\hline 1 & 1 & Phacops H & Iughesii ( & 20) & MS. & Upper & & Ffairfach, nr. Llan- \\
\hline 2 & 1 & Cheirurus & Sedgwic & sii & M'Coy & " & & $\begin{array}{l}\text { Upper Solva, St. } \\
\text { David's. }\end{array}$ \\
\hline 3 & 1 & & & (18) & & " & & Builth. \\
\hline & 3 & Acidaspis & Jamesii & & Salt. & $"$ & - & $\begin{array}{l}\text { Newtown Head, } \\
\text { Waterford. }\end{array}$ \\
\hline 5 & 1 & $"$ & " & - & " & ", & & $\begin{array}{l}\text { Duncannon, Wex- } \\
\text { ford. }\end{array}$ \\
\hline 6 & 1 & " & n. sp. & - & - $\quad-$ & , & - & $\begin{array}{l}\text { Pencerrig House, } \\
\text { Builth. }\end{array}$ \\
\hline 7 & 5 & Calymene & brevicapi & tata - & Portl. & , & - & $\begin{array}{l}\text { Dyneror } \\
\text { Llandeilo. }\end{array}$ \\
\hline & 2 & , & $\begin{array}{r}\text { Blumenb } \\
\text { (var. Ca } \\
\text { sis, } \mathrm{S}\end{array}$ & $\begin{array}{l}\text { achii } \\
\text { mbren- } \\
\text { ltt.) }\end{array}$ & Salt. & " & - & $\begin{array}{l}\text { Llan Mill, Lla } \\
\text { deilo. }\end{array}$ \\
\hline
\end{tabular}




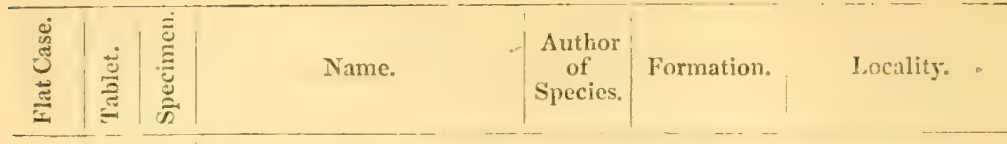

\section{Crustacea-cont.}

Calymene duplicata _. Murch. $\left\{\begin{array}{c}\text { Upper } \\ \text { Llan- }\end{array}\right\}$ Trecoed, Builth. \{ Pencerrig, Builth.

Stoneyford, Haverford west.

- Abereiddy Bay.

Llandeilo.

Golden Grove, Llandeilo.

- Llan "Mill, Narberth.

St. Clare, Haverfordwest.

- Llandeilo.

- Pencerrig, Builth.

Abereiddy Bay.

Wellfield, Builth.

- Newtown, Waterford.

- Builth.

Wellfield, Builth.

- Hope Quarry, Chirbury.

- Llandeilo.

- Buillh.

Ogygia Buchii (60) - - Brong.

$$
\begin{array}{ccc|c}
" \prime & - & - & " \\
" \prime & - & - & " \\
\text { Corndensis ? } & - & \text { Murch. }
\end{array}
$$

,

Pencerrig House, Builth.

Bwlch-cefn, near Llandegley.

Trecoed, Builth.

- Pensarn, near Caermarthen.

Newtown, Waterford.

Carneddau, Builth.

Builth.

Wellfield, Builth.

Garn.

Abereiddy Bay.

Panblewin, Narbeth.

f Naerdy Back. Penllwynan.

- Abereiddy Bay.

- Pencerrig, Builth.

- ?

- Llangadoc.

- Dynevor Park.

- Builth. 


\begin{tabular}{|c|c|c|c|c|c|c|}
\hline 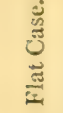 & 兽 & 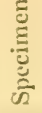 & 'Name. & $\begin{array}{c}\text { Author } \\
\text { of } \\
\text { Species. }\end{array}$ & Formation. & Locality. \\
\hline
\end{tabular}

Crustacea-cont.

3

$$
\begin{array}{l|l|l|r}
55 & 9 & \text { Agnostus M.Coyi } \\
56 & 6 & \text { " } & , 2
\end{array}
$$

Drawer.

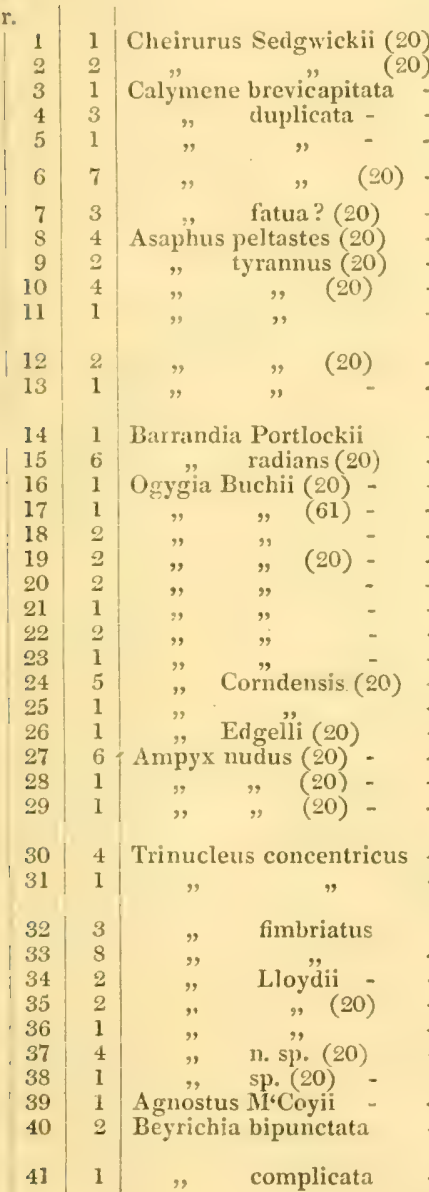

41 1 , complicata

42
43

,

(20)

-

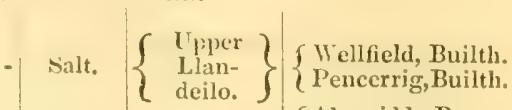
f Abereiddy Bay. \{Pencerrig.

- Pencerrig, Builth. Abereiddy Bay.

- Llandeilo.

- Abereiddy Bay.

- Builth.

$\int$ Abereiddy Bay.

$\{$ Pencerrig.

Ffairfach.

Llandeilo.

Salt.

Murch.

,

9

,

Salt.

$\mathrm{I}^{\circ} \mathrm{Coy}$

Brong.

"

"

"

,

Murch.

Salt. MS.

Murch.

Eaton

Murch.

,

"

,

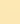

Salt.

MS.

Salt.

Pont"Ladies, Llan. deilo.

Dynevor Park.

Coedcion, Llangadoc.

- Pencerrig, Builth.

- Builth.

- Llandeilo.

- Wellfield, Builth.

- "

- Carneddau, Builth.

- Builth.

- Gilwern, nr. Builth.

- Penrhyn,nr. Builth.

- Whitesand Bay.

- Pencerrig, Builth.

- Abereiddy Bay.

- Pencerrig, near Builtin.

- Cornden Grits.

- Middleton, East of Chirbury.

- Givernyfed, Builtil.

- Pencerrig, Builth.

- Near Builth.

- Pencerrig, Builth.

- Middleton.

Builth.

Hell Pool, Wyeford, Builth.

Middleton, Chirburg.

- Ffairfach

- Llan Mill, Narberth.

- Ciln Park.

- Llan Mill, Nar. berth. 


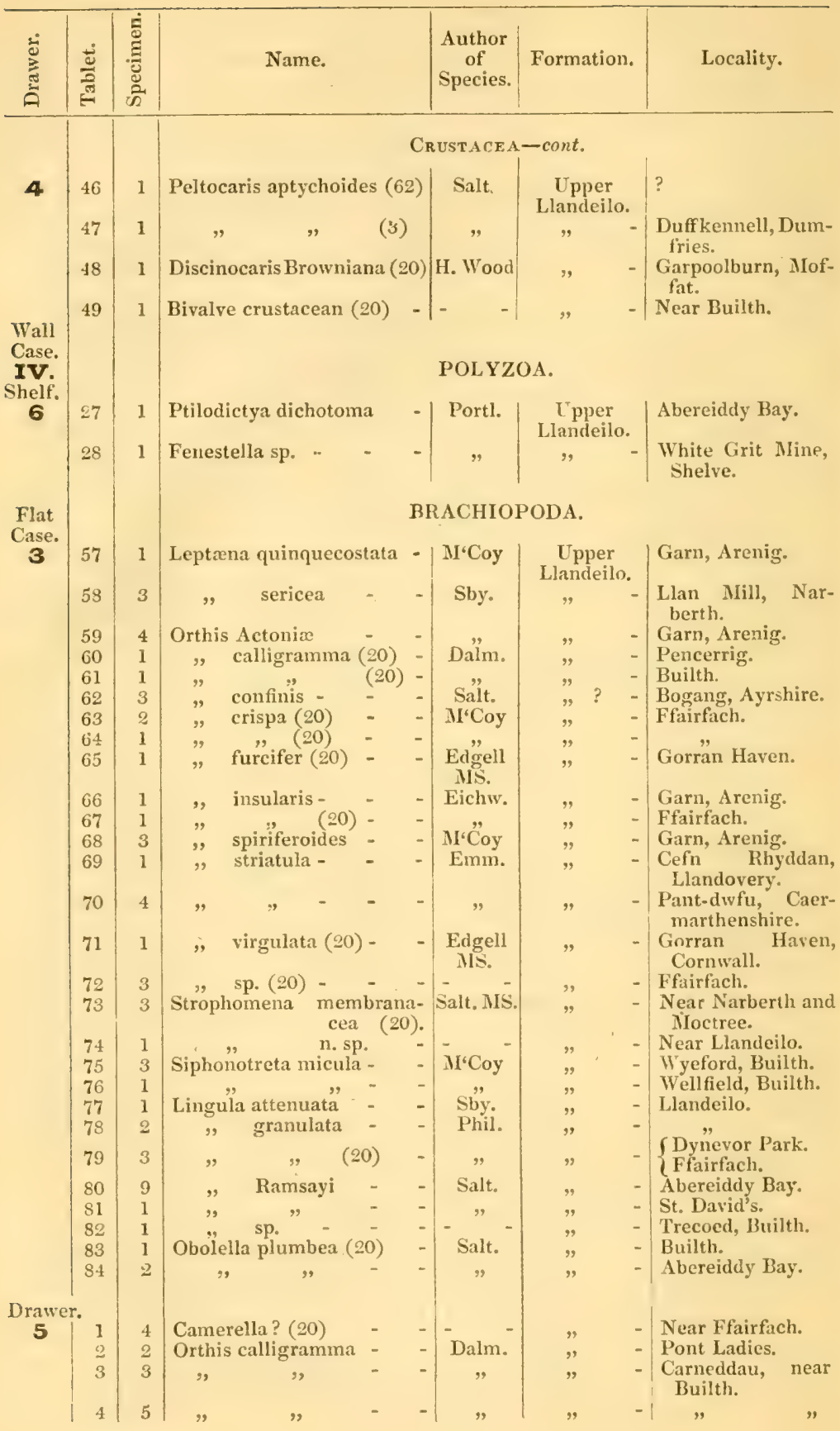




\begin{tabular}{|c|c|c|c|c|c|c|c|c|c|}
\hline 这 & $\frac{\tilde{d}}{\text { हैं }}$ & 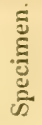 & Name. & & & $\begin{array}{l}\text { Author } \\
\text { of } \\
\text { Species. }\end{array}$ & Formation & & Locality. \\
\hline \multirow{19}{*}{5} & & & \multicolumn{7}{|c|}{ BRACHIOPODA-cont. } \\
\hline & 5 & 2 & Orthis calligramma & - & - & Dalm. & $\begin{array}{c}\text { Upper } \\
\text { Llandeilo }\end{array}$ & & Builth. \\
\hline & 6 & 4 & confinis - & - & - & Salt. & , & - & Bogang, Ayrshire. \\
\hline & 7 & 1 & testudinaria & - & - & Dalm. & , & - & Gorran, Cornwall. \\
\hline & s & 3 & vespertilio & - & - & Sby. & - " & - & Ciln Park, Narberth. \\
\hline & 9 & 2 & "sp. - - & - & - & - & " & - & $\begin{array}{l}\text { Gorran Haven, } \\
\text { Cornwall. }\end{array}$ \\
\hline & 10 & 5 & Strophomena n. sp. & - & - & - & " & - & Llan Mill. \\
\hline & 11 & 3 & , & - & - & - &, & - & , \\
\hline & 12 & 1 & , & - & - & & , & - & Golden Grove. \\
\hline & 13 & 2 & Discina crassa - & - & - & Hall & $"$ & - & Builth. \\
\hline & 14 & 3 & Siphonotreta micula & $(3)$ & - & M'Coy & ", & - & $\begin{array}{l}\text { Glenkiln, Dum- } \\
\text { friesshire. }\end{array}$ \\
\hline & 15 & 7 & " & $(20)$ & - & " & ", & - & Builth. \\
\hline & 16 & 5 & , sp. $(20$ & & - & - & $"$ & - & Llandeilo. \\
\hline & 17 & 1 & Trematis $\mathrm{n} . \mathrm{sp} .(20)$ & - & - & - & $"$ & - & $\begin{array}{l}\text { Ffairfach, Dum- } \\
\text { friesshire. }\end{array}$ \\
\hline & 18 & 3 & Lingula attenuata & - & - & Sby. & $"$ & - & $\begin{array}{l}\text { Middleton, East of } \\
\text { Chirbury. }\end{array}$ \\
\hline & 19 & 1 & $(2$ & & - & " & " & - & Builth. \\
\hline & 20 & 1 & $(2$ & & $=$ & $"$ & $"$ & - & \\
\hline & 21 & 3 & ", & - & - & " & " & - & $\begin{array}{l}\text { Coed Sion, Llan- } \\
\text { gadock. }\end{array}$ \\
\hline & $\begin{array}{l}22 \\
23\end{array}$ & $\begin{array}{l}1 \\
4\end{array}$ & Ramsayi (2) & & - & Salt. & ", & - & Abereiddy Bay. \\
\hline
\end{tabular}

IAMELLIBRA NCHIATA.
\begin{tabular}{ll|l|l|l} 
Modiolopsis filosa (20) & - & MS. & $\begin{array}{c}\text { Upper } \\
\text { Llandeilo. }\end{array}$ & Ffairfach. \\
Genus? & & & & -
\end{tabular}
" - Gorran Haven,
Cornwall.
Ctenodonta varicosa (20) - Salt. , _ - Dynevor Park.

Drawer.

\section{GASTEROPODA.}

\begin{tabular}{|c|c|c|c|c|c|}
\hline 24 & 1 & Murchisonia simplex (20) & $\mathrm{M}^{\prime} \mathrm{Coy}$ & $\begin{array}{l}\text { Upper } \\
\text { Llandeilo. }\end{array}$ & Pencerrig, Builth. \\
\hline & & Cyclonema crebristria & & , & Cornden Grits. \\
\hline & 4 & Helicotoma Anglica (20) & MS. & , & Ffairfach. \\
\hline & 1 & Loxonema - & & . & Builth Bridge. \\
\hline
\end{tabular}

\section{PTEROPODA.}

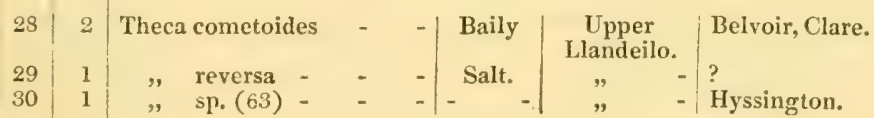

\section{HETEROPODA.}

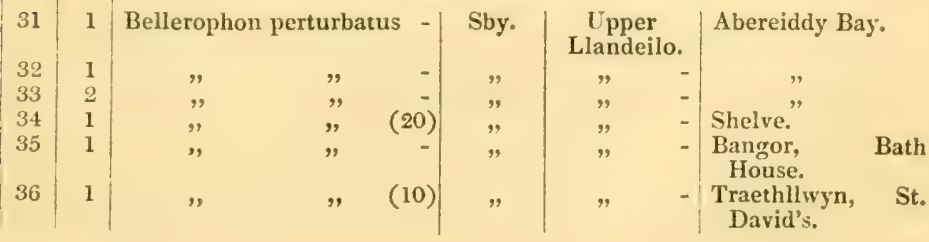


LOWER SILURIAN-cont.

CARADOC.

\begin{tabular}{|c|c|c|c|c|c|c|}
\hline 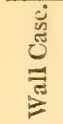 & है & 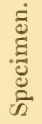 & Name. & $\begin{array}{l}\text { Author } \\
\text { of } \\
\text { Species. }\end{array}$ & Formation. & Locality. \\
\hline
\end{tabular}

$\checkmark$.

Shelf.

1

VARIOUS LARGE SPECIMENS.

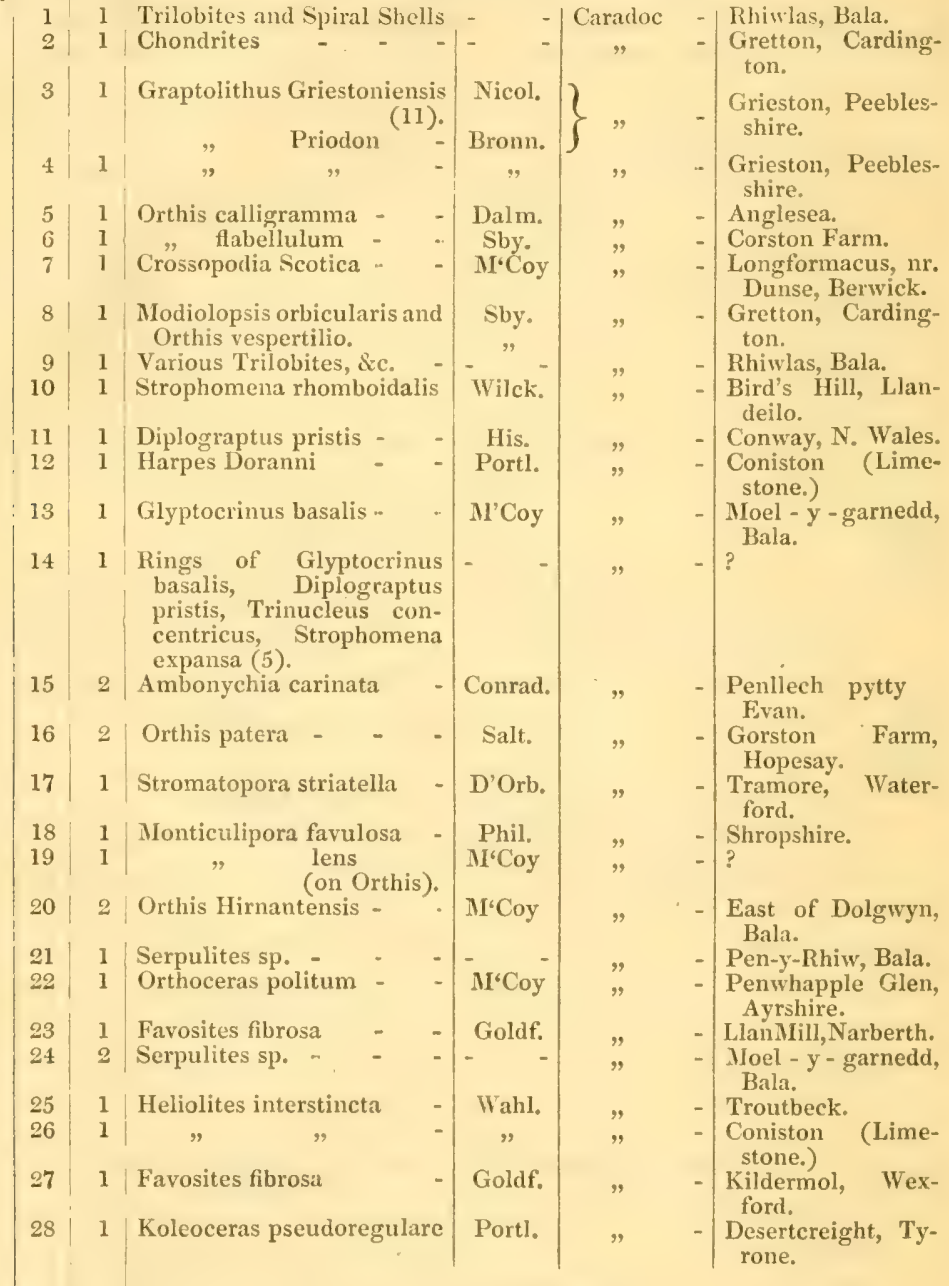

PLANTA.

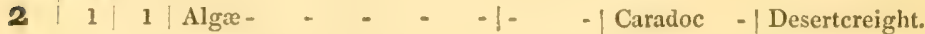




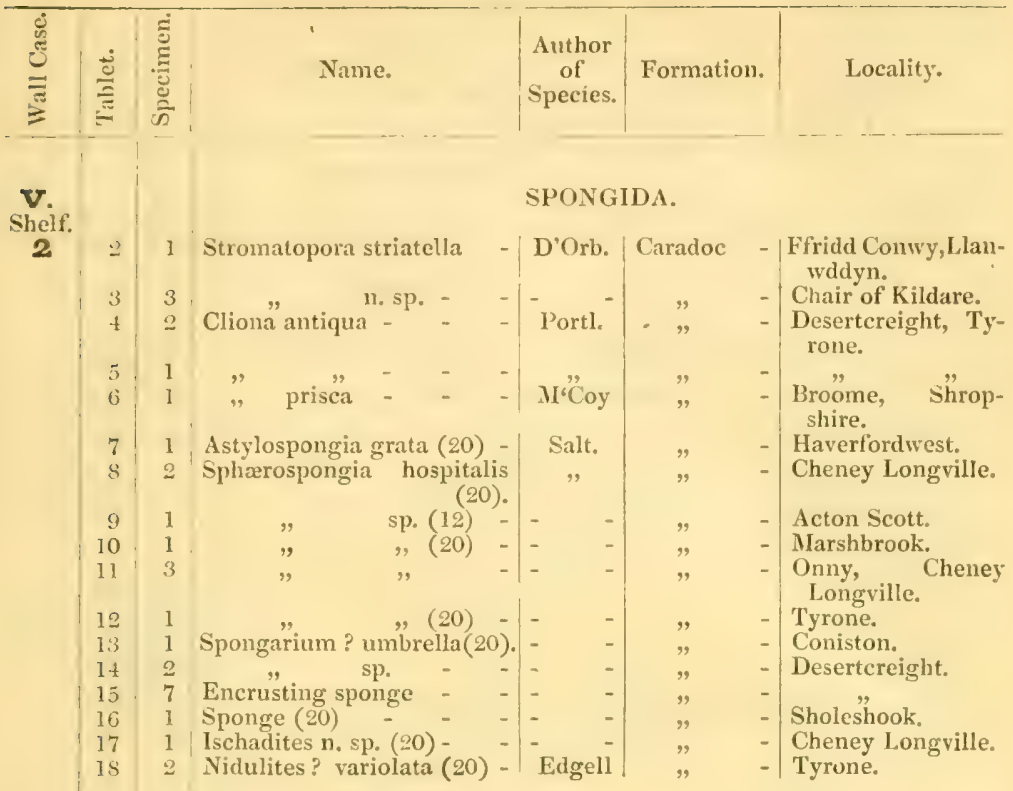

HYDROZOA.

Climacograptus bullatus -

$$
\text { ", }
$$$$
\text { ," }
$$

Diplograptus folium -

$$
\text { , }
$$$$
\text { ", (13) }
$$

pristis (13) -$$
\text { , }
$$$$
\text { , }
$$$$
\text { pristis }
$$

Dicranograptus ramosus

\section{(}

Dendrograptus lentus Graptolithus Hisingeri

", priodon

\begin{tabular}{|c|c|}
\hline Salt. & Caradoc \\
\hline$"$ & $"$ \\
\hline His. & „ \\
\hline$"$ & $"$ \\
\hline " & $"$ \\
\hline$"$ & " \\
\hline " & " \\
\hline$"$ & $"$ \\
\hline$"$ & $"$ \\
\hline$"$ & $"$ \\
\hline " & $"$ \\
\hline " & " \\
\hline " & $"$ \\
\hline$"$ & $"$ \\
\hline Hall & ”, \\
\hline & $"$ \\
\hline Carr. & $"$ \\
\hline$"$ & $"$ \\
\hline Bronn. & $"$ \\
\hline Portl. & $"$ \\
\hline & $"$ \\
\hline & " \\
\hline
\end{tabular}

- Ardwell, Girvan.

- Piedmont "Glen, Ayrshire.

- Fermanagh.

Conway, N. Wales.

- Railway cutting. Conway.

Fermanagh.

Hope Bowdler, Church Stretton.

- Laggan Burn, near Girvan.

- Piedmont Glen, Girvan.

- Robeston Wathen.

Saugh Hill, Girvan.

Soudley.

Little Litterpin, Ayrshire.

- Fermanagh.

- "

Penwhapple Glen, Ayrshire.

N. Wales.

Desertcreight.

- Tyrone.

- Desertcreight. 


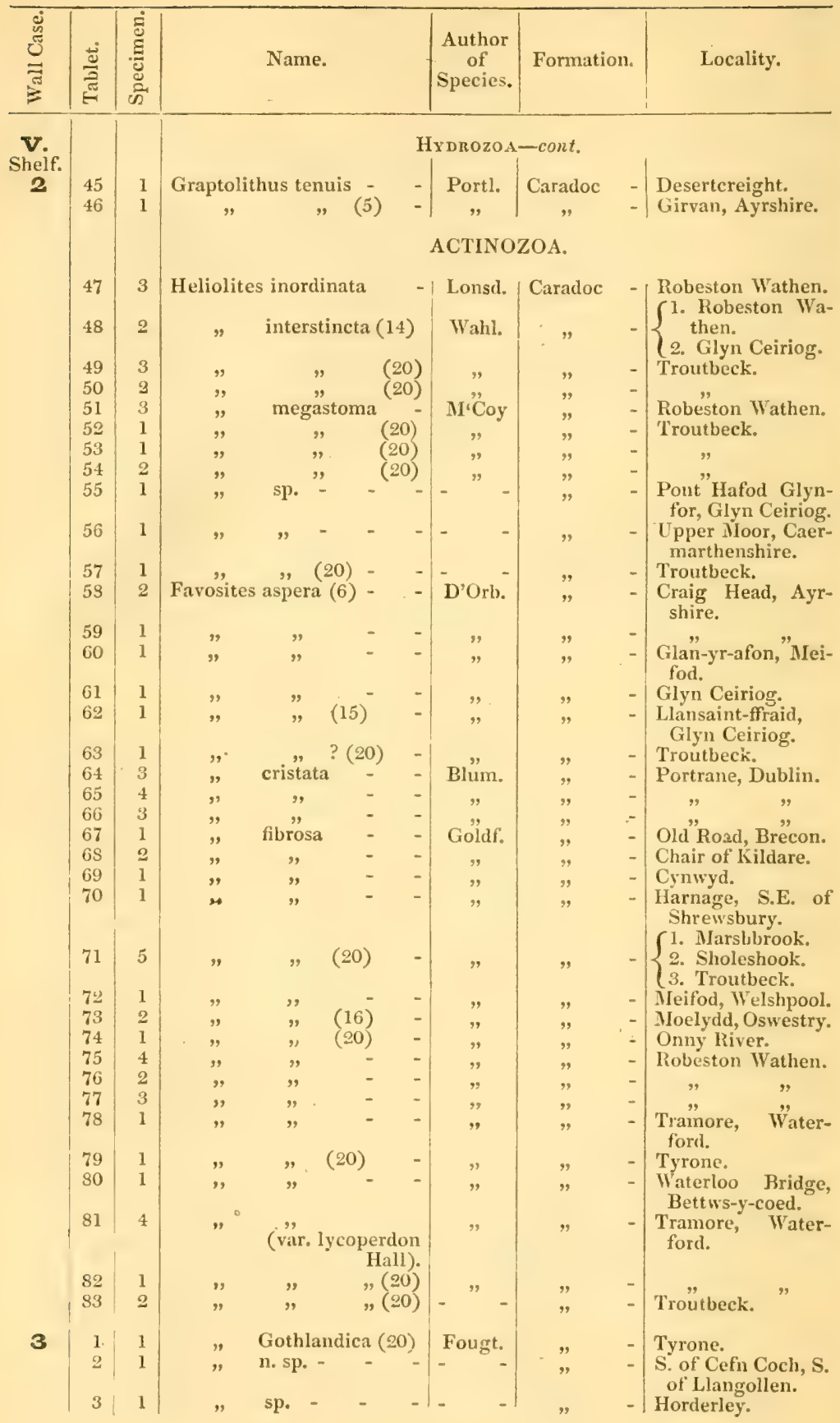




\begin{tabular}{|c|c|c|c|c|c|c|}
\hline 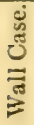 & $\frac{\text { एँ }}{\text { है }}$ & 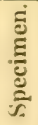 & Name. & $\begin{array}{l}\text { Author } \\
\text { of } \\
\text { Species. }\end{array}$ & Formation. & Locality. \\
\hline
\end{tabular}

\section{v.}

Shelf.

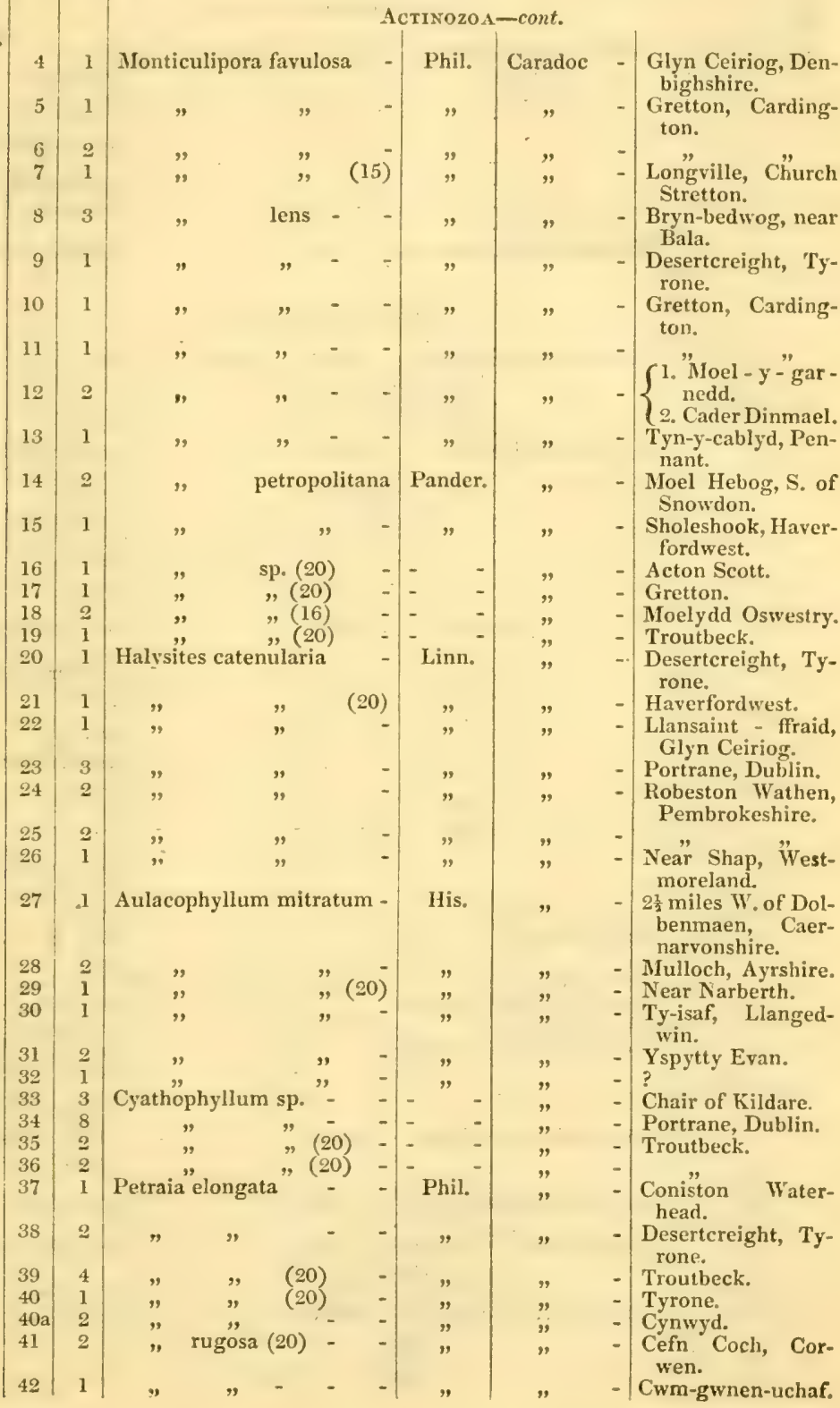

c 


\begin{tabular}{|c|c|c|c|c|c|c|c|}
\hline 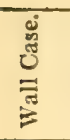 & 岇 & 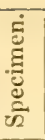 & Name. & $\begin{array}{c}\text { Author } \\
\text { of } \\
\text { Species. }\end{array}$ & \multicolumn{2}{|c|}{ Formation. } & Locality. \\
\hline \multirow{45}{*}{$\underset{\mathbf{3}}{\mathbf{V}}$} & & & \multicolumn{5}{|c|}{ AcTINOZOA-cont. } \\
\hline & $42 a$ & 1 & Petraia sub-duplicata & $\mathrm{M}^{\circ} \mathrm{Coy}$ & Caradoc & & Cynwyd. \\
\hline & 43 & 1 & , n. sp. $(20)$ & & , & & Troutbeck. \\
\hline & 44 & 1 & $\Rightarrow \quad$ sp. $(20)-$ & - & $"$ & - & Helm Gill. \\
\hline & 45 & 3. & $" \quad,-$ & - & $"$ & & $\begin{array}{l}\text { Hendre-wen, Cer- } \\
\text { rig-y-druidion. }\end{array}$ \\
\hline & 46 & 2 & $" \quad "-$ & - & " & 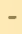 & $"$ \\
\hline & 47 & 4 & $" \Rightarrow(20)=$ & - & $"$ & & Robeston Wathen. \\
\hline & 48 & 1 & $" \quad "(20)=$ & & " & - & Troutbeck. \\
\hline & $\begin{array}{l}49 \\
50\end{array}$ & $\begin{array}{l}2 \\
3\end{array}$ & Syringophyllum organum & 'Linn. & ", & - & Portrane, Dublin. \\
\hline & & & \multicolumn{5}{|c|}{ ECHINODERMATA. } \\
\hline & 51 & . & $\begin{array}{r}\text { Cyathocrinus quinquangu- } \\
\text { laris }(20)\end{array}$ & Phil. & Caradoc & - & Marshbrook. \\
\hline & 52 & 2 & " " ? (20) & $"$ & " & - & Narberth. \\
\hline & 53 & 1 & Glyptocrinus basalis - & $\mathrm{N}^{-} \mathrm{Coy}-$ & $"$ & - & Gretton. \\
\hline & 54 & 1 & $\begin{array}{c}\text { Glyptocrinus basalis - } \\
\#, \quad \text { - }\end{array}$ & $\begin{array}{l}\mathrm{M}^{\circ} \mathrm{Coy} \\
.,\end{array}$ & " & & Guilsfield, Meifod. \\
\hline & 55 & 1 & $" \cdot-$ & $"$ & " & & $\begin{array}{l}\text { Gwrelddian, Llan- } \\
\text { wddyn. }\end{array}$ \\
\hline & 56 & 3 & $(20)$ - & $"$ & " & - & \\
\hline & 57 & 6 & $"$ & $"$ & " & & $\begin{array}{l}\text { Moel - y - garnedd, } \\
\text { Bala. }\end{array}$ \\
\hline & 58 & 2 & $"$ & , & " & - & $\begin{array}{l}\text { Pen-y-rhiw, W. of } \\
\text { Bala. }\end{array}$ \\
\hline & 59 & 1 & lavis - & Portl. & ", & - & $\begin{array}{l}\text { Bardahessiagh, Ty- } \\
\text { rone. }\end{array}$ \\
\hline & 60 & 1 & $" \quad-$ & " & " & - & Desertcreight. \\
\hline & 61 & 2 & " (20) & " & $"$ & - & Tyrone. \\
\hline & 62 & 2 & n. sp. - & " & $"$ & - & $"$ \\
\hline & 63 & 1. & sp. & - & $"$ & - & Bryn Cut. \\
\hline & 64 & 1 & $"$ & - & " & & $\begin{array}{l}\text { Desertcreight, Ty- } \\
\text { rone. }\end{array}$ \\
\hline & 65 & 1 & $\because \quad(20)$ & - & , & - & Gretton. \\
\hline & 66 & 1 & $\Rightarrow \quad(20)$ & - & $"$ & & " \\
\hline & 67 & 4 & $"$ & - & $"$ & - & $\begin{array}{l}\text { Hope Bowdler, } \\
\text { Church Stretton. }\end{array}$ \\
\hline & 68 & 1 & 2801 & - & " & - & Sholeshook. \\
\hline & 69 & 2 & , ? (20) - & - & $"$ & & Gretton. \\
\hline & 70 & 4 & Heterocrinus? - & - & " & - & $\begin{array}{l}\text { N.W. of Welsh- } \\
\text { pool. }\end{array}$ \\
\hline & 71 & 1. & Tetracrinus? (20) - & - & $"$ & - & Troutbeck. \\
\hline & 72 & 2 & Crinoid (auxiliary arin of) & - & " & - & Desertcreigh \\
\hline & 73 & 2 & Crinoidal stem - - & - & $"$ & & Sholeshook. \\
\hline & 74 & 7 & Encrinite stems ( 4 species) & - & , & & Chair of Kildare. \\
\hline & 75 & 1 & ."- & - & " & - & Cheney Longville. \\
\hline & 76 & 2 & $"=$ & - & $"$ & & $\begin{array}{l}\text { Hope Bowdler, } \\
\text { Church Stretton. }\end{array}$ \\
\hline & 77 & 3 & Agelacrinites Buchianus & Forbes & " & $\begin{array}{lll}- & \end{array}$ & $\begin{array}{l}2 \mathrm{~m} \text {. W. of Yspytty } \\
\text { Evan. }\end{array}$ \\
\hline & 78 & 5 & & & $"$ & & \\
\hline & 79 & 9 & Atelocystites oblongus (20) & MS. & $"$ & - & Sholeshook. \\
\hline & 80 & 2 & "n. sp. $(20)$ & ${ }^{-}$us. & $"$ & - & Cheney Longville. \\
\hline & 81 & 1 & $\begin{array}{l}\text { Cyclocystoides Caractaci } \\
\text { (20) }\end{array}$ & Mis. & & & Cheney Longville. \\
\hline & 82 & 1 & , Marstoni & Salt. & " & - & Acton Scott. \\
\hline & 83 & 6 & $\begin{array}{c}\text { Echinospharites arachnoi- } \\
\text { deus }\end{array}$ & Forbes & $"$ & - & Sholeshook. \\
\hline & 84 & 1 & $"(20$ & , & " & - & , \\
\hline & 85 & 1 & $"(2$ & $"$ & $"$ & - & $"$ \\
\hline
\end{tabular}




\begin{tabular}{|c|c|c|c|c|c|c|}
\hline 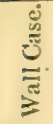 & 冚 & 离 & Name. & $\begin{array}{c}\text { Author } \\
\text { of } \\
\text { Species. }\end{array}$ & Formation. & Locality. \\
\hline
\end{tabular}

\section{v.}

Shelf.

\section{ECHINODERTIATA-continued.}

8611 Echinospharites Balthicus Eichw. , (20) ,

Cara

Bwlch, Cynwyd.

$86 a \mid 1$

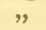

"(20)

\begin{tabular}{l|l|l}
87 & 1 & \\
88 & 4 & 9
\end{tabular}

$89 \quad 6$

$90 \quad 1$

$90 a \quad 1$

$91 \quad 1$

92. 1

\begin{tabular}{|l|l|}
93 & 3
\end{tabular}

$94 \quad 11$

$95 \quad 8$

96.2

\begin{tabular}{l|l}
97 & 3
\end{tabular}

98 | 3

$99 \mid 1$

$100|2|$

4

\begin{tabular}{|c|c|c|c|c|c|}
\hline 1 & 1 & Hemicosmite & es oblongus & - & Pand. \\
\hline la & 1 & $"$. & $\Rightarrow \quad(6$ & 9) & \\
\hline 2 & 3 & " & rugatus & - & Forbes \\
\hline 3 & 2 & " & ", & - & " \\
\hline 4 & 1 & $"$ & , & - & " \\
\hline 5 & 5 & $"$ & " & ? & ", \\
\hline 6 & 2 & " & 9" & - & " \\
\hline 7 & 6 & , & ; & - & ", ( - r - \\
\hline s & 2 & " & squamosus & -1 & " \\
\hline 9 & 1 & 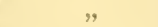 & sp. $\quad-$ & - & - \\
\hline 10 & 5 & " & (allied to) & - & - \\
\hline 11 & 1 & & $?-$ & -. & - \\
\hline 12 & 2 & Pleurocystite & es Rugeri & - & Salt. \\
\hline 13 & 2 & " & n. sp. (20) & - & - \\
\hline 14 & 1 &, & sp. - & - & - \\
\hline 15 & 10 & $"$ & - & - & - \\
\hline 16 & 40 & $"$ & $" \quad-$ & - & - \\
\hline 17 & 6 & Sphæronites & Litchi - & - & Forbes \\
\hline 18 & 3 & " & munitus & - & " \\
\hline 19 & 6 & " & " & - & ", \\
\hline 20 & 4 & " & $"$ & - & , \\
\hline 21 & 6 & $"$ & punctatus & -1 & , \\
\hline 22 & 8 & " & $\Rightarrow \quad(20$ & ) & , \\
\hline 23 & 4 & " & pyriformis & - & \\
\hline 24 & 2 & , & regulatus & - & M.S. \\
\hline 25 & 1 & " & " & $\sim$ & - \\
\hline & 5 & " & stelluliferus & - & Salt. \\
\hline
\end{tabular}

- Coldwell, Westmoreland.

- Gretton.

- Sholeshook.

Troutbeck.

- Cynwyd, near Corwen.

- Cynwyd.

- Pont Hafod Gynfor, Glyn Ceir iog.

- Coniston.

- Sholeshook.

- Rhiwlas, Bala.

- Carrickadaggon, Wexford.

- " "

Troutbeck.

- Sholeshook.

- Near Corwen.

- Great Cresswell, St. Clare.

- Llandewi, Felfrew.

Llanfyllin, Montgomeryshire.

- Newtown, Waterford, and Moyne, Wexford.

-Rhiwlas, Bala.

Sholeshook.

- N. of Bala and near Llanfyllin.

Borwick Fell.

Hendre-wen, Cerrig-y-druidion.

Cynwyd.

- Blaen-y-cwm, Llangadock.

Haverfordwest.

- Bardahessiagh, Tyrone.

- Cefn-Llwydlo, Llandovery.

- Gorwyllt, Llan. rhaidr.

- Sholeshook.

- Rhiwlas, Bala.

- Sholeshook.

Sholeshook.

Rhiwlas, Bala.

Sholeshook.
- Sholeshook.

Rhiwlas, Bala. 


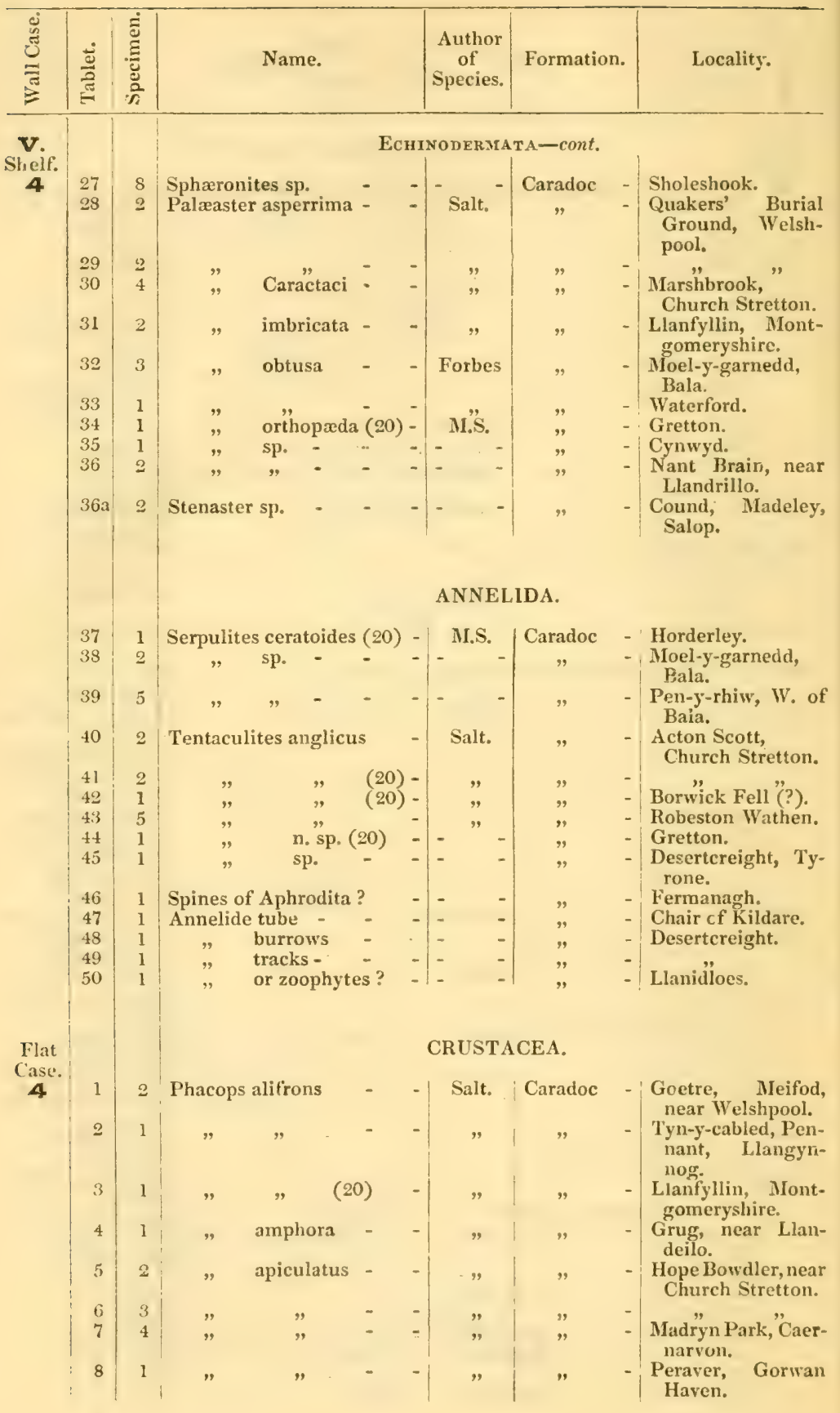




\begin{tabular}{|c|c|c|c|c|c|c|}
\hline 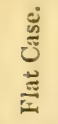 & $\frac{\dot{0}}{\frac{0}{0}}$ & 苋 & Name. & $\begin{array}{c}\text { Author } \\
\text { of } \\
\text { Species. }\end{array}$ & Formation. & Locality. \\
\hline
\end{tabular}

$4 \int_{3}$ Crustacea-cont

\begin{tabular}{|c|c|c|c|c|c|c|c|c|}
\hline 9 & 3 & Phacops & s apiculatus & . & Salt. & Caradoc & - & $\begin{array}{l}\text { Hendre-wen, Cer- } \\
\text { rig-y-druidion. }\end{array}$ \\
\hline 10 & 1 & , & , & (20) - & , & $"$ & -1 & Onny River. \\
\hline 11 & 8 & ," & $"$ & (20) - & $"$ & $"$ & - & Horderley, Salop. \\
\hline 12 & 1 & " & $"$ & - & If & $"$ & - & Cynwyd, N. Wales. \\
\hline 13 & 2 & " & aratus $(20)$ & ) - & MS. & $"$ & - & $\begin{array}{l}\text { Sholeshook, Pem- } \\
\text { brokeshire. }\end{array}$ \\
\hline 14 & 7 & ", & Brongniärti & ti - & Portl. & , & - & $\begin{array}{l}\text { Desertcreight, Ty- } \\
\text { rone. }\end{array}$ \\
\hline 15 & 2 & " & $"$ & 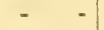 & $"$ & " & - & Below, Llanfyllin. \\
\hline 16 & 1 & " & $”$ & -1 & $"$ & " & - & Chair of Kildare. \\
\hline 17 & 5 & " & " & (20) - & " & $"$ & - & Tyrone. \\
\hline & 3 & ", & conicopthal & almus - & Boeck. & " & & $\begin{array}{l}\text { Llanbedrog, Caer- } \\
\text { narvonshire. }\end{array}$ \\
\hline 19 & 4 & , & " & - & $"$ & $"$ & - & $\begin{array}{l}\text { Cader - dininael, } \\
\text { Cerrig - y-druid- } \\
\text { ion. }\end{array}$ \\
\hline 20 & 2 & " & " & - & " & " & - & $\left\{\begin{array}{l}\text { 1. Gretton. } \\
\text { 2. Acton Scott. }\end{array}\right.$ \\
\hline 21 & 1 & ", & $"$ & - & $"$ & ", & - & Gretton. \\
\hline 22 & 3 & " & $"$ & (20) & " & $"$ & - & Acton Scott. \\
\hline $\begin{array}{l}23 \\
24\end{array}$ & $\begin{array}{l}1 \\
3\end{array}$ & " & Jamesii" & - & Portl. & ”, & - & $\begin{array}{l}\text { Coniston. Water- } \\
\text { Tramore, Word. }\end{array}$ \\
\hline 25 & 3 & " & "... (20 & 0) & & $"$ & 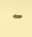 & \\
\hline 26 & 3 & $"$ & Jukesii & - & Salt. & $"$ & - & $\begin{array}{l}\text { W. of Gelli-grin, } \\
\text { Bala. }\end{array}$ \\
\hline 27 & 3 & " & , ? & (20) & & $"$ & - & Tramore. \\
\hline 28 & 1 & $"$ & macroura? & ?- & Sjogren & , & . & $\begin{array}{l}\text { Stockdale Long, } \\
\text { Steddale, Kendal. }\end{array}$ \\
\hline 29 & 1 & $"$ & " & - & ", & ", & - & Acton Scott. \\
\hline 30 & 6 & $"$ & $" \quad($ & (20) -1 & ", & " & - & Coniston. \\
\hline 31 & 1 & $"$ & mimus & ist) $(77)$ & Salt. & $"$ & - & $\begin{array}{l}\text { Peraver, Gorwan } \\
\text { Haven. }\end{array}$ \\
\hline 32 & 4 & , & $\begin{array}{r}\text { mucronatu } \\
\text { connatus, }\end{array}$ & $\begin{array}{l}\text { us (var. } \\
\text {, Salt.) }\end{array}$ & Brong. & " & - & $\begin{array}{l}\text { Pen-y-rhiw, W. of } \\
\text { Bala. }\end{array}$ \\
\hline 33 & 6 & ” & nitidus $(20)$ & & MS. & " & - & $\begin{array}{l}\text { Helm Gill, near } \\
\text { Sedbergh. }\end{array}$ \\
\hline 34 & 1 & ", &,$\quad(20)$ & ) - & & "' & - & $\quad " \quad \quad "$ \\
\hline 35 & 1 & ", & truncato-ca & $\begin{array}{r}\text { audatus } \\
(65)\end{array}$ & Portl. & ", & - & Tyrone. \\
\hline 36 & 1 & ", & " & (20) & , & " & - & Pomeroy, Tyrone. \\
\hline 37 & 2 & " & " & & ", & $"$ & - & $\begin{array}{l}\text { Desertcreight, Ty- } \\
\text { rone. }\end{array}$ \\
\hline 3 & 1 & " & " & - & " & " & - & $"$ \\
\hline $\begin{array}{l}39 \\
40\end{array}$ & $\begin{array}{l}3 \\
2\end{array}$ & $"$ & $"$ & - & $"$ & ”, & - & Lland̈dowror," st. \\
\hline 41 & I & " & $"$ & - & ," & $"$ & - & $\begin{array}{l}\text { Clear's. } \\
\text { resertcreight, Ty- } \\
\text { rone. }\end{array}$ \\
\hline 42 & 1 & & & (20) & & " & & Tyrone. \\
\hline 3 & 1 & $\therefore$ & sp. (20) & - & 10 & ", & - & ", \\
\hline $\begin{array}{l}43 a \\
44\end{array}$ & $\begin{array}{l}1 \\
1\end{array}$ & Amphic & on benevolen & ns & Salt. & $"$ & - & Waterford. \\
\hline 45 & 2 & 5 & pseudo-arti & ticulatus & Portl. & $"$ & - & $\begin{array}{l}\text { Tramore, Water- } \\
\text { ford. }\end{array}$ \\
\hline 46 & I & $"$ & n. sp. (20) & - & - & $"$ & - & $\begin{array}{l}\text { Troutbeck, West- } \\
\text { moreland. }\end{array}$ \\
\hline 47 & 4 & Cheirur & cus bimucron & natus - & Murch. & " & - & $\begin{array}{l}\text { Robeston Wathen, } \\
\text { Haverfordivesh }\end{array}$ \\
\hline
\end{tabular}




\begin{tabular}{|c|c|c|c|c|c|c|}
\hline 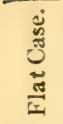 & है & 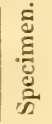 & Name. & $\begin{array}{c}\text { Author } \\
\text { of } \\
\text { Species. }\end{array}$ & Formation. & Locaiity. \\
\hline
\end{tabular}

\begin{tabular}{l|l|l|l|l|l|}
4 & 48 & 2 & Cheirurus bimucronatus - Murch. Caradoc
\end{tabular}

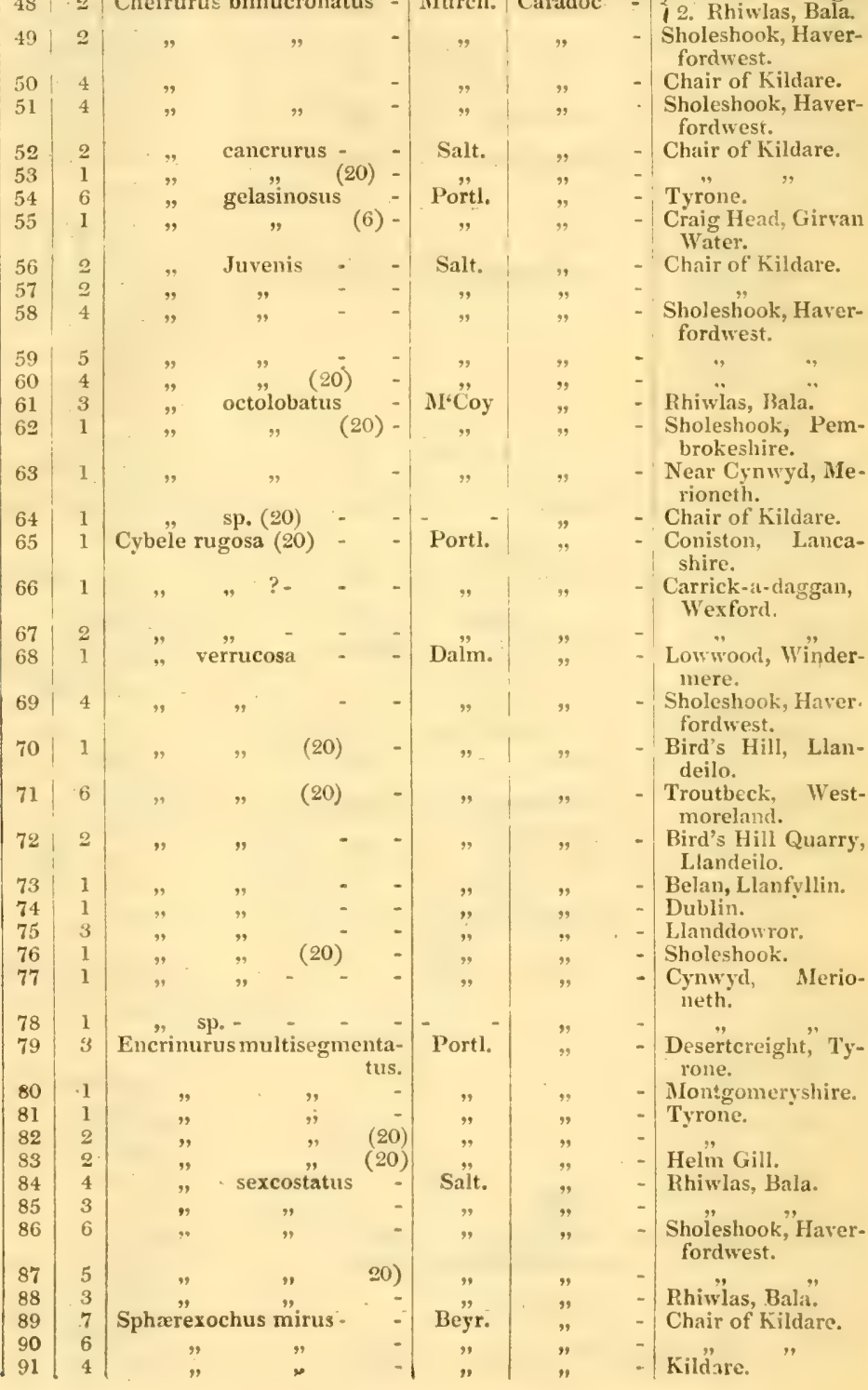




\begin{tabular}{|c|c|c|c|c|c|c|}
\hline 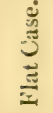 & 苟 & 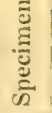 & Name. & $\begin{array}{l}\text { Author } \\
\text { of } \\
\text { species. }\end{array}$ & Formation. & Locality. \\
\hline
\end{tabular}

Crustacea-cont.

\begin{tabular}{l|l|l|l|l|l|l|}
42 & 5 & Sphacexochus mirus (20) - Beyr. & Caradoc - Chair of Kildare.
\end{tabular}

\begin{tabular}{ll|l|l|l}
93 & 1 & " boops - - - Salt. & Caridoc - & - Sholeshook, Haver-
\end{tabular}

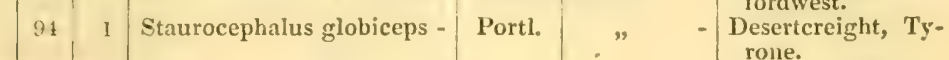

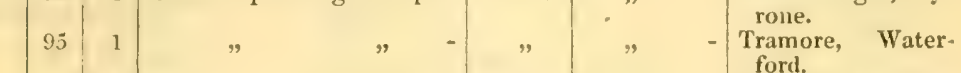

$962 \quad, \quad, \quad(20)$

\begin{tabular}{ccc|cc|c}
97 & 3 & McLareni (65) & Thom. \\
98 & 1 & " & Murchisoni & Barr. \\
99 & 1 & sp. & . &
\end{tabular}

" sp.

Acidaspis bispinosus -

Caractaci -

$\mathrm{MI}^{\circ} \mathrm{Coy}$ Salt.

MS.?

9

Portl. Eileen Vale, near Pomeroy, Tyrone.

Piedmont Glen, Girvan.

- Bryn Bedwog.

- Chair of Kildare.

- Portrane, Dublin.

- Chair of Kildare.

- Gretton, Cardington.

Horderley and Gretton.

- Penwhapple Burn, Girvan.

- Llanddowror.

- Chair of Kildare.

" "

-

- Desertcreight, Tyrone.

- Chair of Kildare.

- Desertcreight, Tyrone.

Portrane, Dublin.

Chair of Kildare.

- Sholeshook, Haverfordwest.

Sholeshook and Acton Scott.

Rhiwlas.

- Acton Scott.

- Cynwyd, Merioneth.

- Tyrone.

- Portrane, Dublln, and Cader Dinmael, N. Wales.

- Sholeshook, Haverforảwest.

Sholeshook and Horderley.

- Desertcreight, Tyrone.

Pont Hafod Gynfon, Llangollen.

- Desertcreight, Tyrone.

- Cynwyd,Merioneth. 


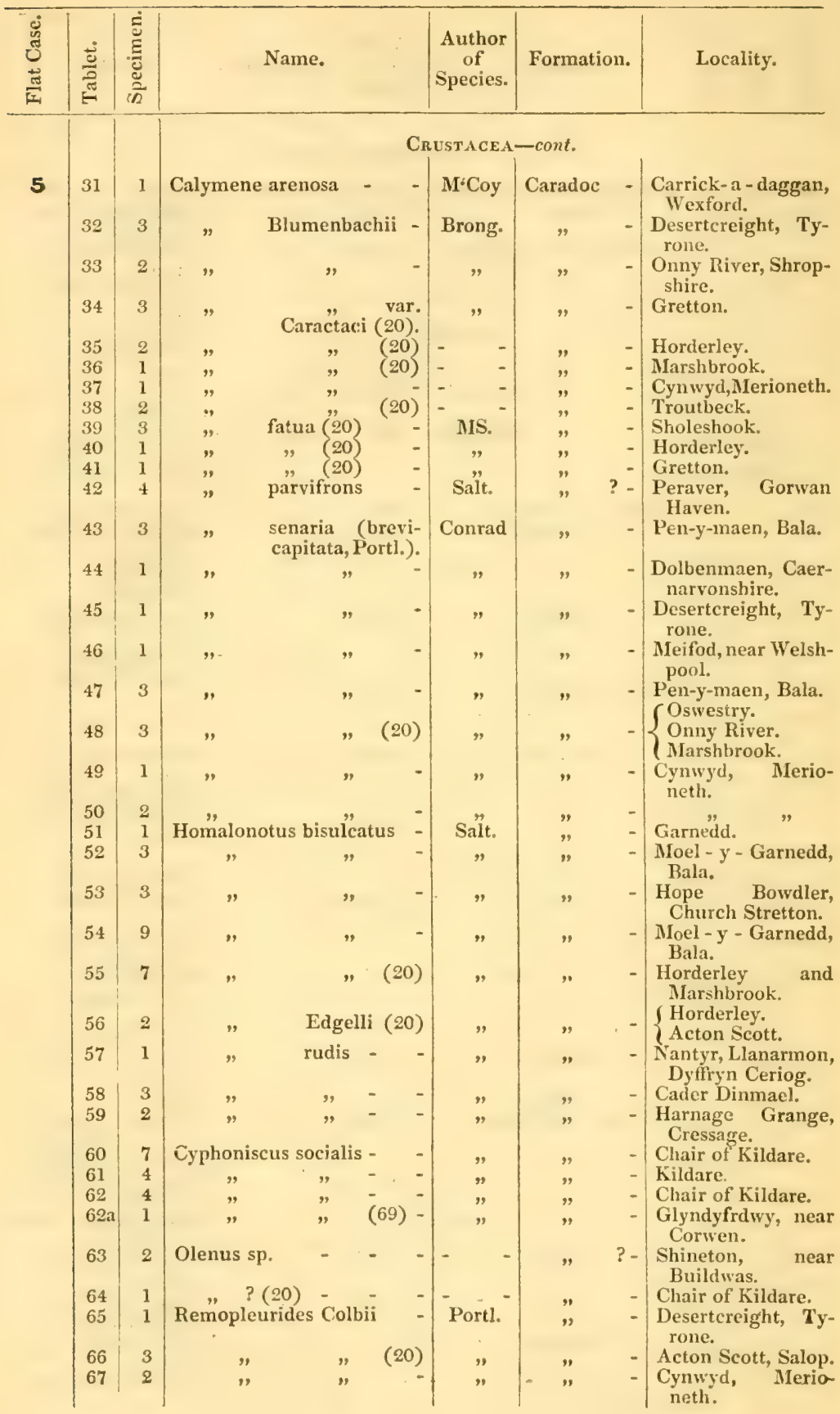




\begin{tabular}{|c|c|c|c|c|c|c|}
\hline 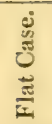 & 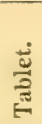 & 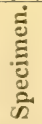 & Name. & $\begin{array}{c}\text { Author } \\
\text { of } \\
\text { Species. }\end{array}$ & Formation. & Locality. \\
\hline
\end{tabular}

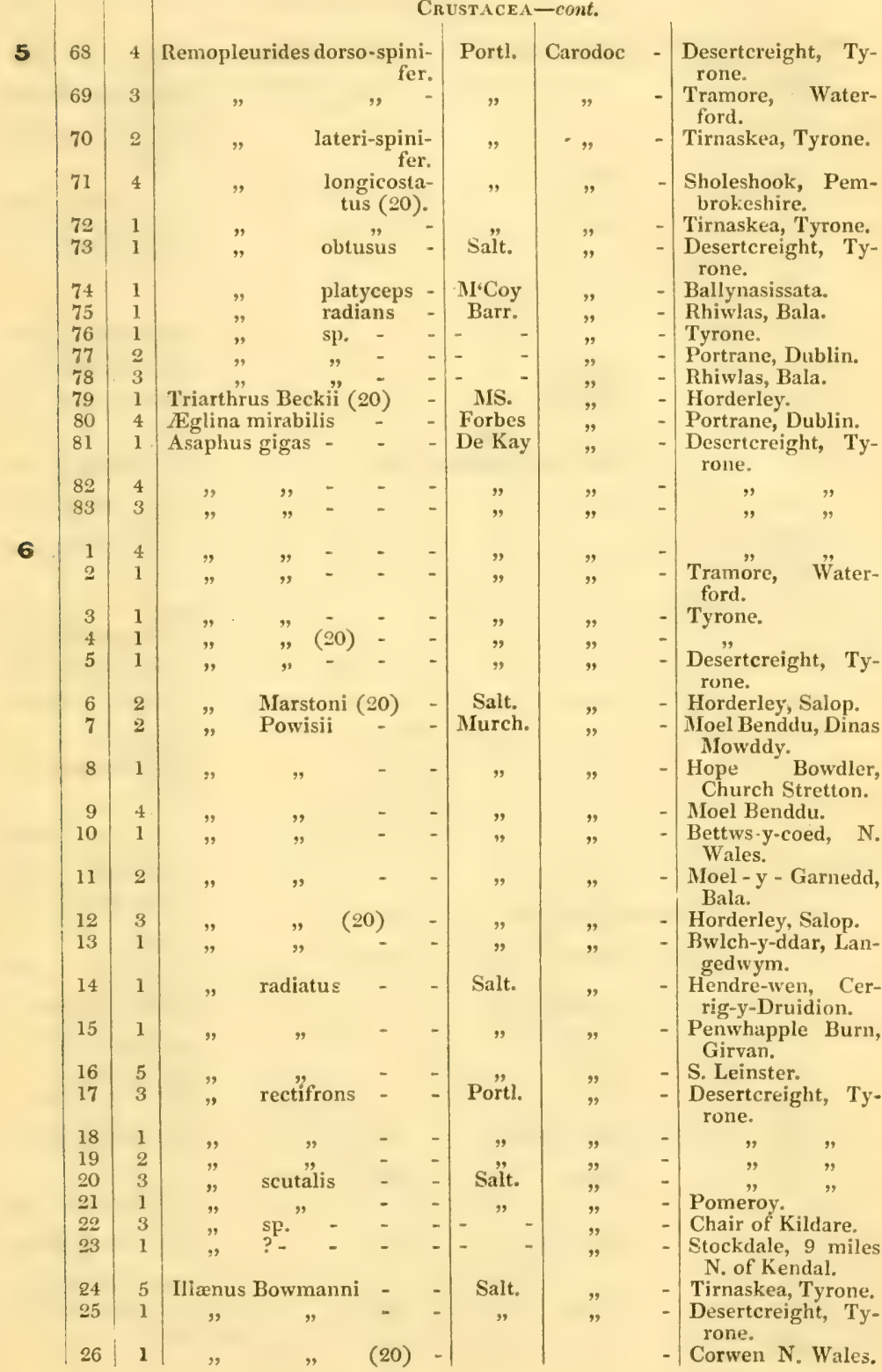




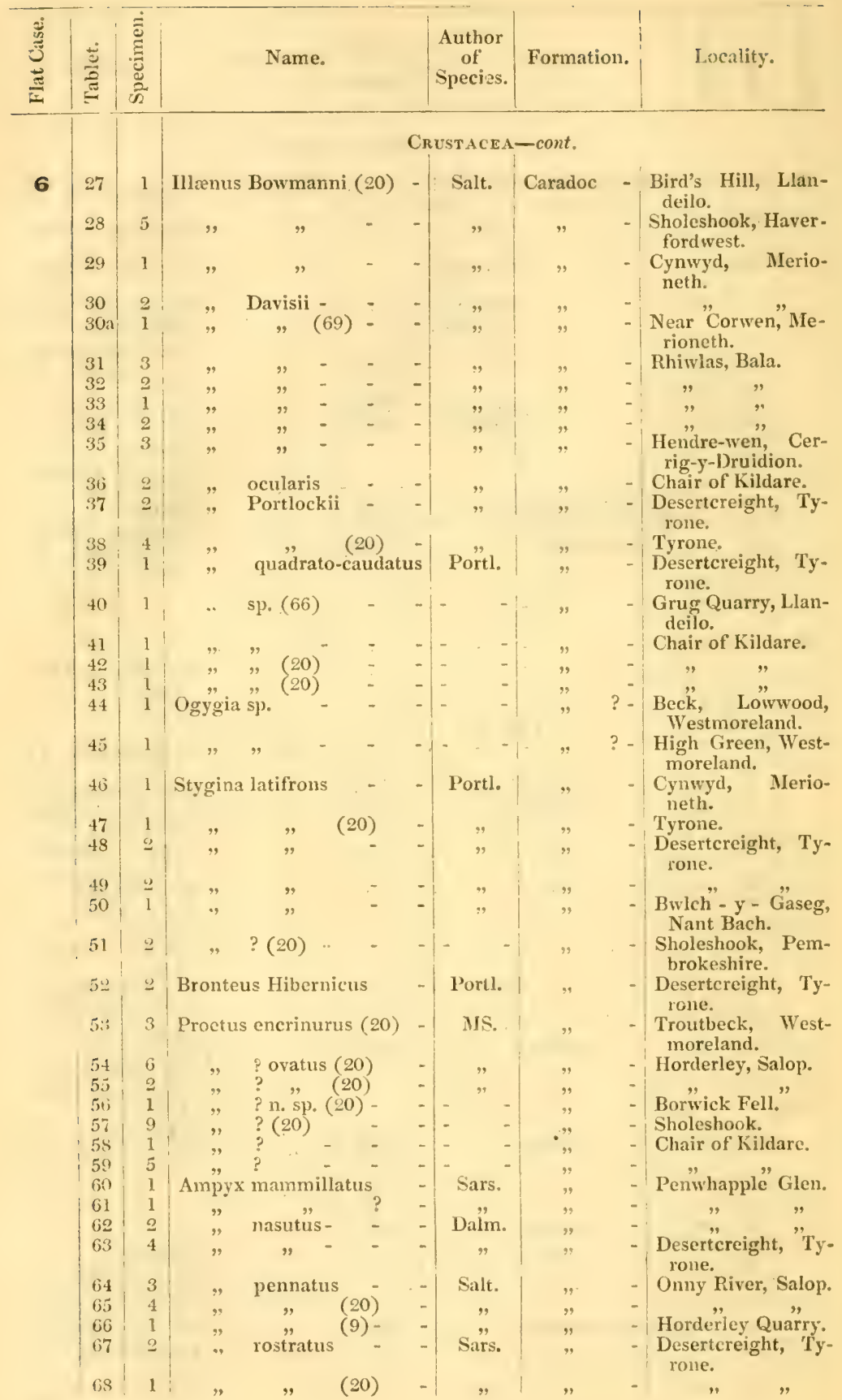




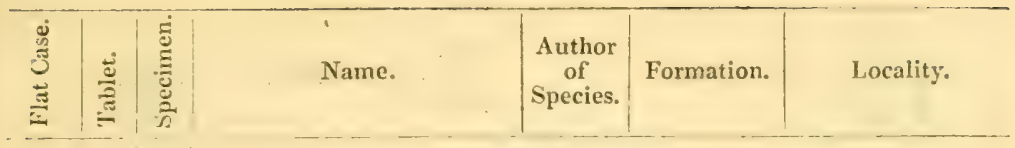

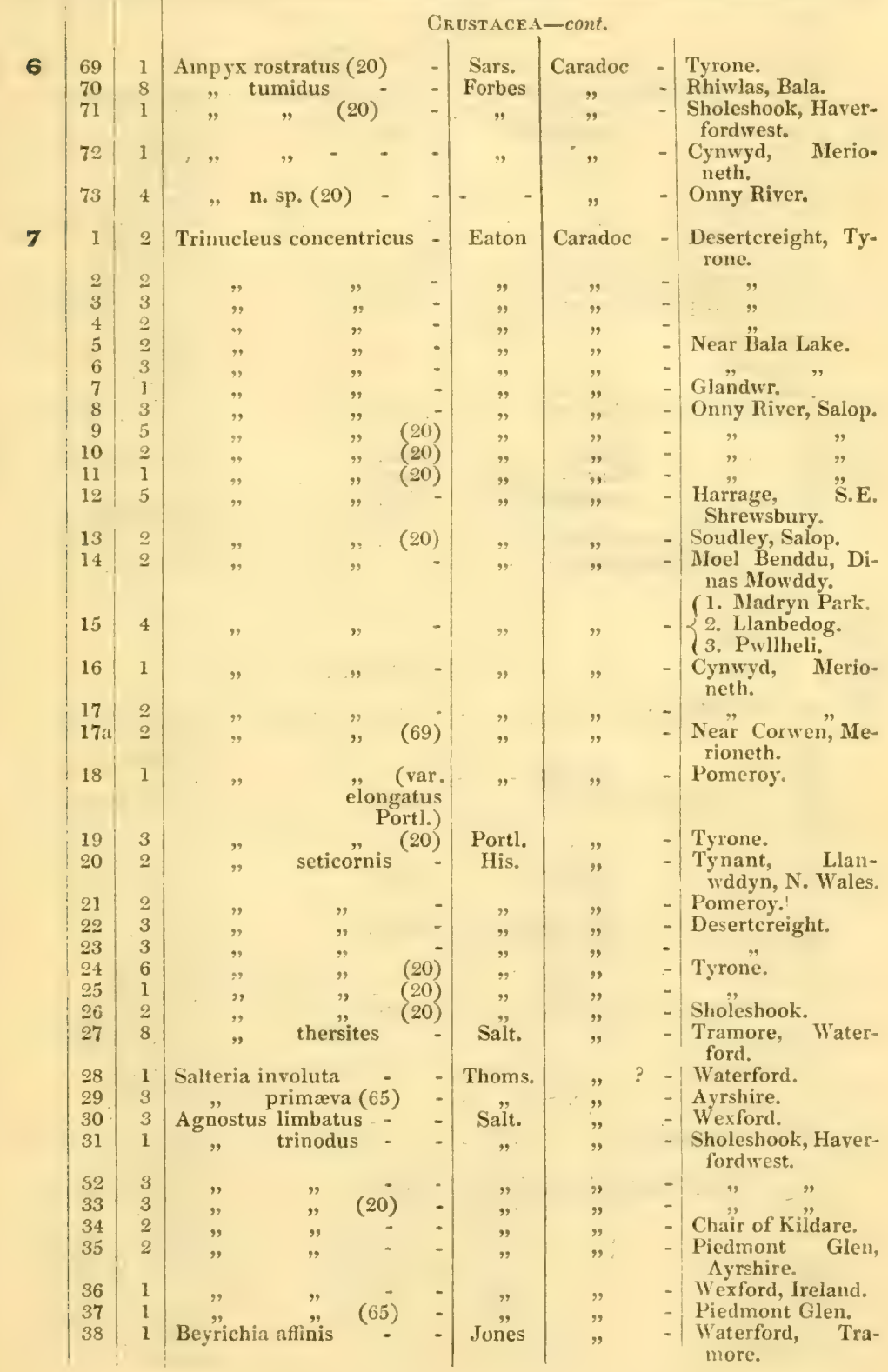




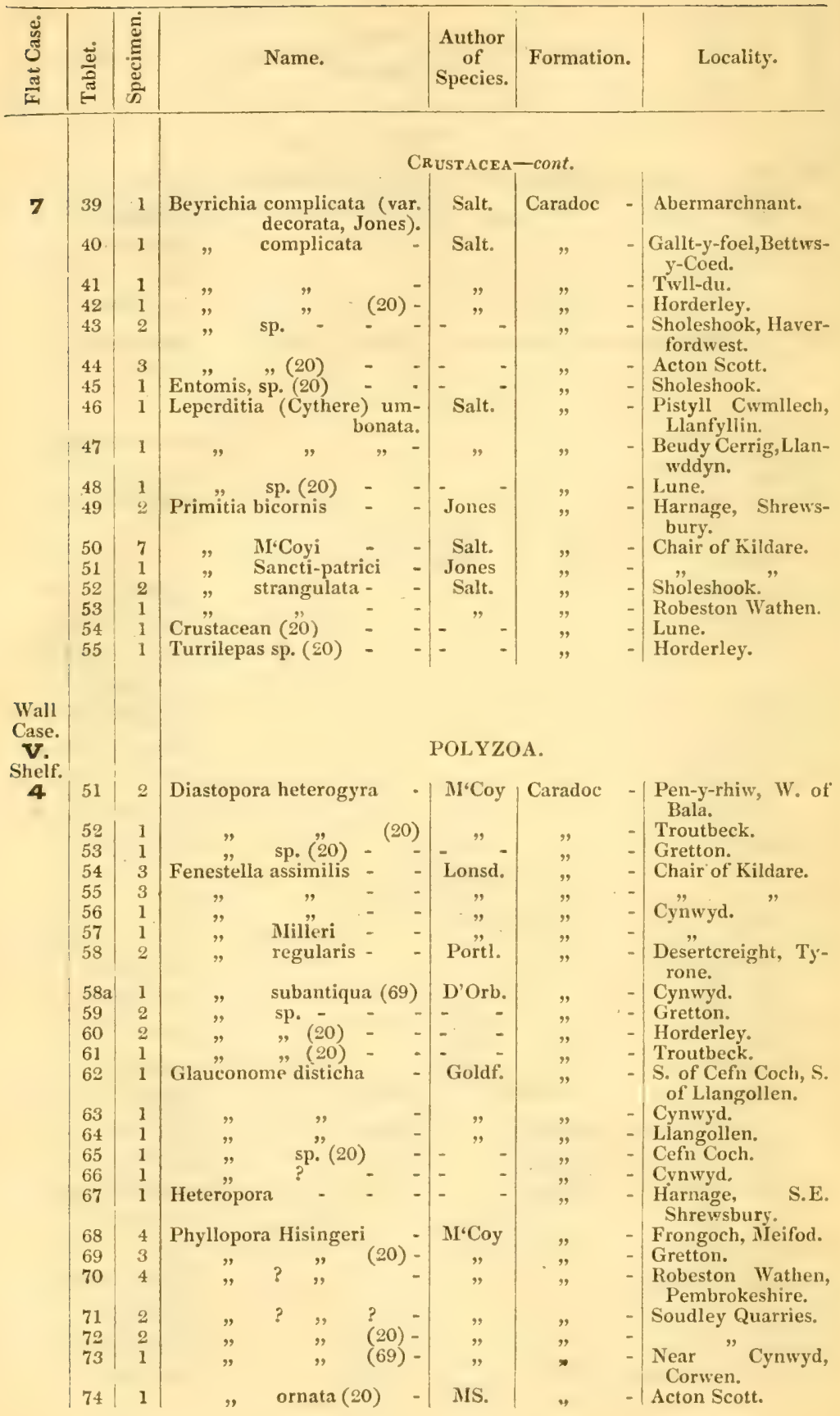




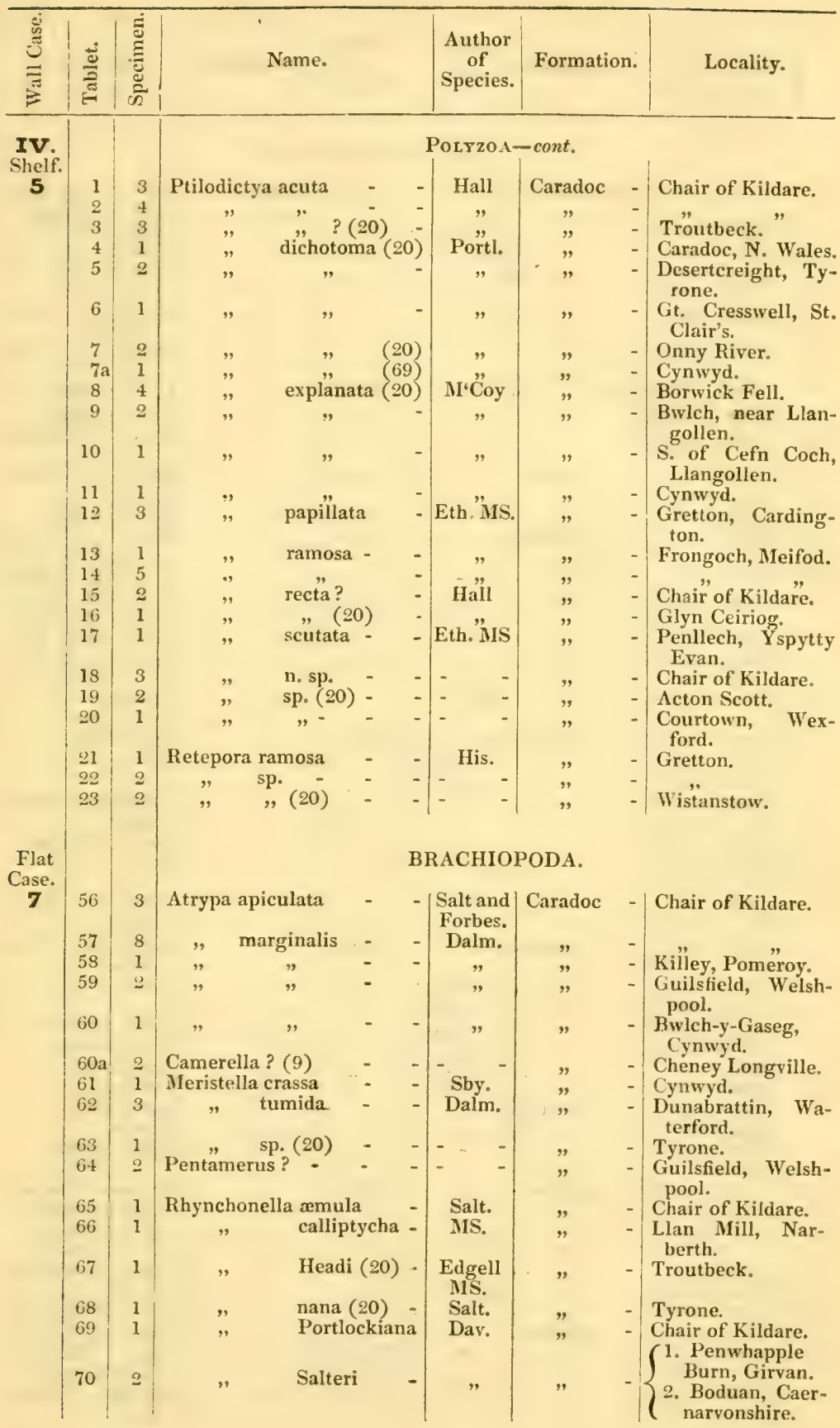




\begin{tabular}{|c|c|c|c|c|c|c|c|c|c|c|c|}
\hline 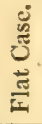 & 苛 & 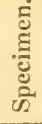 & \multicolumn{5}{|c|}{ Name. } & $\begin{array}{l}\text { Author } \\
\text { of } \\
\text { Species. }\end{array}$ & \multicolumn{2}{|c|}{ Formation. } & Locality. \\
\hline \multirow{36}{*}{7} & & & \multicolumn{9}{|c|}{ BRACHOPODA-cont. } \\
\hline & 71 & 4 & Rhync & honella s] & & - & - & - & Caradoc & & Hendre-wen, Cer- \\
\hline & 72 & 1 & & &,$(68)$ & & - & - & " & - & Craig Head, Girvan. \\
\hline & 73 & 2 & & n & i. sp. & (20) & - & - & $"$ & & Troutbeck. \\
\hline & 74 & 4 & & & p. & - & - & - & $"$ & & $\begin{array}{l}\text { Desertcreight, Ty- } \\
\text { rone. }\end{array}$ \\
\hline & 75 & 1. & - & , & , & - & - & - & " & & $\begin{array}{c}\text { Harnage } \\
\text { Cressage. }\end{array}$ \\
\hline & 76 & 1 & & ? & - & - & - & - & $"$ & & $\begin{array}{l}\text { Dallgwm, near } \\
\text { Bala. }\end{array}$ \\
\hline & 77 & 2 & \multicolumn{4}{|c|}{ Leptæna quinquecostata } & - & $\mathrm{M}^{*} \mathrm{Coy}$ & $"$ & - & Chair of Kildare. \\
\hline & 78 & 4 & , & & & " & - & \multirow{2}{*}{$"$} & $"$ & & Rhiwlas, Bala. \\
\hline & 79 & 1 & $"$ & & & " & - & & " & & $\begin{array}{l}\text { Ty-isaf, Llanged- } \\
\text { wyn. }\end{array}$ \\
\hline & 80 & 2 & $"$ & scissa. & - & - & - & Salt. & " & - & $\begin{array}{l}\text { Sholeshook, Haver- } \\
\text { fordwest. }\end{array}$ \\
\hline & 81 & 3 & " & & - & $\cdots$ & - & \multirow[b]{2}{*}{ Sby. } & $"$ & - & Desertcreight. \\
\hline & 82 & 3 & " & sericea & & - & - & & $"$ & & $\begin{array}{l}\text { Hope Bowdler, } \\
\text { Church Stretton. }\end{array}$ \\
\hline & 83 & 5 & " & " & & - & - & " & , & & Y-Gelli-grin, Bala. \\
\hline & 84 & 1 & $"$ & $"$ & & - & - & " & $"$ & & N.W. of Allt-y- \\
\hline & 85 & 3 & " & ", & - & - & - & " & ", & - & Belau, Llanfyllin. \\
\hline & 86 & 1 & ", & " & & - & - & \multirow{2}{*}{$"$} & , & & Y-Gelli-grin, Bala. \\
\hline & 87. & 2 & ", & " & $(20$ & & - & & 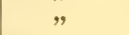 & & Sholeshook. \\
\hline & 88 & i & $"$ & $"$ & $(20)$ & & - & $"$ & $"$ & & Marshbrook. \\
\hline & 89 & 1 & $"$ & $"$ & & - & - & \multirow{2}{*}{$\mathrm{M} \cdot{ }^{\circ} \mathrm{Coy}$} & $"$ & & Cynwyd. \\
\hline & 90 & 4 & $"$ & 'tenuici & incta & & - & & $"$ & & Sholeshook. \\
\hline & 91 & 3 & ", - - & $"$ & & $(20$ & - & 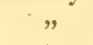 & $"$ & & Tyrone. \\
\hline & 92 & 1 & $"$ & $"$ & & $(20$ & - & \multirow{2}{*}{ " } & " & & Cynwyd, Merio- \\
\hline & 93 & 1 & $"$ & $"$ & & - & - & & ", & & $\begin{array}{l}\text { Cynwyd, Merio- } \\
\text { neth. }\end{array}$ \\
\hline & 94 & 8 & , & $"$ & & - & 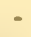 & , & $"$ & - & Chair of Kildare, \\
\hline & 95 & 7 & , & $"$ & & - & - & $"$ & $"$ & - & Bala. \\
\hline & 96 & 1 & ” & $"$ & & - & - & & $"$ & - & Cynwyd, Merio- \\
\hline & 97 & 2 & $"$ & transve & ersali & & $=$ & Dalm. & $"$ & - & $\begin{array}{l}\text { Penwhapple Burn, } \\
\text { Girvan. }\end{array}$ \\
\hline & 98 & 4 & , & , & , & $(20$ & - & , & " & - & Tyrone. \\
\hline & 99 & 1 & $"$ & & & $(20$ & & " & $"$ & & C B Romi \\
\hline & 100 & 3 & , & " & , & $(20$ & - & " & $\eta$ & - & $\left\{\begin{array}{l}\text { 1. Borwick Fen. } \\
\text { 2. Horderley. }\end{array}\right.$ \\
\hline & 101 & 3 & $"$ & , & , & $(20$ & - & " & $"$ & - & Troutbeck. \\
\hline & 102 & 1 & , & & & $(20$ & & $"$ & " & & Tyrone. \\
\hline & 103 & 1 & " & sp. $(16$ & & 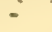 & 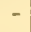 & - & $"$ & - & Llanfyllin. \\
\hline & 104 & 1 & ” & $"(20$ & & 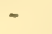 & - & - & " & - & Tyrone. \\
\hline & 105 & 2 & $"$ & $"$ & - & - & - & - & $"$ & - & Rhiwlas, Bala. \\
\hline 8 & 1 & 5 & Orthis & actonia & - & - & - & Sby. & $"$ & - & Robeston Wathen, \\
\hline & 2 & 2 & ", & $"$ & (20) & - & - & " & $"$ & - & $\left\{\begin{array}{l}\text { 1. Troutbeck. } \\
2 \text { Welshpool. }\end{array}\right.$ \\
\hline & 3 & 1 & " & & - & . & - & , & , & - & Moelydd, Oswestry. \\
\hline & 4 & 1 & $"$ & & (20) & - & 1 & $"$ & $"$ & - & Marshbrook. \\
\hline & 5 & 4 & , & & - & - & - & " & $"$ & - & Bryn Bedrog, Bala. \\
\hline & 6 & 2 & 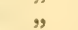 & alternata & & - & - & " & $"$ & 1 & Cheney Longville. \\
\hline & 7 & 4 & $"$ & $"$ & & - & - & $"$ & " & & $\begin{array}{l}\text { Hope Bowdler, } \\
\text { Church Stretton. }\end{array}$ \\
\hline & 8 & 7 & , & $"$ & & - & - & $"$ & $"$ & - & $\begin{array}{l}\text { Harnage, Shrews- } \\
\text { bury. }\end{array}$ \\
\hline
\end{tabular}




\begin{tabular}{|c|c|c|c|c|c|c|}
\hline 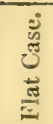 & 总 & 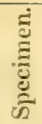 & Name. & $\begin{array}{c}\text { Author } \\
\text { of } \\
\text { Species. }\end{array}$ & Formation. & Locality. \\
\hline
\end{tabular}

8

\begin{tabular}{|c|c|c|c|c|c|c|c|c|c|}
\hline 9 & 1 & Orthi & alternata & - & - & Sby. & Caradoc & - & Cynwyd. \\
\hline 10 & 1 & " & " & - & - & $"$ & ", & - & \\
\hline 1 & 6 & , & " & $\begin{array}{l}\text { Can } \\
\mathrm{II}^{6} \mathrm{Coy}\end{array}$ & & $"$ & $"$ & - & Eglwys Amne, Bala. \\
\hline 12 & 7 & 9 &, & $\eta$ & - & & , & - & Aran Benllyn. \\
\hline & 2 & $"$ & bidens (20) & - & - & $\begin{array}{l}\text { Edgell } \\
\text { MS. }\end{array}$ & " & - & $\begin{array}{l}\text { Helm Gill, near } \\
\text { Sedburgh. }\end{array}$ \\
\hline 4 & 2 & " & biforata - & - & - & Sehloth. & $"$ & - & $\begin{array}{c}\text { Cwm - gwnnen - } \\
\text { uchaf. }\end{array}$ \\
\hline 5 & 1 & " & $(20)$ & - & - & " & و & - & Troutbeck. \\
\hline & 4 & $"$ & " & - & - & , & , & - & Llanfyllin. \\
\hline 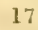 & 7 &, & , & * & - & 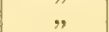 & , & - & Chair of Kildare. \\
\hline & 4 & , & $"$ & - & - & " & , & - & $\begin{array}{l}\text { Bryn } \quad \text { Bedwog, } \\
\text { Bala. }\end{array}$ \\
\hline 19 & 2 & $"$ & (20) & - & - & $"$ & " & - & $\begin{array}{l}\text { Helm Gill. } \\
\left(\begin{array}{c}1 . \text { Robeston Wa- } \\
\text { then. }\end{array}\right.\end{array}$ \\
\hline 20 & 3 & , & biloba & - & - & Linn. & $"$ & - & $\begin{array}{l}\text { 2. Cefn Rhyddan. } \\
\text { 3. Gas Works, } \\
\text { Haverfordwest. } \\
\text { Helm Gill. }\end{array}$ \\
\hline & 4 & $"$ & calligramma & (16) & - & Dalm. & $"$ & - & Moclydd. \\
\hline 2 & 1 &, &, & & - & , & , & - & Bryn Bedwog, Bala. \\
\hline 24 & 2 & " & $"$ & (20) & - & $"$ & " & - & $\begin{array}{l}\text { Grangeegerth, near } \\
\text { Slane. }\end{array}$ \\
\hline 25 & 2 & ", & " & & - & " & $"$ & - & $\begin{array}{l}\text { Llanbabo, Angle- } \\
\text { sea. }\end{array}$ \\
\hline 2 & 1 & " & " $\quad(v$ & $\begin{array}{l}\text { var. pl } \\
\text { a, Sby }\end{array}$ & & " & " & - & Bryn Gwyn, Bala. \\
\hline 27 & 1 & " & $"$ & " & & " & " & - & Bryn Bedwog, Bala. \\
\hline 2 & 1 & $"$ & ") $\quad$ gata & $\begin{array}{l}\text { ar. vi } \\
\text { a, Sby. }\end{array}$ & & , & ", & - & $\begin{array}{l}\text { Cwm - gwnnen - } \\
\text { uchaf. }\end{array}$ \\
\hline 29 & 1 & " & " & , & & $"$ & $"$ & - & $\begin{array}{l}\text { Pwll - y - wrach - } \\
\text { uchaf, Llanwddyn. }\end{array}$ \\
\hline 30 & 1 & " & $n$ & , & & , & " & - & $\begin{array}{l}\text { Desercreight, } \\
\text { rone. }\end{array}$ \\
\hline 31 & 4 & " & crispa - & - & - & $\mathrm{M}^{\circ} \mathrm{Coy}$ & " & - & $\begin{array}{l}\text { Tramore, Water- } \\
\text { ford. }\end{array}$ \\
\hline 2 & 5 & " & $\overline{-}$ & - & - & Dl & " & & Frongoch, Meifod. \\
\hline & 1 & " & elegantula & & - & Dalm. & " & - & Llanfyllin. \\
\hline 34 & 2 & $"$ & $"$ & & - & " & $"$ & - & Moelydd, Oswestry. \\
\hline 3 & 4 & , & " & & - & " & " & - & Horderley. \\
\hline 3 & 5 & $"$ & " & - & - & " & " & -1 & $\begin{array}{l}\text { Madryn Park, Caer- } \\
\text { narvonshire. }\end{array}$ \\
\hline 37 & 2 & و & fallax - & - & - & Salt. & " & - & Desertcreight, $\mathrm{T} y-$ \\
\hline 38 & 2 & " & flabellulum ( & $(20)$ & - & Sby. & $"$ & - & $\left\{\begin{array}{l}\text { 1. Horderley. } \\
\text { 2. Acton Scott. }\end{array}\right.$ \\
\hline 39 & 1 & , & " & & - & $"$ & , & - & $\begin{array}{l}\text { Allt-y-Gader, Llau- } \\
\text { fyllin. }\end{array}$ \\
\hline 40 & 4 & $"$ & $"$ & & .* & ", & $"$ & - & $\begin{array}{l}\text { Boduan, Caernar- } \\
\text { vonshire. }\end{array}$ \\
\hline 4] & 2 & " & $"$ & & - & $"$ & " & - & $"$ \\
\hline & $\begin{array}{l}6 \\
2\end{array}$ & $"$ & $"$ & & - & $"$ & $"$ & $=$ & Meifod, Welsh"pool. \\
\hline & 1 & " & 9 & & - & , & " & - & $\begin{array}{l}\text { Cynwyd, Merio- } \\
\text { neth. }\end{array}$ \\
\hline 45 & 4 & " & " tifida & $\begin{array}{l}\text { ar.mu } \\
\text { a, Salt }\end{array}$ & & " & " & - & $\begin{array}{l}\text { Cefn Llyfnog, Llan- } \\
\text { fyllin. }\end{array}$ \\
\hline & 2 & " & Hirnantensis & - & - & $\mathrm{I}^{6} \mathrm{Coy}$ & $"$ & - & Aber Hirnant. \\
\hline & 2 & " & " & - & $\cdot$ & , & $"$ & - & \\
\hline
\end{tabular}




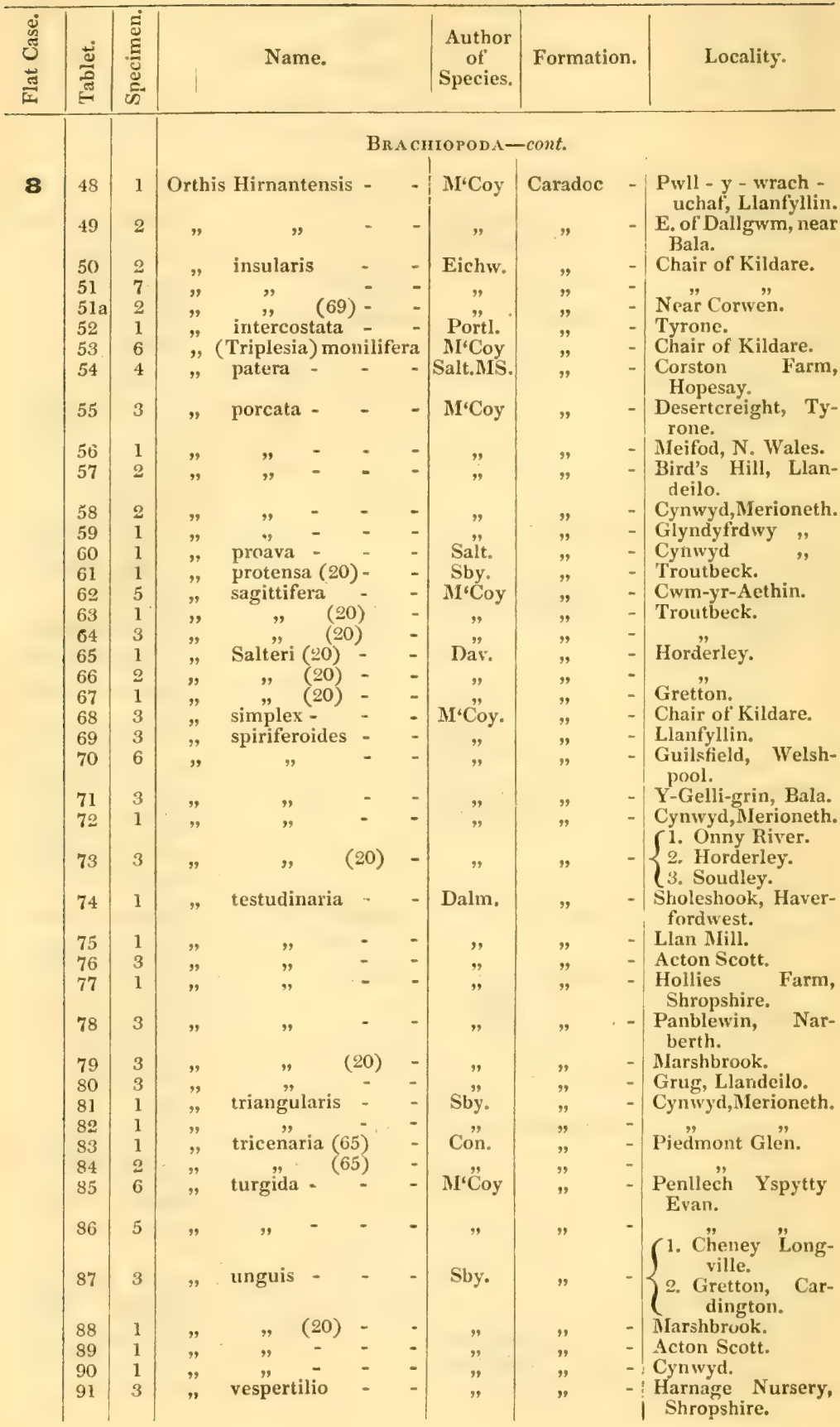




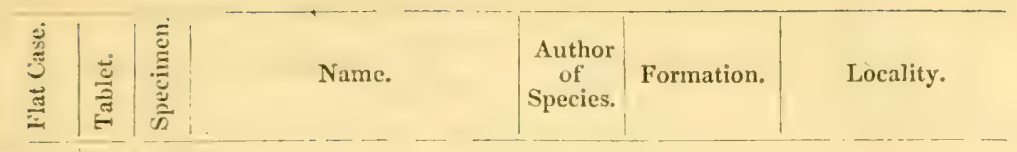

8

3

BRACHIOHODA-cont.

\begin{tabular}{l|l|l|l} 
Orthis vespertilio - - Sby. & Caradoc - E. of Bala Lake.
\end{tabular}

sp. (16)

$$
\text { , sp. }
$$

Orthisina adscendens " " Strophomena antiquat arenacea euglypha expansa

$$
",
$$
Acton Burnell.

- Gretton.Cardington.

- Bala.

Acton Scott.

Cynwyd,Merioneth. -

Moelydd, Oswestry.

Cilgwyn, Llandovery.

- Troutbeck.

- Desertcreight, Tyrone.

- Moelydd, Oswestry. Pen-yr-rhiw, Bala. Cynwyd.

- "

Chaï of Kildare. Huntley Hill.

- Gunwick Mill, Alfrick.

Desertcreight, Tyrone.

Chair of Kildare.

Newtown, Water ford.

- Tyrone.

Acton Scott.

- Soudley, Church Stretton.

- Harnage, Shrewsbury.

- Bardahessiagh, Tyrone.

- Horderley.

- Meifod, Welshpool.

- Pentref, Abermarchnant.

- Cheney Longville.

- Tirnaskea, Tyrone.

Acton Scott.

Onny Itiver.

Troutbeck.

Tyrone.

- Chair of Kildare.

- E. of Bala Lake.

- Celyn Meifod, Welsl pool.

- N.W. of I'wll ywrach-isaf, Llanwddyn.

- Pont Hafod Gynfor, Llangollen.

- Bird's Hill.

- Cynwyd,Merioneth. 


\begin{tabular}{|c|c|c|c|c|c|c|c|c|c|c|c|}
\hline 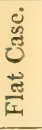 & $\underset{\frac{0}{0}}{\frac{0}{\pi}}$ & 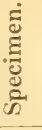 & \multicolumn{5}{|c|}{ Name. } & $\begin{array}{c}\text { Author } \\
\text { of } \\
\text { Species. }\end{array} \mid$ & \multicolumn{2}{|c|}{ Formation. } & Locality. \\
\hline \multirow{44}{*}{9} & \multirow[b]{2}{*}{43} & \multirow[b]{2}{*}{1.} & \multicolumn{9}{|c|}{ BrACHIOPONA-cont. } \\
\hline & & & \multicolumn{5}{|c|}{ Strophomena rhomboidalis } & Wilck. & Caradoc & - & Desertereight, $\mathbf{T y}$ - \\
\hline & 44 & 2 & " & & & $"$ & & $"$ & , & - & Chair of Kildare. \\
\hline & $\begin{array}{l}45 \\
46\end{array}$ & $\begin{array}{l}1 \\
1\end{array}$ & , & & & $"(20$ & & $"$ & $"$ & - & Troutbeck," \\
\hline & 47 & 1 & " & & & $" 2(20$ & & $"$ & $"$ & - & \\
\hline & 48 & 1 & " & & &,$(20$ & & $"$ & $"$ & - & Coniston. \\
\hline & 49 & 1 & " & & & & & $"$ & ", & - & Cheney Longville. \\
\hline & 50 & 1 &, & & & " & & $"$ & $"$ & - & Marshbrook. \\
\hline & 51 & $\begin{array}{l}1 \\
2\end{array}$ & , & & & $"(20$ & & $"$ & $"$ & - & Acton Scott. \\
\hline & 52 & 2 & & & & $"$ & & $"$ & $"$ & - & $\begin{array}{l}\text { Gretton, Carding- } \\
\text { ton. }\end{array}$ \\
\hline & $\begin{array}{l}53 \\
54\end{array}$ & $\begin{array}{l}1 \\
1\end{array}$ & Crania & divaric & ata & $"(20$ & & $M^{6} \mathrm{Coy}$ & $"$ & - & Chair of Kildare. \\
\hline & 55 & 7 & $"$ & $"$ & & - & & $"$ & $"$ & - & , \\
\hline & 56 & 1 & $"$ & sp. "2 & & - & & $"$ & $"$ & - & Y-Gelli-grin. \\
\hline & 57 & 1 & $"$ & $\begin{array}{l}\text { sp. }(2 \\
.(2 r\end{array}$ & & - & & - & $"$ & - & Gretton. \\
\hline & 58 & $\begin{array}{l}1 \\
2\end{array}$ & $"$ & $"(2$ & & $\overline{-}^{-}$ & - & & ", & - & Horderley. \\
\hline & $\begin{array}{l}59 \\
60\end{array}$ & $\begin{array}{l}2 \\
1\end{array}$ & Discina & cance & llata & (20) & - & Salt. MS. & ”, & - & $\begin{array}{l}\text { Onny River. } \\
\text { Horderley. }\end{array}$ \\
\hline & 61 & 1 & ", & " & & (20) & - & 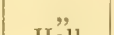 & $"$ & - & Marshbrook. \\
\hline & 62 & 3 & $"$ & crassa & - & - & - & Hall & $"$ & - & Penwhapple Glen. \\
\hline & 63 & 2 & $"$ & lingul & form & & - & $M "$ & $"$ & - & Gretton. \\
\hline & $\begin{array}{l}64 \\
65\end{array}$ & $\begin{array}{l}2 \\
1\end{array}$ & $"$ & oblon & gator & mis & - & Mall & $"$ & - & $\begin{array}{l}\text { Chair of Kildare. } \\
\text { Tyrone. }\end{array}$ \\
\hline & 66 & 2 & " & $"$ & & - & - & Portl. & $"$ & - & $\begin{array}{l}\text { Desertcreight, } \mathrm{Ty}- \\
\text { rone. }\end{array}$ \\
\hline & 67 & 3 & $"$ & " & & - & - & " & $"$ & - & $", \quad$, \\
\hline & 68 & 3 & $"$ & $"$ & & - & - & $"$ & $"$ & - & (1. Acton "Scott, \\
\hline & 69 & 2 & $"$ & " & & (20) & - & " & $"$ & - & $\left\{\begin{array}{l}\text { Salop. } \\
\text { 2, Horderley, Sa- } \\
\text { lop. }\end{array}\right.$ \\
\hline & 70 & 1 & $"$ & perrus & gata & - & - & $\mathrm{M} \cdot \mathrm{Coy}$ & $"$ & - & Chair of Kildare. \\
\hline & 71 & I & $"$ & $"$ & & - & - & $"$ & $"$ & - & $\begin{array}{l}\text { Desertcreight, Ty- } \\
\text { rone. }\end{array}$ \\
\hline & 72 & 1 & $"$ & $"$ & & - & - & $"$ & , & - & $\begin{array}{l}\text { Hope Bowdler, near } \\
\text { Church Stretton. }\end{array}$ \\
\hline & 73 & 4 & $"$ & punct: & & - & - & Sby. & " & - & $\begin{array}{l}\text { Cheney Longville } \\
\text { Lane. }\end{array}$ \\
\hline & 74 & 3 & $"$ & $"$ & & $\cdot$ & - & $"$ & " & - & Hope Bowdler, near \\
\hline & 75 & 1 & $"$ & Siluri & ana $(6$ & 67) & - & Dav. & $"$ & - & $\begin{array}{l}\text { Church Str } \\
\text { Horderley. }\end{array}$ \\
\hline & 76 & 2 & $"$ & sp. $(20$ & & - & . & ” & $"$ & - & Tyrone. \\
\hline & 77 & 2 & ci." & $\ddot{M}(20$ & & - & - & & $"$ & - & Borwick Fell. \\
\hline & 78 & 3 & $\begin{array}{l}\text { Siphon } \\
\text { Lingul: }\end{array}$ & treta $n$ & nicula & a (13) & - & $\begin{array}{l}\text { M'Coy } \\
\text { Porti. }\end{array}$ & $"$ & - & $\begin{array}{l}\text { Conway Castie. } \\
\text { Desertcreight. }\end{array}$ \\
\hline & 79 & 1 & & a brevis & - & - & - & Portl. & $"$ & -1 & $\begin{array}{l}\text { Desertcreight, } \mathrm{Ty} \text { - } \\
\text { rone. }\end{array}$ \\
\hline & 80 & 5 & " & lata? & & - & - & Sby. & " & - & Plas-hen, Pwllheli. \\
\hline & 81 & 2 & " & ovata & & - & - & M.Coy & $"$ & - & Desertcreight. \\
\hline & 82 & 1 & $"$ & $"$ & - & - & - & $"$ & $"$ & - & $\begin{array}{l}\text { Duncannon, Wex- } \\
\text { ford. }\end{array}$ \\
\hline & 83 & 1 & $"$ & $"$ & - & 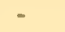 & - & , & " & - & Bryn Bedwog, Bala. \\
\hline & 84 & 2 & $"$ & " & - & - & - & $"$ & $"$ & - & N. of Pentre-llymru. \\
\hline & 85 & 2 & " & $"$ & & & - & $"$ & $"$ & - & $\begin{array}{l}\text { BwIch-y-Gaseg, nr. } \\
\text { Corwen. }\end{array}$ \\
\hline & 86 & 1 & $"$ & $"$ & (20) & - & - & $"$ & " & -1 & Marshbrook, Salop. \\
\hline & 87 & 1 & $"$ & $"$ & $(20)$ & 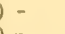 & - & " & $"$ & - & Horderley, Salop. \\
\hline & $\begin{array}{l}88 \\
89\end{array}$ & $\begin{array}{l}1 \\
1\end{array}$ & $"$ & tenuis & $\begin{array}{l}(20) \\
\text { granu }\end{array}$ & & - & $"$ & $"$ & - & Pwll-y \\
\hline & & & & & & & & & & & Llanwddyn. \\
\hline
\end{tabular}




\begin{tabular}{|c|c|c|c|c|c|c|}
\hline 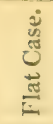 & 离 & 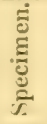 & Name. & $\begin{array}{l}\text { Author } \\
\text { of } \\
\text { Species. }\end{array}$ & Formation. & Locality. \\
\hline
\end{tabular}

IBRACHOPODA-cont.

$9|90|$ I $\quad$ Lingula tenuigranulata $-\mid \mathrm{I}^{\circ} \mathrm{Coy} /$ Caradoc $-\mid \begin{gathered}\text { Pistyll Cwmllech, } \\ \text { Llanfyllin. }\end{gathered}$ 91 | 1 - _ - Goetre

Welshpool.

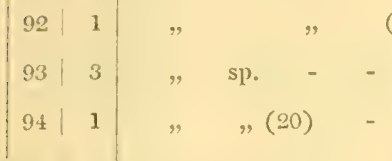

Drawer.

\begin{tabular}{|c|c|c|c|c|c|c|}
\hline 1 & 1 & Leptrer & a sericea & .. & - & Sby. \\
\hline 2 & 1 & $"$ & " & - & - & $"$ \\
\hline 3 & I & " & " & - & - & " \\
\hline 4 & 3 & $"$ & " $(20)$ & ) & $\dot{-}$ & M'C̈) \\
\hline 5 & 5 & " & tenuicincta & $2(20)$ & - & M.coy \\
\hline$\frac{0}{7}$ & 2 & " & transversali & is $(16)$ & i) & Dal̆m. \\
\hline $7 a$ & 3 & Orthis & Actonix & $\%$ & - & $\begin{array}{l}\text { Daim. } \\
\text { Sby. }\end{array}$ \\
\hline S & 1 & $"$ & , (20) & - & - & $"$ \\
\hline 9 & 4 & $"$ & alternata & - & $*$ & $"$ \\
\hline 10 & 3 & " & , $\quad(20)$ & - & - & " \\
\hline 11 & 4 & $"$. & $"$ & - & - & $"$ \\
\hline 12 & 1 & " & - & - & - & "' \\
\hline 15 & 2 & $"$ & $"$ & - & - & $"$ \\
\hline 14 & 1 & $"$ & biforata (20) & - & - & Schloth. \\
\hline 15 & 1 & $"$ & $(20)$ & - & - & 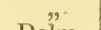 \\
\hline 16 & 1 & $"$ & calligranma & $a(70)$ & - & Dalm. \\
\hline 17 & 2 & " & $"$ & $(20)$ & - & " \\
\hline 18 & 1 & " & " & - & - & " \\
\hline 19 & 2 & ", & " & (20) & - & $"$ \\
\hline 20 & 6 & ", & " & $\overline{-}$ & - & $"$ \\
\hline 2 & 1 & " & " & (20) & - & " \\
\hline 22 & 1 & " & $"$ & (20) & - & $"$ \\
\hline 23 & 1 & " & elegantula & - & - & " \\
\hline 2 & 3 & " & " ( & 9) & - & Sh= \\
\hline 25 & 3 & " & flabellulum & - & - & Sby. \\
\hline 26 & 1 & " & & - & - & \\
\hline 27 & 1 & " & porcata - & - & - & $\mathrm{M}^{\circ} \mathrm{Coy}$ \\
\hline 28 & 1 & $"$ & $(20)$ & - & - & " \\
\hline 25 & 2 & , & $(20)$ & - & - & " \\
\hline 30 & 5 & " & " (20) & - & - & " \\
\hline 31 & 5 & " & spiriferoides & s - & - & " \\
\hline 32 & 1 & " & "' & - & - & " \\
\hline 33 & 2 & " & testudinaria & - & - & Dalm. \\
\hline 34 & 1 & " & " & (20) & - & " \\
\hline 35 & 4 & , & $"$ & - & - & " \\
\hline 36 & 1 & , & " & $(20)$ & - & $"$ \\
\hline 37 & 3 & " & vespertilio & - & - & sliy. \\
\hline 38 & 3 & "थ & و" & - & - & " \\
\hline 39 & 1 & " & " & - & - & $"$ \\
\hline 40 & 3 & " & & (20) & - & " \\
\hline 41 & 2 & ', & sp. $(20)$ & - & - & -1 \\
\hline
\end{tabular}

- Cheney Longville, Salop.

- Desertereight, Tyrone.

Horderley.

Pwll-y-wrach-isaf.

Rhydwen, Bala.

- Llan y cil, Bala.

- Troutbeck.

- Sholeshook.

- Tyrone.

- Moelydd, Oswestry.

- Bala.

- Troutbeck.

- Madryn Park.

- Horderley.

- Pentre-llymru, Llanfyllin.

- Cynwyd, Merioneth.

- Portrane and Lambay, Dublin.

- Gretton.

- Tyrone.

- Knockmahon, Waterford.

- Coniston, cashire.

Y-Gelli-grin, Bala.

Sholeshook.

- Bryn Bedwog,Bala.

- Onny River.

- Coniston, cashire.

Gretton.

Cheney Longville.

- Boduan, Caernarvonshire.

- Meifod.

- Bwlch-y-Ciban.

Troutbeck.

Tyrone.

Pont Rhiwedog.

Bala.

Cheney Longville.

Marshbrook.

- Grug, Llandeilo.

- Horderley, Salop.

- Harnage, S.E. of Shrewsbury.

- Corston Farm, Hopesay.

- Onnj River.

- Tyrone. 


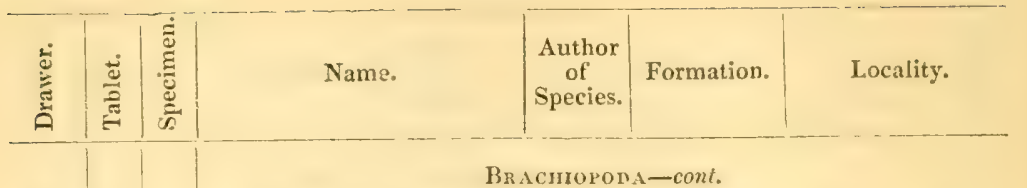

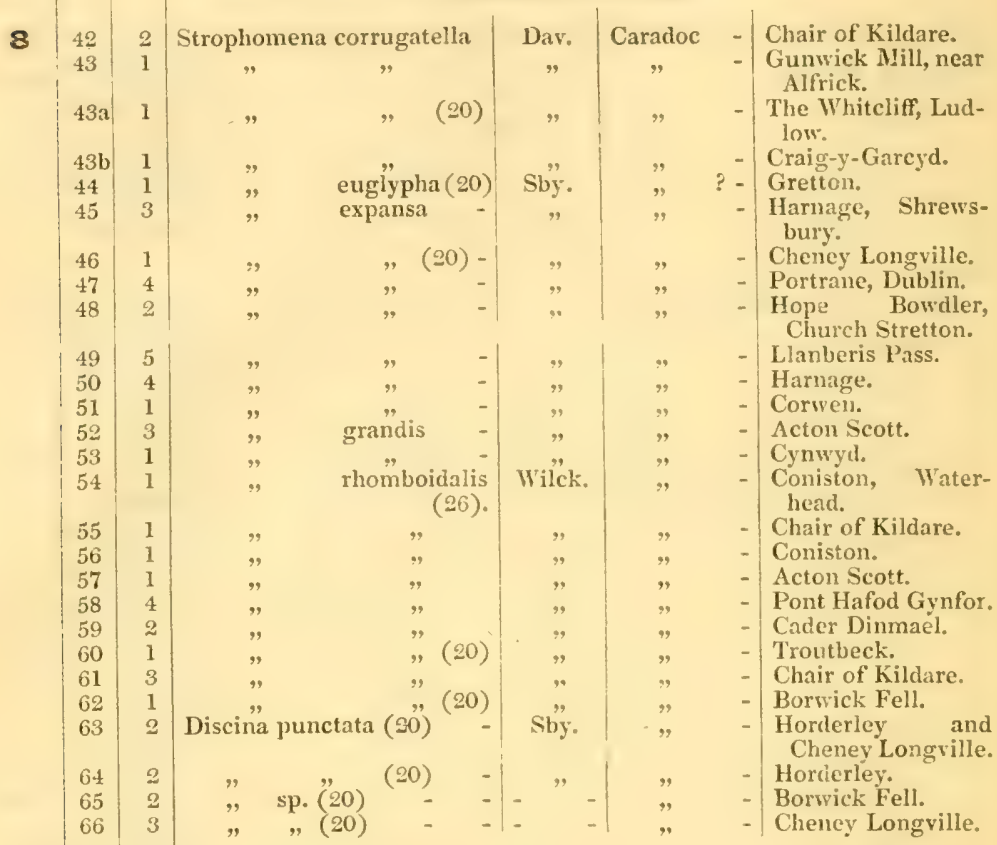

\section{LAMELLIBRANCHIATA.}

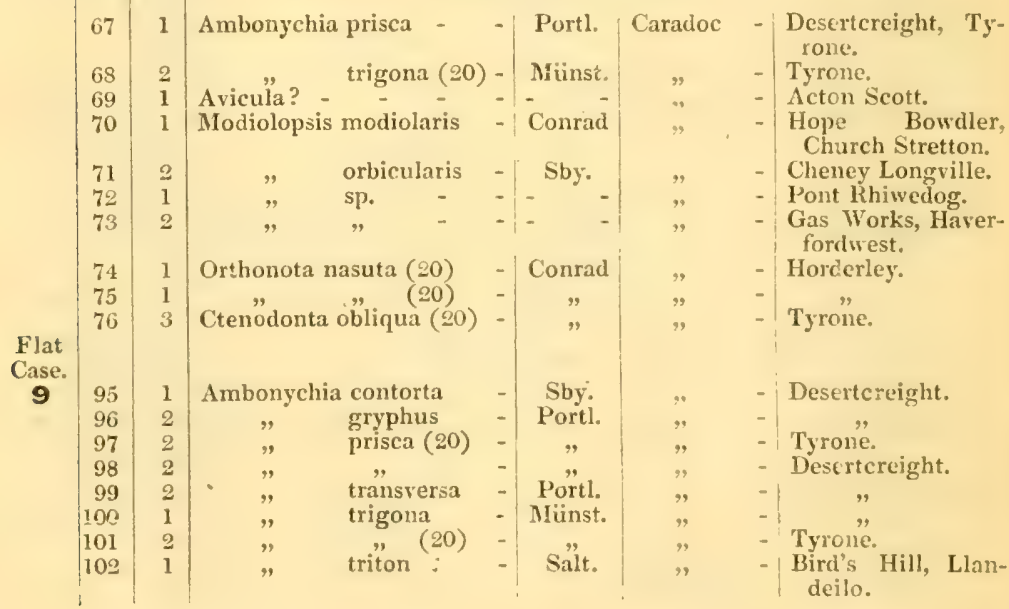




\begin{tabular}{|c|c|c|c|c|c|c|}
\hline 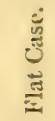 & हैं & 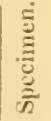 & Name. & $\begin{array}{c}\text { Author } \\
\text { of } \\
\text { Species. }\end{array}$ & Formation. & l.ocality. \\
\hline
\end{tabular}

\section{LAMEILIBRANCHIATA-cont.}

9

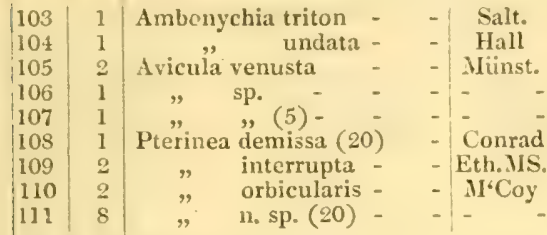

\begin{tabular}{|c|c|c|c|c|}
\hline 1 & 2 & Modiolopsis & Brycei - & Portl. \\
\hline 2 & 1 & , & $"$ & \\
\hline 3 & 1 & ," & curta $(20)$ & $\begin{array}{l}\text { Edgell } \\
\text { MS. }\end{array}$ \\
\hline 1 & 2 & " & elliptica? & Hall \\
\hline 5 & 3 & " & expansa - & Portl. \\
\hline 6 & 1 & " & , (20) - & \\
\hline 7 & 2 & $"$ & modiolaris - & Conrad \\
\hline 8 & 1 & ", & $(20)$ & " \\
\hline 9 & 1 & $"$ & $(20)$ & \\
\hline 10 & 2 & , & orbicularis - & Sby. \\
\hline & 1 & , & $\quad, \quad-$ & \\
\hline & 2 & , & $\begin{array}{l}\text { (Anodontopsis) } \\
\text { securiformis. }\end{array}$ & M'Coy \\
\hline
\end{tabular}

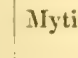




\begin{tabular}{|c|c|c|c|c|c|c|}
\hline 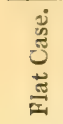 & है & 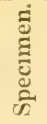 & Name. & $\begin{array}{c}\text { Author } \\
\text { of } \\
\text { Species. }\end{array}$ & Formation. & Locality. \\
\hline
\end{tabular}

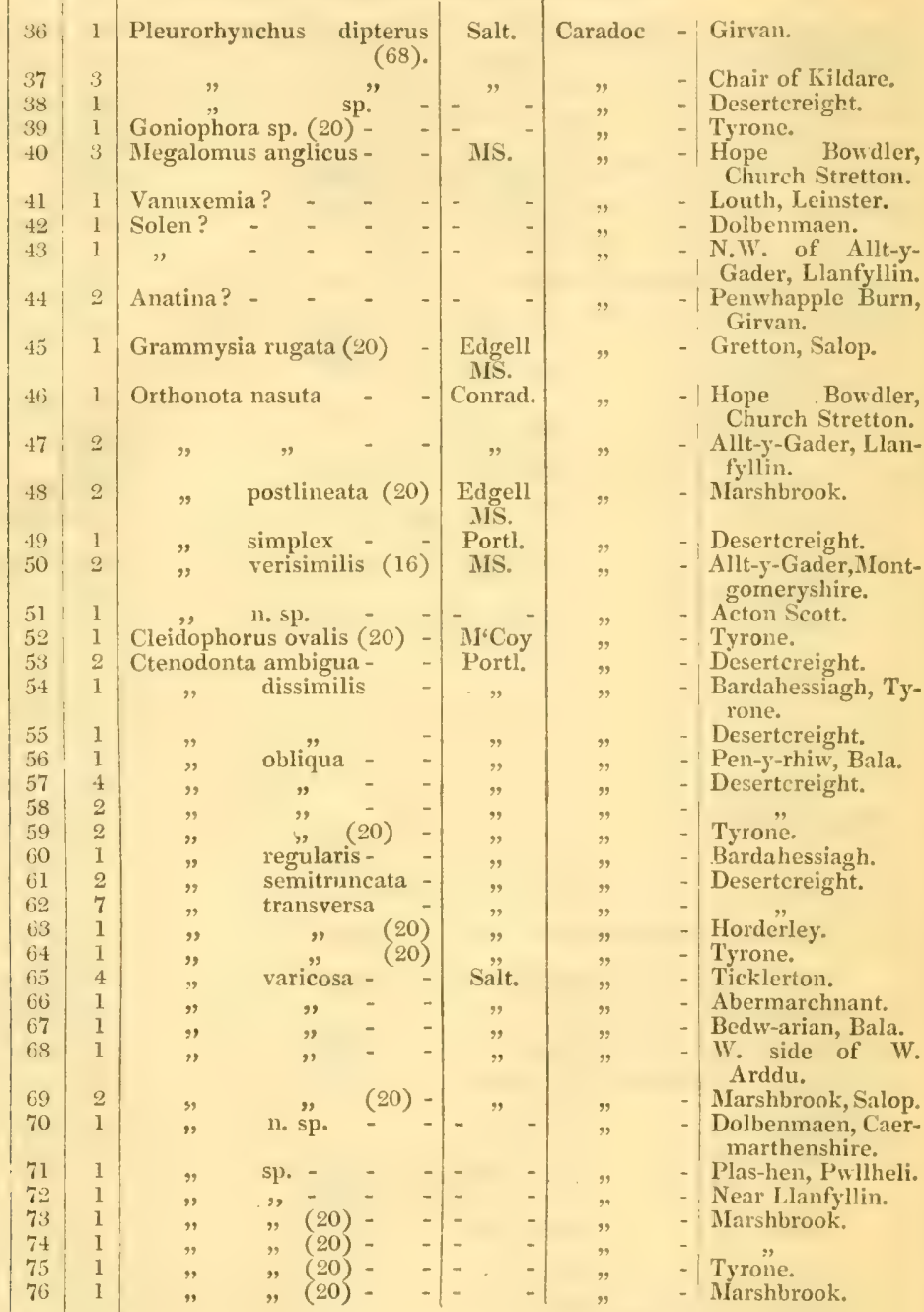

GASTEROPODA.

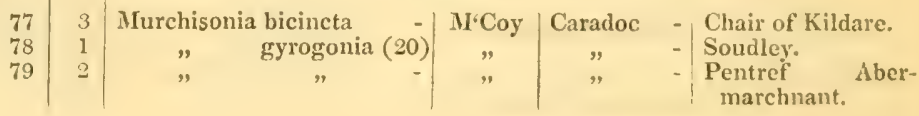




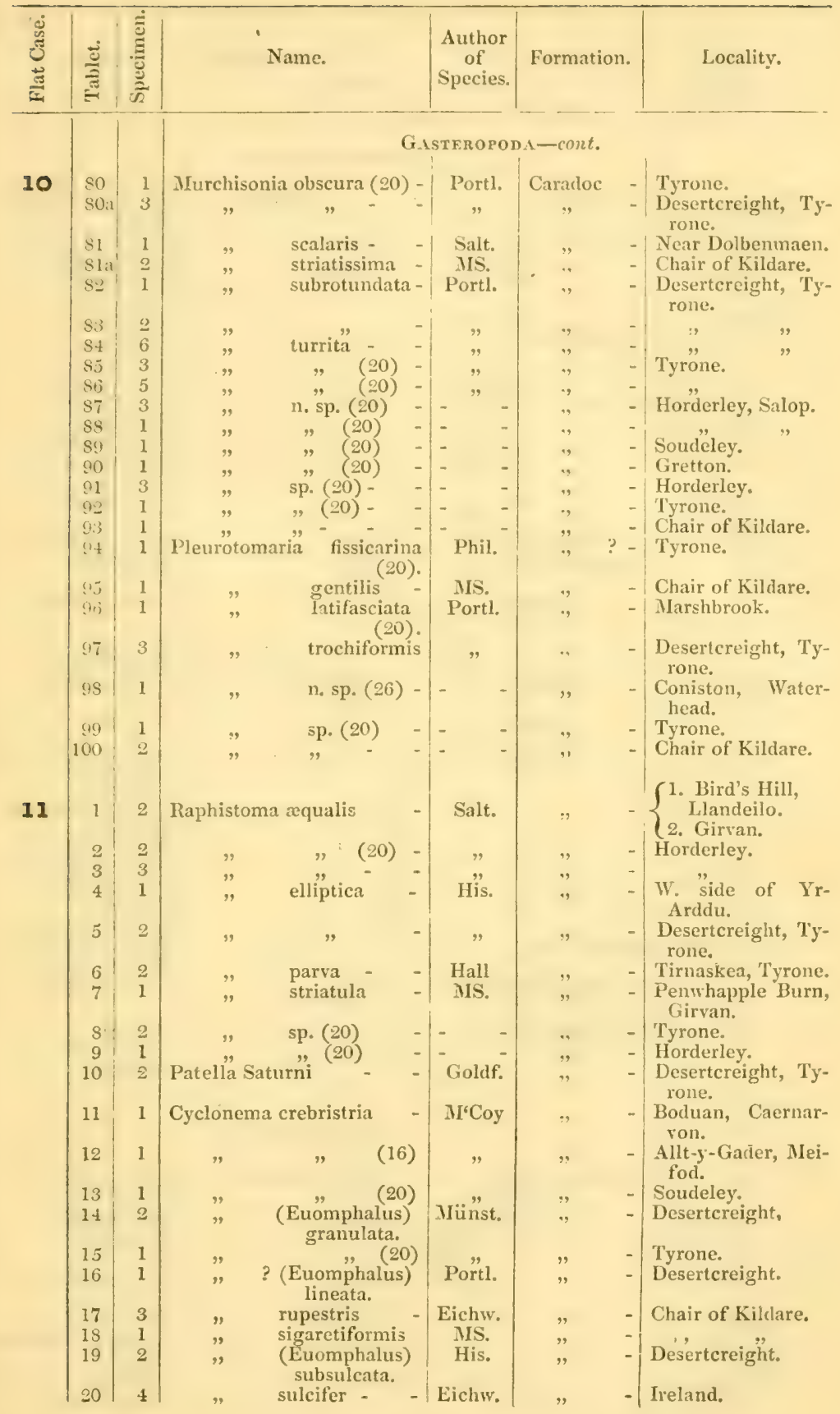



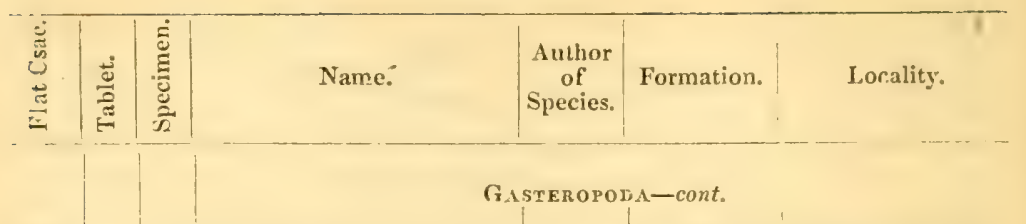

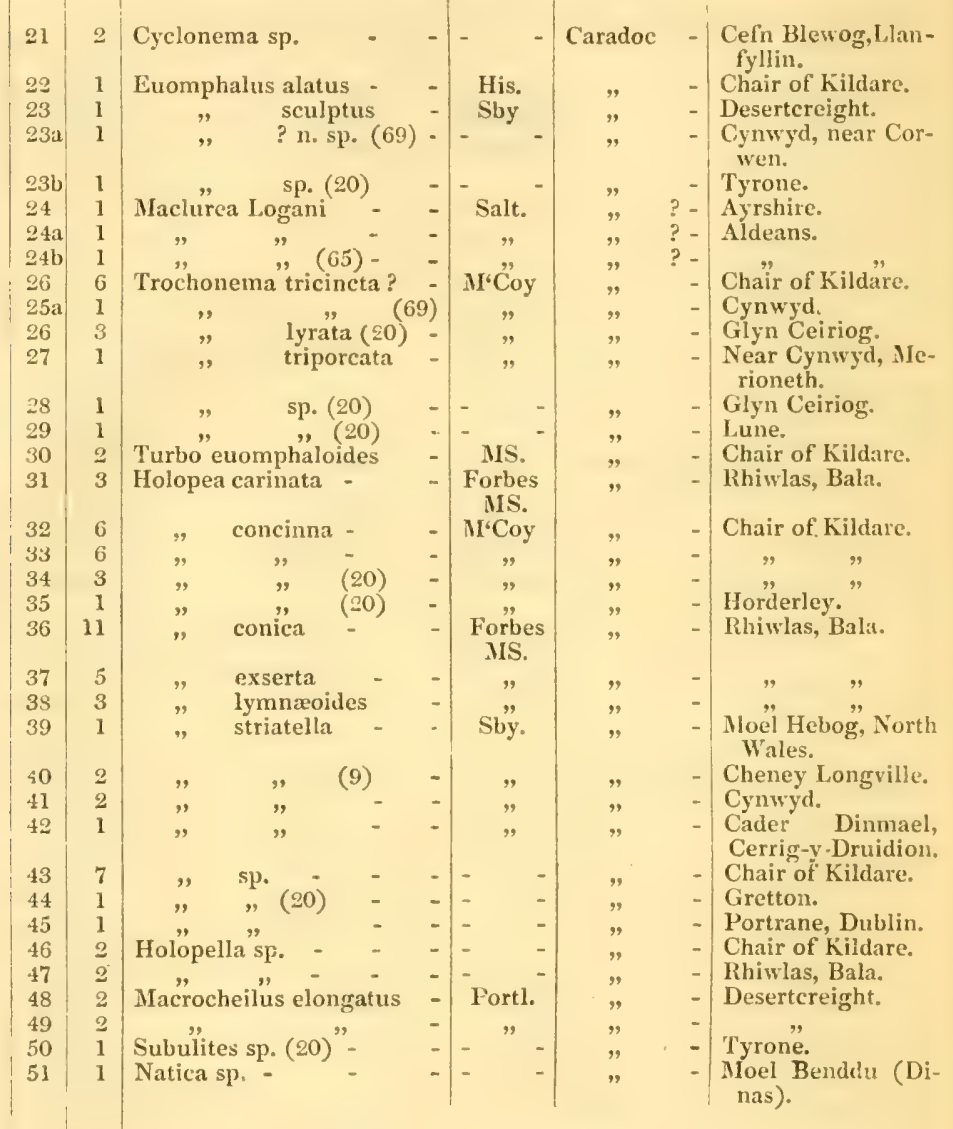

\section{PTEKOPODA.}

\begin{tabular}{|c|c|c|c|c|c|c|c|c|}
\hline 52 & 2 & Conularia & elongata & - & Portl. & Caradoc & - & Desertcreight. \\
\hline 53 & 2 & , & rectistriata & - & MIS. & , & - & Chair of Kildare. \\
\hline 54 & 1 & , & Sowerbyi & - & Defr & " & - & Desertcreight. \\
\hline 5 & 4 & , & , $\quad(20)$ & - & " & " & - & Acton Scott. \\
\hline 6 & 1 & $"$ & $(20)$ & - & " & " & - & C" \\
\hline & 1 & , & $(69)$ & - & " & " & - & $\begin{array}{l}\text { Cynwyd, near Cor- } \\
\text { wen. }\end{array}$ \\
\hline $\mathrm{za}$ & 2 & " & " & 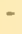 & $"$ & " & - & Cynwyd. \\
\hline & 1 & , & " & - & " & $"$ & - & $\begin{array}{l}\text { Gretton, } \\
\text { ton. }\end{array}$ \\
\hline & 1 & $"$ & n. sp. $(20)$ & - & - & $"$ & - & Horderley. \\
\hline & $!$ & $"$ & sp. $\quad-$ & - & - & ,. & - & Llwyn-yr-hweh. \\
\hline
\end{tabular}




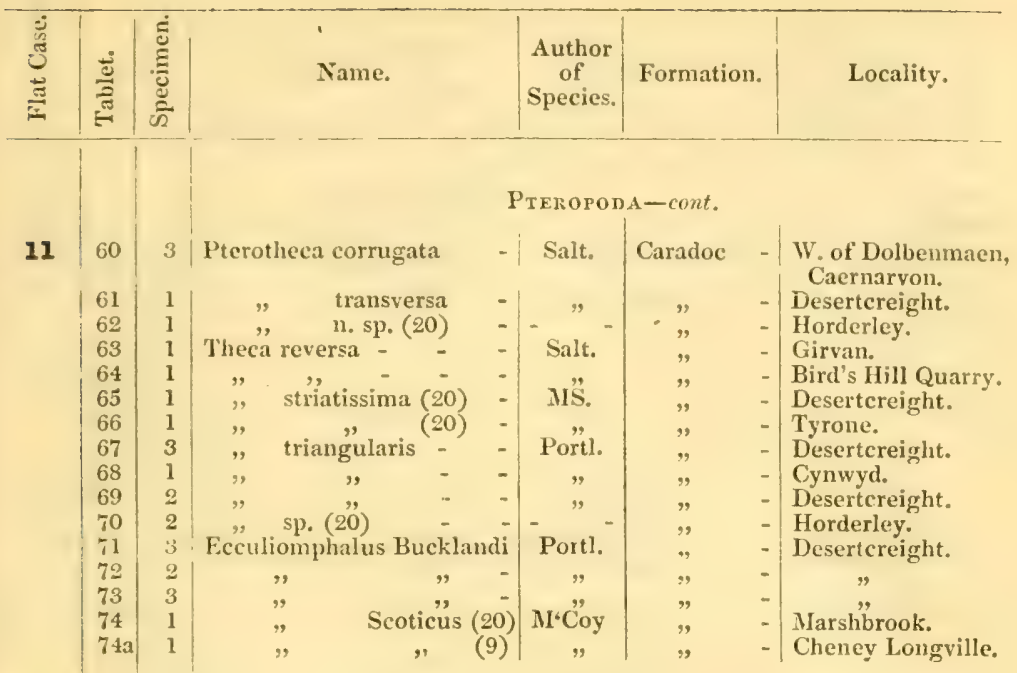

HETEROPODA.

\begin{tabular}{|c|c|c|c|c|c|c|c|c|}
\hline 75 & 4 & Bellerop & hon acutus & -1 & Sby. & Caradoc & - & Onny River and \\
\hline 76 & 1 & " & (20) & - & & $"$ & - & Tyrone. \\
\hline & 3 & ," & alatus - & - & Portl. & $"$ & -1 & Desertcreight. \\
\hline & 2 & ", & bilobatus & - & Sby. & $"$ & - & Hope Bowdler. \\
\hline & 3 & " & , & & " & " & -1 & $\mathrm{I}$ \\
\hline & & $"$ & (9) & & $"$ & $"$ & - & ey Longville \\
\hline & $\begin{array}{l}1 \\
5\end{array}$ & $"$ & $"$ & & $"$ & " & - & Corwen, \\
\hline & 5 & $"$ & $"$ & - & $"$ & $"$ & - & $\begin{array}{l}\text { Cynwyd. } \\
\text { Desertereigh }\end{array}$ \\
\hline & 1 & ", & (20) & & $"$ & $"$ & -1 & $\begin{array}{l}\text { Desertcreight. } \\
\text { Tyrone. }\end{array}$ \\
\hline & 1 & $m$ & (20) & & " & $"$ & 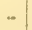 & \\
\hline & 3 & $"$ & (20) & & " & $"$ & -1 & Horderley. \\
\hline & 1 & " & (var. anceps) (20) & & $"$ & $"$ & - & Horderley, Salop. \\
\hline & 7 & ", & carinatus & - & " & $"$ & 1 & Twll-du. \\
\hline & 1 & $"$ & dilatatus & 1 & " & $"$ & - & $\begin{array}{l}\text { Desertcreight, ' } \\
\text { rone. }\end{array}$ \\
\hline 90 & 1 & $"$ & Griffithii (20) & & MS. & " & - & Soudeley. \\
\hline & 1 & $"$ & Ingualis (20) & & its & " & - & Tyrone. \\
\hline & 3 & $"$ & nodosus & & Salt. & " & -1 & $\begin{array}{l}\text { Hope Bowdler, } \\
\text { Church Stretton. }\end{array}$ \\
\hline & 1 & " & ", & - & " & " & -1 & Llwyn-yr-hwch. \\
\hline & 4 & " & $\Rightarrow \quad(20)$ & - & $"$ & $"$ & - & \\
\hline & 1 & , & " ? & - & " & " & 1 & Cynwyd, Merioneth. \\
\hline & 1 & $"$ & $"$ & & $"$ & ", & - & $\begin{array}{l}\text { Maen Bras, N. of } \\
\text { Bala. }\end{array}$ \\
\hline & 1 & ", & semirugosus & & Salt.MIS. & ", & - & Grweiddcau. \\
\hline & $\begin{array}{l}3 \\
0\end{array}$ & & ulcatin & & me & ", & - & of RalaLake \\
\hline & 1 & $"$ & surcannus & & 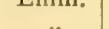 & $"$ & -7 & Onny River. \\
\hline & 1 & $"$ & n. sp." (20) & & - & " & & Onny River. \\
\hline & 2 & $"$ & $?$ & - & & ;, & - & Chair of Kildare. \\
\hline & 1 & $"$ & - & & - & " & - & Cefn Llwydlo. \\
\hline & 1 & " & - & & & $"$ & & $\begin{array}{l}\text { Bwlch - }- \text { Gase } \\
\text { Cynwyd. }\end{array}$ \\
\hline
\end{tabular}




\begin{tabular}{|c|c|c|c|c|c|c|}
\hline 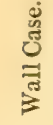 & 苛 & $\mid \begin{array}{c}\tilde{\Xi} \\
\stackrel{\Xi}{\Xi} \\
\text { हूँ } \\
\text { कू }\end{array}$ & Name. & $\begin{array}{l}\text { Author } \\
\text { of } \\
\text { Species. }\end{array}$ & Formation. & Locality. \\
\hline
\end{tabular}

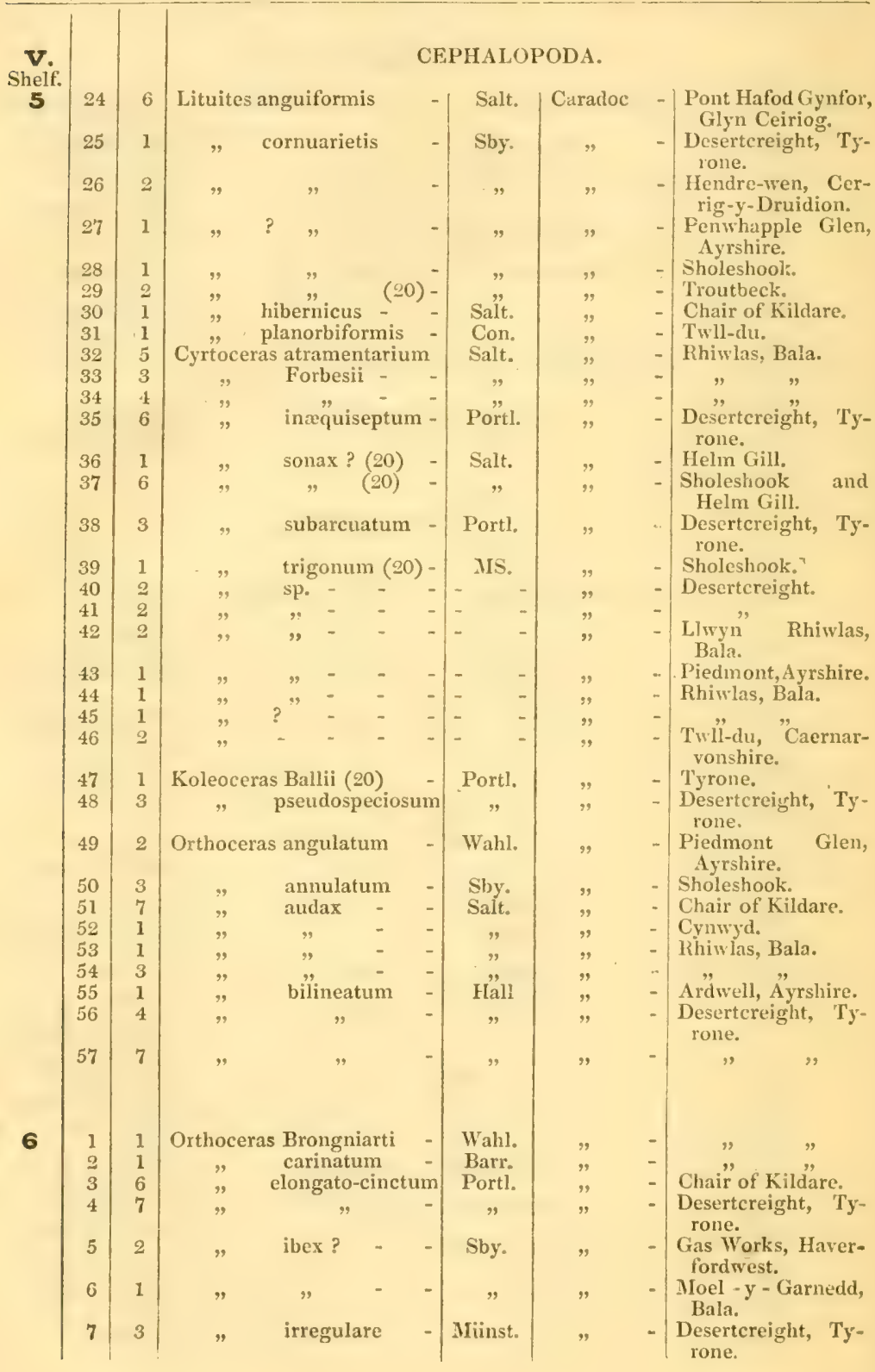




\begin{tabular}{|c|c|c|c|c|c|c|c|c|c|c|}
\hline \multirow{4}{*}{ 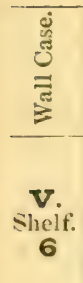 } & $\frac{3}{3}$ & 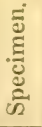 & \multicolumn{4}{|c|}{ Name. } & $\begin{array}{l}\text { Author } \\
\text { of } \\
\text { Species. }\end{array}$ & \multicolumn{2}{|c|}{ Formation. } & \multirow[t]{2}{*}{ Locality. } \\
\hline & & & \multicolumn{7}{|c|}{ CEPHALOTODA-cont. } & \\
\hline & 8 & 1 & Orthoceras $n$ & multianr & & & - & Caradoc & - & Wrexham. \\
\hline & 9 & 3 & , & perannu & latum & & Portl. & " & & $\begin{array}{l}\text { Desertcreight, Ty- } \\
\text { rone. }\end{array}$ \\
\hline & 10 & 4 & $"$ & politum & & - & $\mathrm{M} \cdot \mathrm{Coy}$ & . " & - & Ardwell, Ayrshire. \\
\hline & 11 & 1 & $"$ & $"$ & & - & $"$ & $=$ & - & $\begin{array}{l}\text { Penwhapple Glen, } \\
\text { Ayrshire. }\end{array}$ \\
\hline & 12 & 1 & $"$ & nor & & - & & " & - & De"rtcreioht" \\
\hline & 13 & 6 & $"$ & pomeroe & ense & - & Portl. & " & - & $\begin{array}{l}\text { Desertcreight, } \mathrm{Ty} \text { - } \\
\text { rone. }\end{array}$ \\
\hline & 14 & 1 & " & regulare & & - & Münst. & ", & - & Fermanagh. \\
\hline & 15 & 1 & $"$ & $"$ & & - & " & $"$ & - & $"$ \\
\hline & 16 & $\begin{array}{l}1 \\
2\end{array}$ & $"$ & recrulatu & ? & - & Salt."MS & $"$ & - & Chair" of Kildare. \\
\hline & is & $\overrightarrow{\mathrm{l}}$ & $"$ & subund & $\begin{array}{l}\text { ulatur } \\
? \text { ? }(2\end{array}$ & & Portl. & $"$ & - & Tyrone. \\
\hline & 19 & 5 & , & tenuicin & actum & & , & " & - & $\begin{array}{l}\text { Desertcreight, Ty- } \\
\text { rone. }\end{array}$ \\
\hline & 20 & 2 & ," & tenuistr & iatum & & Mïnst. & " & - & $" \quad "$ \\
\hline & 21 & 4 & ", & tumidun & & - & Portl. & $"$ & - & $" \quad " \quad "$ \\
\hline & 22 & 1 & $"$ & vagans & (20) & - & Salt. & $"$ & - & Bala. \\
\hline & $\begin{array}{l}23 \\
24\end{array}$ & $1 \frac{2}{17}$ & $"$ & " & $\begin{array}{l}- \\
-\end{array}$ & $\begin{array}{l}- \\
-\end{array}$ & $"$ & $"$ & - & Khiwlas, Bala. \\
\hline & 25 & 1 & $"$ & velatum & - & - & " - & $"$ & - & Chair of Kildare. \\
\hline & 20 & 1 & , & n. sp. & - & - & - - & $"$ & - & Ardwell, Ayrshire. \\
\hline & -7 & 1 & , & " & - & - & - & " & - & $\begin{array}{l}\frac{3}{4} \text { mile S. W. of Bryn } \\
\text { Bedwog,near Bala }\end{array}$ \\
\hline & 28 & 3 & , & " & - & - & - & " & - & $\begin{array}{l}\text { Myrddyn Morad, } \\
\text { Bala. }\end{array}$ \\
\hline & 29 & 1 & ," & sp. 2 & - & - & - & $"$ & - & Rhiwlas, Bala. \\
\hline & 30 & 1 & $"$ & $" 2$ & - & - & - & $"$ & - & $\begin{array}{l}\text { Sholeshook, Haver- } \\
\text { fordwest. }\end{array}$ \\
\hline & 31 & 4 & , &, 3 & - & - & - & ", & - & Rhiwlas, Bala. \\
\hline & 32 & 6 & ", & $" 4$ & - & - & - & ", & - & ., \\
\hline & 33 & 4 & " & " - & - & - & - & $"$ & - & $\begin{array}{l}\text { Bryn Bedwog, near } \\
\text { Bala. }\end{array}$ \\
\hline & 34 & 1 & ” & " - & - & - & - & " & - & Chair of Kildare. \\
\hline & 35 & 1 & $"$ & $"$ - & 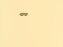 & - & - & $"$ & - & $\begin{array}{l}\text { Hendre-wen, Cer- } \\
\text { rig-y-Druidion, }\end{array}$ \\
\hline & 36 & 3 & " & , (20) & - & - & - & $"$ & - & Marshbrook and \\
\hline & 37 & 1 & " & ; (20) & - & -1 & - & $"$ & - & , \\
\hline & 38 & 1 & & " (20) & - & & - & & - & \\
\hline & 39 & 1 & ", & $"(20)$ & - & - & - & " & - & Soudeley. \\
\hline & 40 & 1 & " & $"(2 G)$ & - & - & & " & - & Troutbeck. \\
\hline & 41 & 3 & $\begin{array}{l}\text { Poterioceras } \\
\text { tum. }\end{array}$ & s appr & roxim & & $\mathbf{M I}^{\circ} \mathrm{Coy}$ & $"$ & - & $\begin{array}{l}\text { Desertcreight, } \mathrm{Ty} \text { - } \\
\text { rone. }\end{array}$ \\
\hline
\end{tabular}




\section{LOWER SILURIAN-cont.}

PIBEIT IEDS OF ZUDIFIG玨 SAITIRTON,

(Derived fossils).

\begin{tabular}{|c|c|c|c|c|c|c|}
\hline 产 & ब & 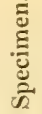 & Name. & $\begin{array}{l}\text { Author } \\
\text { of } \\
\text { Species. }\end{array}$ & Formation. & Locality. \\
\hline
\end{tabular}

\section{ECHINODERMATA.}

Cyathocrinus? (72) - - i - $\quad$ - Pebble Beds ; Budleigh Salterton.

\section{ANNELIDA.}

\section{CRUSTACEA.}

3 - 2 Phacops incertus (72) - Deslong. Pebble Beds | Budleigh Salterton.

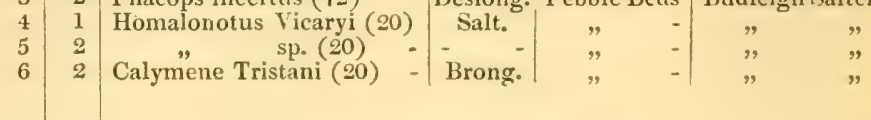

\section{BRACHIOPODA.}

\begin{tabular}{llll|l|l|l}
7 & I & Spirifera antiquissima (72) \\
8 & 1 & $y$ & MS. & Pebble Beds & Budleigh Salterton.
\end{tabular}

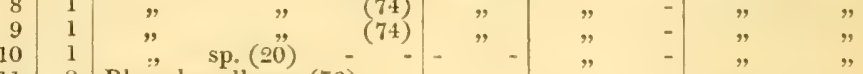

\begin{tabular}{ll|l}
10 & 1 & sp. $(20)$ \\
11 & 2 & Rhynchonella sp. (79) \\
12 & 2 &
\end{tabular}

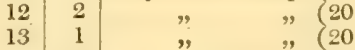

14

15

Orthis pulvinata (20)

$" \quad " \quad(74)$

17

18

19

20

9

$"$ redux (20) (var.Budleighensis, Salt.)

$21 \quad 1 \mid \quad, \quad, \quad(75)$

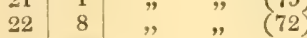

239 9,

$\begin{array}{lllll}24 & 3 & \text {, } & \end{array}$

\begin{tabular}{l|l|ll}
25 & 1 &, & " \\
26 & 1 & , & n. sp. (20)
\end{tabular}

Strophomena sp. (20)

Strophalosia sp. (20)

Discina n. sp. (20) -

Lingula crumena (74)

Hawkeii (74)

Lesueuri (7t)

",
"
"
, -
-
-
-
-
-

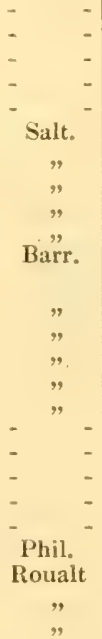

$"$
$"$
$"$
$"$
$"$
$"$
$"$
$"$
$"$
$"$
$"$
$"$
$"$
$"$
$"$
$"$
$"$
$"$ 
LOWER SILURIAN-cont.

LOWER IIANDOVIRX.

\begin{tabular}{|c|c|c|c|c|c|c|c|}
\hline 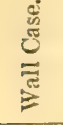 & 造 & हूँ & Name. & & $\begin{array}{l}\text { Author } \\
\text { of } \\
\text { Species. }\end{array}$ & Formation. & Locality. \\
\hline & & & \multicolumn{5}{|c|}{ VARIOUS LARGE SPECIMENS. } \\
\hline ielf. & 13 & I & $\begin{array}{l}\text { Atrypa hemispherica } \\
\text { Orthis reversa - - } \\
\text { Meristella angustifrons } \\
\text { Petraia subduplicata - }\end{array}$ & $\begin{array}{l}- \\
- \\
-\end{array}$ & $\left.\begin{array}{c}\text { Sby. } \\
\text { Salt. } \\
M^{\prime} \mathrm{Coy} \\
, y\end{array}\right\}$ & $\begin{array}{c}\text { Lower } \\
\text { Llandovery. }\end{array}$ & $\begin{array}{l}\text { Mullock's Hill, Dal- } \\
\text { guharran. }\end{array}$ \\
\hline & 14 & 1 & $" \quad \quad " \quad-$ & & $"$ & , & $\begin{array}{l}\text { Priory Hill, Haver- } \\
\text { fordwest. }\end{array}$ \\
\hline & 15 & 1 & Meristella angustifrons & - & " & " & $\begin{array}{l}\text { Mullock's Hill } \\
\text { Quarry, Ayrshire. }\end{array}$ \\
\hline & 16 & 1 & $\begin{array}{l}\text { Pleurotomaria Moorei } \\
\text { Murchisonia cancellata }\end{array}$ & 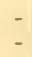 & $\left.\begin{array}{c}\text { Salt. } \\
\text { M'Coy }^{\prime}\end{array}\right\}$ & $"$ & $\begin{array}{l}\text { Thrave, Girvan, } \\
\text { Ayrshire. }\end{array}$ \\
\hline & 17 & 1 & Annelida - - - & - & $-\quad-$ & " & $\begin{array}{l}\text { Nant-y-carfan, } \mathrm{S} . \\
\text { Wales. }\end{array}$ \\
\hline & 18 & 1 & Periechocrinus - & - & - & $"$ & Great Barr, Walsall. \\
\hline & 13 & 1 & Annelide burrows & 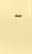 & - & $"$ & $\begin{array}{l}\text { Pant-y-dur, } 4 \mathrm{~m} \text {. } \\
\text { N.E. of Liandeilo. }\end{array}$ \\
\hline & 20 & 1 & , & - & - & . & $"$ \\
\hline & 21 & 1 & " & 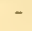 & - & " & $"$ \\
\hline
\end{tabular}

PLANTE.

Fucoids? - $\quad-\quad-\quad-|-\quad-| \begin{gathered}\text { Lower } \\ \text { Llandovery. }\end{gathered} \mid$ Hop Mill, Shelve.

SPONGIDA.

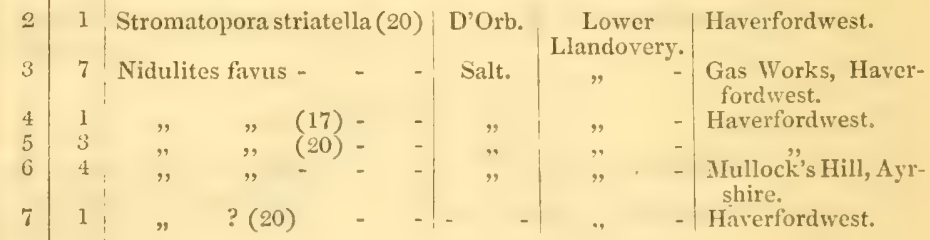

ACTINOZOA.

\begin{tabular}{|c|c|c|c|c|c|c|c|}
\hline 8 & 2 & \multicolumn{2}{|l|}{ Heliolites interstincta } & Wahl. & \multicolumn{2}{|c|}{$\begin{array}{c}\text { Lower } \\
\text { Llandovery. }\end{array}$} & $\begin{array}{l}\text { Gas Works, Haver- } \\
\text { fordwest. }\end{array}$ \\
\hline 9 & 1 & " & $(20)$ & ", & , & - & Haverfordwest. \\
\hline 10 & 1 & ", & 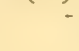 & $"$ & $"$ & - & $\begin{array}{l}\text { Mandinam, Llan- } \\
\text { dovery. }\end{array}$ \\
\hline 11 & 1 & $"$ & - & $"$ & ", & - & $\begin{array}{l}\text { Priory Mill, Haver- } \\
\text { fordwest. }\end{array}$ \\
\hline 12 & 1 & tubulata & - & Lonsd. & , & - & Cefn-y-garreg. \\
\hline 1 & 2 & Alveolites La Bechii & - & M. Edw. & " & - & Cong, co. Galway. \\
\hline $1+$ & 1 & Favosites aspera & - & $D^{\prime} O r b$. & " & - & $\begin{array}{l}\text { W. side of Cefin-y- } \\
\text { garreg, Llando- } \\
\text { very. }\end{array}$ \\
\hline
\end{tabular}




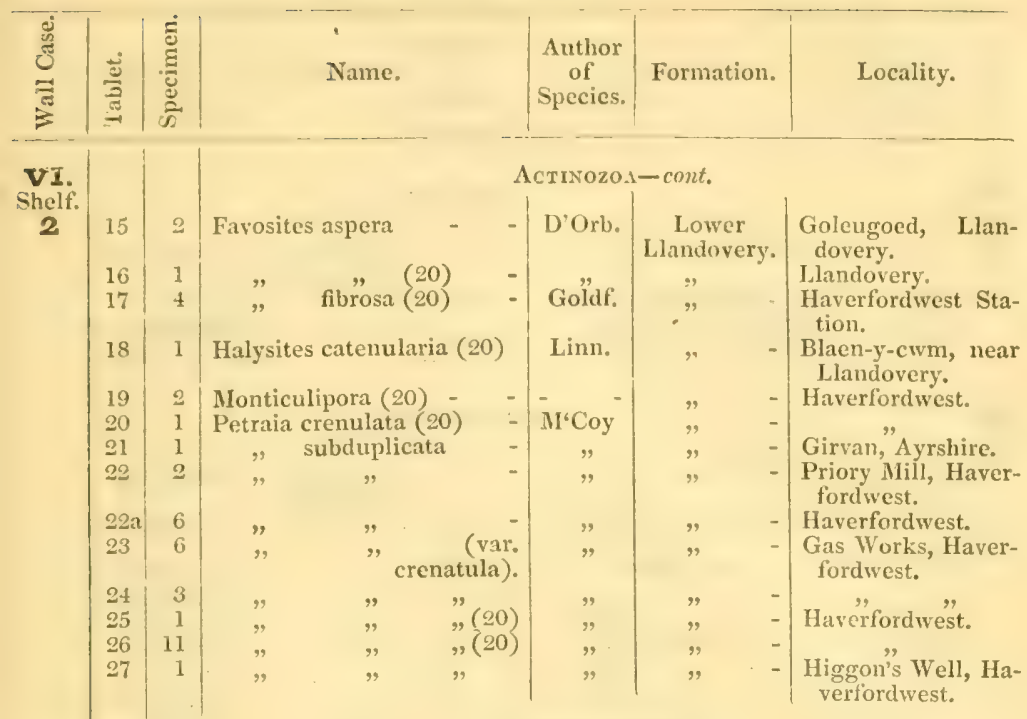

\section{ECHINODERMATA.}

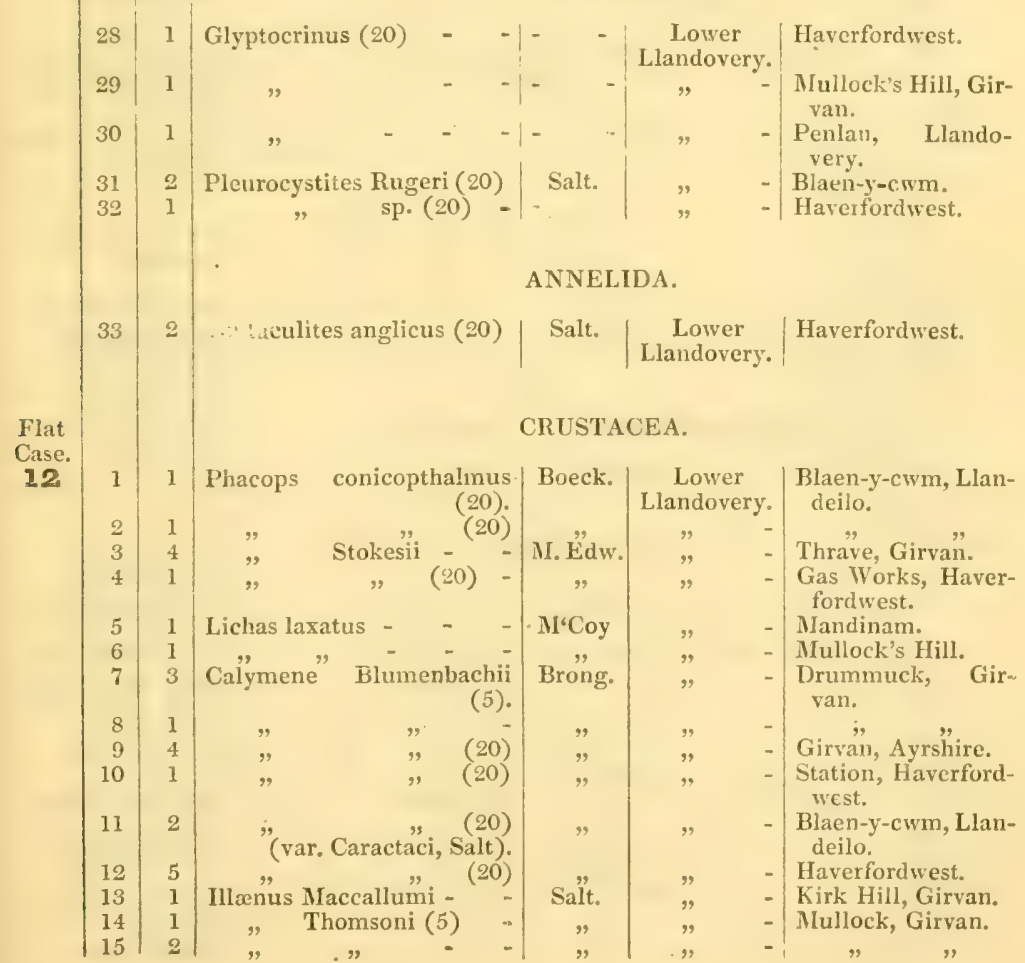




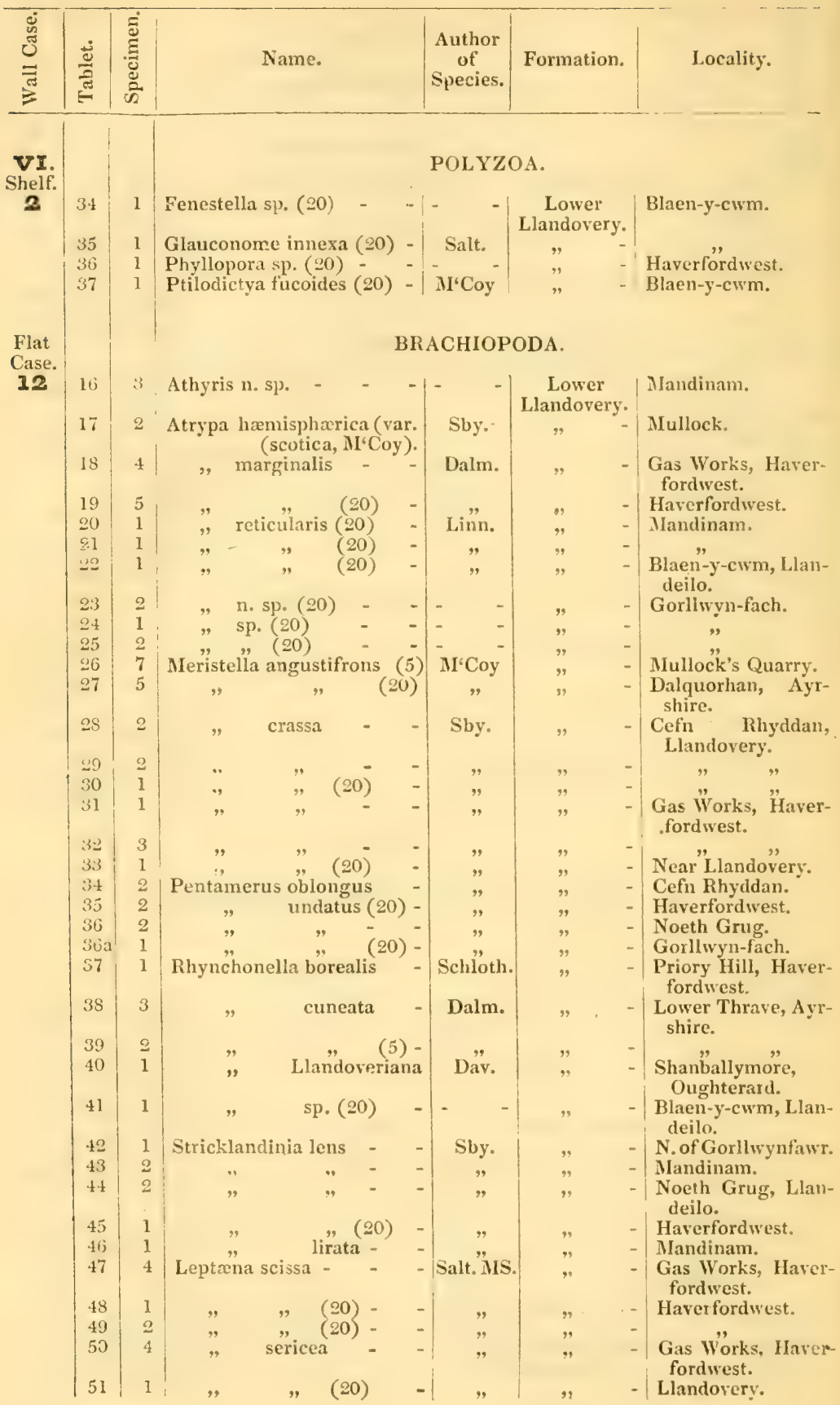




\begin{tabular}{|c|c|c|c|c|c|c|}
\hline 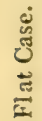 & $\frac{\stackrel{d}{2}}{\rightleftarrows}$ & 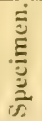 & Name. & $\begin{array}{c}\text { Author } \\
\text { of } \\
\text { species. }\end{array}$ & Formation. & Locality. \\
\hline
\end{tabular}

Brachorona-cont.

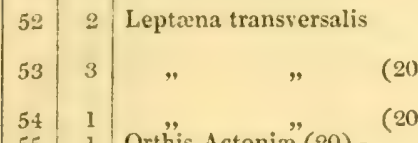

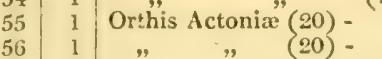

$$
" \text { Bouchardii - }
$$$$
\because \quad \text { ca }
$$

Wahl,

rhomboidalis Winn.

".

" Lower
Llandovery. Blaen-y-cwm, Llandeilo.

Haverfordwest. "

Cefn Lïwydlo.

N. of Cilgwyn.

Llandovery."

Blaen-y-cwm, Llandeilo.

Cong, Galway.

Mullock's Hill.

Llandovery.

Blaen-y-cwm, Llan deilo.

Mandinam.

- Gorllwyn-fach.

- Blaen-y-cwm, Llandeilo.

- Penlä Qüarry, Llandovery.

- Mandinam.

- Mullock, Girvan.

- " " "

? Gas Works, Haverfordwest.

- Blaen-y-cwm, Llandeilo.

? Gas Works, Haverfordwest.

- Haverfordwest.

- Cefn Rhyddan.

- Blaen-y-civm.

? Gas Works, Haverfordwest.

- Blaen-y-cwm, Llandeilo.

Haverfordwest.

Cong, G̈alway.

Haverfordwest.

S. Wales (Havetfordwest?).

Blaen-y-cwm, Llandeilo. 


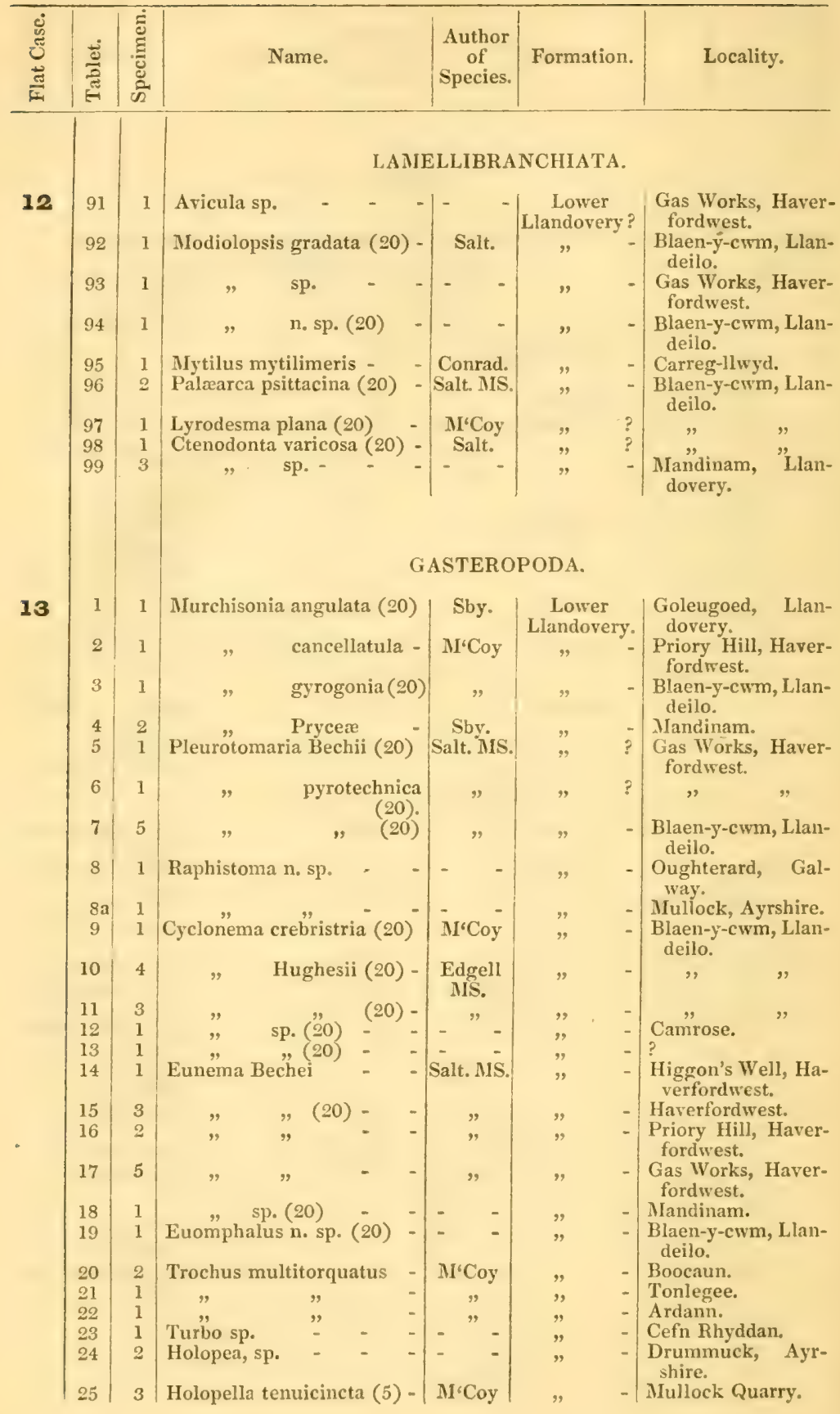




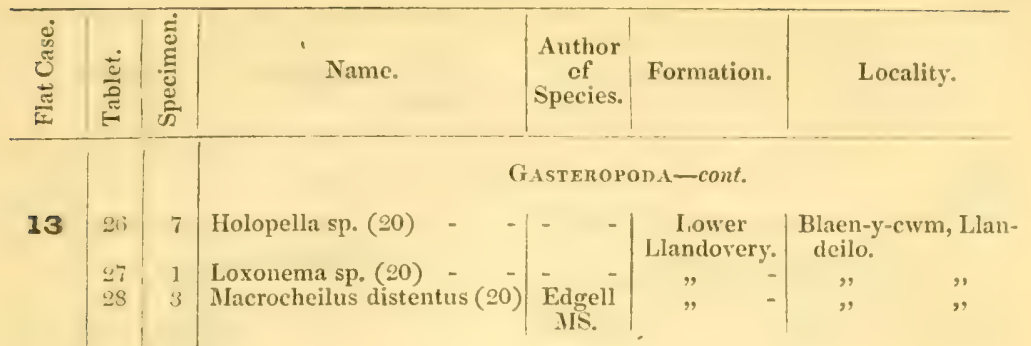

\section{PTEROPODA.}

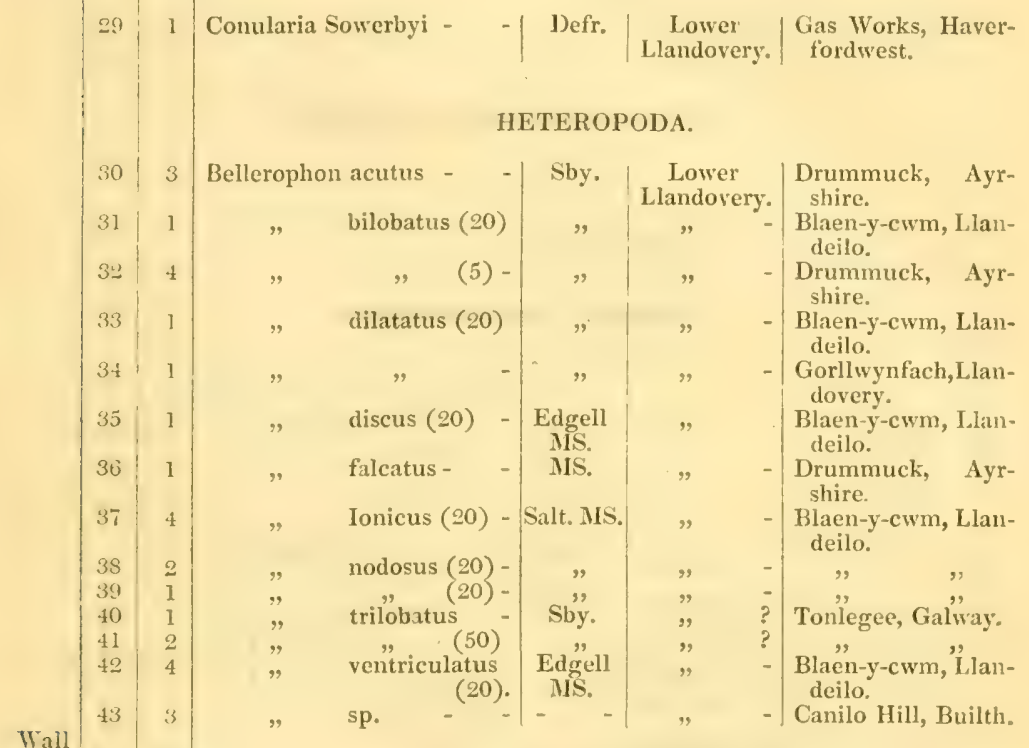

CEPHALOPODA.

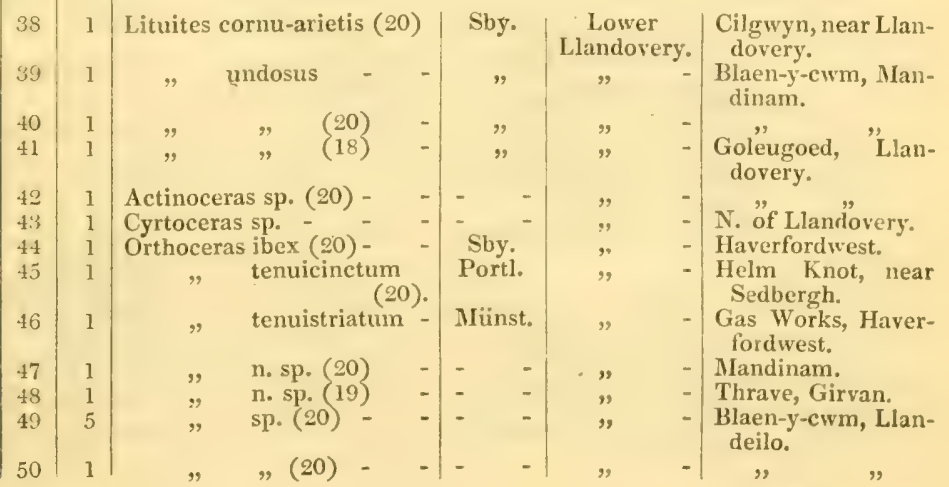




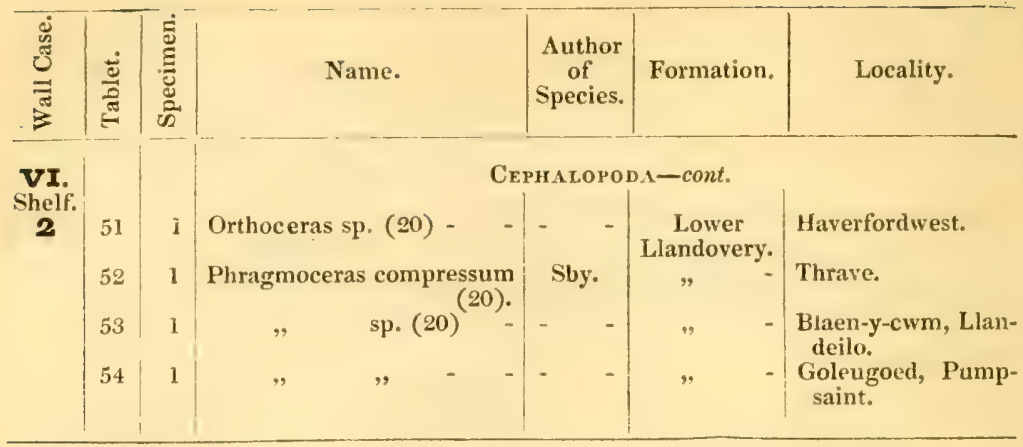

LOWER PALEOZOIC SERIES.

UPPER SILURIAN.

UPPIR IIANDOVERY.

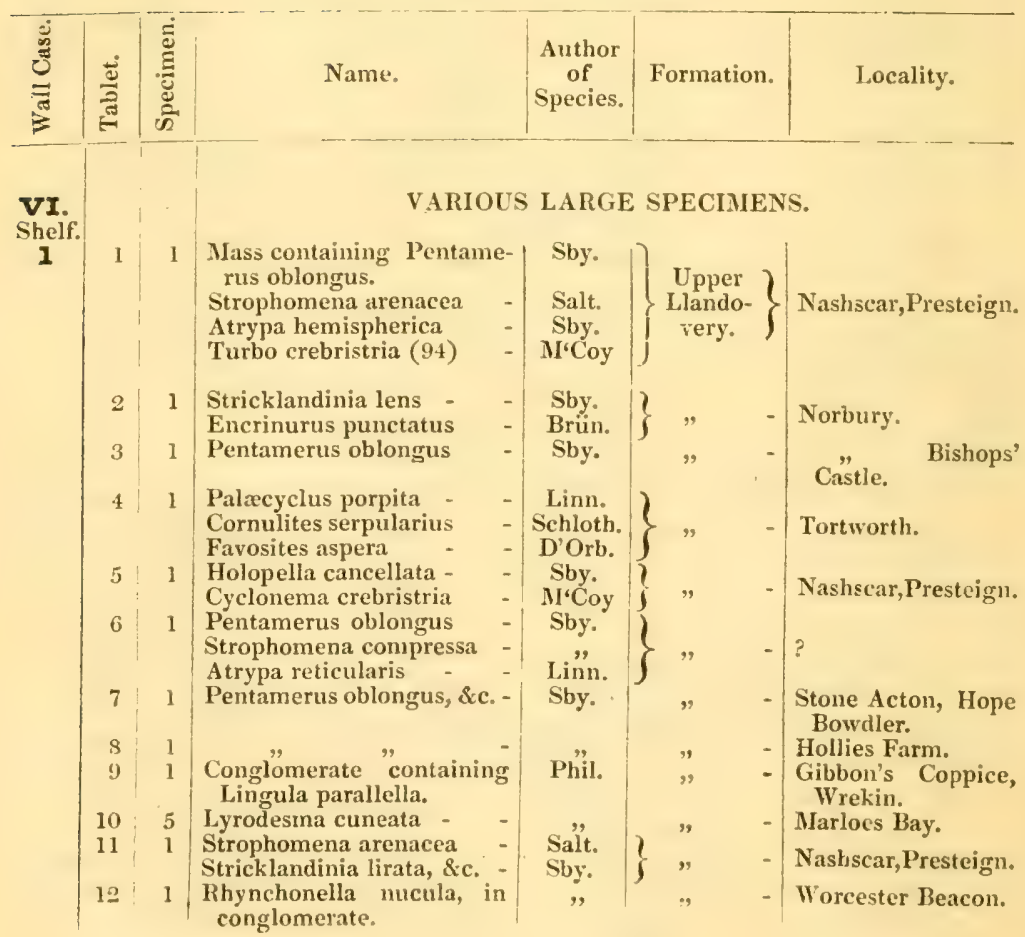




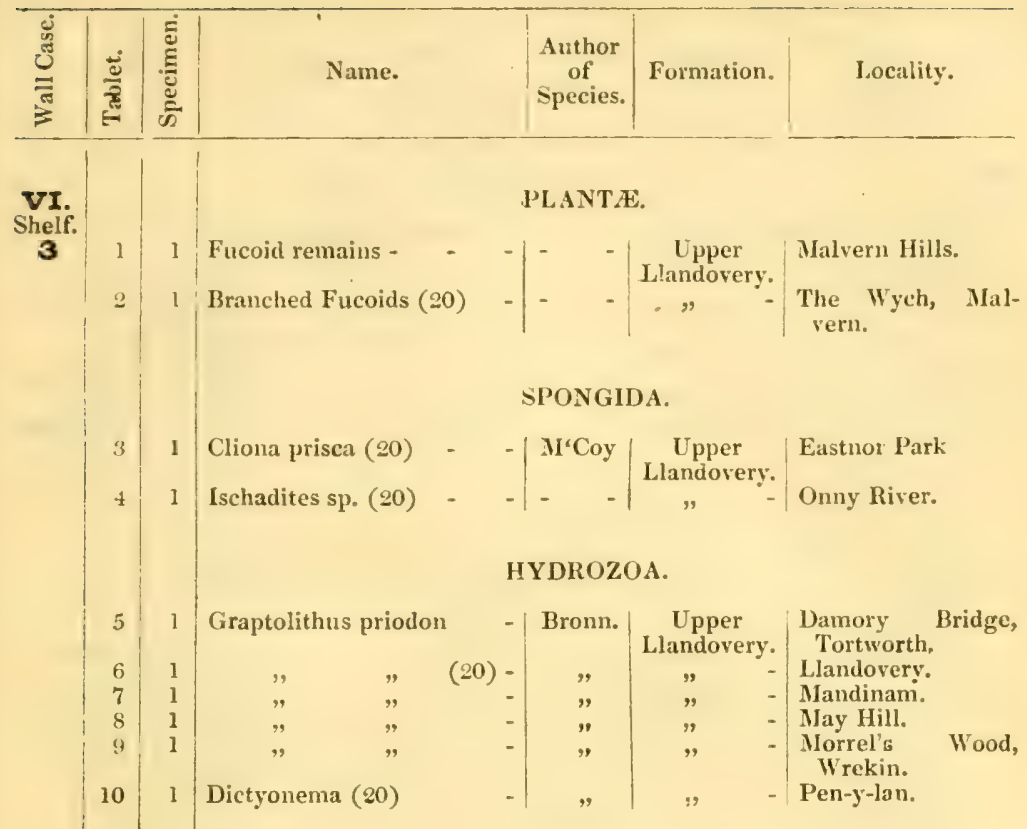

\section{ACTINOZOA.}

\begin{tabular}{|c|c|c|c|c|c|c|}
\hline 11 & 1 & Heliolites farosa (9) & - & - $\mathrm{MI}^{\prime} \mathrm{Coy}$ & $\begin{array}{l}\text { Upper } \\
\text { I.landovery }\end{array}$ & Hope Quarry. \\
\hline 12 & 1 & inordinata & $1-$ & Lonsd. & , & - Presteign. \\
\hline & 1 & interstincta & a & Wahl. & $"$ & - Huntley Hill. \\
\hline & 1 & Favosites aspera & - & $\mathbf{M}^{6} \mathrm{Coy}$ & " & $\begin{array}{l}\text { Damory Bridge, } \\
\text { 'Tortworth. }\end{array}$ \\
\hline & 1 & ", & - & " & $"$ & $\begin{array}{l}\text { - Hope Quarry, Min- } \\
\text { sterly. }\end{array}$ \\
\hline & 1 & " & 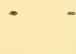 & $"$ & $"$ & $\begin{array}{l}\text { W. side of Cefn-y- } \\
\text { garreg. }\end{array}$ \\
\hline & 2 & fibrosa & - & Goldf. & $"$ & - Huntley Hill. \\
\hline & & , (20) & - & $"$ & $"$, & - Onny Ŕiver. \\
\hline & 1 & Gothlandica & $a(20)$ & Fougt. & $"$ & \\
\hline & 1 & $"$ & $(20)$ & " & " & $\begin{array}{l}\text { - Saugh Hill, Gir- } \\
\text { van. }\end{array}$ \\
\hline & 2 & multipora & - & Lonsd. & $"$ & - $\begin{array}{l}\text { Howlers Heath, } \\
\text { Malvern. }\end{array}$ \\
\hline & 1 & " " & - & & $"$ & - Bog Mine, Shelve. \\
\hline & 1 & Halysites catenularia & 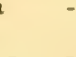 & Linn. & $"$ & - Castell craiggwydd- \\
\hline & l & " & - & $"$ & $"$ & - $\begin{array}{c}\text { Penlan, Llando- } \\
\text { very. }\end{array}$ \\
\hline & 1. & Syringopora serpens & - & " & "’ & - Norbury. \\
\hline & 1 & sp. (20) & - & - & " & - $\begin{array}{l}\text { The Wych, Mal- } \\
\text { vern. }\end{array}$ \\
\hline & 2 & Cyathophyllum sp. & - & - & " & - Bog Mine, Shelve. \\
\hline & 1 & Omphyma sp. - & - & & $"$ & w \\
\hline & 1 & Palxocyclus porpita & - & Linn. & " & $\begin{array}{l}\text { W. of Crockleys, } \\
\text { near Tortworth. }\end{array}$ \\
\hline & 3 & 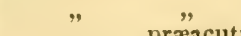 & - & & $"$ & Huntley Hill. \\
\hline & & ", & & two. & $"$ & May Hill. \\
\hline & 1 & $"$ & & $"$ & $"$ & " \\
\hline
\end{tabular}




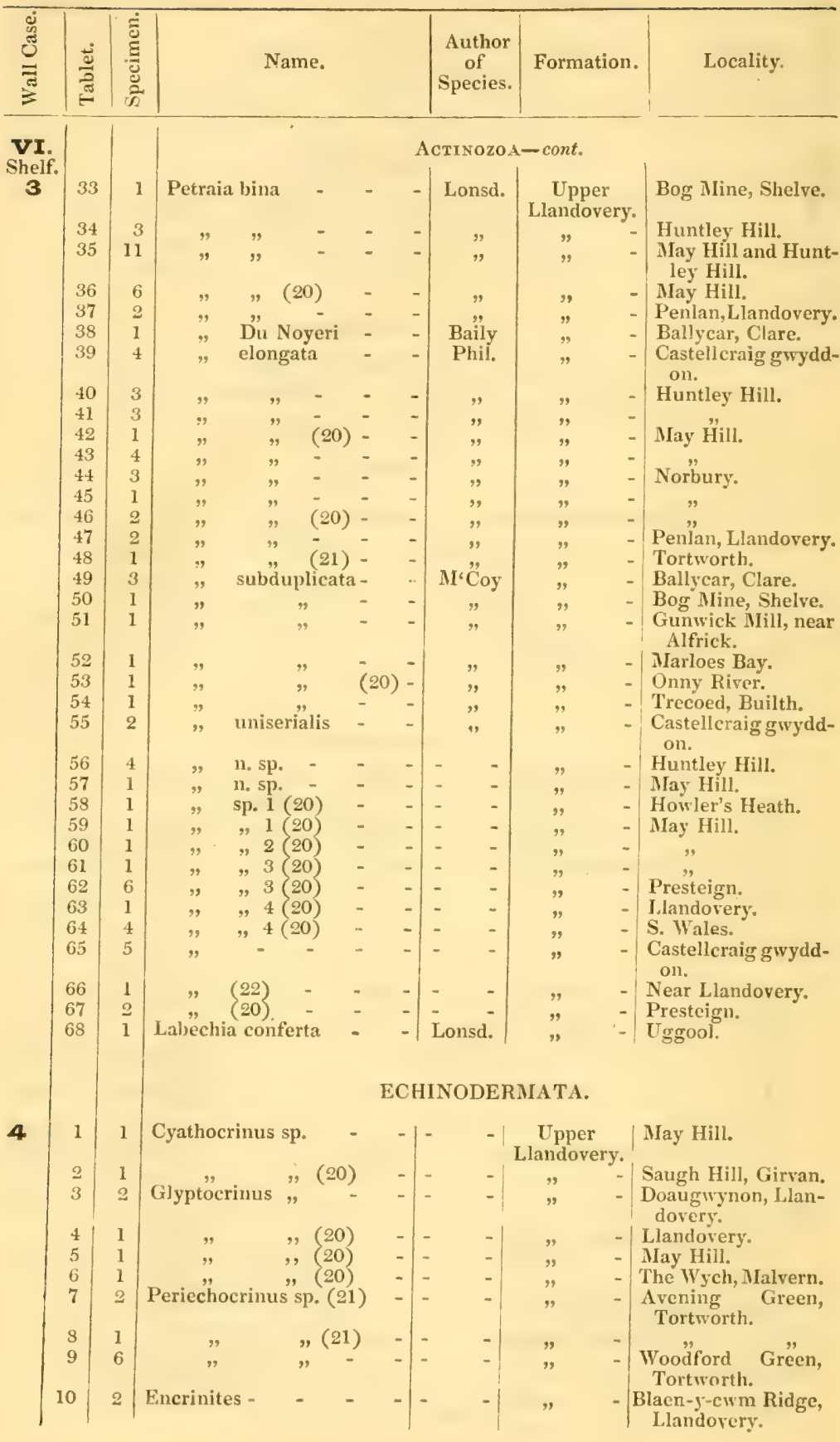




\begin{tabular}{|c|c|c|c|c|c|c|}
\hline 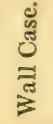 & है & $\begin{array}{l}\text { हूं } \\
\text { हूँ } \\
\text { कू } \\
\text { का }\end{array}$ & Name. & $\begin{array}{l}\text { Author } \\
\text { of } \\
\text { Species. }\end{array}$ & Formation. & Locality. \\
\hline
\end{tabular}

VI.

Shelf.

4

Fla

13

Echinodermata-cont.

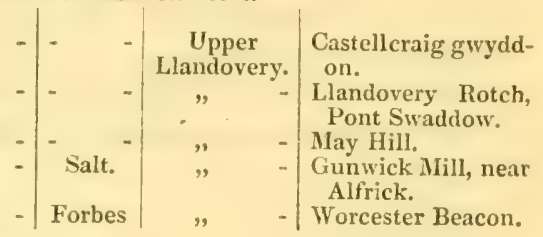

\section{ANNELIDA.}

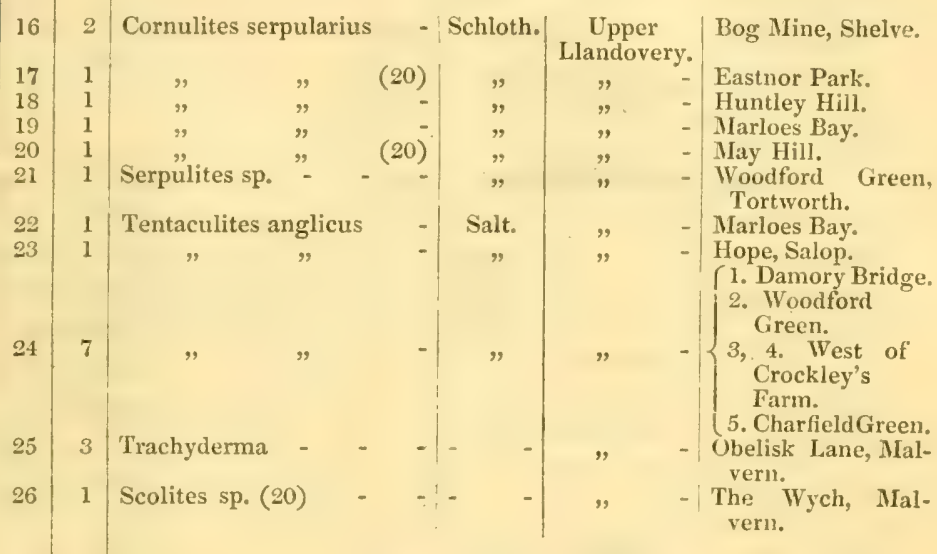

\section{CRUSTACEA.}

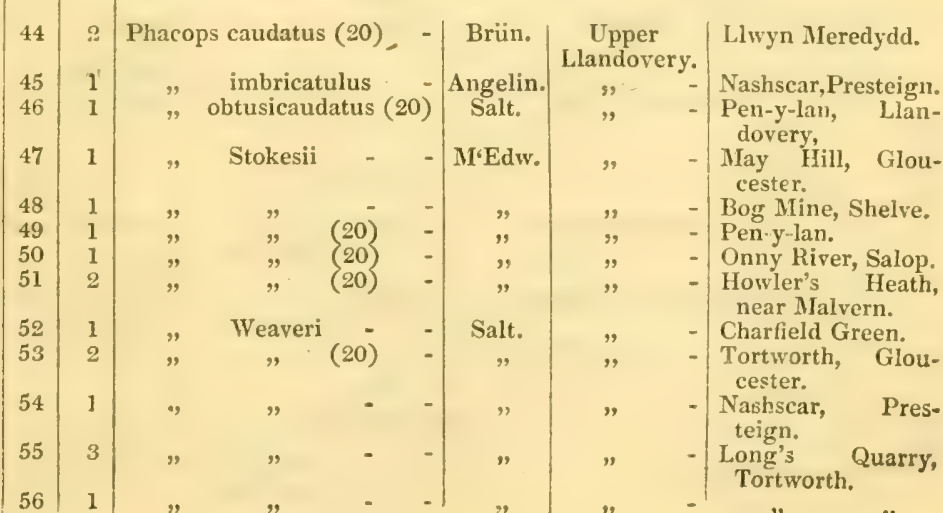




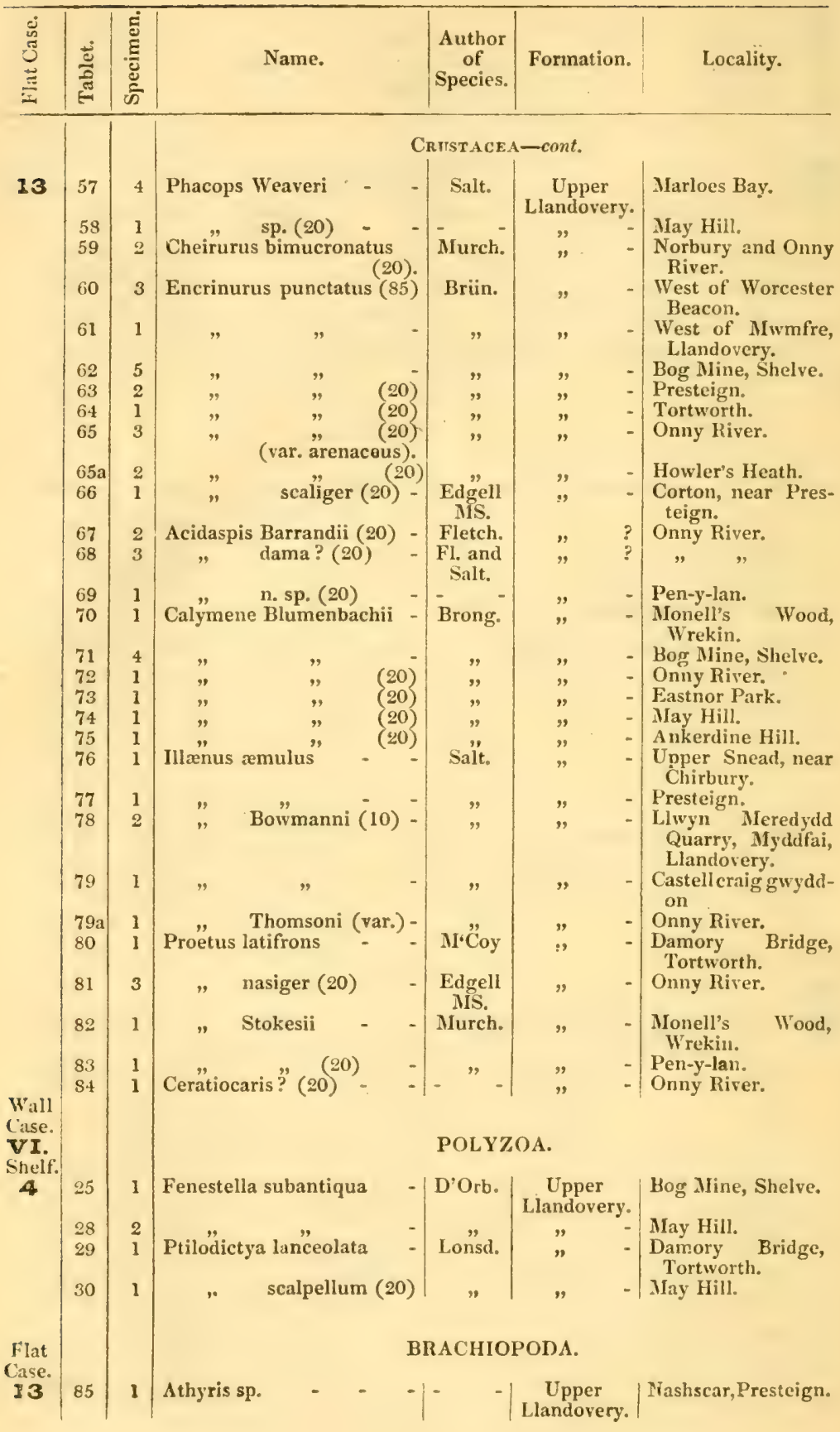




\begin{tabular}{|c|c|c|c|c|c|c|}
\hline 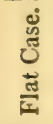 & $\frac{\vec{\omega}}{\mathrm{d}}$ & कू & Name. & $\begin{array}{l}\text { Author } \\
\text { of } \\
\text { Species. }\end{array}$ & Fornuation. & Locality. \\
\hline
\end{tabular}

\section{BRACHOPODA-cont.}

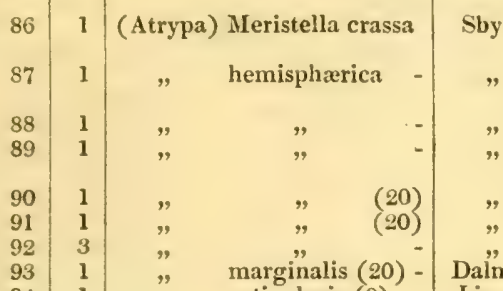$$
\text { (20) - }
$$

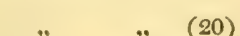

(20)

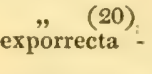

\section{Upper | Llandovery.} Llandovery.

\begin{tabular}{|c|c|c|}
\hline " & - & Charfield Green, \\
\hline & & Acton Scott. \\
\hline$"$ & & $\begin{array}{l}\text { Monell's Wood, } \\
\text { Wrekin. }\end{array}$ \\
\hline , & & Ankerdine Hill. \\
\hline " & & Presteign. \\
\hline " & & Marloes Bay. \\
\hline " & & Mandinam. \\
\hline " & & Hope Quarry. \\
\hline 3 & & Huntley Hill. \\
\hline
\end{tabular}

Shropshire.

- Norbury.

- Presteign.

- Woodford Green, Tortworth.

- May Hill.

- Mandinam.

- Gorllwyn-fach.

Presteign.

- May Hill.

- Huntley Hill.

- Pen-y-lan.

- Damory Bridece, Toriworth.

May Hill.

Damory Bridge, Tortworth.

- Hope Quarry, Chirbury.

- The Hollies, Wrekin.

- Nashscar,Presteign.

- Norbury, Shropshire.

- Craig - yr - wyddon, Llandovery.

- Norbury.

- Woodford Hill, Tortworth

- Presteign.

- Norbury.

- Llwynwormwood.

- Penlan.

- Pen-y-lan.

- Nashscar.

- Llandovery.

- Obelisk, Eastnor Park.

- Presteign. 


\begin{tabular}{|c|c|c|c|c|c|c|}
\hline 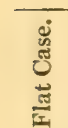 & 总 & 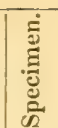 & Name. & $\begin{array}{l}\text { Author } \\
\text { of } \\
\text { Species. }\end{array}$ & Formation. & Locality. \\
\hline
\end{tabular}

\section{Brachiopoda-cont.}

\begin{tabular}{|c|c|c|c|c|c|}
\hline 18 & 1 & Rhynchonella decemplicata & Sby. & Upper & Pen-y-lan. \\
\hline & 3 & $\begin{array}{r}\text { Llandoveriana } \\
(20) \text {. }\end{array}$ & Dav。 & $"$ & Cappacorcoga. \\
\hline & 2 & nucula & Sby. & $"$ & Marloes Bay. \\
\hline & $\begin{array}{l}1 \\
4\end{array}$ & Wilsoni $^{(20)}$ & $"$ & $"$ & $\begin{array}{l}\text { Landovery, } \\
\text { Huntley Hill, May } \\
\text { Hill. }\end{array}$ \\
\hline
\end{tabular}

\section{Stricklandinia}

"

Sby.

$$
\begin{aligned}
& \begin{array}{l}
- \\
- \\
-
\end{array} \\
& \text {," } \\
& \text { - " }
\end{aligned}
$$$$
\text { , }
$$$$
9
$$$$
\text { - }
$$$$
\text { salt. MIS. }
$$ 


\begin{tabular}{|c|c|c|c|c|c|c|}
\hline 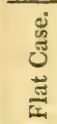 & हैं & 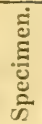 & Name. & $\begin{array}{l}\text { Author } \\
\text { of } \\
\text { Species. }\end{array}$ & Formation. & Locality. \\
\hline
\end{tabular}

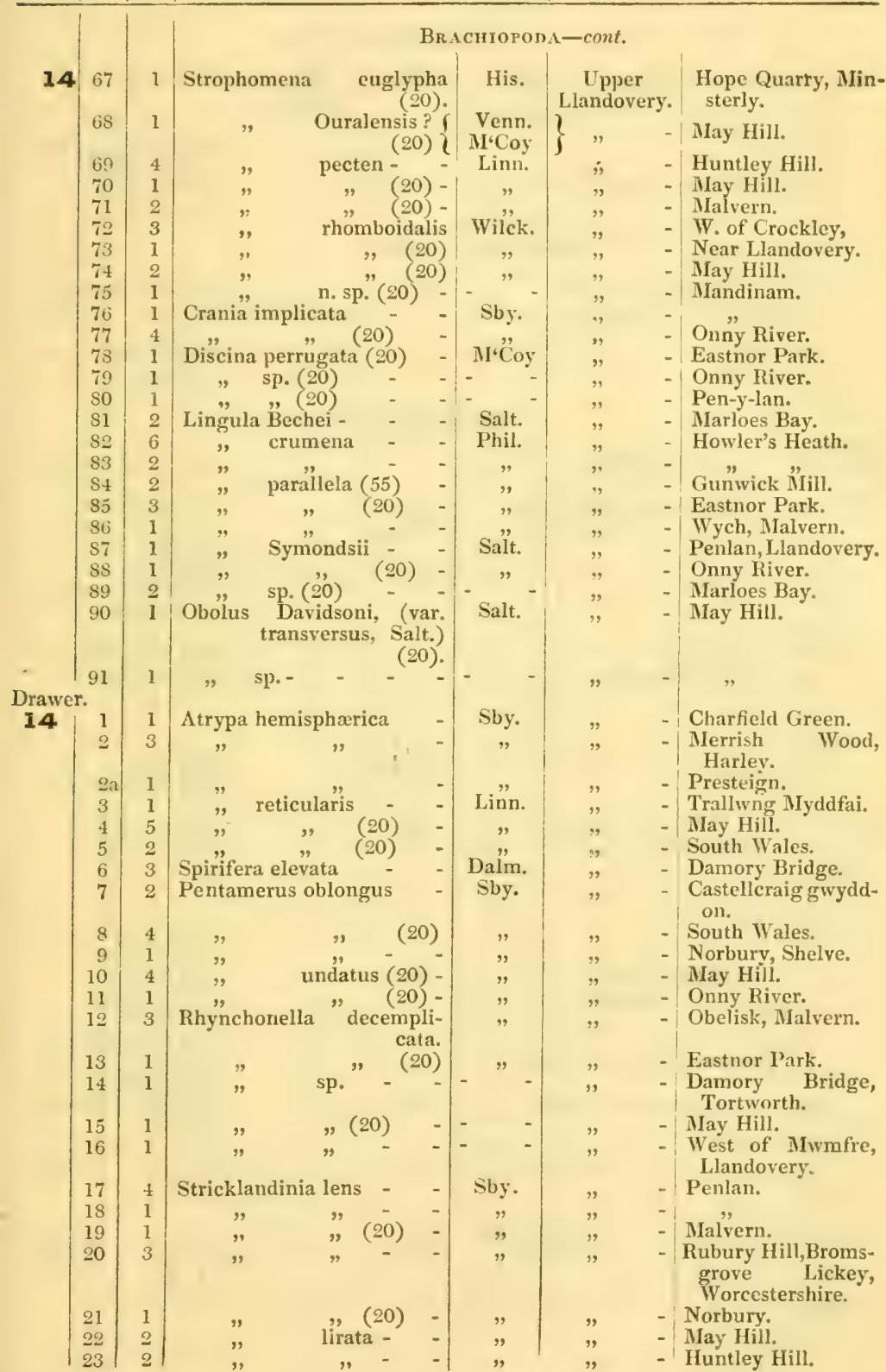




\begin{tabular}{|c|c|c|c|c|c|c|}
\hline 离 & 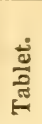 & 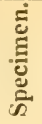 & Name. & $\begin{array}{l}\text { Author } \\
\text { of } \\
\text { Species. }\end{array}$ & Formation. & Locality. \\
\hline
\end{tabular}

BRACHIOPODA-cont.

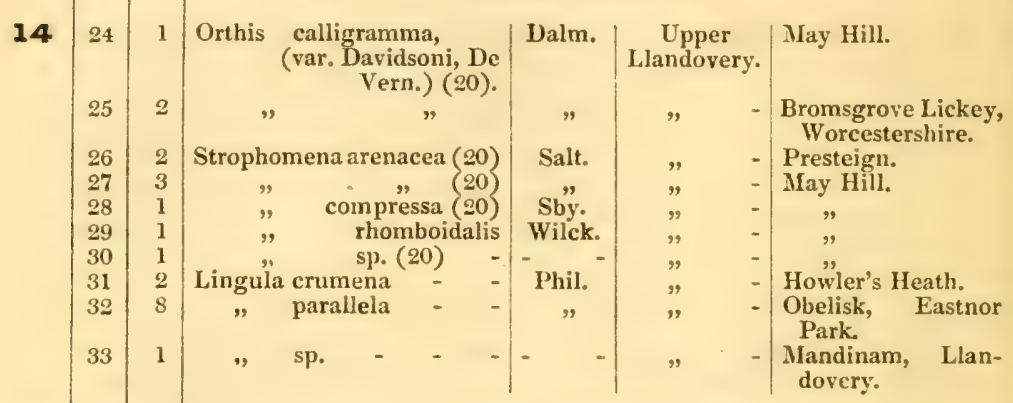

Flat

Case.

14

15

\section{LAMELLIBRANCHIATA.}

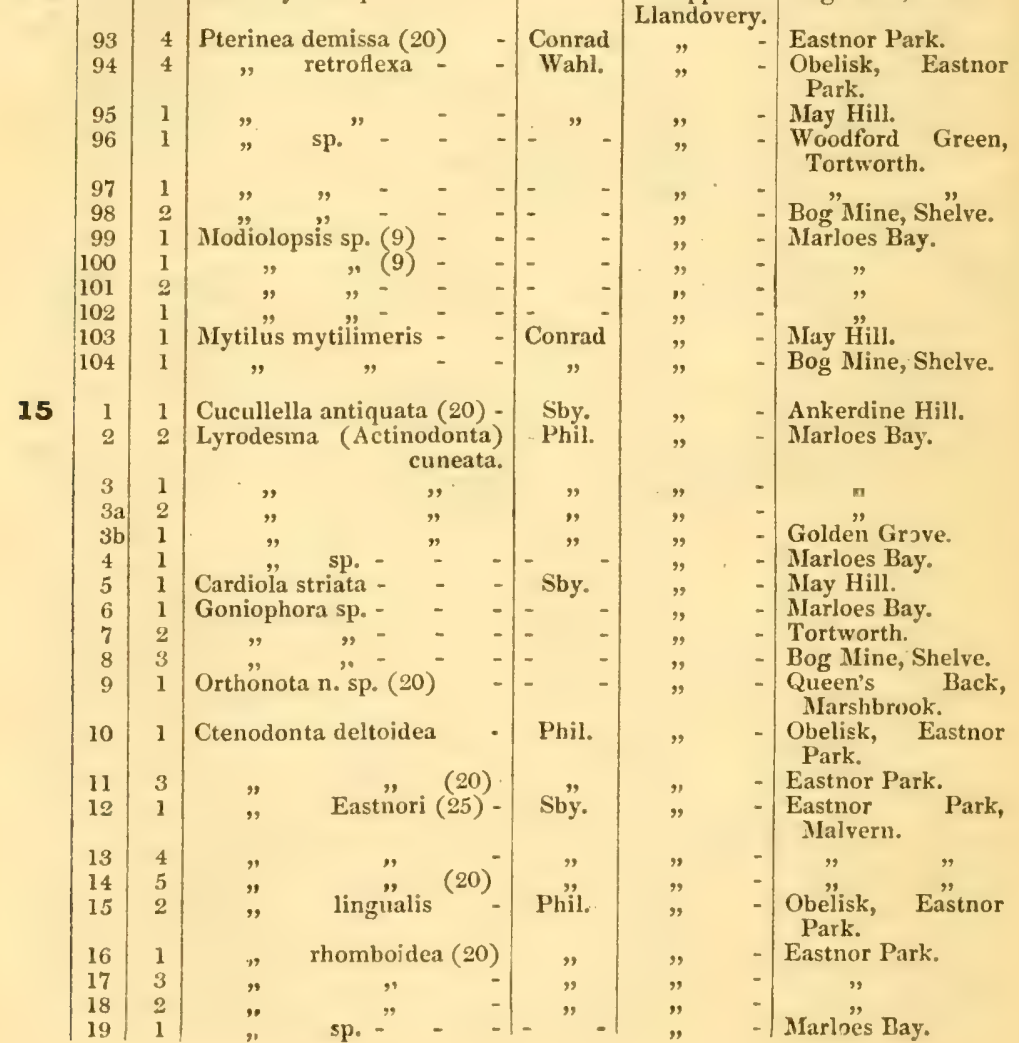




\begin{tabular}{|c|c|c|c|c|c|c|}
\hline مै & 造 & 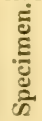 & Name. & $\begin{array}{c}\text { Author } \\
\text { of } \\
\text { Species. }\end{array}$ & Formation. & Locality. \\
\hline
\end{tabular}

LAMELLIBRANCHYATA-cont.

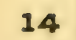

Flat

Case.
15

\section{Drawer}

14

\section{GASTEROPODA.}

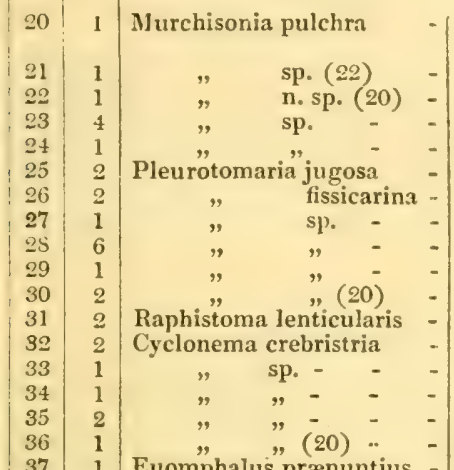

Euomphalus prænuntius - Phil.

sculptus (20)

"

n. $"$ sp. (20)

Trochonema trochleata - $\mathbf{M}^{\circ} \mathrm{Coy}$

Turbo tritorquatus

Acroculia haliotis (20)

Holopella cancellata -

(20) -

"

"

(20) -

48
49

49

50

51

53

39 obsoleta

.

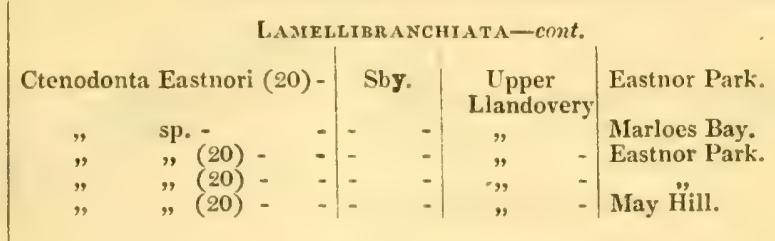

40

41
42

43

44
$\mathbf{M}^{6} \mathrm{Coy} \begin{gathered}\text { Upper } \\ \text { Llandovery. }\end{gathered} \mid$ May Hill.

Eastnor Park.

Bog Mine, Shelve.

Norbury.

Gunwick Mill.

- Norbury.

Bog Mine, Shelve.

Pen-y-lan.

Norbury.

Nashscar,Presteign.

Damory Bridge.

Marshbrook.

- Gunwick Mill, Llandovery.

- May Hill.

- Presteign.

- Penlan Quarry.

- Penlan, Llandovery.

Bog Mine, Shelve.

Presteign.

- Norbury.

- Gunwick Mill, Malvern.

- Presteign.

- Howler's Heath.

- Charfield Green, Tortworth.

- Tortworth.

- Bog Mine, Shelve.

- Nashscar, Presteign.

Castell craig gwyddon.

- Nashscar,Presteign.

- May Hill.

Hope Quarry, Minsterly.

Damory Bridge, Tortworth.

- May Hill. 


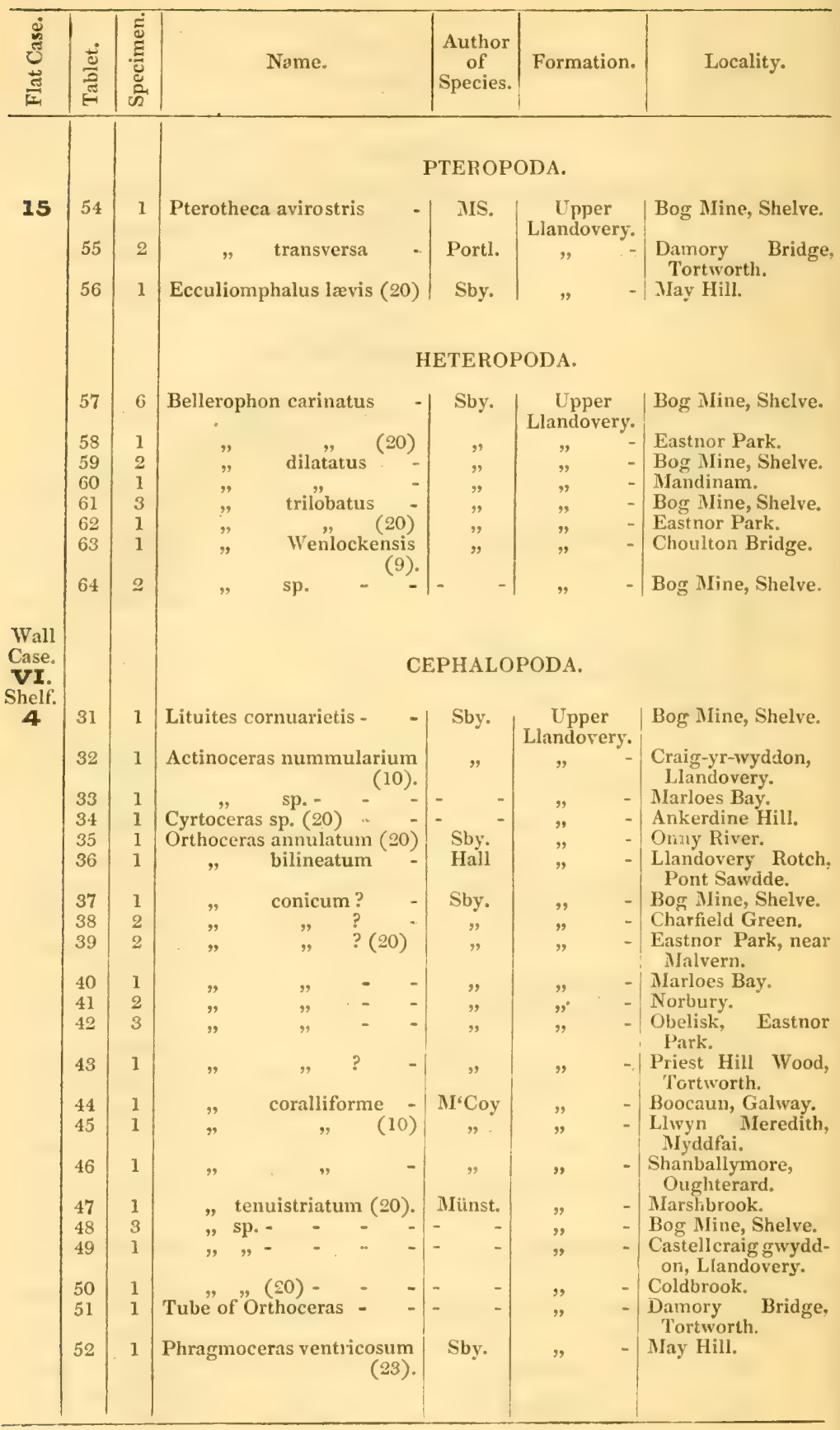




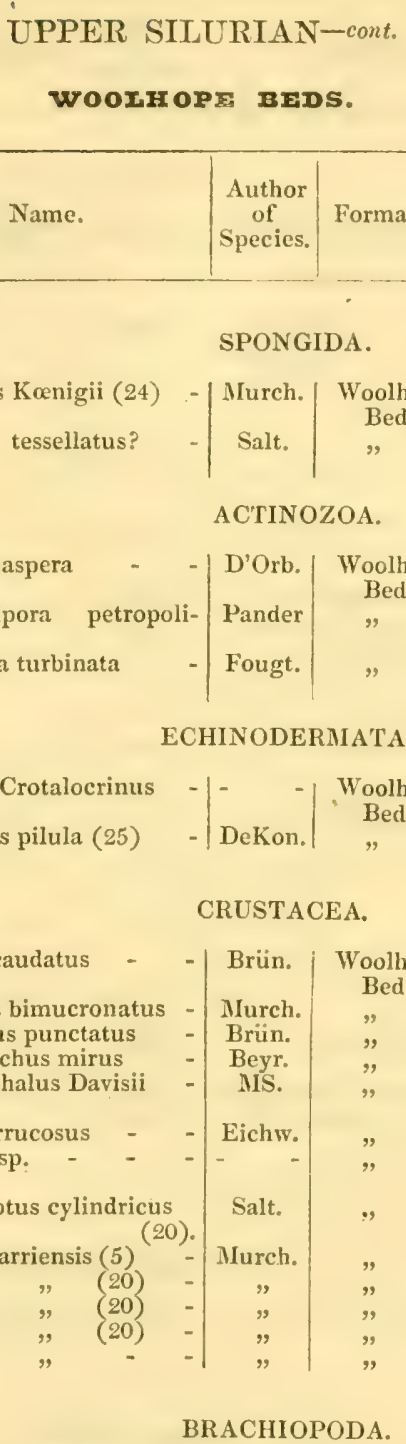

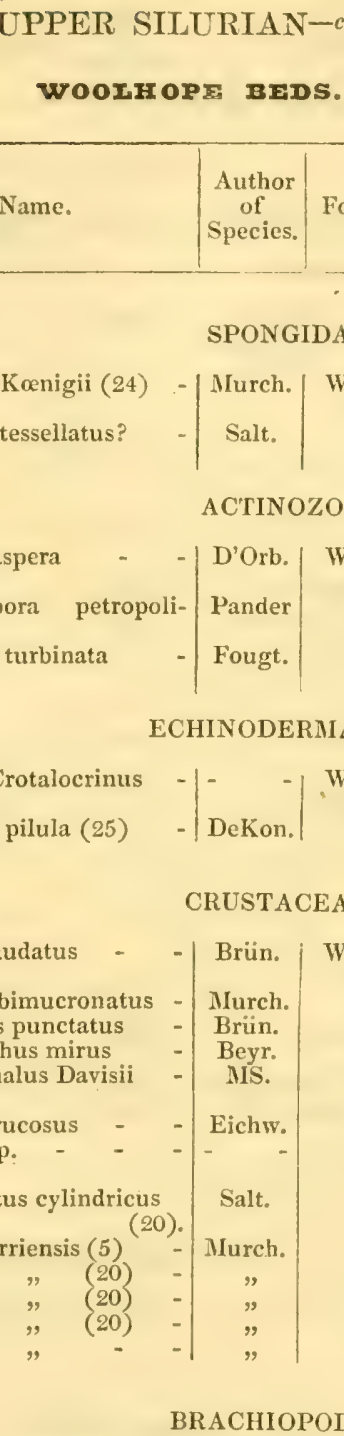

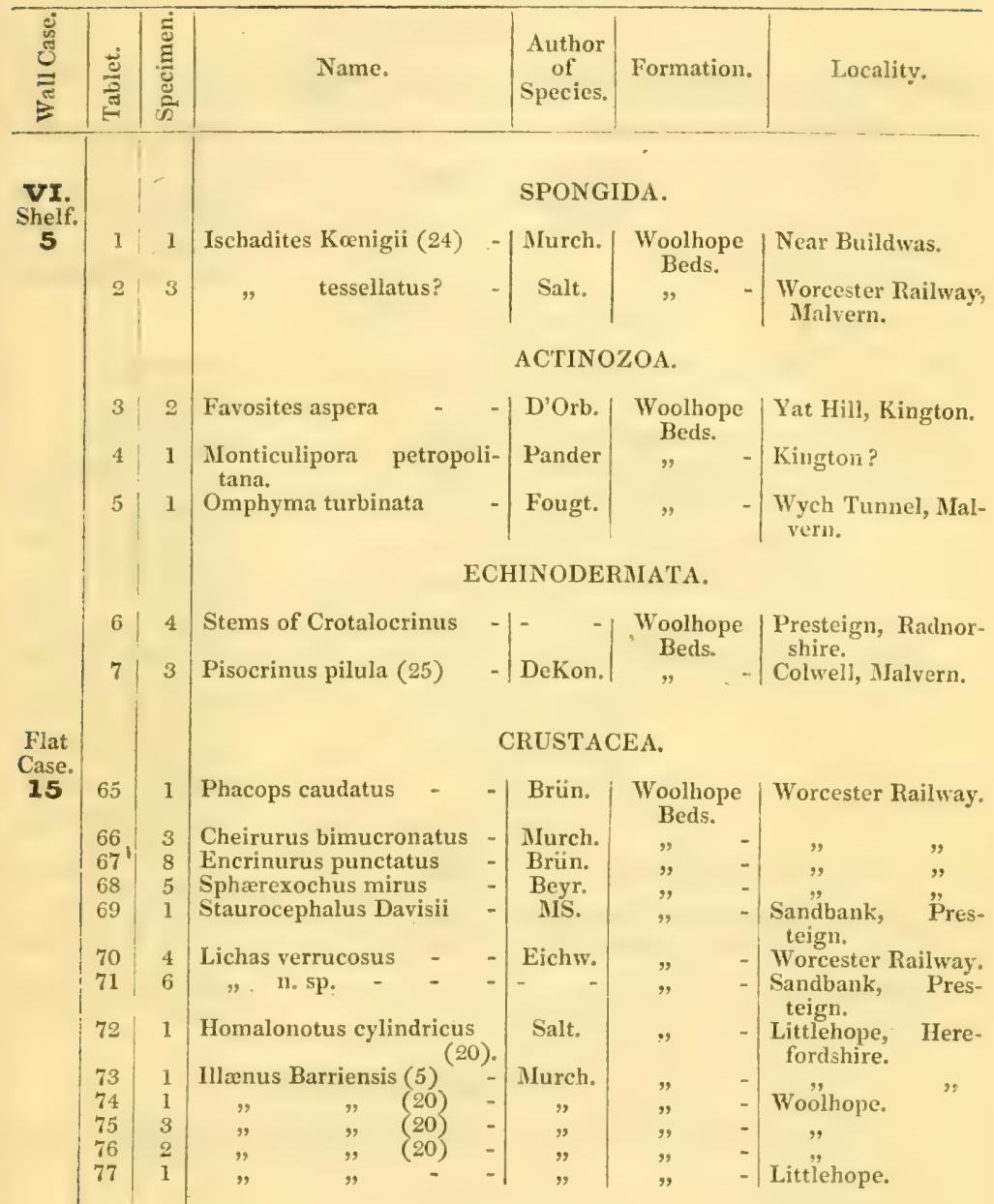

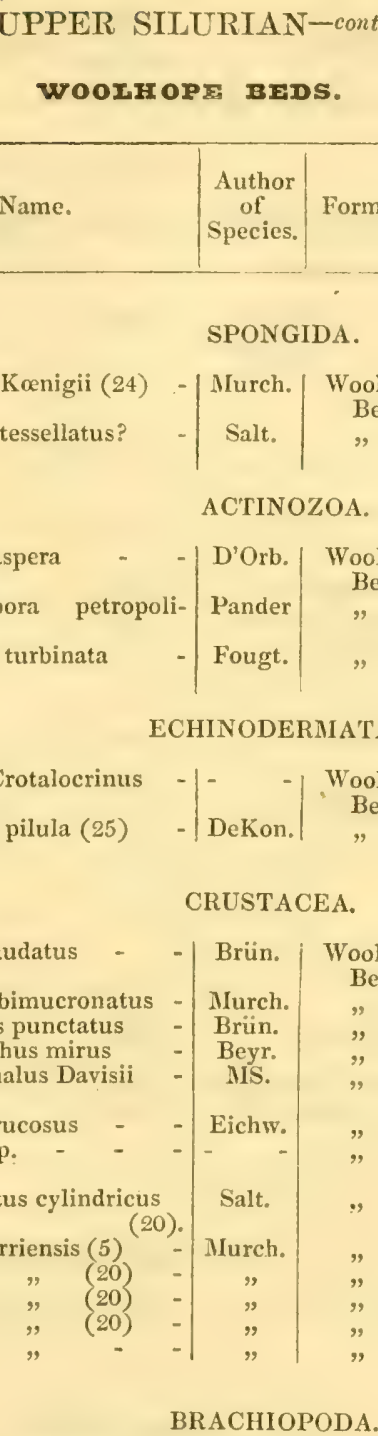

Meristella didyma (44)

- Dalm 


\begin{tabular}{|c|c|c|c|c|c|c|}
\hline 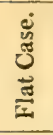 & है & 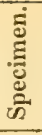 & Name. & $\begin{array}{c}\text { Author } \\
\text { of } \\
\text { Species. }\end{array}$ & Formation & Locality. \\
\hline \multirow{7}{*}{15} & & & \multicolumn{4}{|c|}{ BRACHOPODA-cont. } \\
\hline & 85 & 2 & Rhynchone!la borealis & Schloth. & $\begin{array}{l}\text { Woolhope } \\
\text { Beds. }\end{array}$ & $\begin{array}{l}\text { Littlehope, Wool- } \\
\text { hope. }\end{array}$ \\
\hline & 86 & 2 & $" \quad(44)$ & " & & $\begin{array}{l}\text { Ballard's Quarry, } \\
\text { Woolhope. }\end{array}$ \\
\hline & 87 & 1 & deflexa (44) & Sby. & " & - " " " \\
\hline & 88 & 2 & Stricklandi & $"$ & $"$ & - Nashscar,Presteign. \\
\hline & 89 & 4 & Wilsoni & $"$ & " & 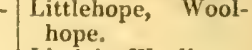 \\
\hline & 90 & 2 & " & , & , & - Lindels, Woolhope. \\
\hline \multirow[t]{17}{*}{16} & 1 & 1 & Stricklandinia lirata - & " & ", & - Woolhope. \\
\hline & $\begin{array}{l}2 \\
3\end{array}$ & 1 & $" \quad "(20)-$ & " & $"$ & \\
\hline & $\begin{array}{l}3 \\
4\end{array}$ & 7 & Triplesia Macoyana - & Dav. & ", & - Near Malvern. \\
\hline & 5 & 4 & Leptæna transversalis & Wahl. & ", & S. of Woolhope. \\
\hline & 6 & 1 & Orthis calligramma - & Dalm. & $"$ & $\begin{array}{l}\text { - Hope Quarry, Min- } \\
\text { sterly. }\end{array}$ \\
\hline & 7 & 1 & $"$ & " & $"$ & $\begin{array}{l}\text { W. of Wych Tun- } \\
\text { nel, Malvern. }\end{array}$ \\
\hline & 8 & 4 & $"$ reversa - & Salt. & , & - $\begin{array}{l}\text { Sandbanks, Pres- } \\
\text { teign. }\end{array}$ \\
\hline & 9 & 2 & " rustica - & Sby. & " & - Dursley Cross. \\
\hline & 10 & 2 & Strophomena euglypha & His. & $"$ & $\begin{array}{l}\text { Worcester Railway, } \\
\text { Malvern. }\end{array}$ \\
\hline & 11 & 1 & pecten & Linn. & ", & - Sandbanks, Pres- \\
\hline & 12 & 2 & rhomboidalis & Wilck. & " & $\begin{array}{l}\text { Worcester Railway, } \\
\text { Malvern. }\end{array}$ \\
\hline & 13 & 1 & , (20) & " & " & - Woolhope. \\
\hline & 14 & 8 & $"$ & ” & $"$ & $\begin{array}{l}\text { - Chickley Common, } \\
\text { Woolhope. }\end{array}$ \\
\hline & 15 & 3 & Chonetes striatella - & Dalm. & $"$ & $\begin{array}{l}\text { E. of Merchlin, } \\
\text { Conway. }\end{array}$ \\
\hline & 16 & 1 & Orbiculoidea Forbesii (44) & Dav. & " & - Malvern. \\
\hline & 17 & 1 & Lingula Symondsii (45) & Salt. & $"$ & 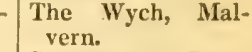 \\
\hline & 18 & 1 & " & $"$ & $"$ & - $\begin{array}{l}\text { Sandbanks, Pres- } \\
\text { teign. }\end{array}$ \\
\hline
\end{tabular}

\section{LAMELLIBRANCHIATA.} 191 Mytilus mytilimeris - $\quad-\mid$ Conrad $\left|\begin{array}{c}\text { Woolhope } \\ \text { Beds. }\end{array}\right| \begin{gathered}\text { Sandbanks, Pres- } \\ \text { teign. }\end{gathered}$

GASTEROPODA.

Euomphalus? sp. (5)

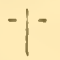

- Woolhope Beds.

Littlebope.

HETEROPODA. 


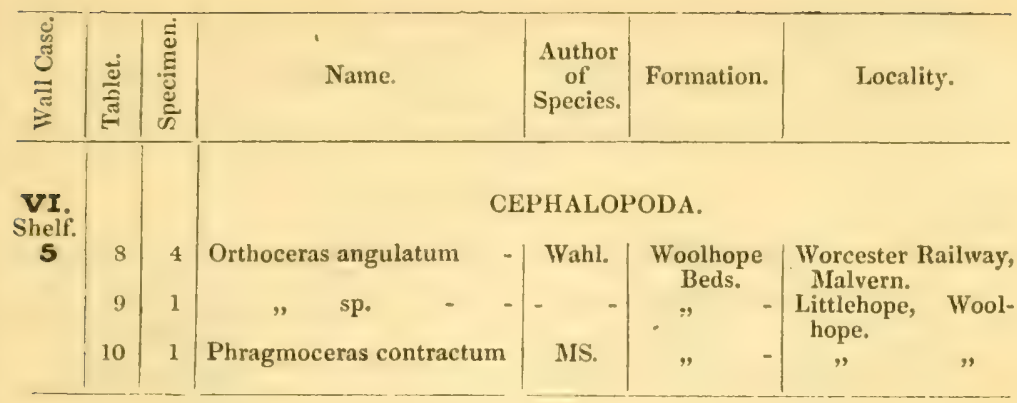

\section{UPPER SILURIAN-cont.}

WENIOCK SHATE.

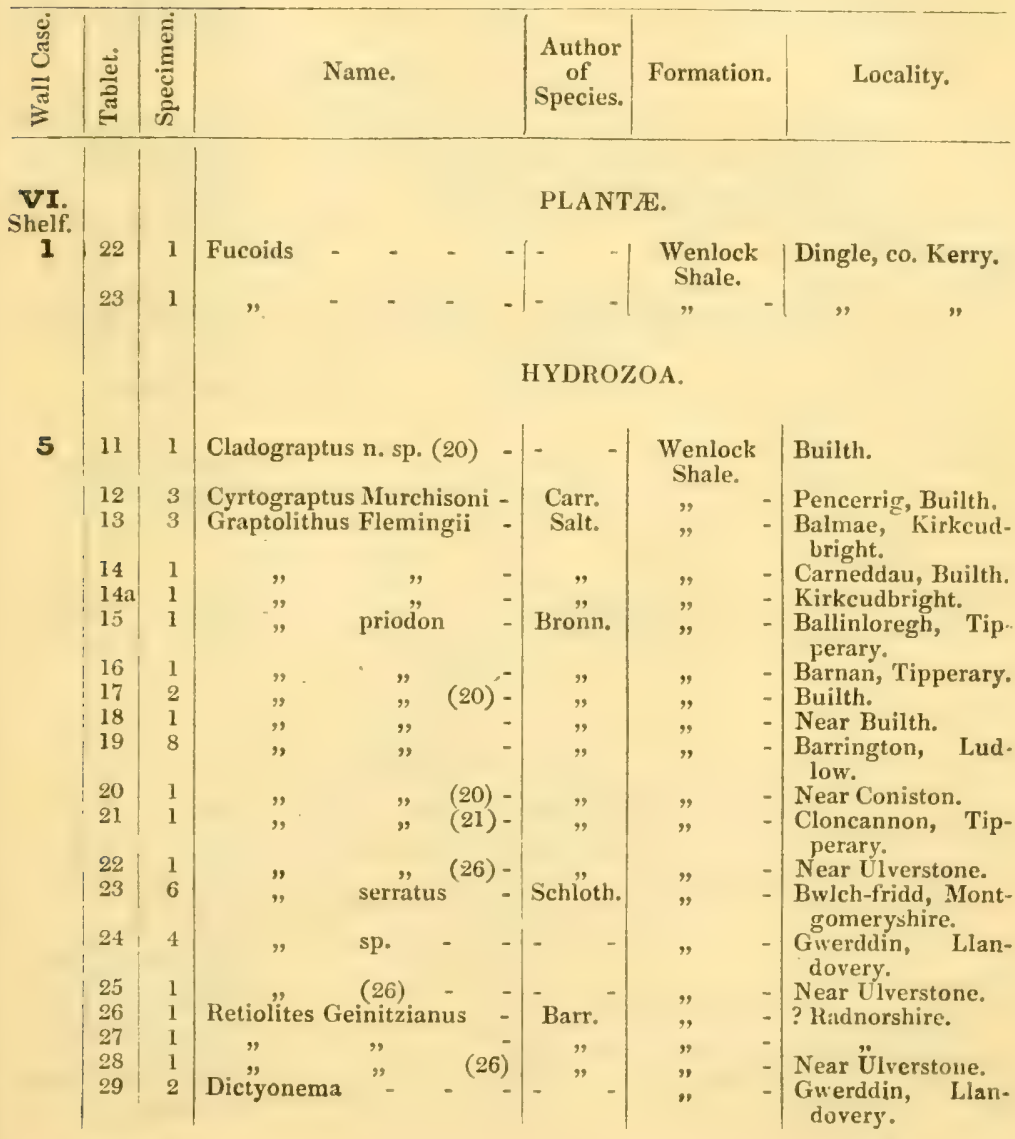




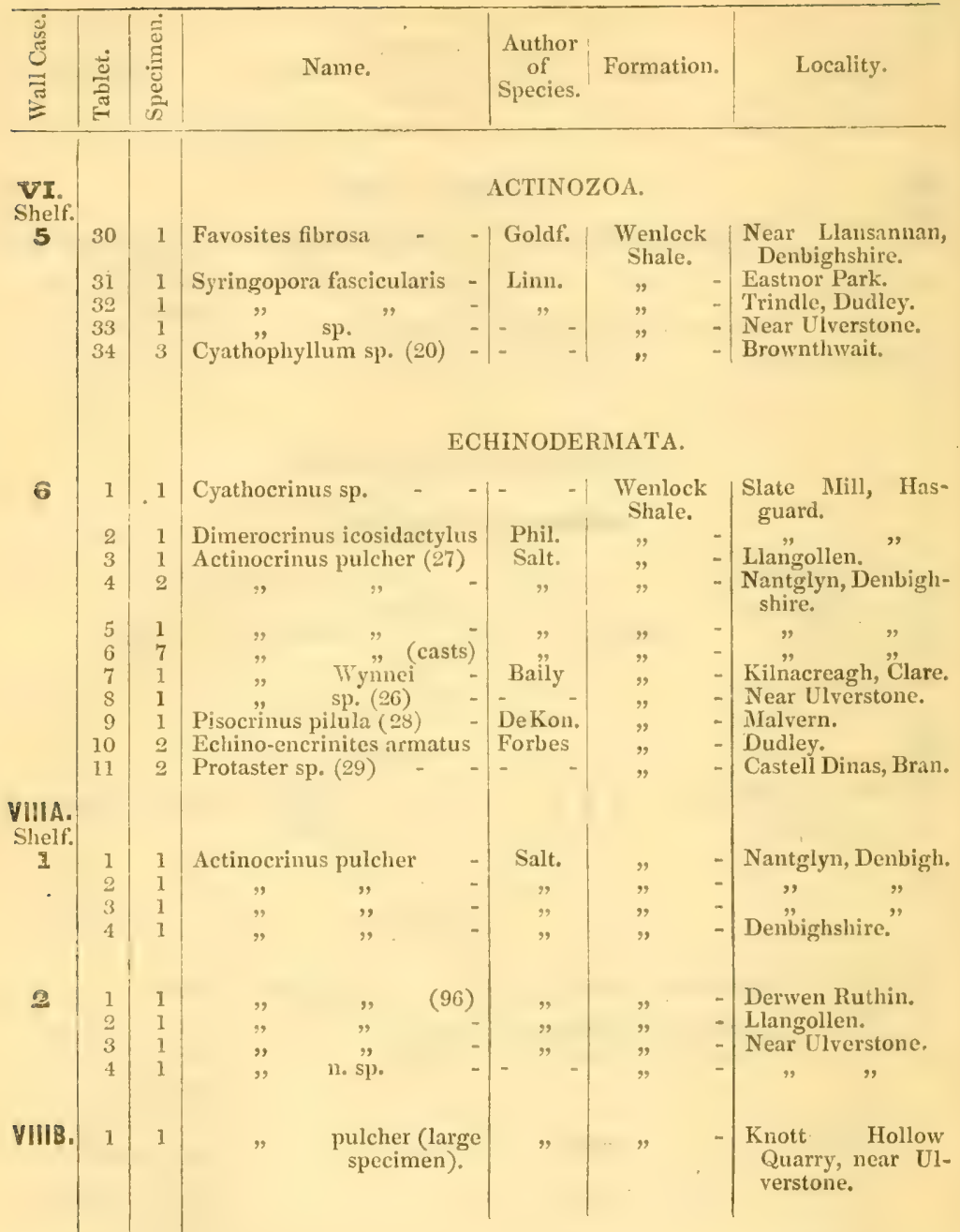

VT.

Shelf.

6
ANNELIDA.

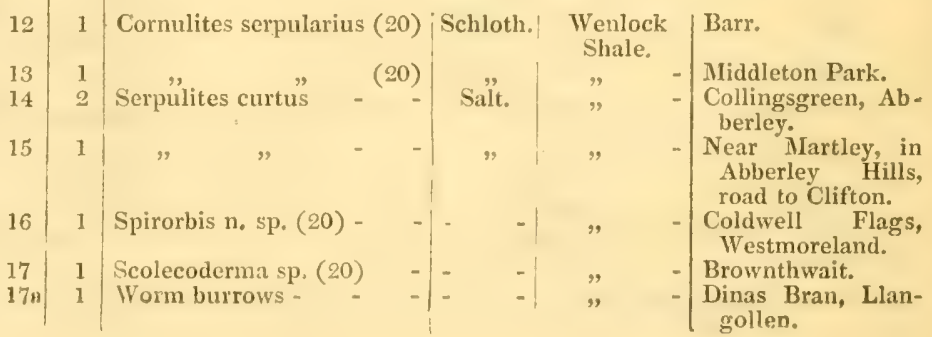




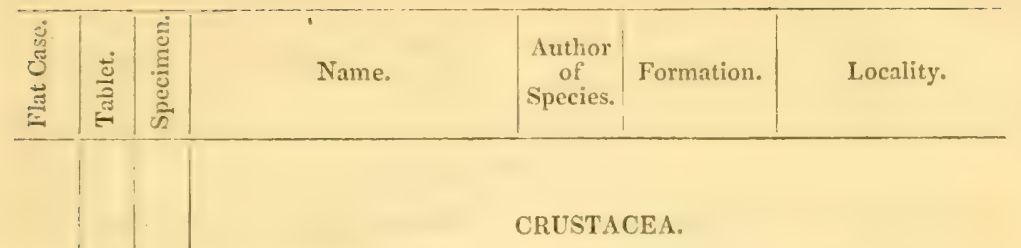

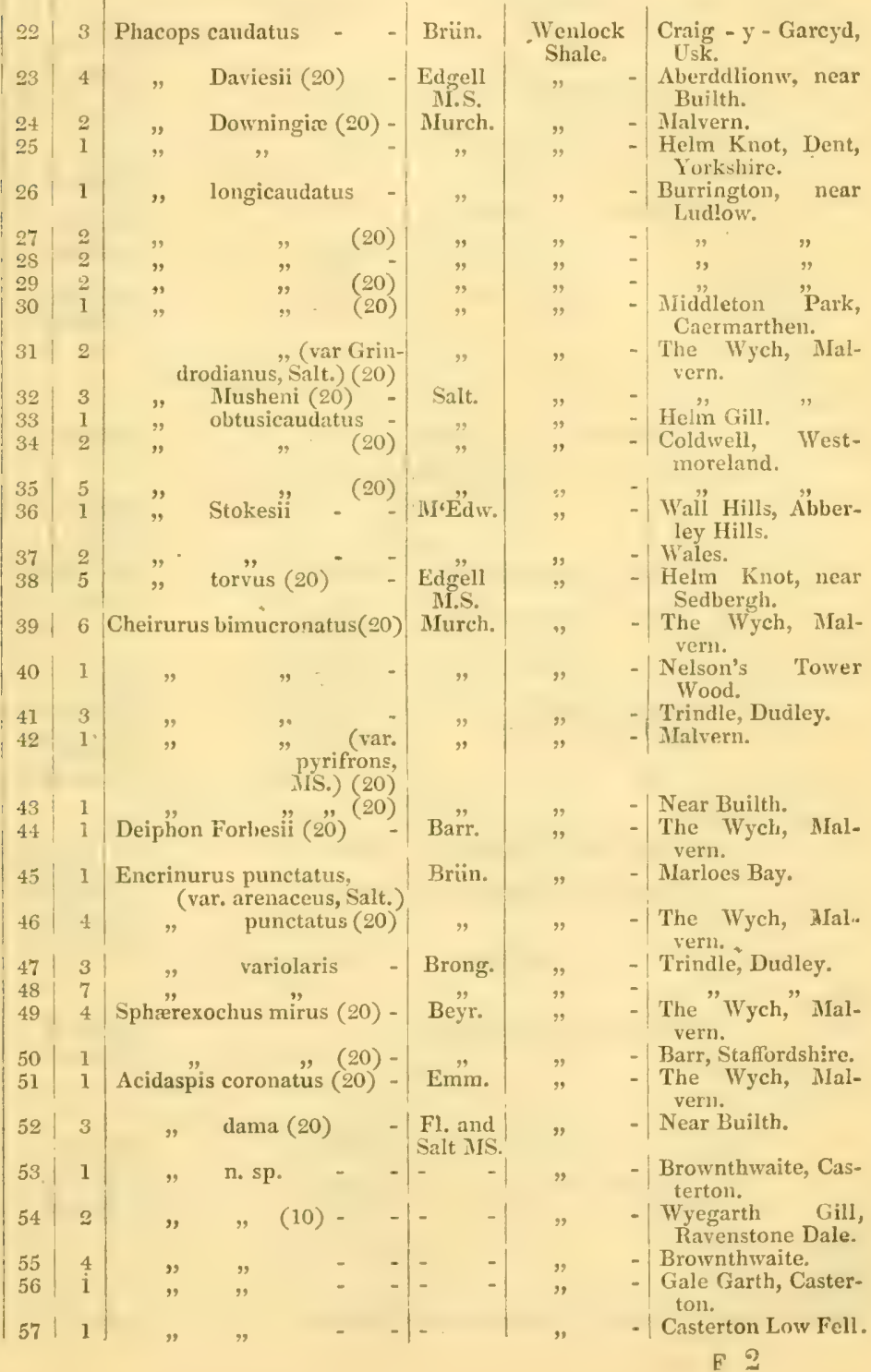




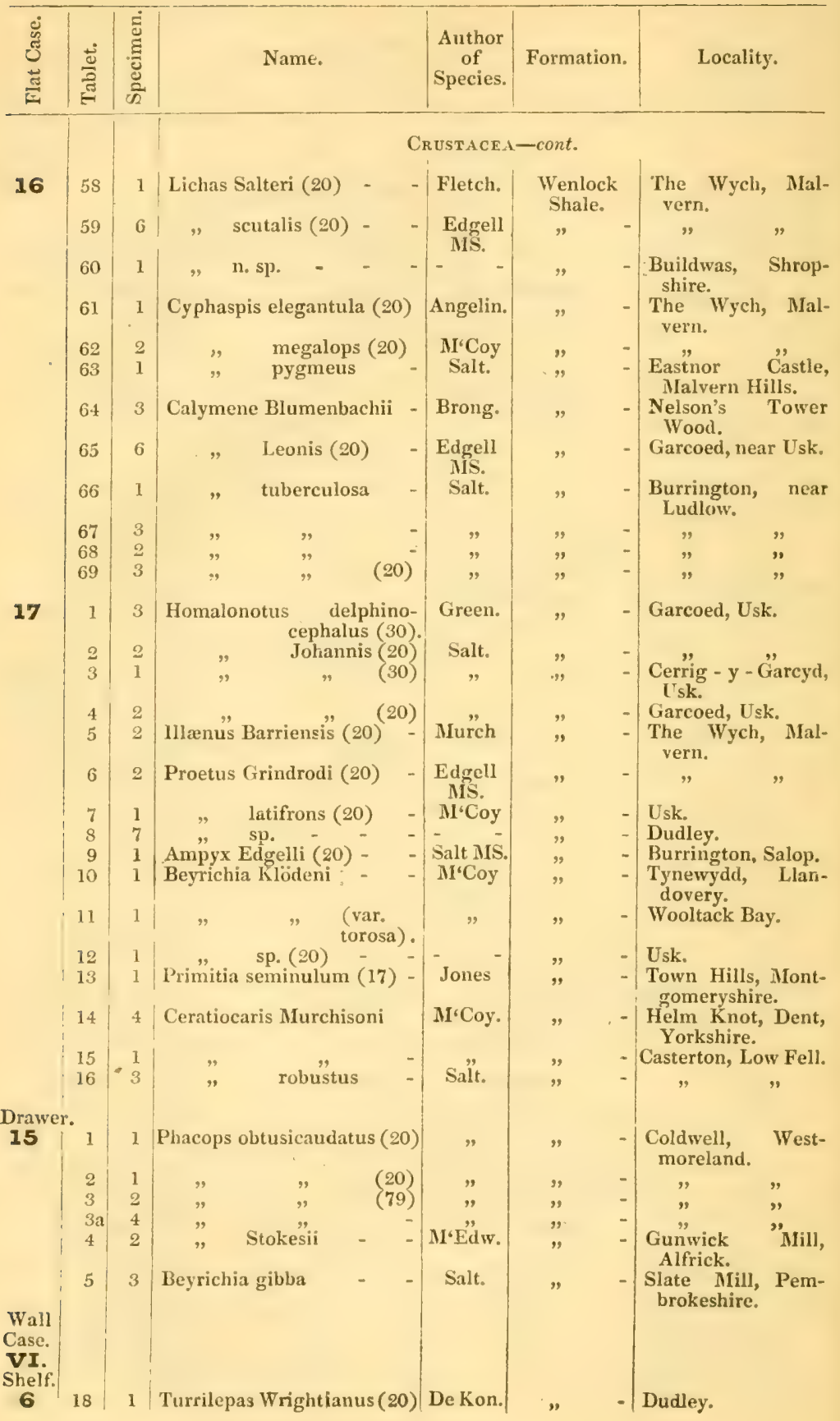




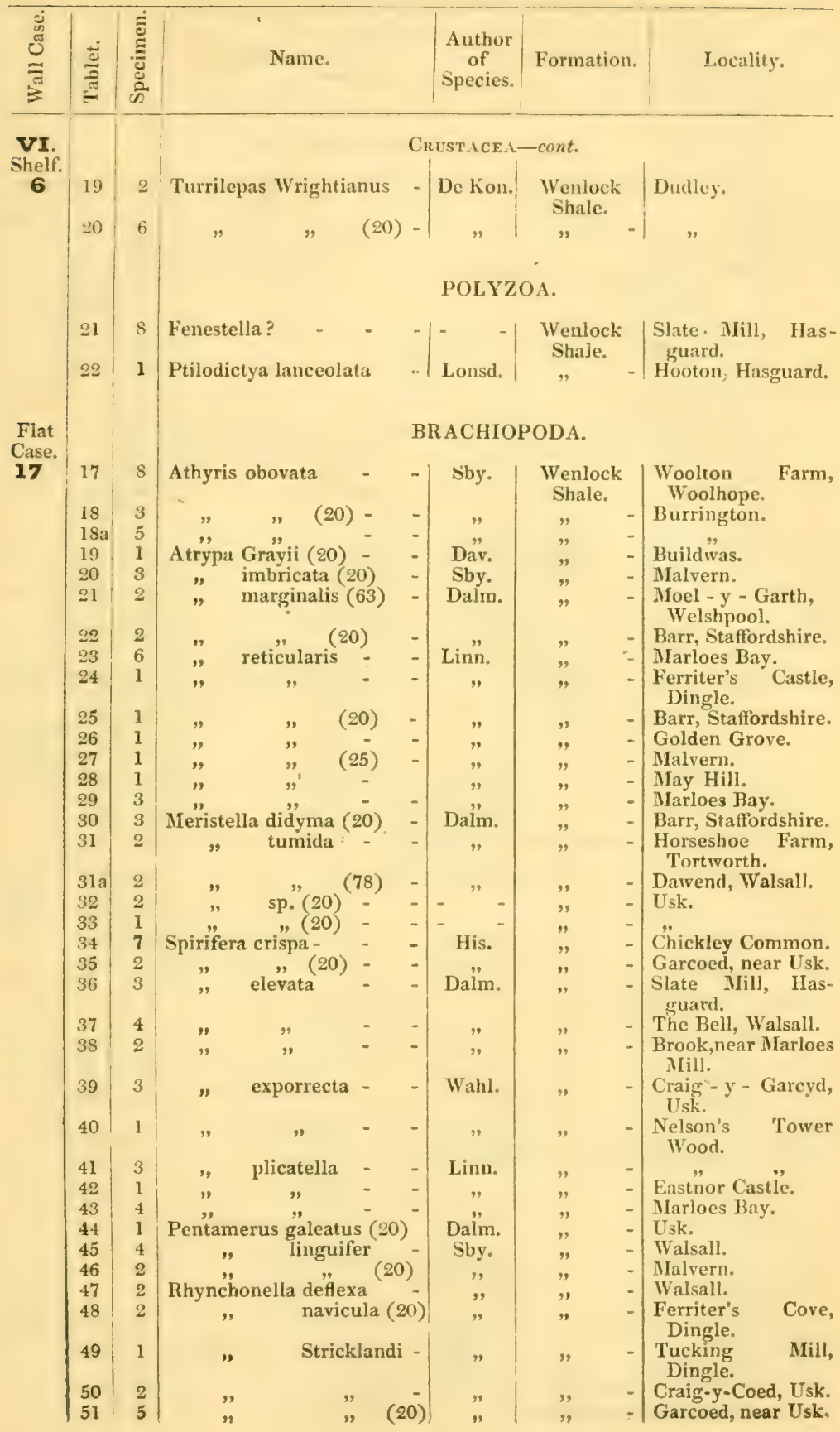




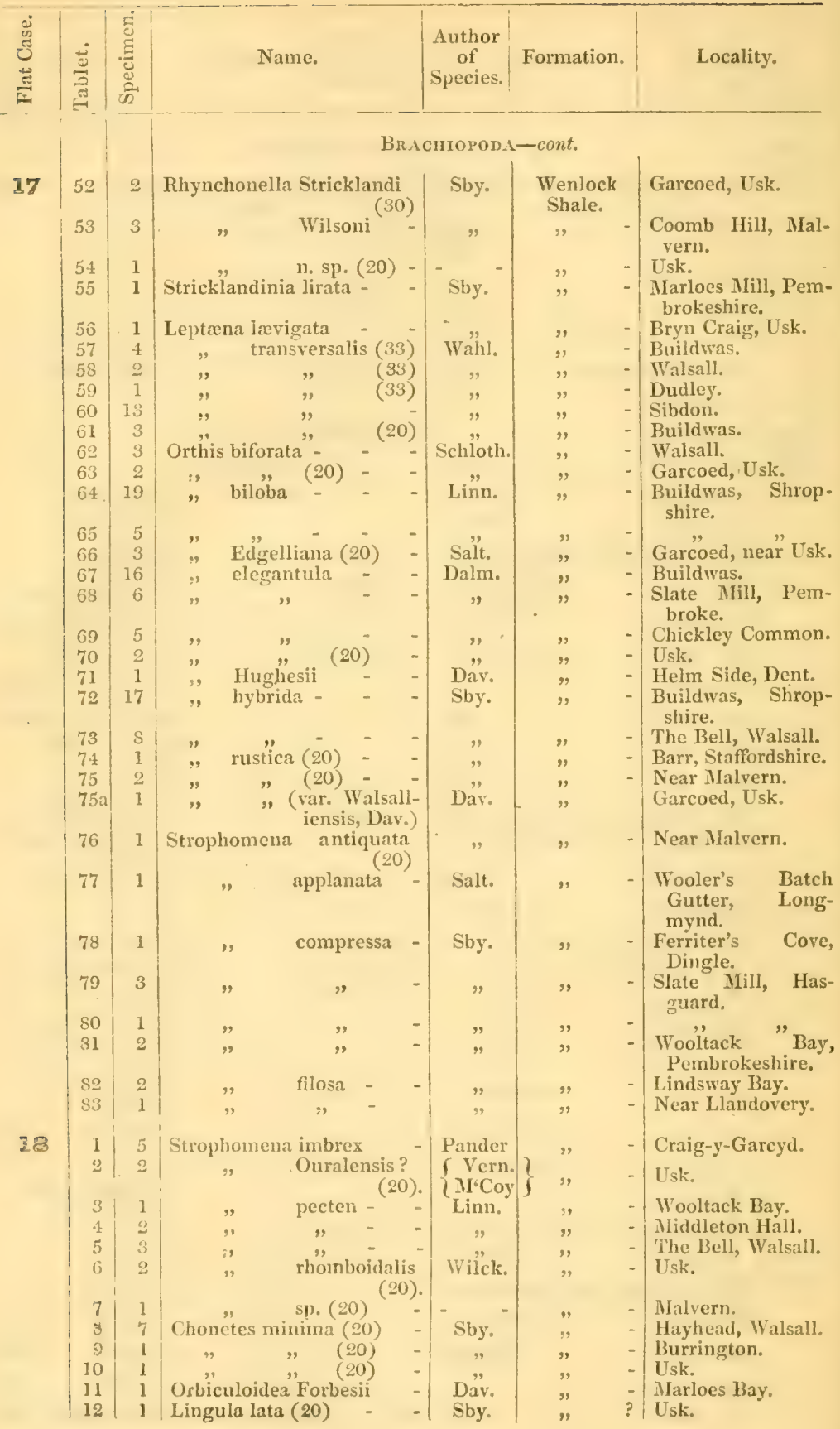




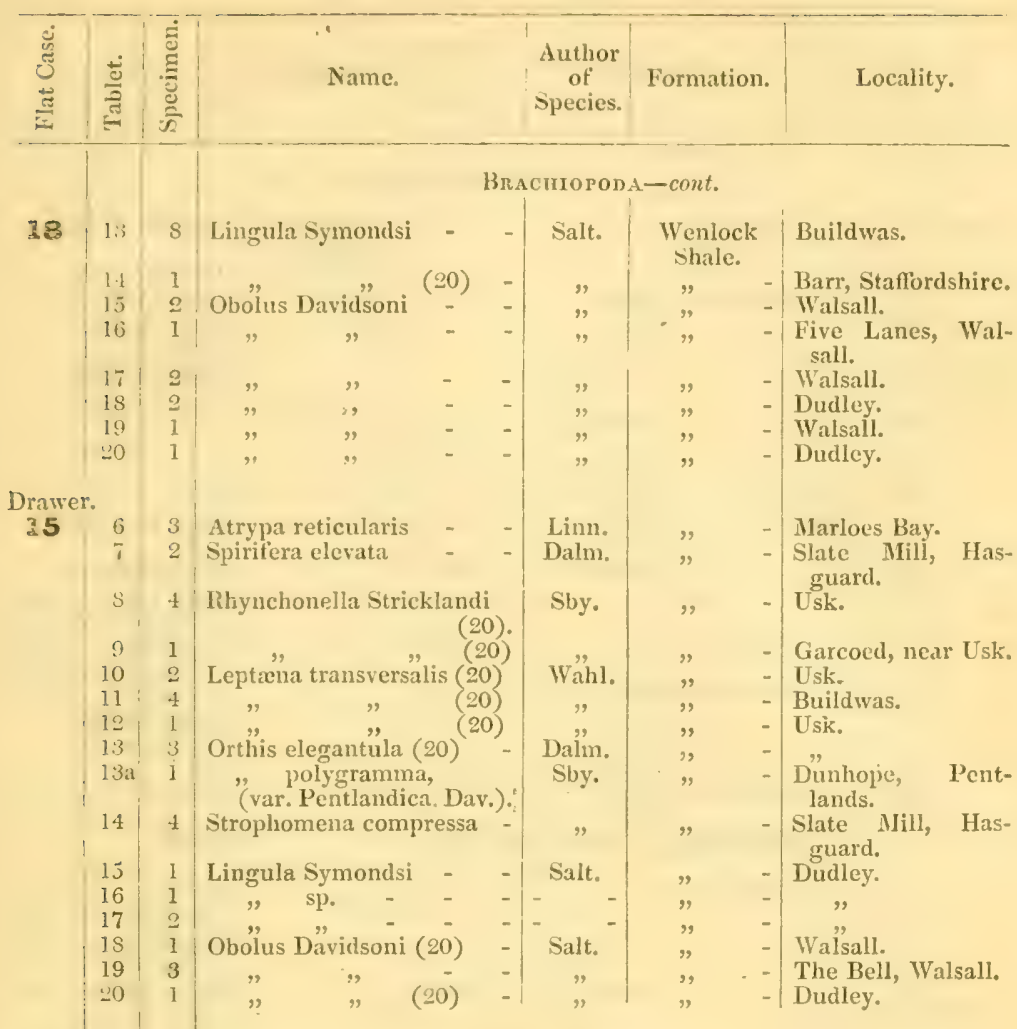

LANELLIBRANCHIATA. 


\begin{tabular}{|c|c|c|c|c|c|c|}
\hline 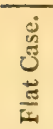 & 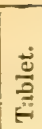 & 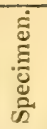 & Name. & $\begin{array}{c}\text { Author } \\
\text { of } \\
\text { Species. }\end{array}$ & Formation. & Locality. \\
\hline
\end{tabular}

\section{8

\begin{tabular}{|c|c|c|c|c|c|c|c|}
\hline \multirow[b]{2}{*}{38} & \multirow[b]{2}{*}{3} & \multicolumn{6}{|c|}{ LAMELLIBRANCHIATA - cont. } \\
\hline & & Anodontopsis sp. & & $-1=$ & $\begin{array}{l}\text { Wenlock } \\
\text { Shale. }\end{array}$ & & Coombe Hill. \\
\hline 39 & 2 & " $\quad$ (2 & & $-1=$ & $"$ & - & Garcoed, Usk. \\
\hline 40 & 1 & Modiolopsis gradata & & - Salt. & $"$ & - & Bryn Craig, Usk. \\
\hline 41 & 3 & $"$ & - & $"$ & $"$ & - & $\begin{array}{l}\text { Five Lanes, Wal- } \\
\text { sall. }\end{array}$ \\
\hline 42 & 1 & $"$ & (30) & " & $"$ & - & Garcoed, Usk. \\
\hline 43 & 2 & $"$. & (20) & & ” & - & Garcoed, Usk. \\
\hline 44 & 3 & Leonis & $(20)^{\prime}$ & $\begin{array}{l}\text { - Edgell } \\
\text { MS. }\end{array}$ & $"$ & - & $"$ \\
\hline 45 & 1 & n. sp. ( & 20) & $-\mid-$ & " & - & $"$ \\
\hline 4 & 2 & sp. (20) & & $-i$ & " & - & Usk. \\
\hline $\begin{array}{l}47 \\
48\end{array}$ & $\begin{array}{l}2 \\
3\end{array}$ & $\begin{array}{l}\text { Mytilus chemungen } \\
\text { mytilimeris }\end{array}$ & sis & - Conrad & , & - & $\begin{array}{l}\text { Bryn Craig, Usk. } \\
\text { Usk. }\end{array}$ \\
\hline 49 & 1 & "? unguiculat & us & Salt. & $"$ & - & Bryn Craig, Usk. \\
\hline 50 & 1 & " sp. - & & $-{ }_{-}-$ & ", & - & $\begin{array}{l}\text { Lindsway Bay, } \\
\text { Pembrokeshire. }\end{array}$ \\
\hline 51 & 3 & Arca subxqualis & - & $-\mathbf{M}^{6} \mathrm{Coy}$ & " & - & $\begin{array}{l}\text { Nelson's Tower } \\
\text { Wood, Llandeilo. }\end{array}$ \\
\hline 52 & 1 & Cucullella antiqua & (20) & Sby. & " & - & $\begin{array}{l}\text { Middleton Park, } \\
\text { Caermarthen. }\end{array}$ \\
\hline 53 & 1 & Cawdori & - & ", & " & - & Lindsway Bay. \\
\hline 54 & 5 & , sp. (20) & - & -- & " & - & Usk: \\
\hline 55 & 5 & Palæarca sp. (20) & - & -- & " & - & Garcoed, Usk. \\
\hline $\begin{array}{l}56 \\
57\end{array}$ & 1 & Pseudoaxinus ${ }^{(20)}$ con & spicuus & - & $"$ & $=$ & Dudley." \\
\hline 37 & 1 & Psendoaxinus con & $\begin{array}{l}\text { spicuus } \\
(20)\end{array}$ & 1s. M.S. & " & -1 & Dudley. \\
\hline 58 & 1 & Cardiola fibrosa & - & Sby. & " & - & $\begin{array}{l}\text { Burrington, near } \\
\text { Ludlow. }\end{array}$ \\
\hline 59 & 1 & $"$ & - & $"$ & $"$ & - & $\begin{array}{l}\text { Nantglyn, Den- } \\
\text { bighshire. }\end{array}$ \\
\hline 60 & 4 & interrupta & - & Brod. & " & - & $\begin{array}{l}\text { Ross Side, near } \\
\text { Ulverstone. }\end{array}$ \\
\hline 61 & 2 & $"$ & - & " & " & - & $\begin{array}{l}\text { Garcoed and Wen- } \\
\text { lock. }\end{array}$ \\
\hline 62 & 3 & " & - & " & $"$ & - & $\begin{array}{l}\text { Oakley, Farm, } \\
\text { Bishop's Castle. }\end{array}$ \\
\hline $\begin{array}{l}63 \\
64\end{array}$ & $\begin{array}{l}2 \\
1\end{array}$ & $\begin{array}{c}\text { n. sp. - } \\
\text {," }\end{array}$ & 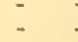 & $=$ & $"$ & - & $\begin{array}{l}\text { Builth. } \\
\text { Tyn-y-pwll, Llan- }\end{array}$ \\
\hline & & Lunulacardium alif, & & Sby. & 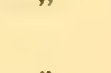 & & $\begin{array}{l}\text { syn-y-pwi, } \\
\text { sannan, Denbigh- }\end{array}$ \\
\hline 65 & 1 & Lunulacardium alit & orme & soy. & " & - & $\begin{array}{l}\text { Oakley Farm, } \\
\text { Bishop's Castle. }\end{array}$ \\
\hline 66 & 1 & sp. & - & - & " & - & $\begin{array}{l}\text { Burrington, near } \\
\text { Ludlow. }\end{array}$ \\
\hline 67 & $\begin{array}{l}1 \\
\mathbf{2}\end{array}$ & Goniophora cymbre & formis & Sby. & " & - & Craig-y-Garcyd. \\
\hline 69 & 1 & $\begin{array}{l}\text { Grammysia angulat: } \\
\text { Orthonota inornata }\end{array}$ & & $\begin{array}{l}\text { His. } \\
\text { Phil. }\end{array}$ & $"$ & 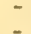 & Bryn Craig, Usk. \\
\hline 70 & 1 & $" \quad$ sp. $(30)$ & - & $-{ }_{-}$ & ," & - & Usk Tunnel. \\
\hline 71 & 9 & Ctenodonta Ainglica & $1(20)$ & - D'Orb. & $"$ & - & Garcoed, near Usk. \\
\hline 72 & 1 & " & $(20)$ & & ", & -1 & Barr, Staffordshire. \\
\hline 73 & 1 & sulcata & & His. & " & - & $\begin{array}{l}\text { Slate Mill, Has- } \\
\text { guard. }\end{array}$ \\
\hline 71 & 1 & $50,(20)$ & (20) & $"$ & $"$ & - & Garcoed, Usk. \\
\hline 76 & 1 & $\begin{array}{l}\text { sp. (20) } \\
",(20)\end{array}$ & - & - & $"$ & - & $\begin{array}{l}\text { Usk. } \\
\text { Barr. }\end{array}$ \\
\hline 21 & 1 & Pterinea tenuistriat & & M'Coy & $"$ & - & Casterton Low Fell. \\
\hline 22 & 1 & " & - & $"$ & " & - & $\begin{array}{l}\text { Straight Bridge, } \\
\text { Sedbergh. }\end{array}$ \\
\hline
\end{tabular}




\begin{tabular}{|c|c|c|c|c|c|c|}
\hline 离 & है & 岂 & Name. & $\begin{array}{l}\text { Author } \\
\text { of } \\
\text { Species. }\end{array}$ & Formation. & Locality. \\
\hline
\end{tabular}

\section{5}

I'lat

Case

18

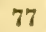

\section{LAMELLIBRANCHIATA-cont.}

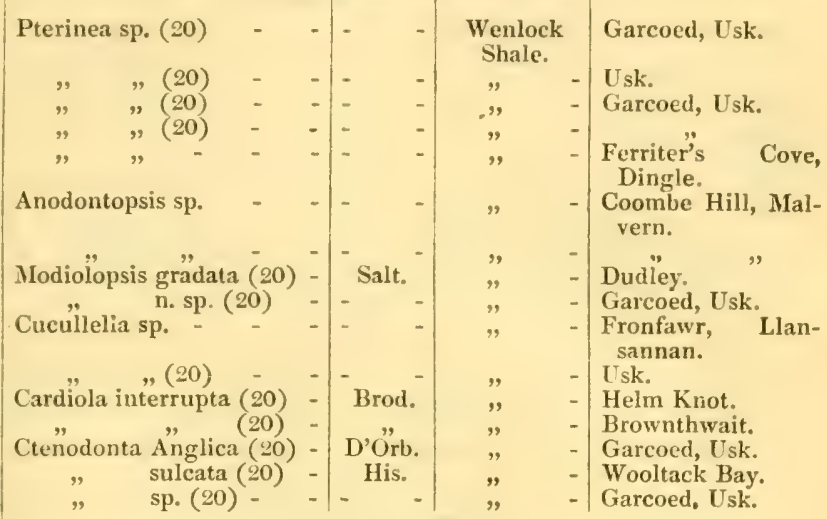

\section{GASTEROPODA.}

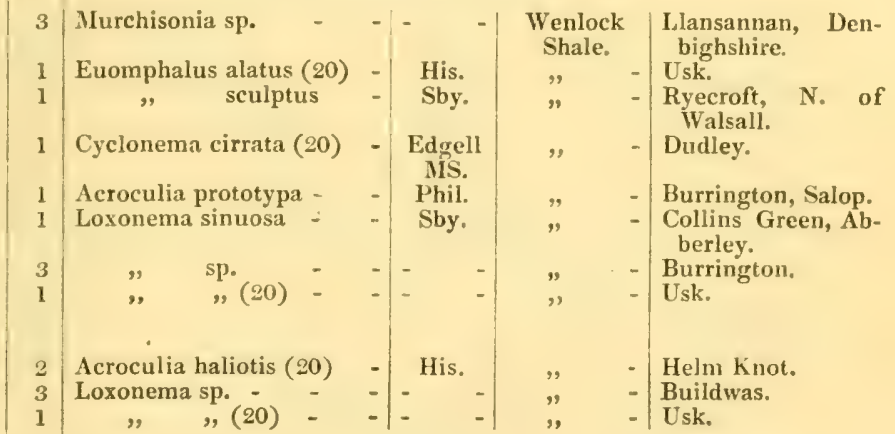

\section{PTEROPODA.}

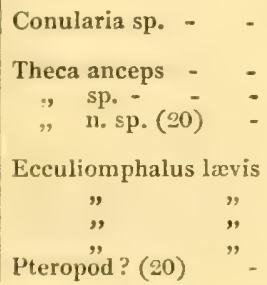

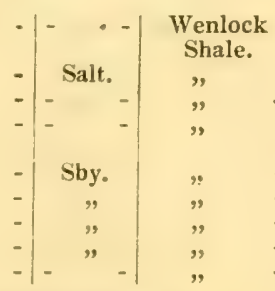

Usk.

Eastnor Castle.

- River Wye, Builth.

- Aberddlionw, near Builth.

Paniel Hall Mill.

- Eastnor Castle.

Burrington.

- Usk."

\section{HETEROPODA.}

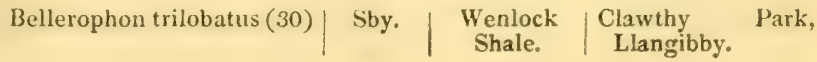




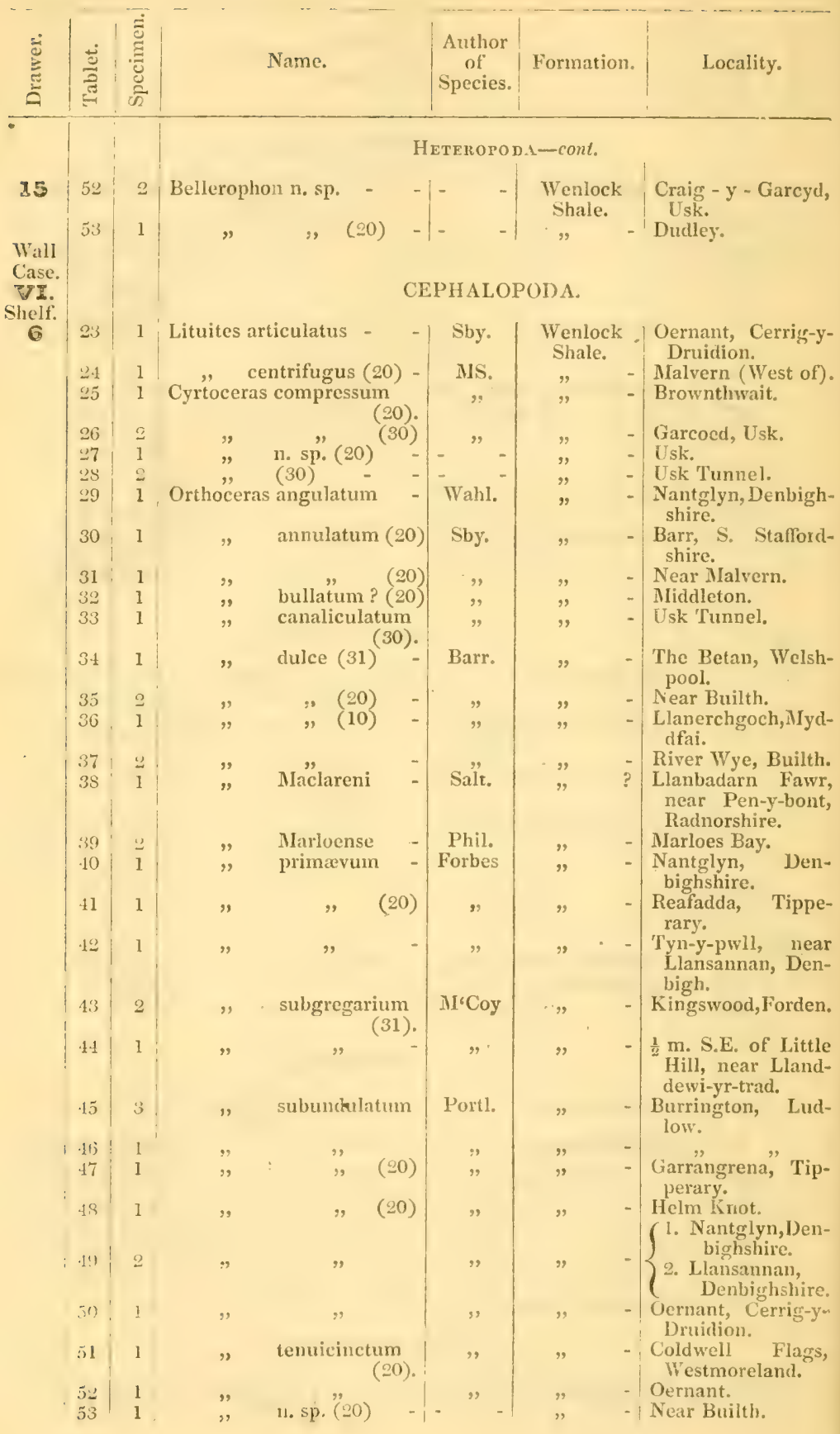




\begin{tabular}{|c|c|c|c|c|c|c|c|c|}
\hline$\underbrace{3}_{=}$ & $\frac{\vec{b}}{\frac{\omega}{5}}$ & 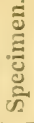 & & Name. & & $\begin{array}{c}\text { Author } \\
\text { of } \\
\text { Species. }\end{array}$ & Formation. & Locality. \\
\hline $\begin{array}{c}\text { VT. } \\
\text { Shelf. } \\
6\end{array}$ & $\begin{array}{l}58 \\
59 \\
60 \\
61 \\
62 \\
63\end{array}$ & $\begin{array}{l}3 \\
2 \\
3 \\
1 \\
3 \\
1\end{array}$ & $\begin{array}{c}\text { Orthoceras } \\
, " \\
, " \\
, " \\
, \\
\text { Aptychus? } \\
\text { Phragmoce } \\
\text {," }\end{array}$ & $\begin{array}{l}\text { sp. - } \\
",(20) \\
"(20) \\
"(20) \\
"(20) \\
"(20) \\
\text { of Ortho } \\
\text { eras naut } \\
\text { pyrif } \\
\text { vent }\end{array}$ & $\begin{array}{ll} & \\
& - \\
- & - \\
- & - \\
- & - \\
- & - \\
- & - \\
\text { oceras - } & \\
\text { ileum? } \\
\text { orme - } \\
\text { ricosum } \\
\text { (20). }\end{array}$ & \begin{tabular}{ll}
- & - \\
- & - \\
- & - \\
- & - \\
- & - \\
- & - \\
- & - \\
- & - \\
\multicolumn{2}{c}{ Sby. } \\
\multicolumn{2}{c}{, }
\end{tabular} & 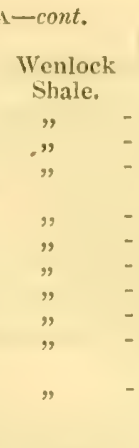 & $\begin{array}{l}\text { Buildwas. } \\
\text { Builth. } \\
\text { Carneddau, Builth. } \\
\text { Coldwell Flags, } \\
\text { Westmoreland. } \\
\qquad, \\
\text { ", ", } \\
\text { Builth. } \\
\text { Craig-y-Garcyd,Usk. } \\
\text { Five Lanes, Wal- } \\
\text { sall. } \\
\text { Malvern. }\end{array}$ \\
\hline
\end{tabular}

UPPER SILURIAN-cont.

WENIOCE JIMISTOND.

\begin{tabular}{|c|c|c|c|c|c|c|}
\hline 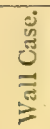 & $\frac{\frac{3}{3}}{\frac{3}{3}}$ & 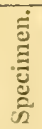 & Name. & $\begin{array}{l}\text { Author } \\
\text { of } \\
\text { Species. }\end{array}$ & Formation. & Locality. \\
\hline
\end{tabular}

VII.

Shelf.
VARIOUS LARGE SPECIMIENS.

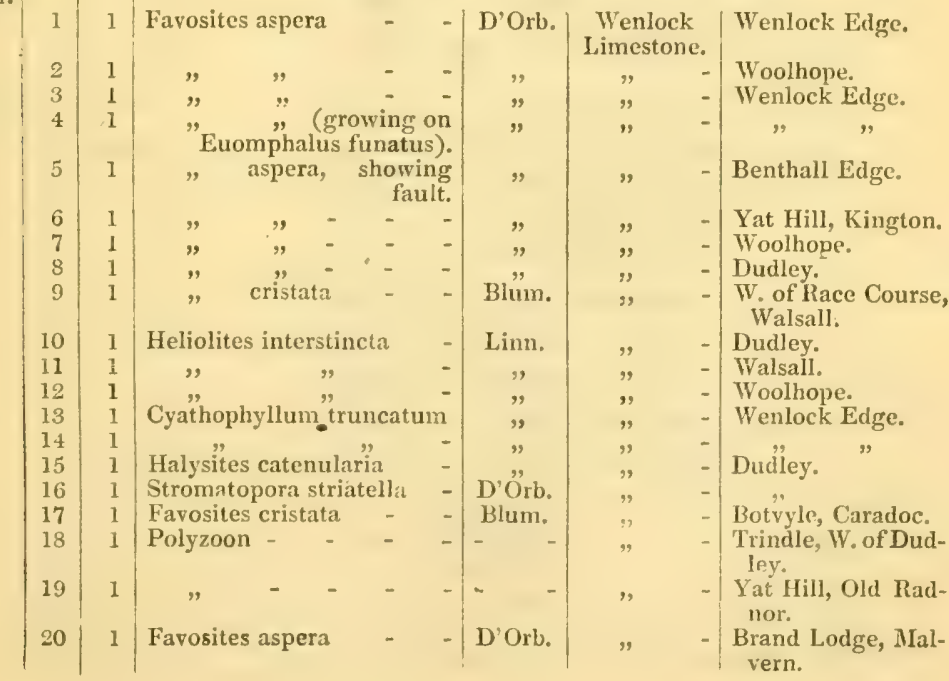




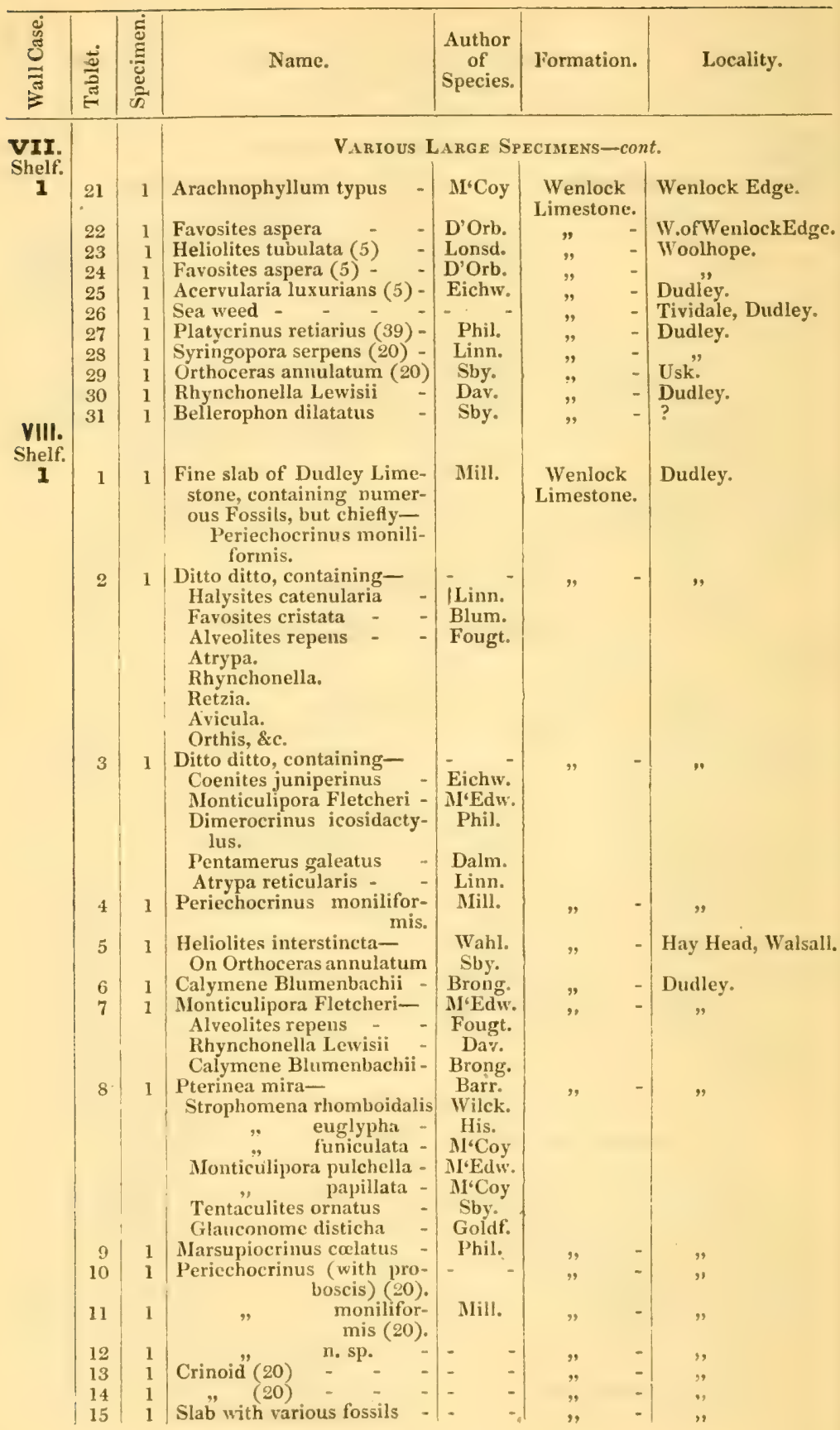




\begin{tabular}{|c|c|c|c|c|c|c|}
\hline 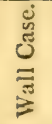 & 总 & 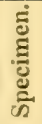 & Name. & $\begin{array}{c}\text { Author } \\
\text { of } \\
\text { Species. }\end{array}$ & Formation. & Locality. \\
\hline
\end{tabular}

VIII.

Shelf.

2

1

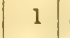

\section{aining- \\ Taxocrinus.}

Marsupiocrinus.

Glyptocrinus.

Acervularia.

Monticulipora, \&c.

21 Slab containing-

Pseudocrinites quadrifasciatus.

Heliolites Grayii?

M. Bowerbankii -

M. papillata

Syringopora bifurcata

Fenestella subantiqua

Various Large Specimens-cont.

Casts of Cystideans (95) -

Periechocrinus moniliformis.

3

VII.

SPONGIDA.
Monticulipora pulchella -

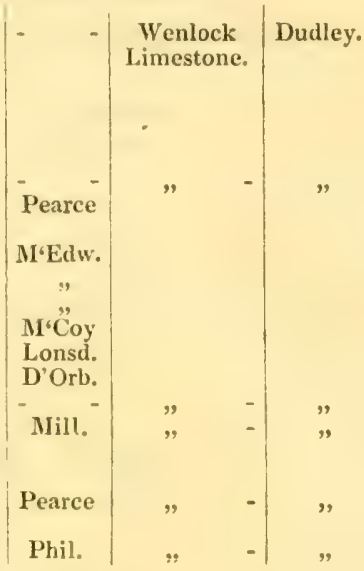

Stromatopora striatella (32) D'Orb. Wenlock Brand Lodge, Mal-

Limestone. vern.

"

"

DOrb.

Dudley.

- Near Dudley.

- Longhope, May Hill.

- Wenlock Edge.

Ischadites Konigï (20) - Murch.

Usk.

Dudley.

- Malvern.

HYDROZOA.

Graptolithus priodon -

- Bronn

\begin{tabular}{c|c} 
Wenlock & Dudley. \\
Limestone. &
\end{tabular}

\section{ACTINOZOA.}

$11 \quad 1$ Heliolites Grayi (20)

12 1 1 interstincta

13

14

15

16

17

18

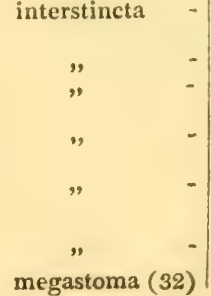

megastoma (32)

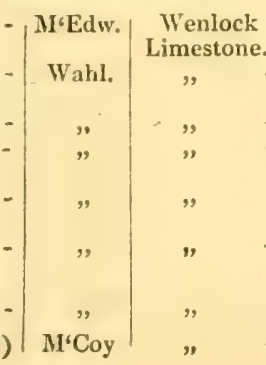

Dudley.

Brand Lodge, Malvern.

- Dudley.

f1. Red House, nr.

- Walsall.

2. Malvern Hills.

- Rock Farm, May

Hill.

f1. Walsall.

2. Dudley.

- I Malvern Hills. 


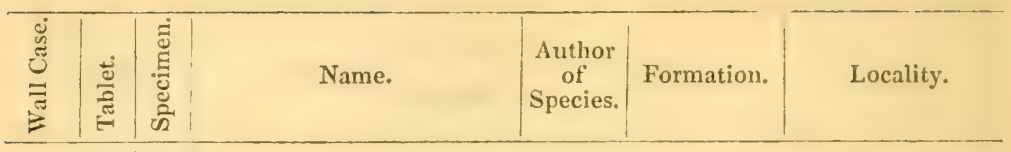

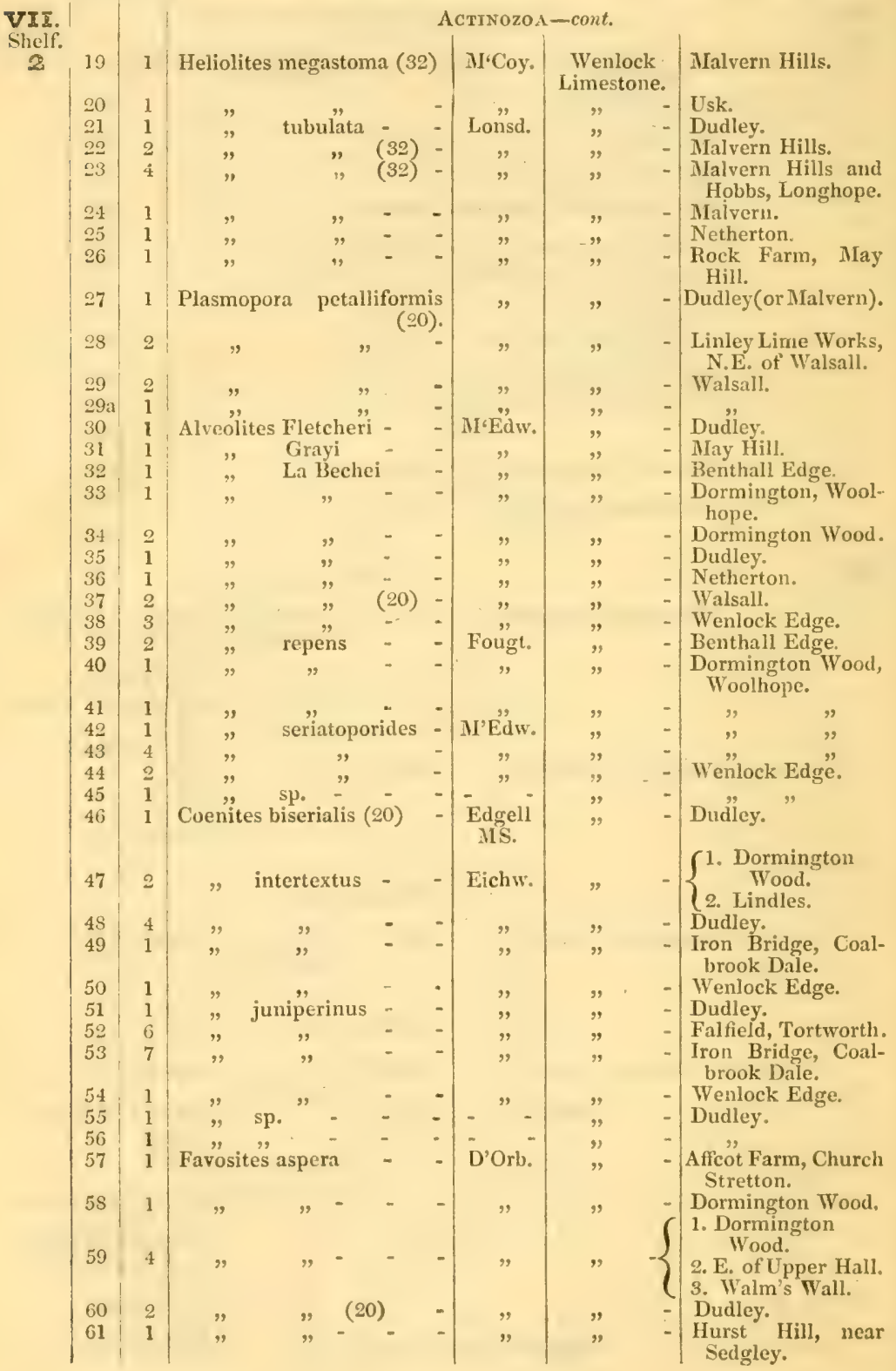




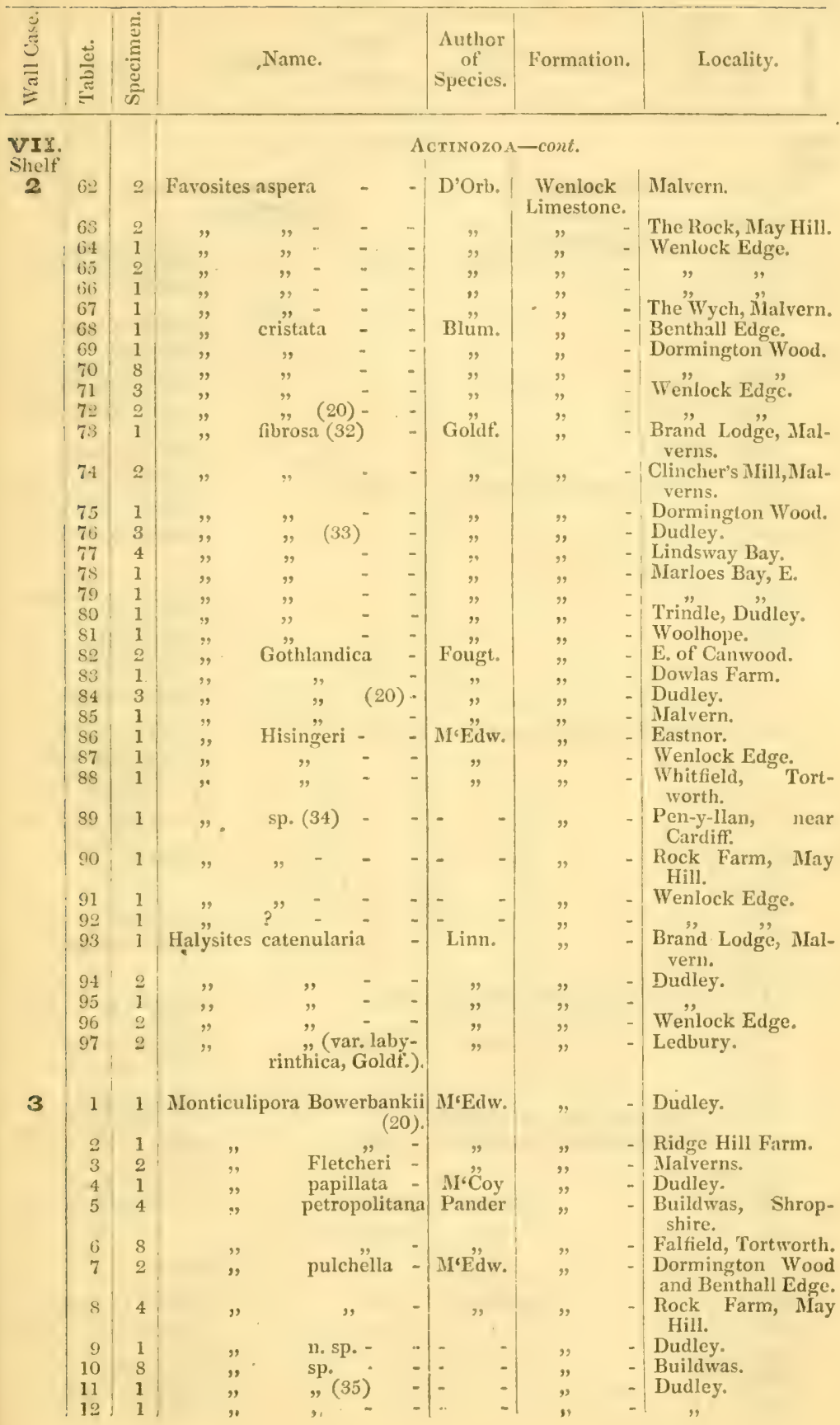




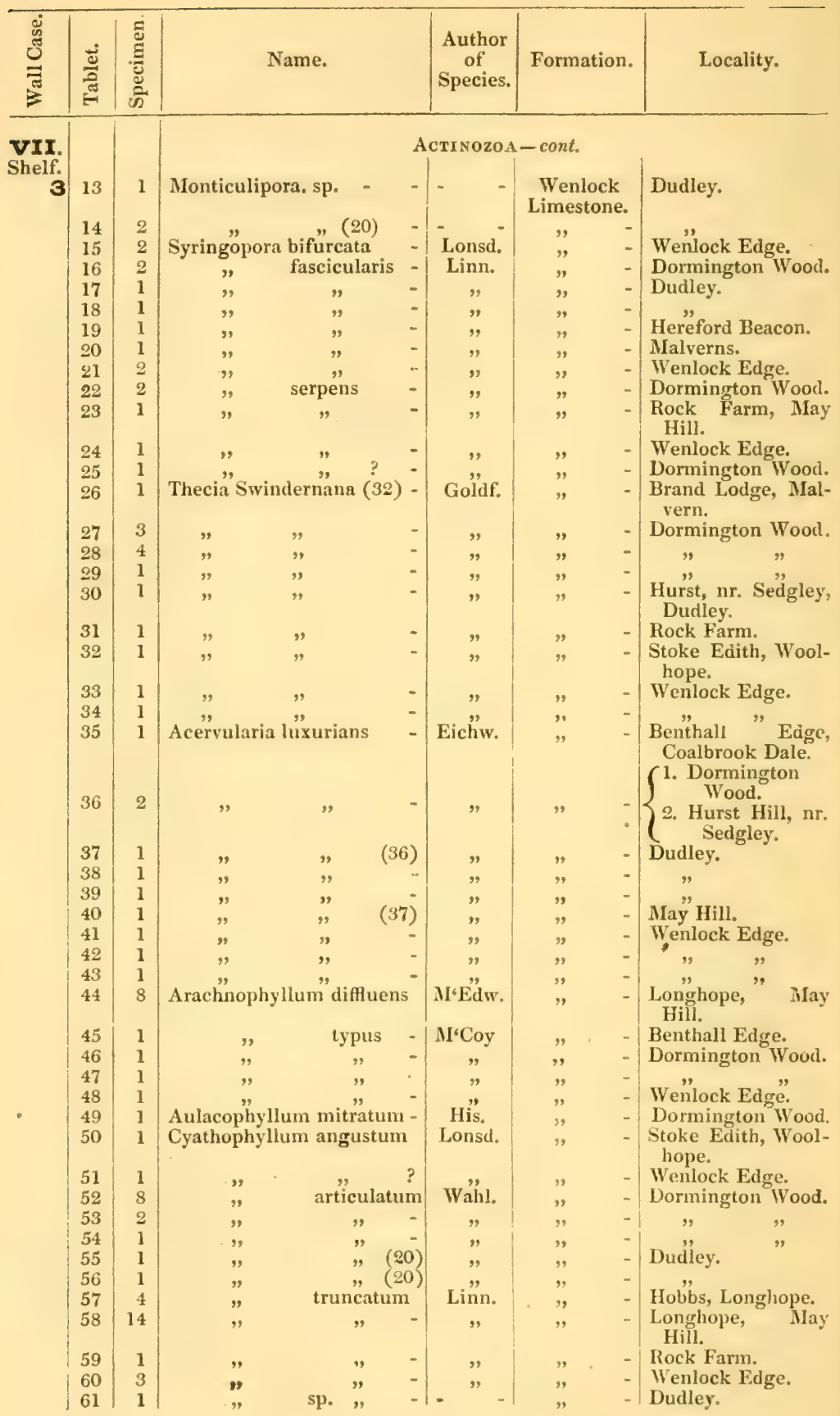




\begin{tabular}{|c|c|c|c|c|c|c|}
\hline 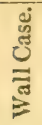 & ए। & 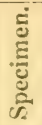 & Name. & $\begin{array}{l}\text { Author } \\
\text { of } \\
\text { Species. }\end{array}$ & Formation, & Locality. \\
\hline
\end{tabular}

VII. Shelf.
3

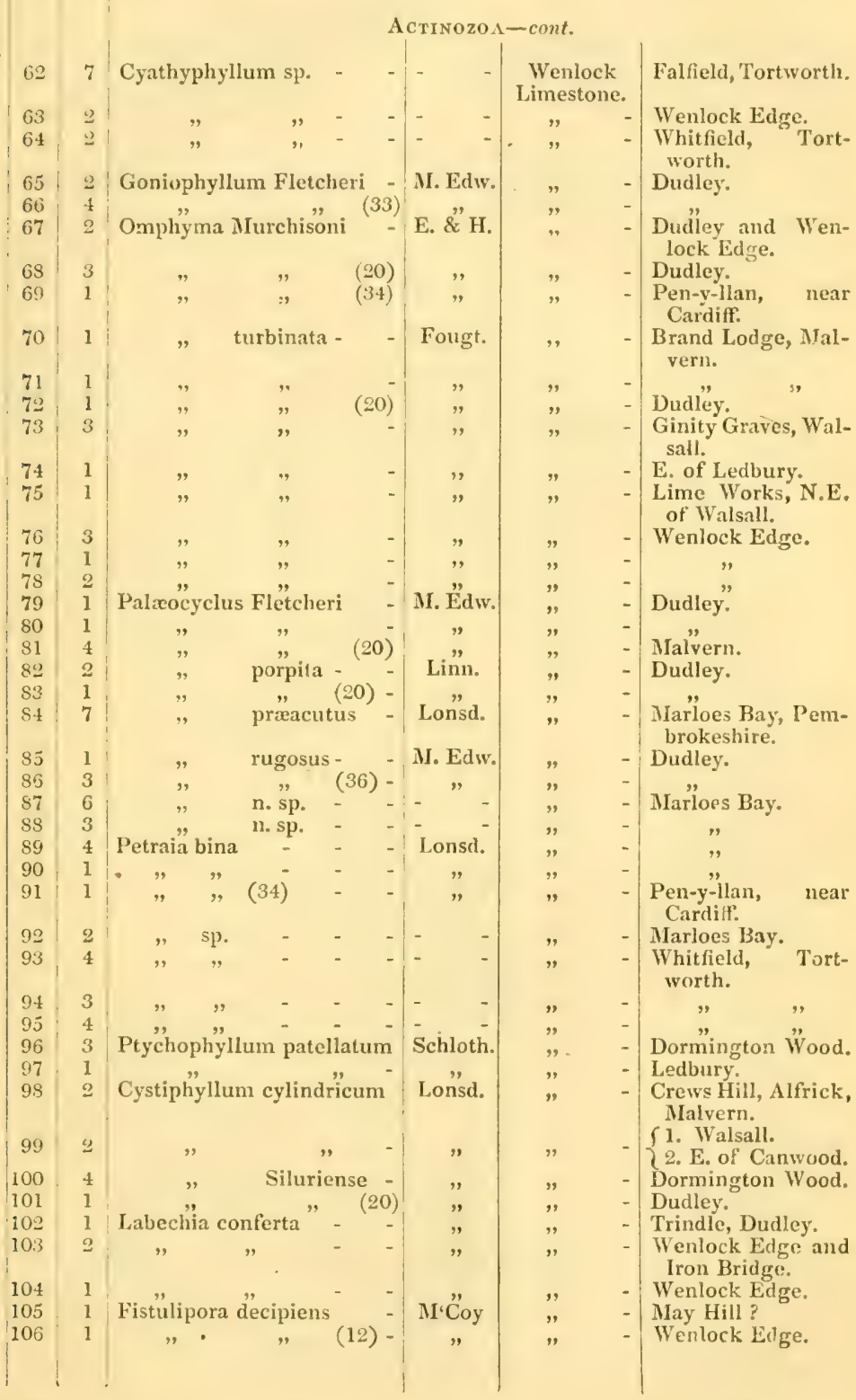




\begin{tabular}{|c|c|c|c|c|c|c|}
\hline 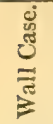 & 造 & $\begin{array}{l}\dot{\tilde{J}} \\
\text { हूँ } \\
\text { के } \\
\text { के }\end{array}$ & Name. & $\begin{array}{l}\text { Author } \\
\text { of } \\
\text { Species. }\end{array}$ & Formation. & Locality. \\
\hline
\end{tabular}

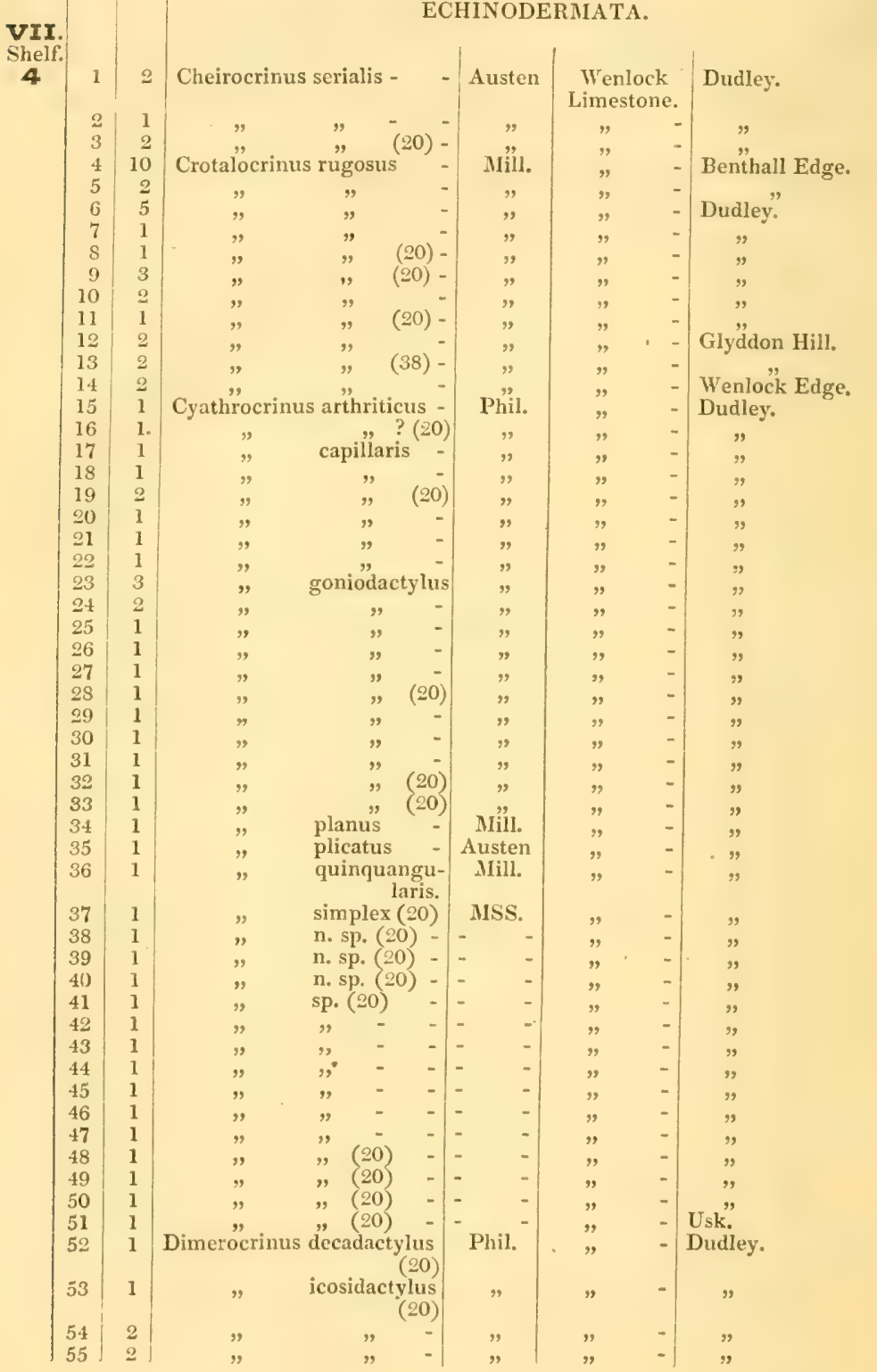




\begin{tabular}{|c|c|c|c|c|c|c|}
\hline 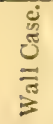 & 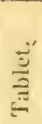 & $\begin{array}{l}\text { हू. } \\
\text { हू } \\
\text { कू } \\
\text { के }\end{array}$ & Name. & $\begin{array}{l}\text { Author } \\
\text { of } \\
\text { Species. }\end{array}$ & Formation. & Locality. \\
\hline
\end{tabular}

VII.

ECHINODERMATA-cont.

Shelf.
4

\begin{tabular}{l|l|l|l|l|l|l|}
\hline 4 & 56 & 1 & Dimerocrinus icosidactylus & Phil. & $\begin{array}{c}\text { Wenlock } \\
\text { Limestone. }\end{array}$ & Dudley.
\end{tabular}

\begin{tabular}{ccccc|c|c|c}
57 & 1 & & & & & Limestone. \\
58 & 2 & $"$ & & - & $"$ & $"$ & -
\end{tabular}

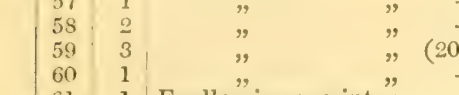

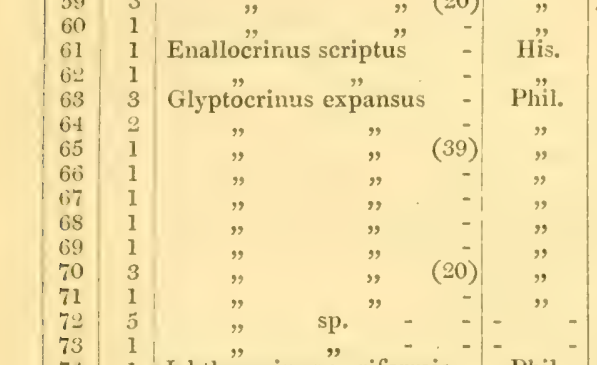

\begin{tabular}{|l|l|l|l|l}
74 & 1 & Ichthyocrinus pyriformis - & Phil. \\
75 & 1 & -
\end{tabular}

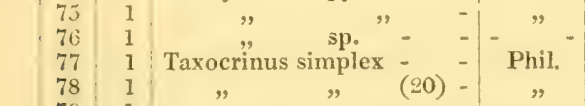

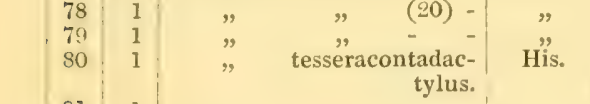

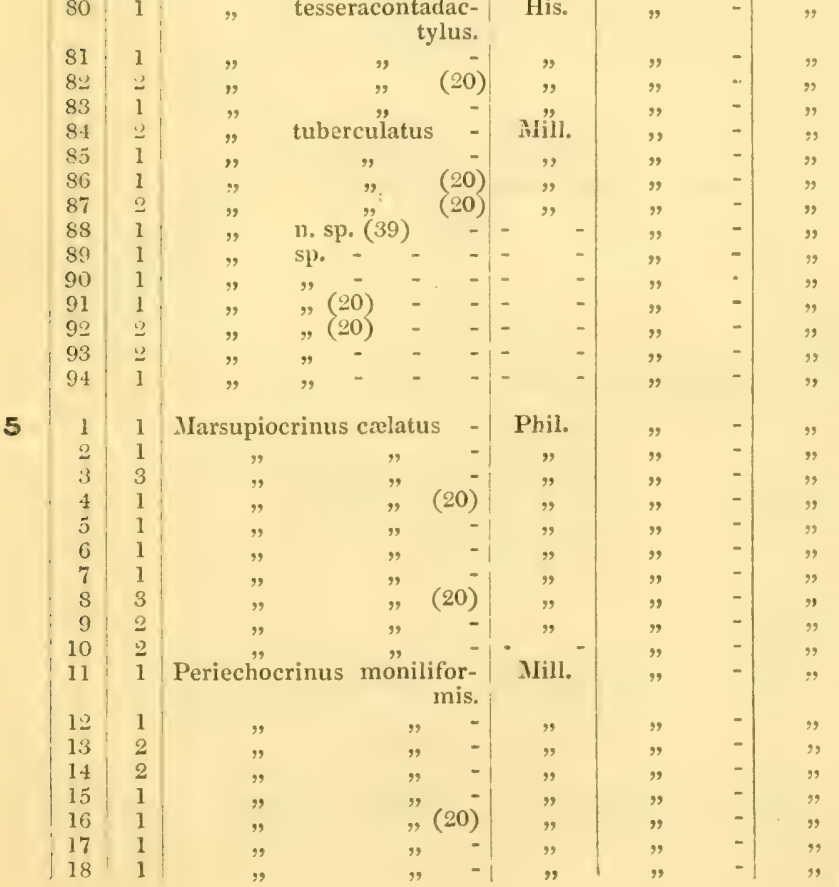




\begin{tabular}{|c|c|c|c|c|c|c|c|}
\hline 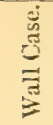 & 苞 & 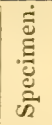 & Name. & " & $\begin{array}{c}\text { Author } \\
\text { of } \\
\text { Species. }\end{array}$ & Formation. & Locality. \\
\hline
\end{tabular}

\section{VII.}

5

\begin{tabular}{|c|c|c|c|c|}
\hline 19 & 1 & $\begin{array}{r}\text { Periechocrinus monilfo } \\
\\
\text { mis }(20\end{array}$ & & Mill. \\
\hline 80 & 1 & $"$ & - & " \\
\hline & 1 & ,? (2 & 20) & , \\
\hline & 1 & n. sp. (20) & & - \\
\hline & 1 &, & - & - \\
\hline & 2 & " $\quad(20)$ & & - \\
\hline & 2 & ", & - & - \\
\hline & 1 & $"$ & -1 & - \\
\hline & 1 & " & - & - \\
\hline & I & sp. $(20)$ & - & - \\
\hline 29 & 1 & $" \quad-$ & - & - \\
\hline 30 & 1 & " $"$ & - & \\
\hline & 1 & Platycrinus retiarius & - & Phil. \\
\hline 39 & 1 & $(20)$ & - & " \\
\hline & 1 & $(20)$ & - & " \\
\hline $34-2$ & 2 & $(20)$ & - & " \\
\hline 36 & 2 & " & - & " \\
\hline 36 & 4 & Eucalyptocrinus decorus & & " \\
\hline 37 & I & " $\quad(40$ & & " \\
\hline & 2 & $" \quad(20$ & & " \\
\hline & 2 & Picoring & & De Kon \\
\hline & 4 & Pisocrints pilula (20) & - & De Kon. \\
\hline & 3 & Eugeniacrinites? laciniatu & & $=$ \\
\hline & 1 & Apiocystites pentrematoid & & Forbes \\
\hline
\end{tabular}




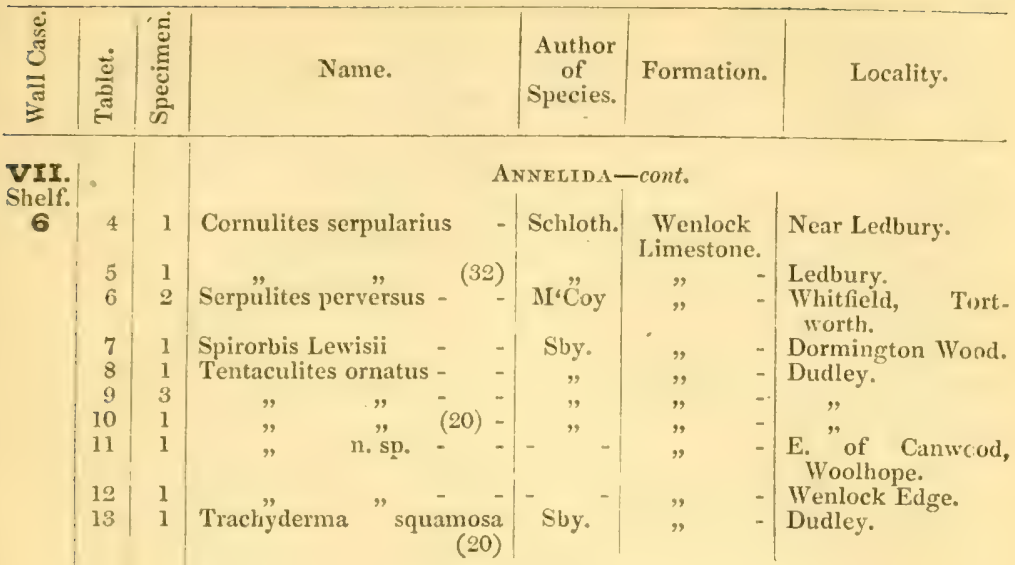

Flat

\section{CRUSTACEA.}

Phacops caudatus

$$
\begin{aligned}
& \text { " } \\
& " \\
& \text { " } \\
& " \\
& \text { " } \\
& \text {, } \\
& \text {," } \\
& \text {, }
\end{aligned}
$$

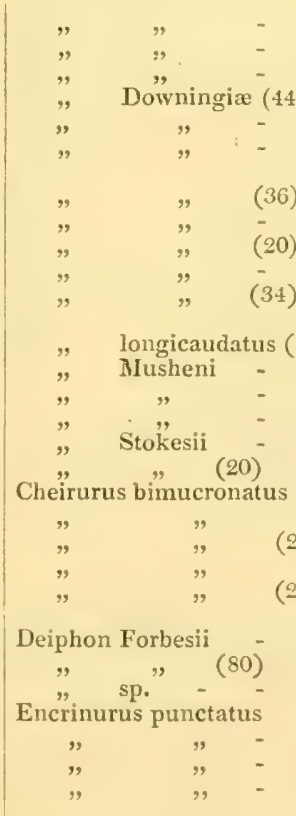

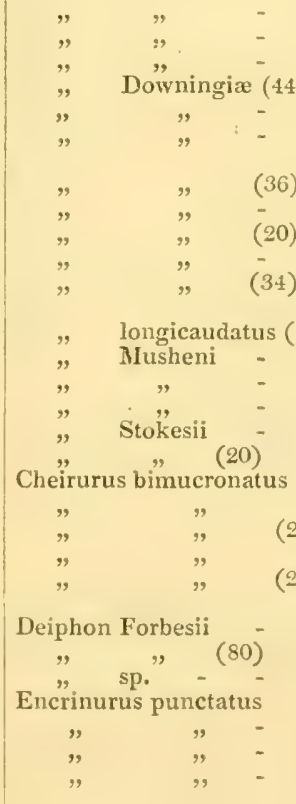

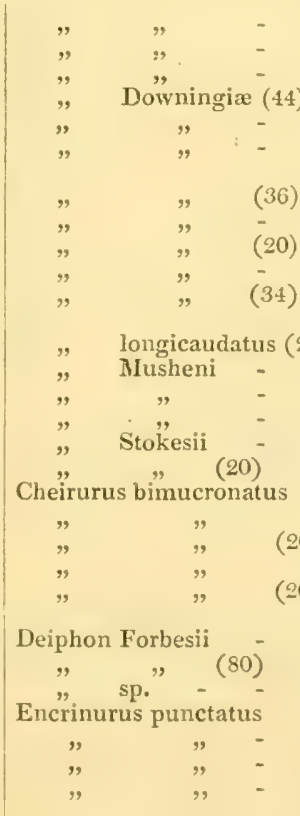$$
10
$$$$
\begin{aligned}
& 11 \\
& 12
\end{aligned}
$$$$
13
$$$$
13 a
$$$$
1
$$$$
16
$$$$
18
$$$$
18
$$$$
19
$$$$
21
$$$$
29
$$$$
23
$$$$
25
$$$$
\mid \begin{aligned}
& 25 \\
& 26 \\
& 27
\end{aligned}
$$$$
+28
$$$$
29
$$$$
\begin{array}{|l|}
30 \\
31
\end{array}
$$$$
32
$$$$
\begin{aligned}
& 33 \\
& 34
\end{aligned}
$$$$
\begin{aligned}
& 34 \\
& 35
\end{aligned}
$$

- Brün. Wenlock | Limestone. $\begin{array}{ll}" & - \\ " & - \\ " & - \\ " & - \\ & -\end{array}$

- Schucknall Hill, Woolhope.

- Dudley. Malvern.

- "

- E. of Upper Hall, Ledbury.

Dudley.

",

Led"bury.

Pen-y-llan, near Cardiff.

Malvern.

- Walsall.

Dudley.

Walsall.

Dudley.

- ,

-

- Dormington.

Dranhill Quarry, near Malvern.

- Dudley.

- $\begin{gathered}\text { " } " \\ \text { - } \\ \text { - Walsall. }\end{gathered}$ 


\begin{tabular}{|c|c|c|c|c|c|c|}
\hline 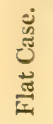 & 离 & $\begin{array}{l}\text { हूँ } \\
\text { हूँ } \\
\text { के }\end{array}$ & Name. & $\begin{array}{c}\text { Author } \\
\text { of } \\
\text { Species. }\end{array}$ & Formation. & Locality. \\
\hline
\end{tabular}

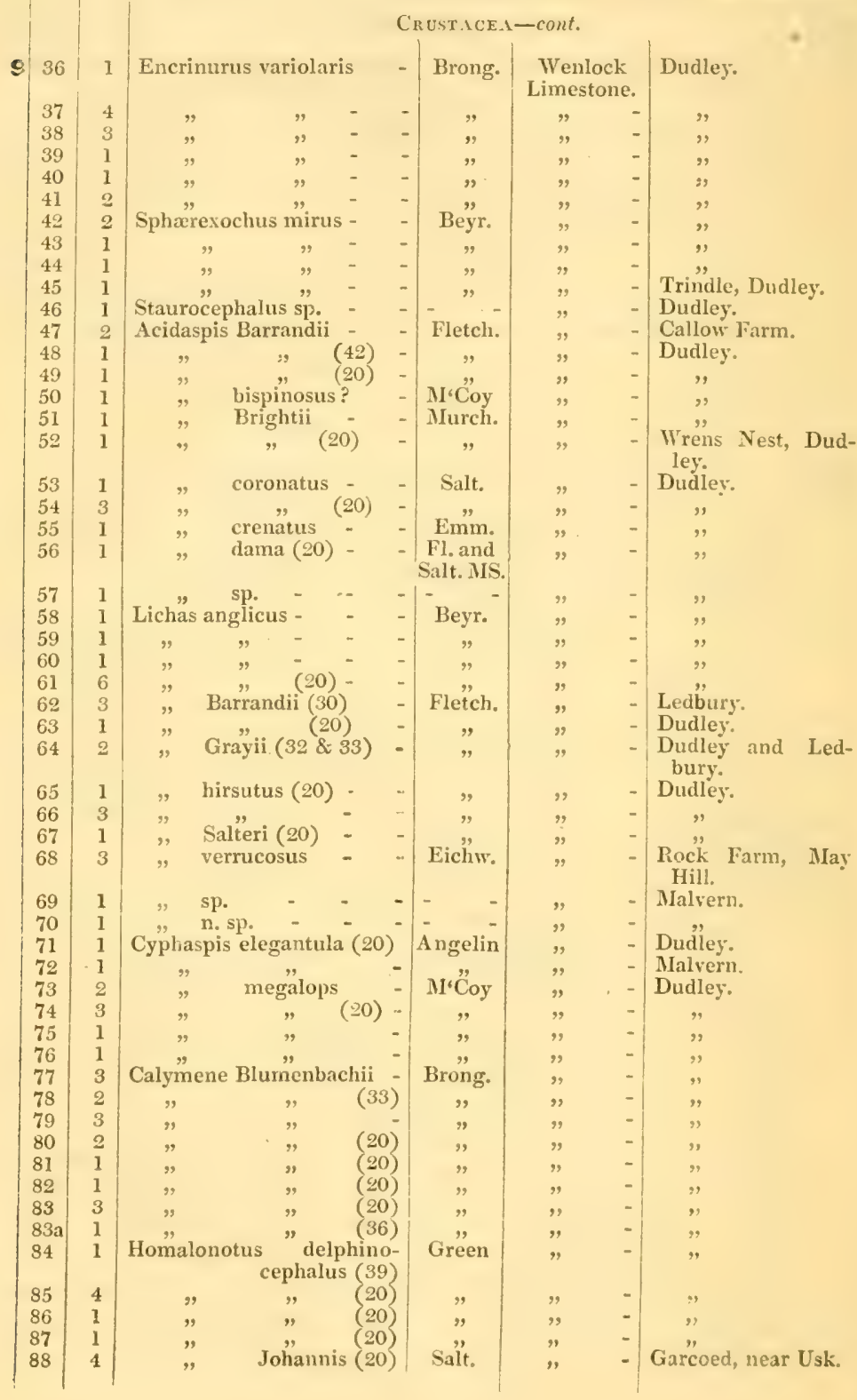




\begin{tabular}{|c|c|c|c|c|c|c|}
\hline 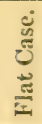 & 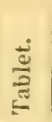 & 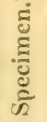 & Name. & $\begin{array}{l}\text { Author } \\
\text { of } \\
\text { Species. }\end{array}$ & Formation. & Locality. \\
\hline
\end{tabular}

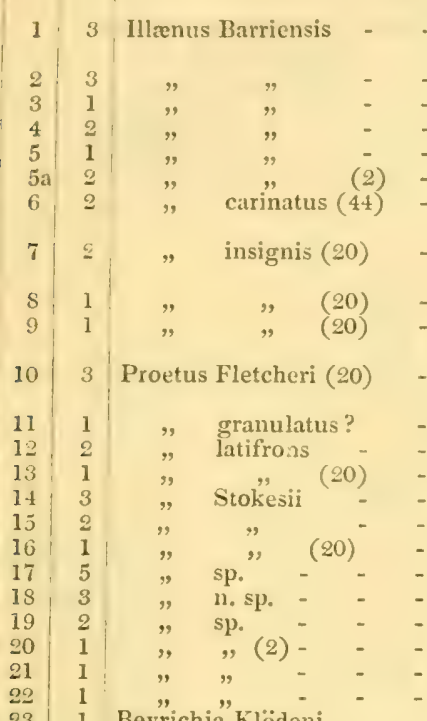

Beyrichia Klödeni

" " (20) thica, His.

261 Eurypterus punctatus (20)

27

$27 \mathrm{a}^{\prime}$

29

Drawer.

19

Crustaces-cont.

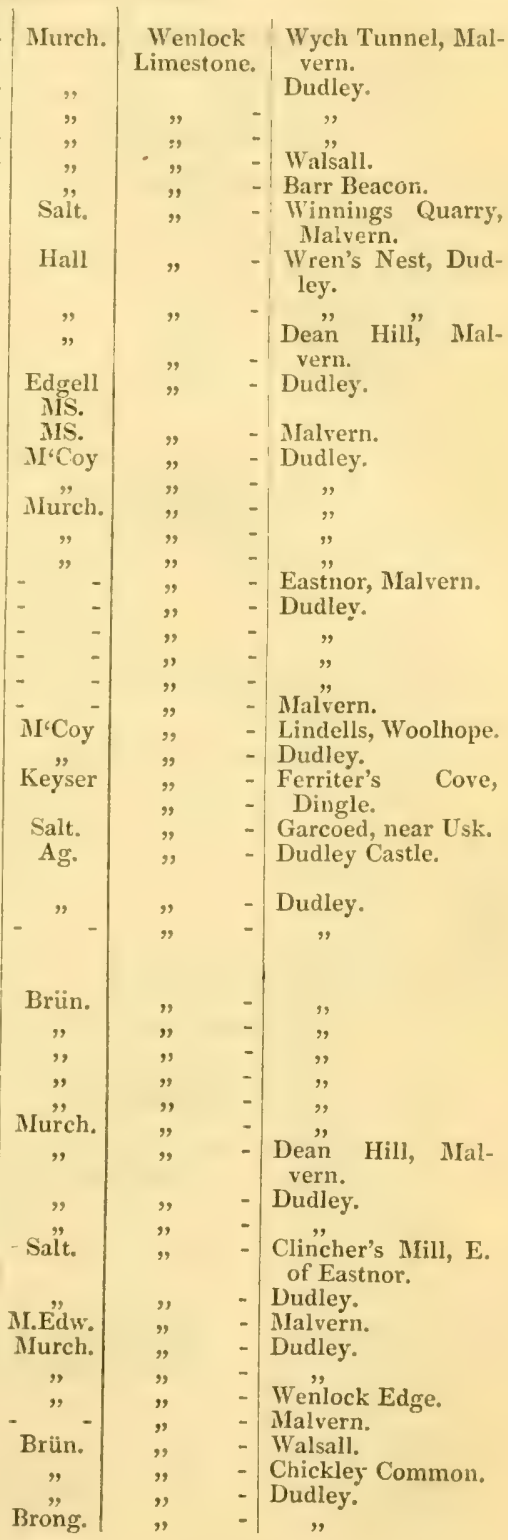




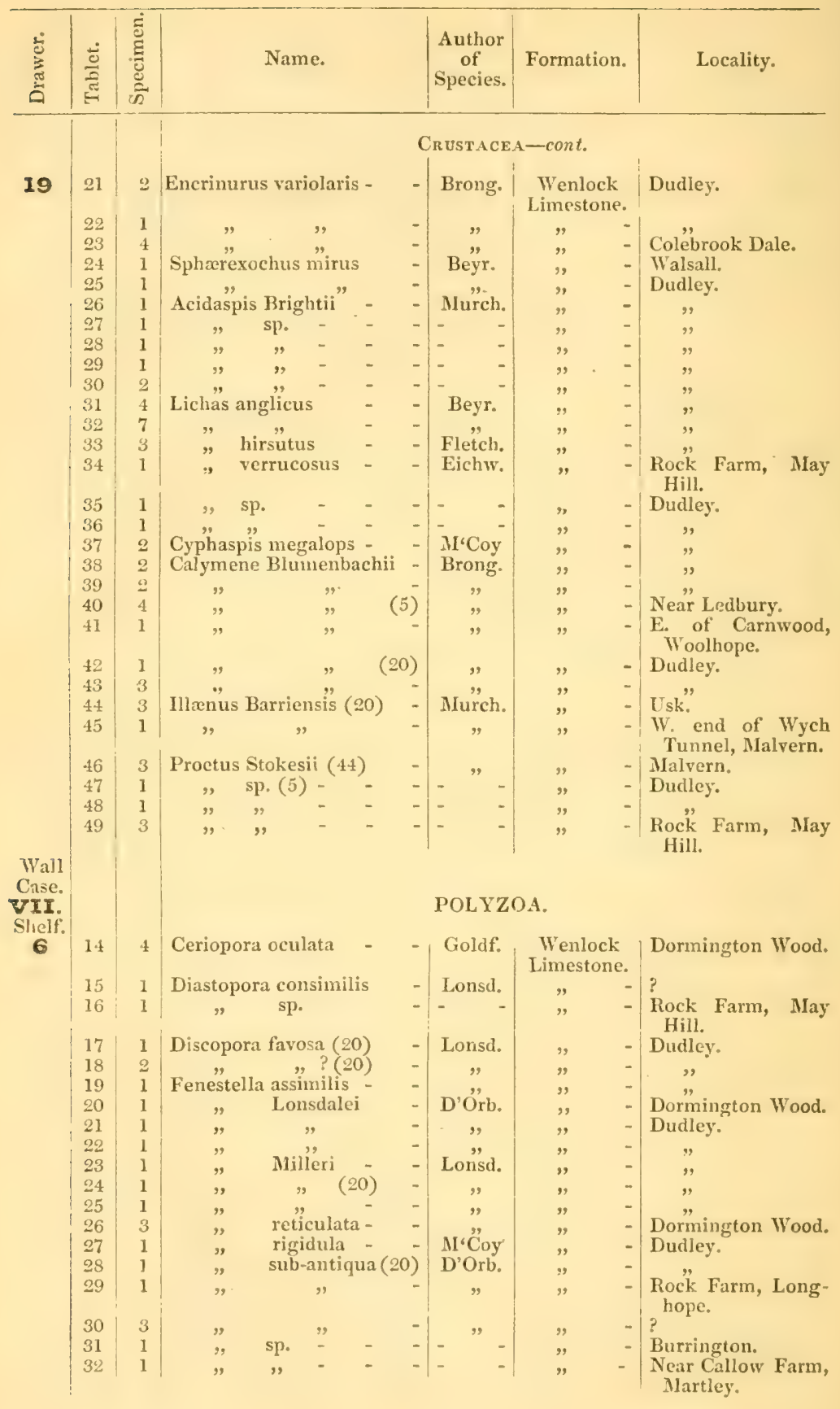




\begin{tabular}{|c|c|c|c|c|c|c|}
\hline 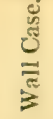 & है & 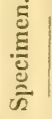 & Name. & $\begin{array}{l}\text { Author } \\
\text { of } \\
\text { Species. }\end{array}$ & Formation. & Locality. \\
\hline
\end{tabular}

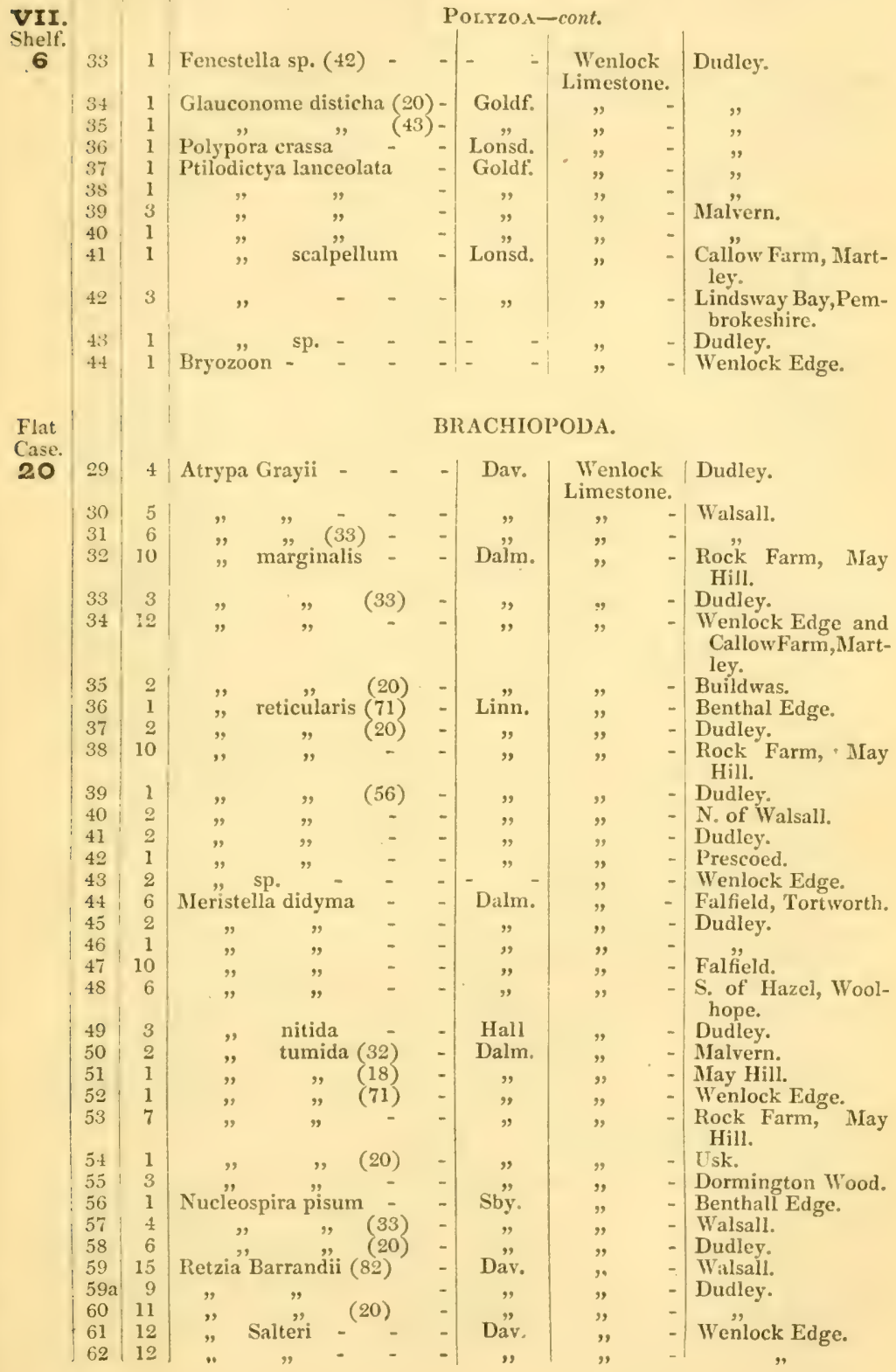




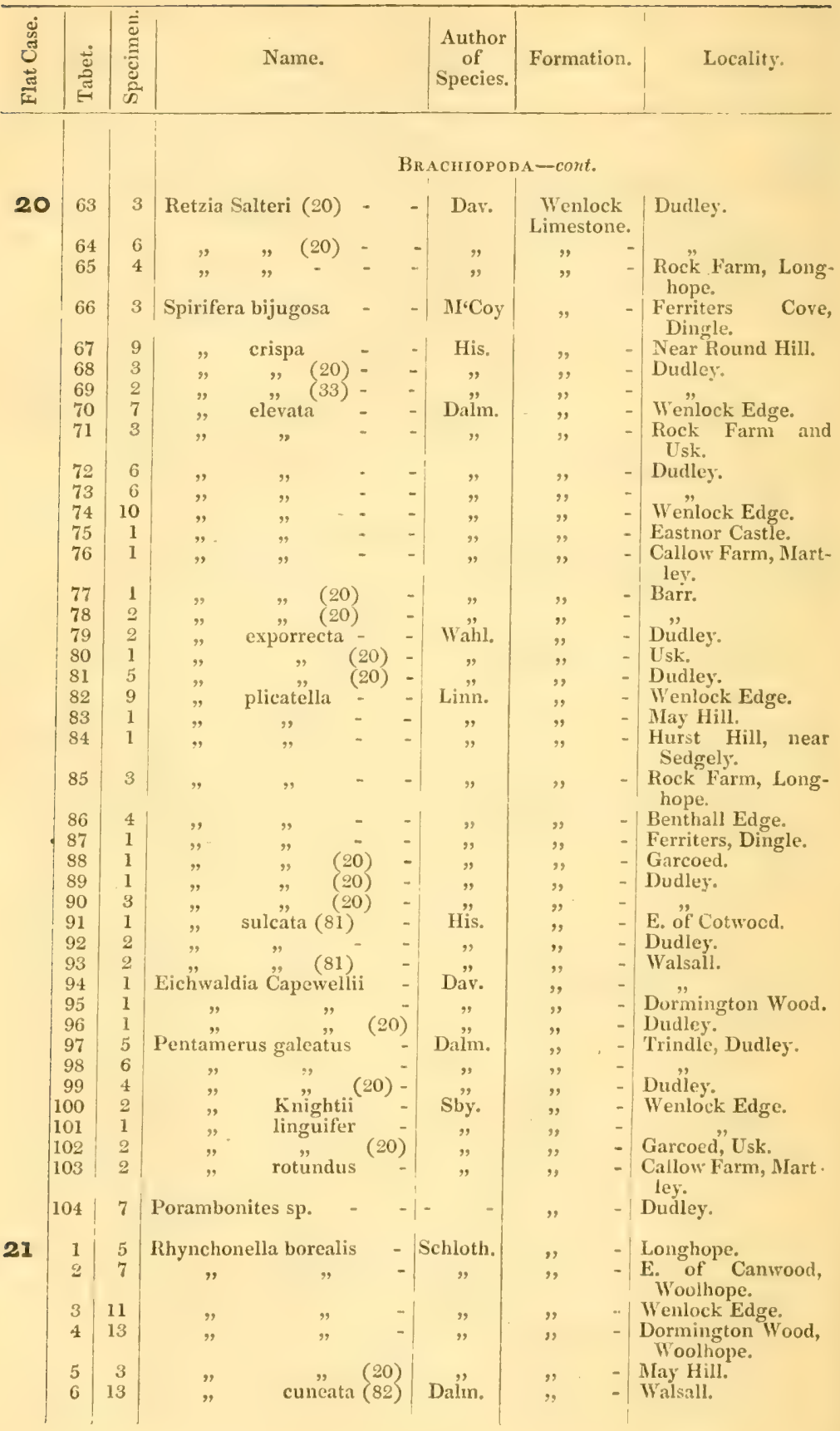




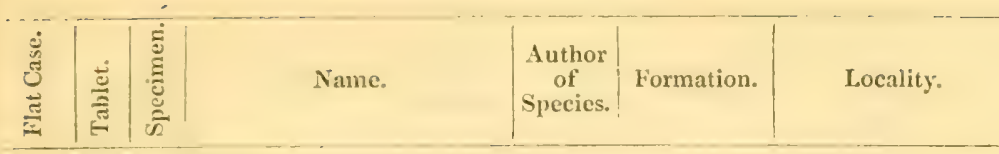

\section{Br.CHOron-cont.}

21

\begin{tabular}{|l|l|l|l|}
7 & 3 & Rhynchonella cuneata (20) & Dalm. \\
\hline 5 & 1 &
\end{tabular}

(

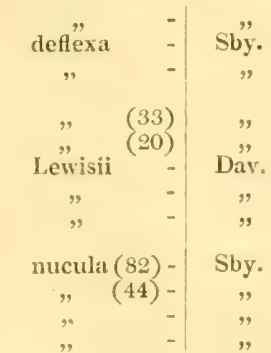

\section{Salteri -} Stricklandi (32) ", (20) Tilsoni (82)

\begin{tabular}{|r|r|}
24 & 2 \\
25 & 11
\end{tabular}
$|26| 5 \mid$ \begin{tabular}{|l|l|l|}
27 & 4 & $\quad "$ \\
28 & 6 & $\quad "$ \\
29 & 6 & $\quad "$ \\
30 & 1 & $\quad "$
\end{tabular} \begin{tabular}{|l|l|}
31 & 10 \\
32 & 11
\end{tabular} \begin{tabular}{r|r|}
32 & 11 \\
33 & 9
\end{tabular}
Wenlock Limestone.

\begin{tabular}{ll|l}
, & - & N. of Canwood. \\
, & - & Walsall. \\
, & - & ley. and Dud- \\
, & - & Dudley.
\end{tabular}

Trindle, Dudley.

- The Rock, May Hill.

- Walsall.

- Malvern.

- Botvyle, Caradoc.

- Hope End Park, Tottenham.

- Dudley.

- Hobbs, Longhope.

- Malvern.

- Nash Scar, Presteign.

- Walsall.

- Whitfield, Tortworth.

- Scar Lime Works, Onibury.

- Dudley.

- Eastnor gravel pit.

- Walsall.

- Dudley.

- Wenlock Edge,

- Storridge, Malvern.

- Walsall.

Wahl.

- Schloth.

- Linn.

$-1 \quad n$

Dar.

Dalm.

Lindst.

",

,

Hili).

(Lincoln

Rock Farm, May Hill.

- Wenlock Edge.

- Walsall.

- Malvern.

- Dormington, Woolhope.

- Garcoed, Usk.

- Whitfield, Tortworth.

- Dudley.

- S.E. of Carnwood.

- Rock Farm, Longhope.

- Garcoed, Usk.

- Dudley.

- Dormington Woxd.

- Dudley.

- Wenlock Edge.

- Walsall.

- Dudley. 


\begin{tabular}{|c|c|c|c|c|c|c|}
\hline 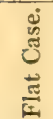 & 造 & 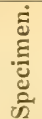 & Name. & $\begin{array}{l}\text { Author } \\
\text { of } \\
\text { Species. }\end{array}$ & Formation. & Locality. \\
\hline
\end{tabular}

\section{BRACHIOPODA-cont.}

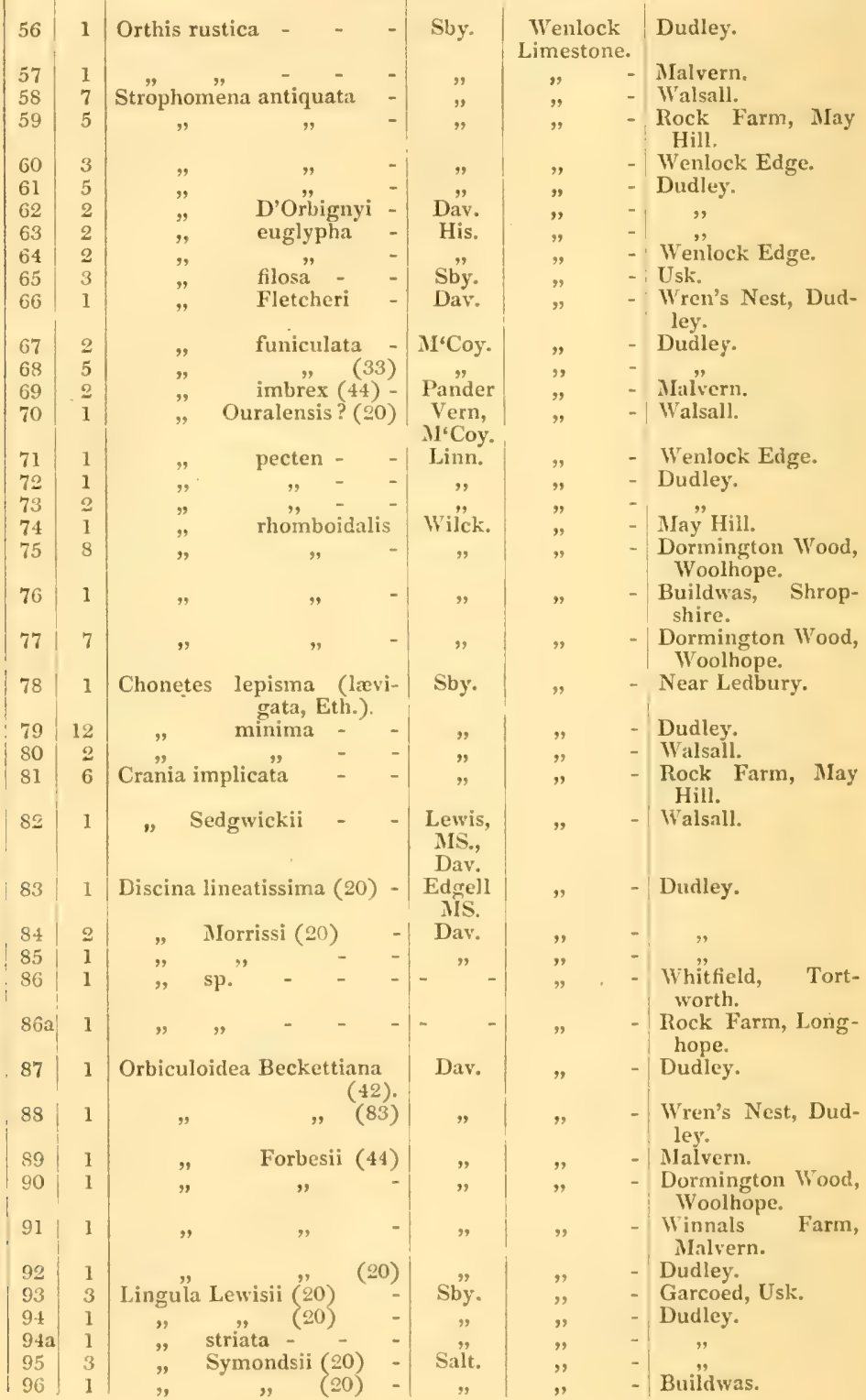




\begin{tabular}{|c|c|c|c|c|c|c|}
\hline 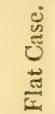 & 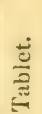 & 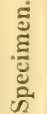 & Name. & $\begin{array}{c}\text { Author } \\
\text { of } \\
\text { Species. }\end{array}$ & Formation. & Locality. \\
\hline
\end{tabular}

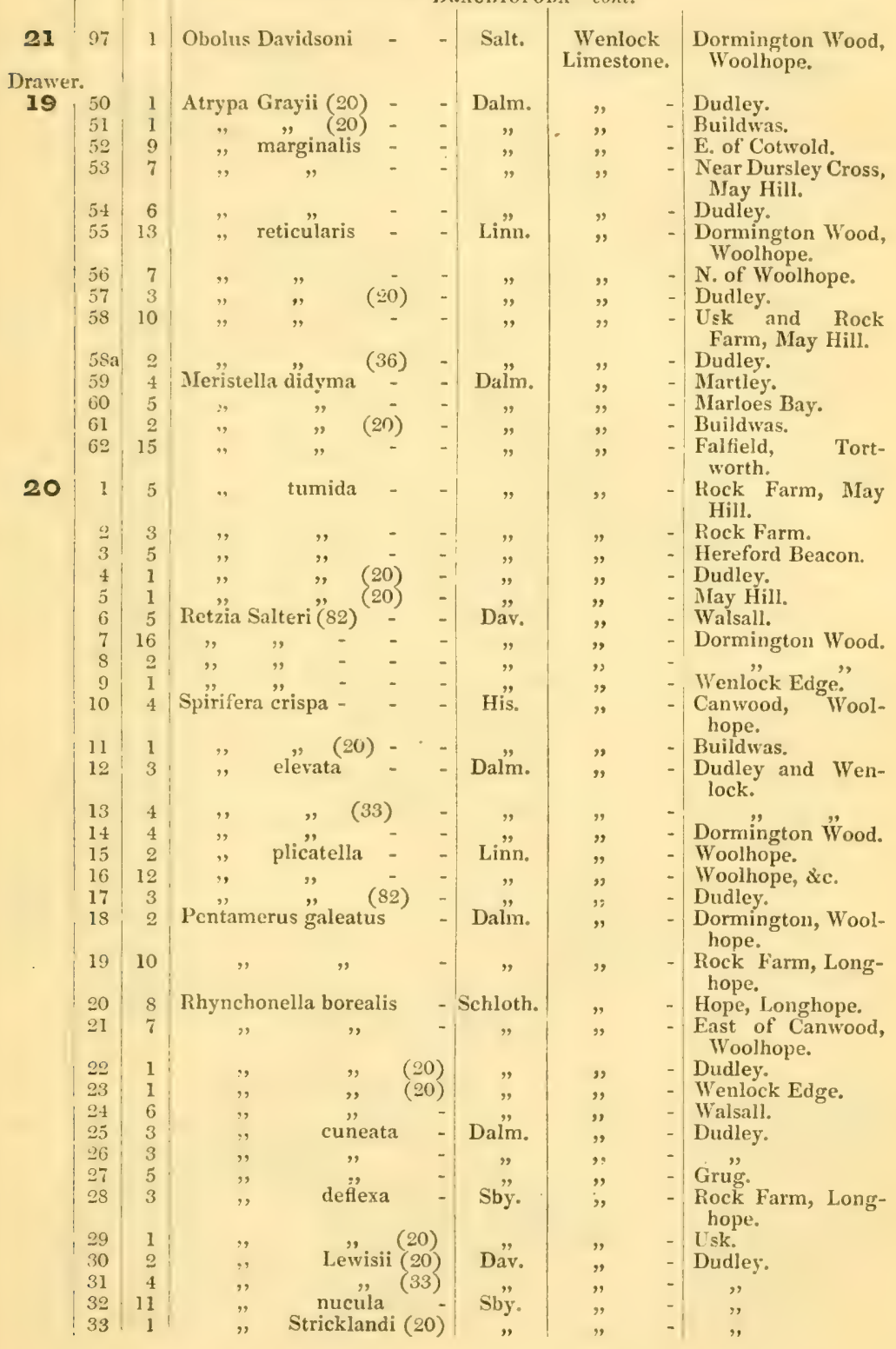




\begin{tabular}{|c|c|c|c|c|c|}
\hline 悹 & 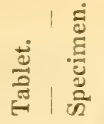 & Name. & $\begin{array}{l}\text { Author } \\
\text { of } \\
\text { Species. }\end{array}$ & Formation. & Locality. \\
\hline
\end{tabular}

BrACHIOPODA-cont.

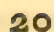

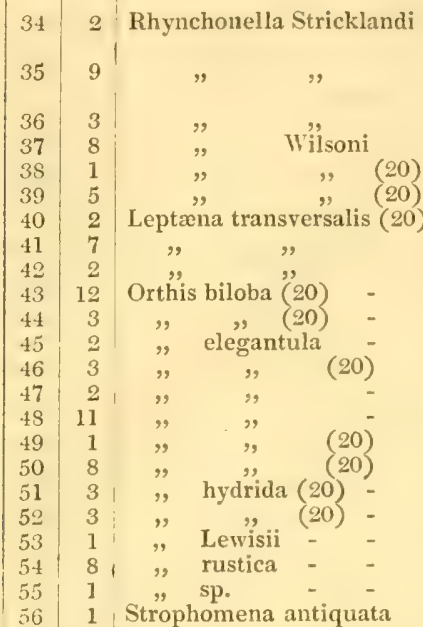

, (20)

D'Orbignyi euglypha

"ilosa

(20)

funiculata $(20)$

imbrex -

, (44) pecten (20) rhomboidalis

Flat

Case.

22

\begin{tabular}{|c|c|c|c|}
\hline Sby. & \multicolumn{2}{|c|}{$\begin{array}{l}\text { Wenlock } \\
\text { Limestone. }\end{array}$} & Walsall. \\
\hline " & " & 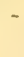 & $\begin{array}{l}\text { East of Canwood, } \\
\text { Woolhope. }\end{array}$ \\
\hline$"$ & $"$ & - & Dudley. \\
\hline$"$ & " & - & Clinchers Mill. \\
\hline$"$ & " & - & Dudley. \\
\hline Wahl. & $"$ & - & Usk." \\
\hline$"$ & " & - & Dudley. \\
\hline Ling & ", & - & \\
\hline Linn. & " & - & $\begin{array}{l}\text { Wenlock Edge. } \\
\text { Dudley. }\end{array}$ \\
\hline Dalm. & $"$ & - & Dormington Wood. \\
\hline " & " & - & \\
\hline$"$ & 将 & - & $"$ \\
\hline " & $"$ & - & Usk." \\
\hline$"$ & , & & Garcoed, Usk. \\
\hline Sby. & $"$ & & Dudley. \\
\hline Dav" & " & 一 & Buildwas. \\
\hline $\begin{array}{l}\text { Dav. } \\
\text { Sby. }\end{array}$ & " & - & Walsall. \\
\hline soy. & $"$ & - & Wenlock Edge. \\
\hline Sby. & ", & - & Dudley. \\
\hline$"$ & $"$ & & Dormington Wood. \\
\hline D" & $\eta$ & & Dudley. \\
\hline $\begin{array}{l}\text { Dav. } \\
\text { His. }\end{array}$ & $"$ & 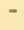 & Walsall. \\
\hline $\begin{array}{l}\text { His. } \\
\text {,y }\end{array}$ & ", & 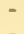 & Dudley. \\
\hline S by. & " & & Dudley. \\
\hline M'Coy & " & - & $"$ \\
\hline Pand. & $"$ & - & " \\
\hline Linn & 9 & & May Hill. \\
\hline & " & 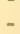 & Dudley. \\
\hline Wilck. & 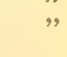 & - & $\begin{array}{l}\text { Beacon Hill Quarry, } \\
\text { near Sedgeley. }\end{array}$ \\
\hline " & $"$ & - & $\begin{array}{l}\text { The Rock, May } \\
\text { Hill. }\end{array}$ \\
\hline$"$ & " & & Dormington Wood. \\
\hline ", & $"$ & - & $\begin{array}{l}\text { Woodgreen, Long- } \\
\text { hope. }\end{array}$ \\
\hline$"$ & $"$ & & $\begin{array}{l}\text { Linley Lime Kilns, } \\
\text { Walsall. }\end{array}$ \\
\hline " & $"$ & - & Dudley \\
\hline " & $"$ & & , \\
\hline
\end{tabular}

\section{LAMELLIBRANCHIATA.}

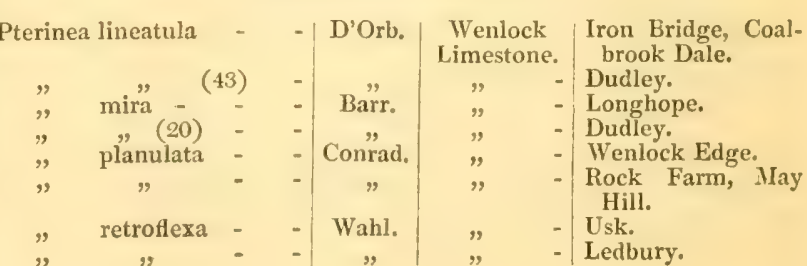




\begin{tabular}{|c|c|c|c|c|c|c|}
\hline 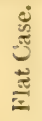 & $\stackrel{\frac{\overrightarrow{0}}{5}}{\stackrel{5}{\pi}}$ & 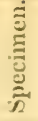 & Name. & $\begin{array}{l}\text { Author } \\
\text { of } \\
\text { Species. }\end{array}$ & Formation. & Locality. \\
\hline
\end{tabular}

\section{AMELLIBRANCHIATA-cont.}

22

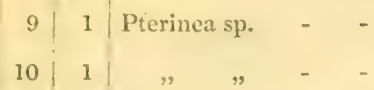

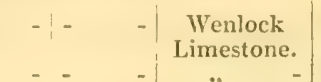

\begin{tabular}{l|l|ll}
11 & 1 & $\Rightarrow$ &,$(20)$
\end{tabular}

$1212, ",(20)$

131 Modiolopsis antiqua -

$15 \pm$ Cardiola fibrosa - - - Sby.

163 " striata - - - "

174 Pleurorhynchus aquicos- Phil.

tatus.

18 Goniophora cymbxformis -

\begin{tabular}{l|l|l}
19 & 1 & G' \\
20 & 1 & Grammysia cingulata (44)
\end{tabular}

\begin{tabular}{l|l|l}
20 & 1 & Grammysia cingulata (44) \\
21 & 1 &,,
\end{tabular}

22

1

Orthonota teres (20) -

Ctenodonta anglica -

24

25

Drawer.

\section{GASTEROPODA.}

Crew's Hill, Alfrick. Near Callow Farm, Martley.

May Hill.

Wenluck Edge.

Rock Farm, Longhope.

Dudley.

Wenlock Edge.

Dudley.

Malvern.

Dud̉ley.

Rock Farm, Longhope.

Dudley.

Malvern.

Dudley.

May" Hill.

Alfrick.

Usk.

Dormington Wood.

Pen-y-llan, near Cardiff?

Falfield, Tortworth.

Dudley.

Wenlock Edge.

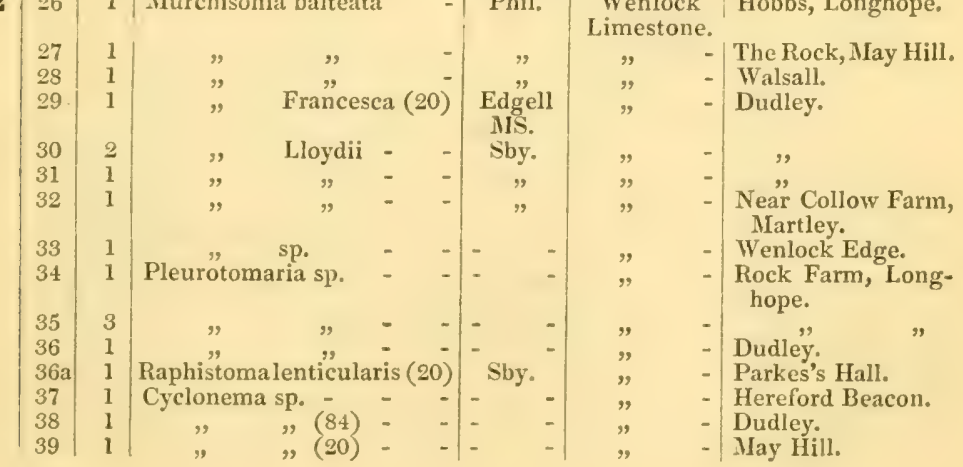




\begin{tabular}{|c|c|c|c|c|c|c|}
\hline 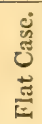 & 芯 & 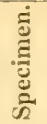 & Name. & $\begin{array}{l}\text { Author } \\
\text { of } \\
\text { Species. }\end{array}$ & Formation. & Locality. \\
\hline
\end{tabular}

22

\section{Drawer.}

\section{GASTEROPODA-cont.}

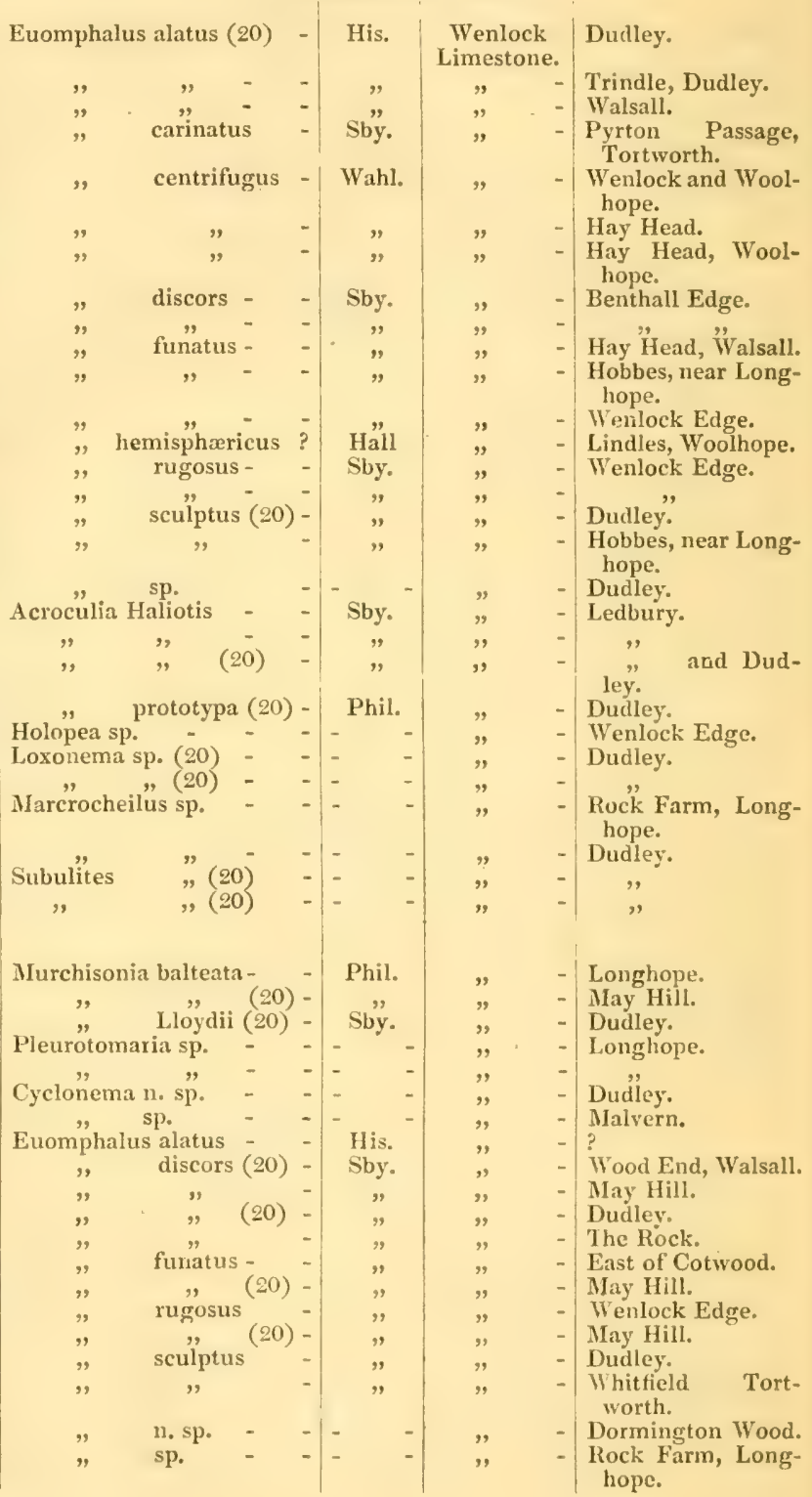




\begin{tabular}{|c|c|c|c|c|c|c|}
\hline$\frac{2}{2}$ & है & 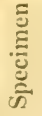 & Name. & $\begin{array}{c}\text { Author } \\
\text { of } \\
\text { Species. }\end{array}$ & Formation. & Locality. \\
\hline
\end{tabular}

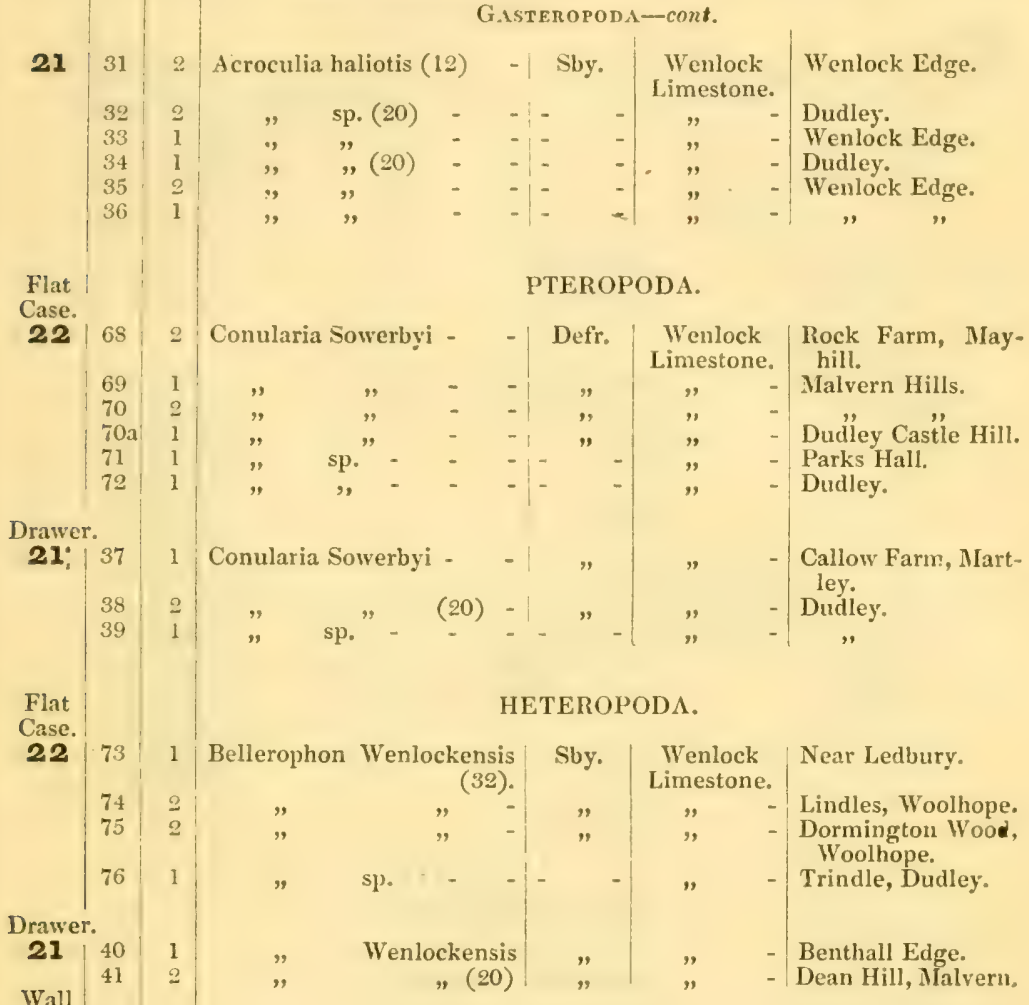

\section{CEPHALOPODA.}

$$
\begin{aligned}
& \text { Lituites articulatus - - Sby. Wenlock Wenlock Edge. } \\
& \text { Limestone. } \\
& \text { - Dudley. } \\
& \text { - Dean Hill, Mal- } \\
& \text { vern. } \\
& \text { I'sk. } \\
& \text { Malvern. } \\
& \text { - Gar"cued, near L'sk. } \\
& \text { - Dormington Ẅood. } \\
& \text { - Dudley. } \\
& \text { - Garcoed, near Usk. } \\
& \text { - Hag Head. } \\
& \text { - Hurst Hill, Sedg- } \\
& \text { ley. } \\
& \text { Ledbury. } \\
& \text { - Malvern. }
\end{aligned}
$$




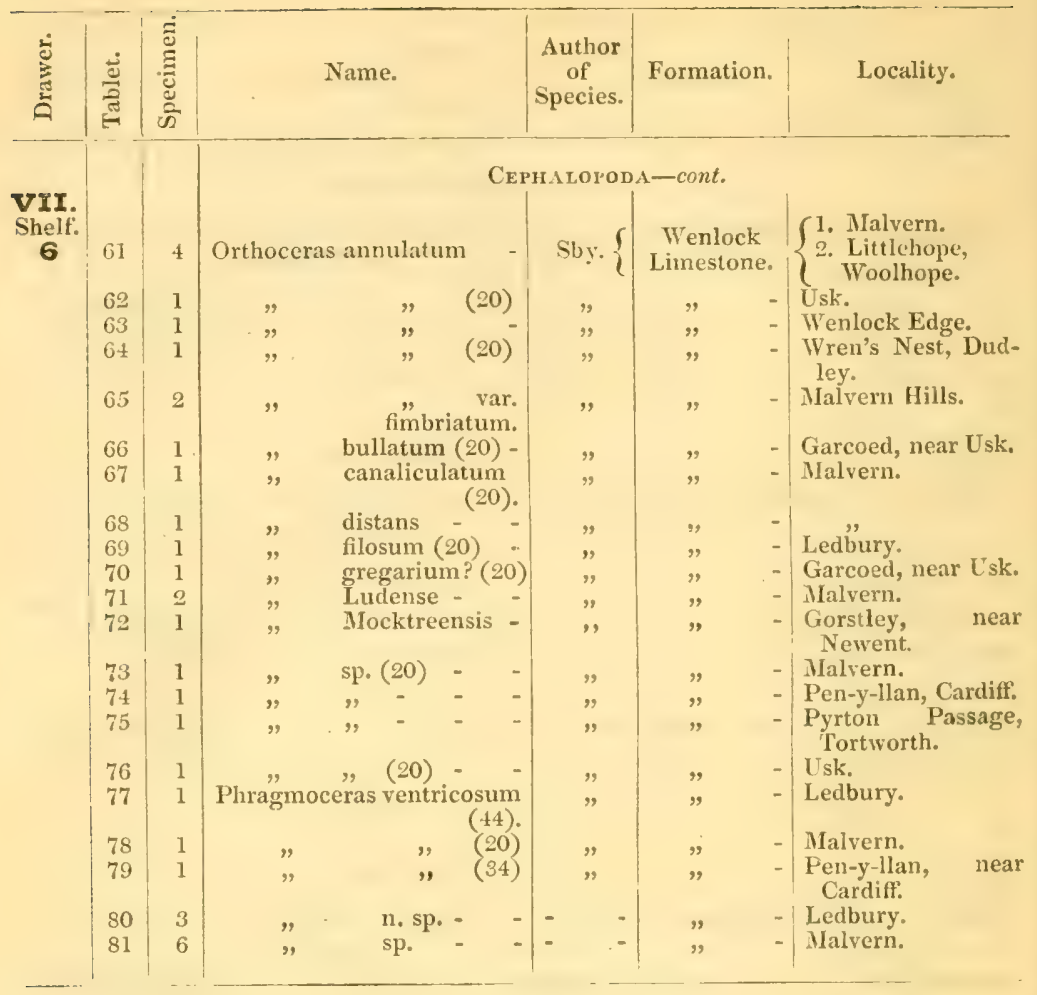

UPPER SILURIAN-cont.

IOWIR IUDIOW.

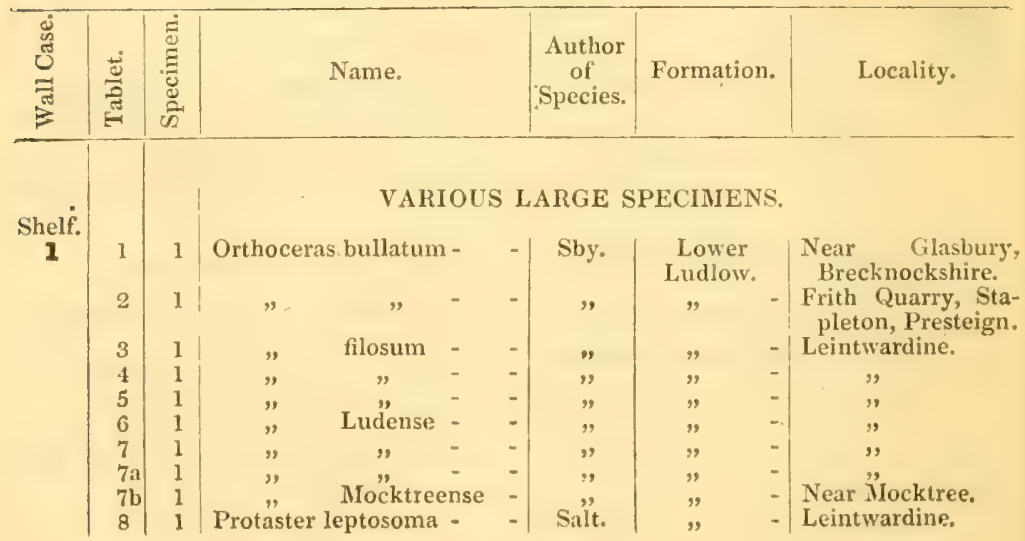




\begin{tabular}{|c|c|c|c|c|c|c|}
\hline $\begin{array}{l}0 \\
0 \\
0 \\
0\end{array}$ & $1 \frac{\tilde{E}}{\mathrm{E}}$ & $\frac{\bar{\Xi}}{\stackrel{\Xi}{\Xi}}$ & Name. & $\begin{array}{c}\text { Author } \\
\text { of } \\
\text { Species. }\end{array}$ & Formation. & Locality. \\
\hline
\end{tabular}

I25.

shelt.

2

PLANTA:.

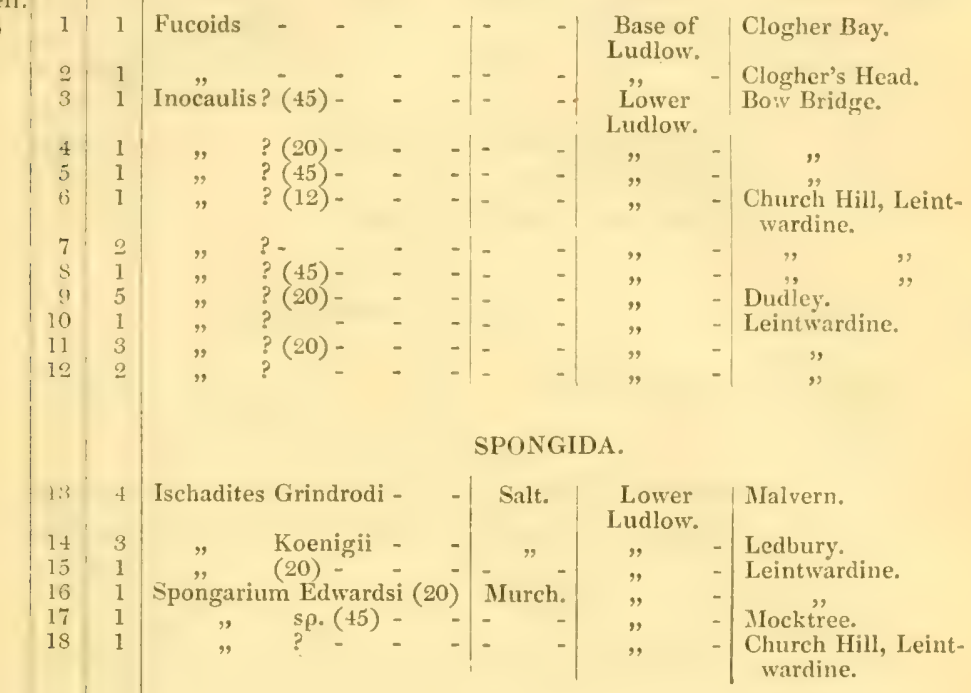

HYDROZOA.

Dendrograptus sp. (45) - -

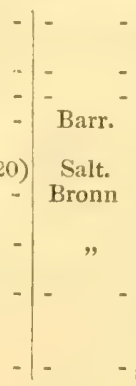

Lower

Bow Bridge.

Ludlow.

Graptolithus col"

$\left(\begin{array}{ll}45) & -\end{array}\right.$

, $\quad$ Flemingii (20) priodon

,

- Leintwardine.

- Vinnal Hill, W. side.

- Leintwardine.

- Cefn Erthan, Llarduvery.

- Vinnal Hill, Lud. low.

- Llanerch Coch, Myddfai Mountain.

- Leintwardine.

ACTINOZOA.

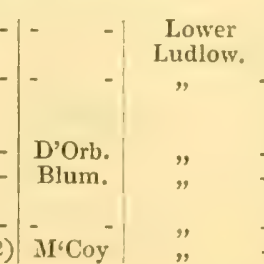

Bow Bridge.

- Base of Croagh, Marhen, Doonquin, Dingle.

Pyrton Passage.

- Coosmore, Clogher Head.

- Pyrton Passage.

- Ludlow. 


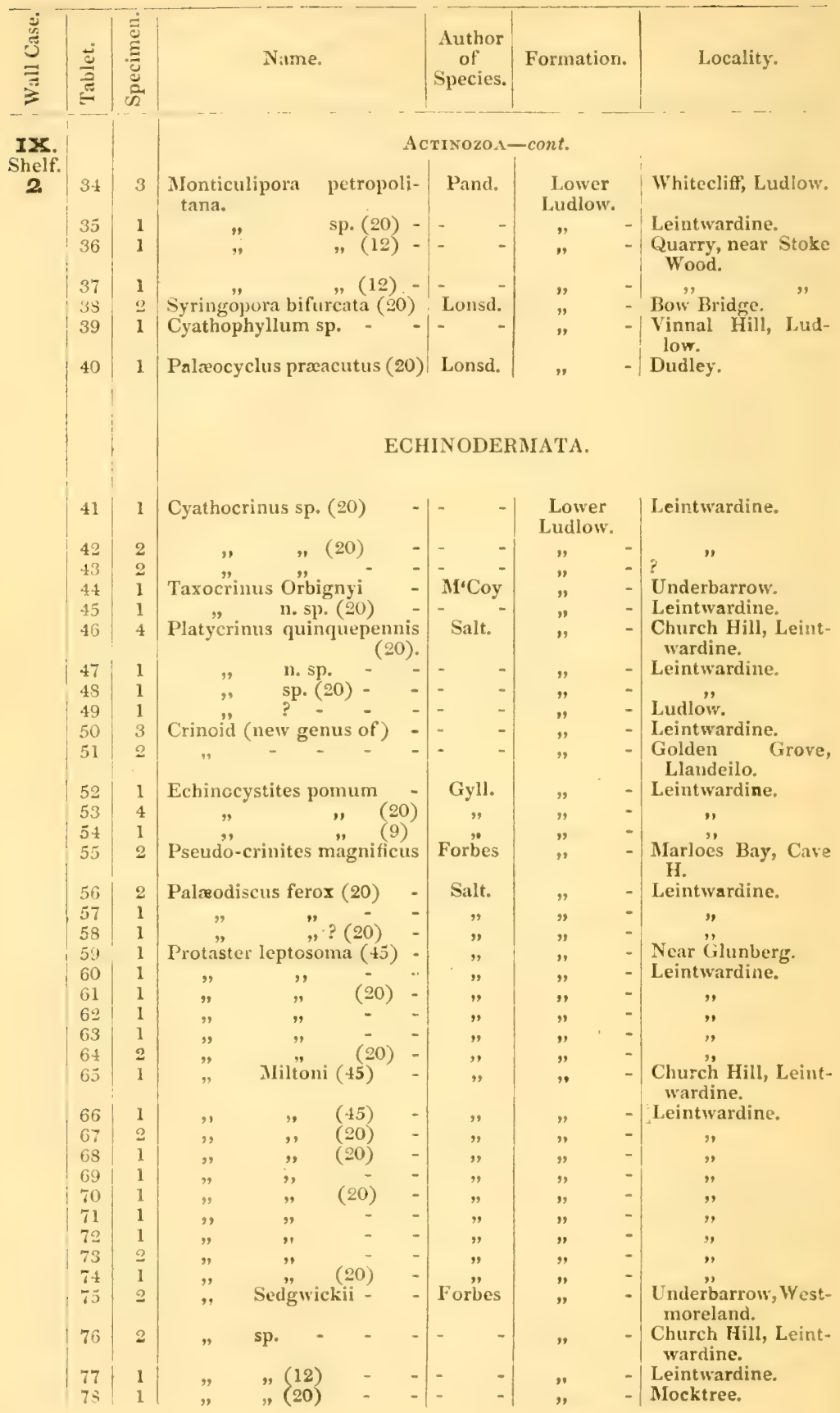




\begin{tabular}{|c|c|c|c|c|c|c|}
\hline$\stackrel{3}{=}$ & 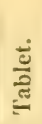 & $\begin{array}{l}\text { हूँ } \\
\text { हूँ } \\
\text { कू }\end{array}$ & Name. & $\begin{array}{l}\text { Author } \\
\text { of } \\
\text { Species. }\end{array}$ & Formation. & Locality. \\
\hline
\end{tabular}

Ix.

Shelf.

3

\begin{tabular}{|c|c|c|c|c|c|c|c|}
\hline \multirow[b]{2}{*}{1} & \multirow[b]{2}{*}{3} & \multicolumn{6}{|c|}{ ECHINODERMATA-cont. } \\
\hline & & Palæaster hirudc & - & Forbes & $\begin{array}{l}\text { Lower } \\
\text { Ludlow: }\end{array}$ & & $\begin{array}{l}\text { Underbarrow, Ken- } \\
\text { dal. }\end{array}$ \\
\hline 2 & 4 & Palzeasterina pri & mava & & , & - & $, \quad, \quad$, \\
\hline 3 & 2 & Palacocoma Colv & ini $-\quad-$ & Salt. & " & - & Leintwardine. \\
\hline 4 & 1 & cygr & ipes (45) - & " & $"$ & - & " \\
\hline 5 & 2 & Mar & stoni - & " & ", & - & $"$ \\
\hline 6 & 1 & " & $(46)-$ & " & $"$ & - & , \\
\hline 7 & 1 & " & $(20)-$ & $"$ & 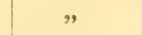 & - &. \\
\hline 8 & 1 & $"$ & - & " & $"$ & - & . \\
\hline 9 & 2 & " & $(20)-$ & , & , & - & . \\
\hline 10 & 1 & $"$ & - & , & , & - & ., \\
\hline $10 \mathrm{a}$ & 1 & " & $(69)-$ & $"$ & " & - & ", \\
\hline 11 & 1 & , & ". - & " & " & - & \\
\hline 12 & 1 & vern & hiformis - & " & $"$ & - & Leintwardine. \\
\hline 13 & 1 & " & $(12)$ & $"$ & $"$ & - & " \\
\hline 14 & 3 & , & $(20)$ & $"$ & $"$ & - & $\begin{array}{l}\text { Mocktree and } \\
\text { Leintwardine }\end{array}$ \\
\hline 15 & l & $"$ & $" \quad(45)$ & "' & $"$ & - & Mocktree. \\
\hline 16 & 1 & " $\quad$ 11. s1 & ) $(20)$ & & $"$ & - & Leintwardine. \\
\hline 17 & 1 & Rhophalocoma & $\begin{array}{l}\text { pyrotech- } \\
\text { nica (45). }\end{array}$ & Salt. & " & - & $"$ \\
\hline $\begin{array}{l}18 \\
19\end{array}$ & $\begin{array}{l}1 \\
3\end{array}$ & Star fish (youn & $(20))^{(45)}$ & $"$ & $"$ & - & $"$ \\
\hline 19 & & Not hish (yot & 7 & 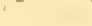 & " & & 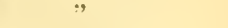 \\
\hline
\end{tabular}

A NNEIIDA.

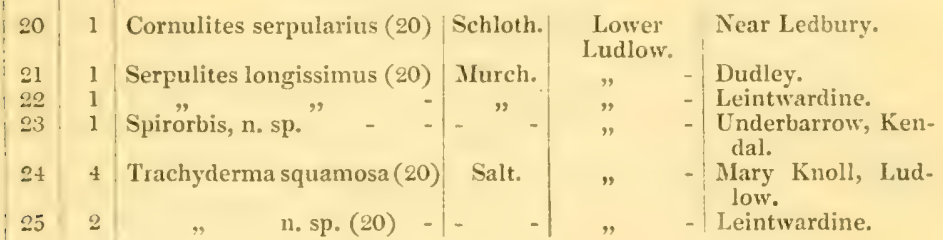

\section{CRUSTACEA.}

\begin{tabular}{|c|c|c|c|c|c|c|c|c|c|}
\hline 1 & 4 & \multicolumn{2}{|c|}{ Phacops caudatus } & - & & Briin. & Low & & Vinnal Hill, \\
\hline 2 & 1 & ", & " & - & & " & "ud! & & $\begin{array}{l}\text { Ludow. } \\
\text { N.E. of Leintwar- } \\
\text { dine. }\end{array}$ \\
\hline 3 & 1 & " & , & (20) & & , & $"$ & & $\begin{array}{l}\text { Bow Bridge, neat } \\
\text { Ludlow. }\end{array}$ \\
\hline 4 & 1 & $"$ & " & (20) & & ", & $"$ & - & Near Lcdbury. \\
\hline 5 & & " & Downingin ( & (20) & & Mur & $"$ & - & \\
\hline $6 a$ & 1 & $"$ & Downingle & - & & Mureno. & $"$ & & $\begin{array}{l}\text { Freshwater East. } \\
\text { Ledbury. }\end{array}$ \\
\hline 7 & 6 & $"$ & & (20) & & , & , & & Pont-ar-y-llechan. \\
\hline $\begin{array}{l}8 \\
9\end{array}$ & 1 & " & longicauda & latus $(2$ & & 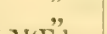 & ", & & Near Ledbury. \\
\hline 9 & 3 & ", & Stokesii & & & M.Edw. & , & & $\begin{array}{l}\text { Vinnal Hill, Luc } \\
\text { low. }\end{array}$ \\
\hline 16 & 2 & & Weaveri & - & & Salt. & " & & Tortworth. \\
\hline & 1 & Cheiru & us bimucror & natus & & Nurch. & " & & Le \\
\hline 13 & 2 & Acida & is Brightii & , & & NIurch. & $"$ & & Near The Wych, \\
\hline & 2 & " & coronatu & $1 \mathrm{~s}$ - & & Salt. & $"$ & & Vinnal Jill, Ludlo \\
\hline & 3 & $"$ & & 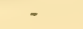 & & " & , & & , \\
\hline
\end{tabular}




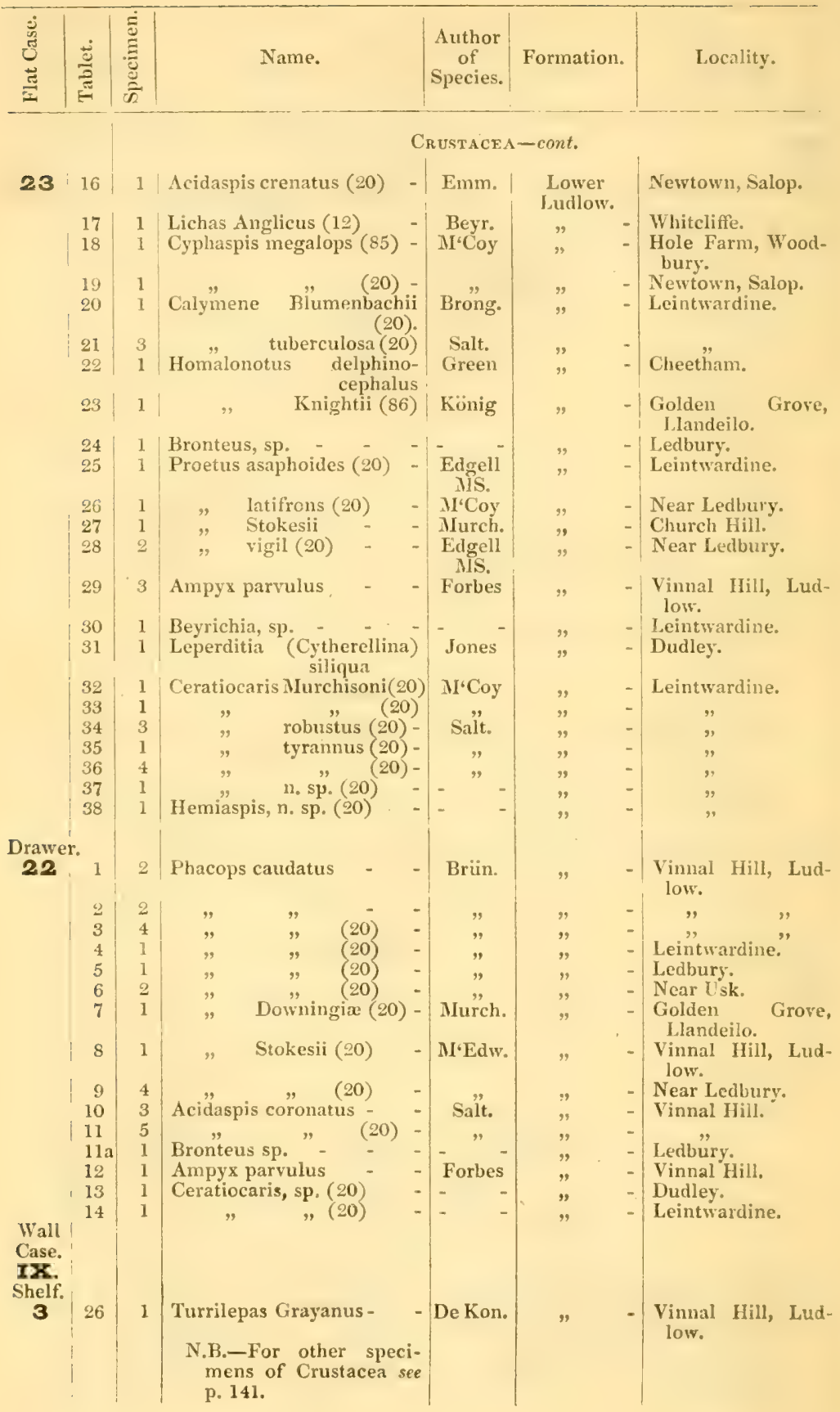




\begin{tabular}{|c|c|c|c|c|c|c|}
\hline 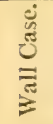 & है & 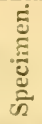 & Name. & $\begin{array}{c}\text { Author } \\
\text { of } \\
\text { Species. }\end{array}$ & Formation. & Locality. \\
\hline
\end{tabular}

IX.

Shelf.

\begin{tabular}{l|l}
3 & 27
\end{tabular}

\begin{tabular}{l|l|}
28 & 1 \\
29 & 3
\end{tabular}

30

Flat

Case.

\section{3}

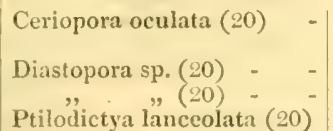

\section{BRACHIOPODA.}

Athyris compressi

Atryua reticularis $(20)$

Chonetes lepisma

$$
\begin{aligned}
& 2 \\
& 1 \\
& 1 \\
& 2 \\
& 3 \\
& 2 \\
& 2
\end{aligned}
$$

Meristella tumida

Spirifera crispa (20) -

" elevita

$$
" \quad, \quad( \pm 0)-
$$$$
\text { , }
$$$$
\text { exporrecti - }
$$

$$
\text { " plicatella (20) }
$$

Pentamerus rotundatus $(20)$

Rhynchonella navicula (20)

$$
\text { ," } \quad \text { Wucula }
$$

$$
\text { , , } \quad \text { (20) }
$$

\section{Leptæna sericea}

", transversalis (20)

Orthis biloba

$$
\text { , crassa }
$$

$$
\text { " elegantula }
$$

$$
\text { hybrida - }
$$

Strophomena antiquata

$$
\text { " fuglypha }
$$

rhomboidalis

$$
\text { (lavigata, Eth.) (20). }
$$

$$
\text { , minima }
$$

Cr" $(20)$

Discina rugata $(20)$ -

Lingula lata

Sby.

Linn.

Dalm.

His.

Daln.

Wäh!.

Lin!

Sby.

"$$
\text { , }
$$

$$
\text { " }
$$

Wahl.

Linn.

Lindst.

Dalm.

Sby.

His.

Sby.

Wilck.

Sby.

$$
\begin{aligned}
& \text { Lewisii (20) } \\
& \text { minima }(20) \\
& \text { striata - } \\
& \text { Symondsii (20) }
\end{aligned}
$$

Obolus Davidsoni (20)

\section{POLYZOA.}

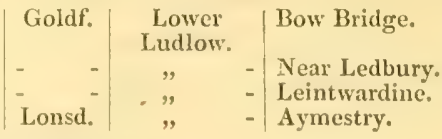

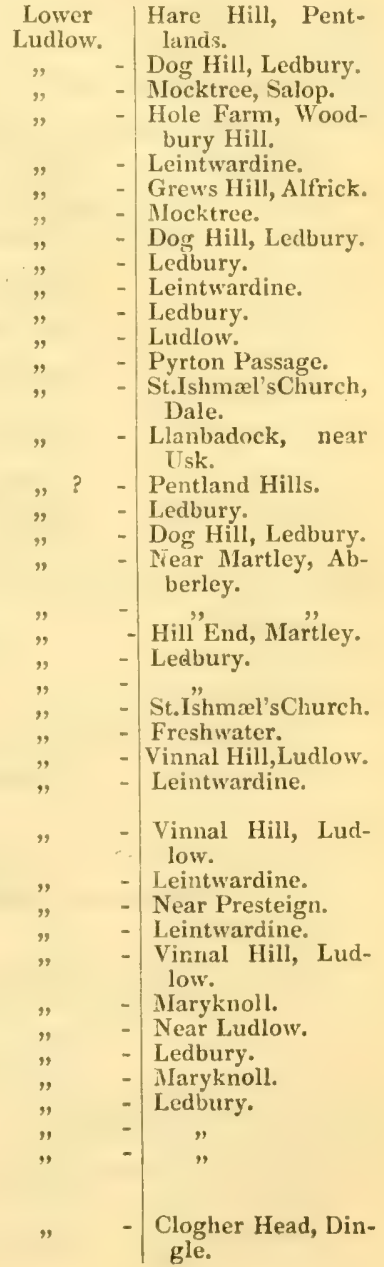




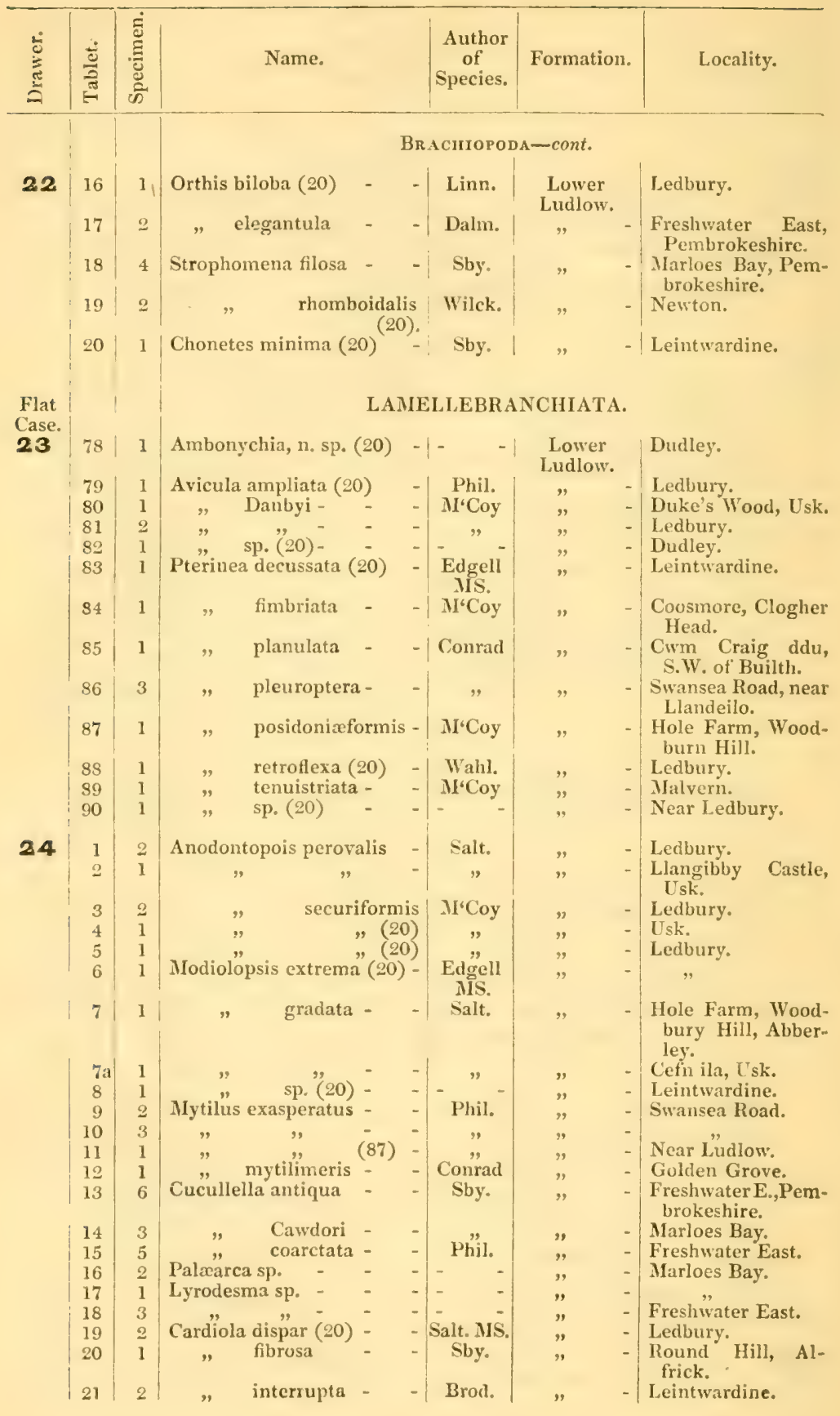




\begin{tabular}{|c|c|c|c|c|c|c|}
\hline 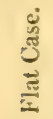 & 苑 & 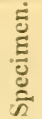 & Name. & $\begin{array}{l}\text { Author } \\
\text { of } \\
\text { Species. }\end{array}$ & Formation. & Locality. \\
\hline
\end{tabular}

\section{LAMELLIBRANCHIATA-cont.}

24

Drawer.

22

Flat

Case.

24

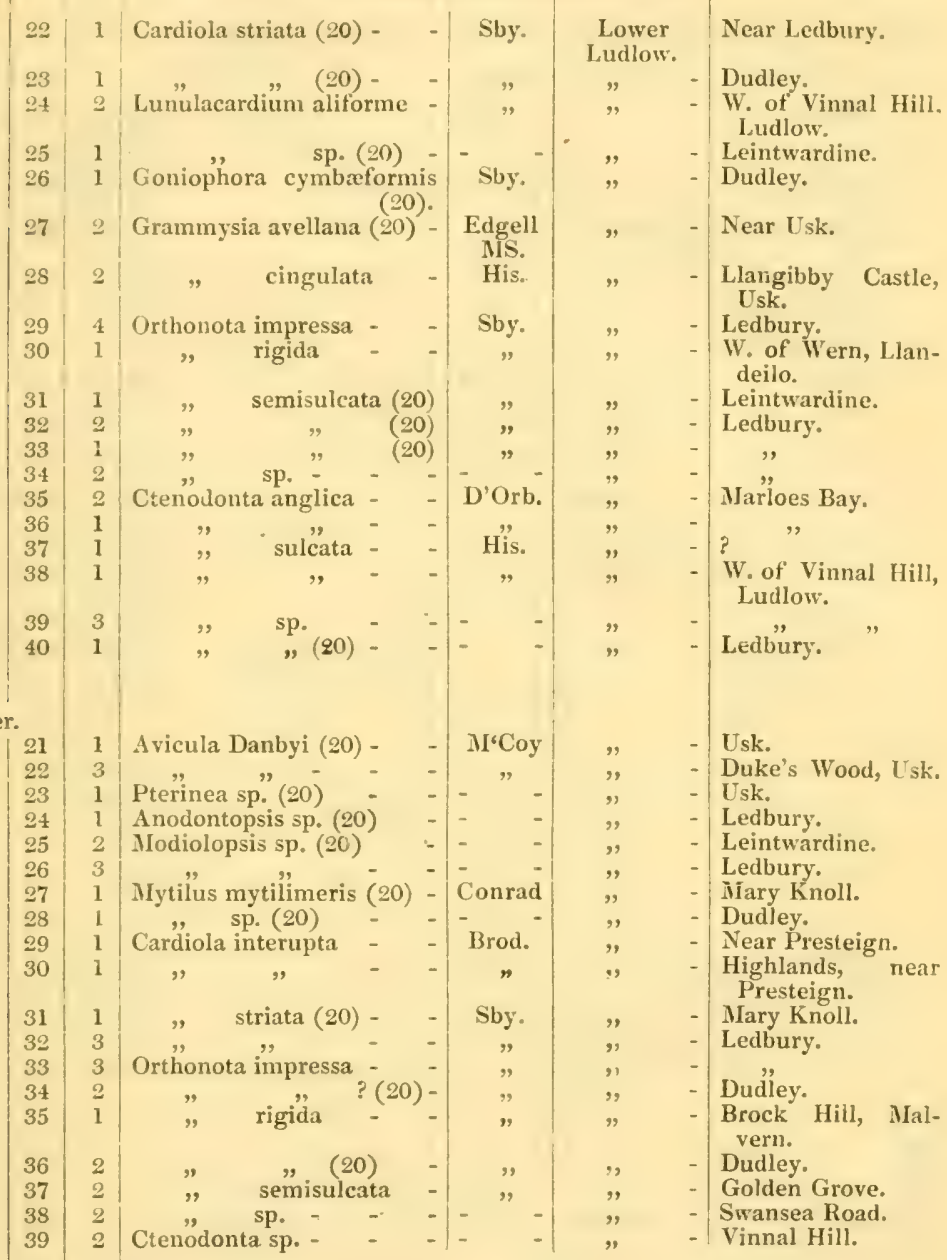

41 42 42
43
44

\section{GASTEROPODA. \\ GASTEROPODA.}

Murchisonia articulata

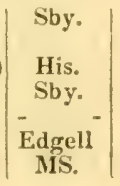

Lower Ludlow. Underoarrow, Kendal. Near Ledbury.

Llangibby Castle,

W. of Wern, Llandeilo.

?

Marloes Bay.

W. of Vinnal Hill, Ludlow.

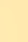




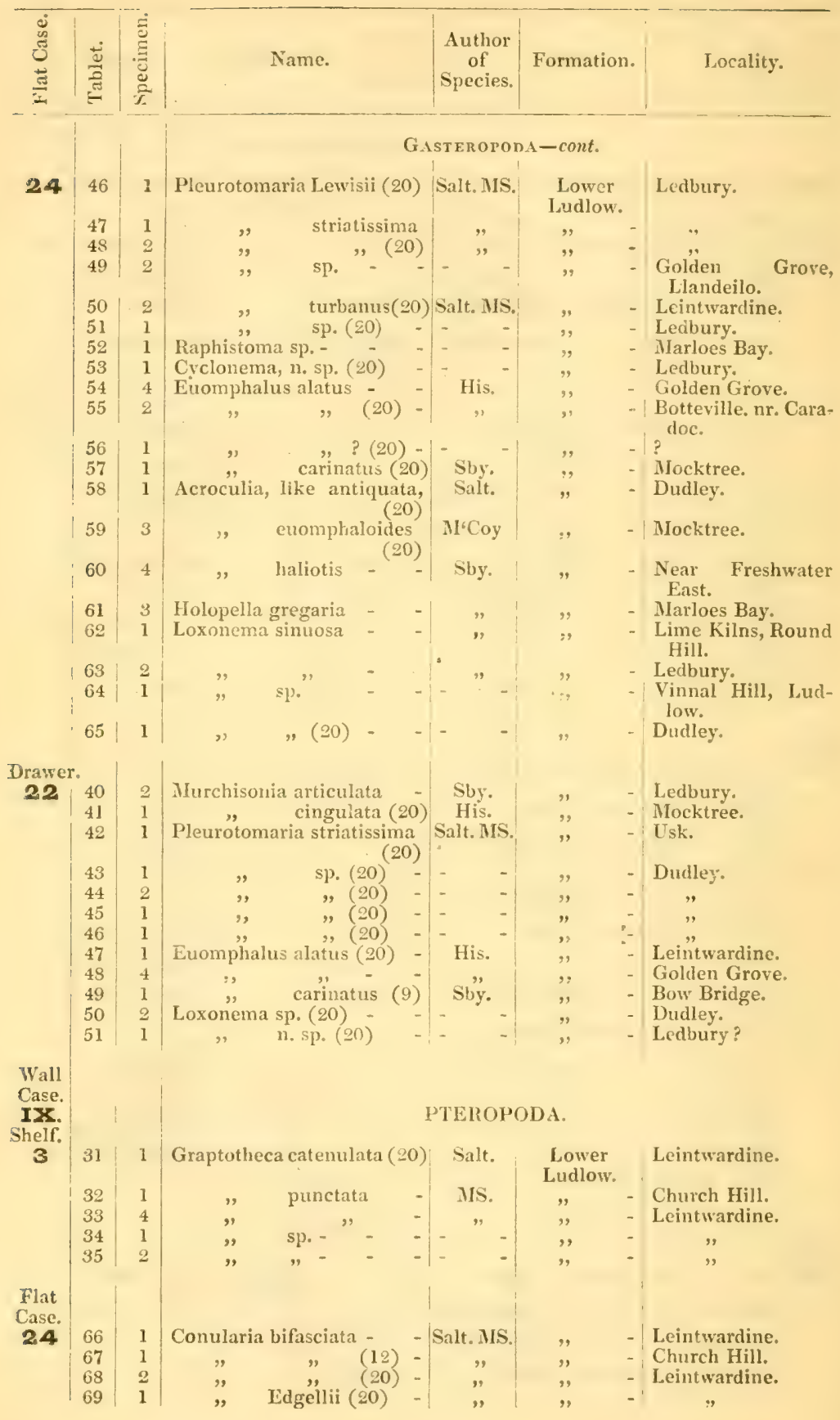




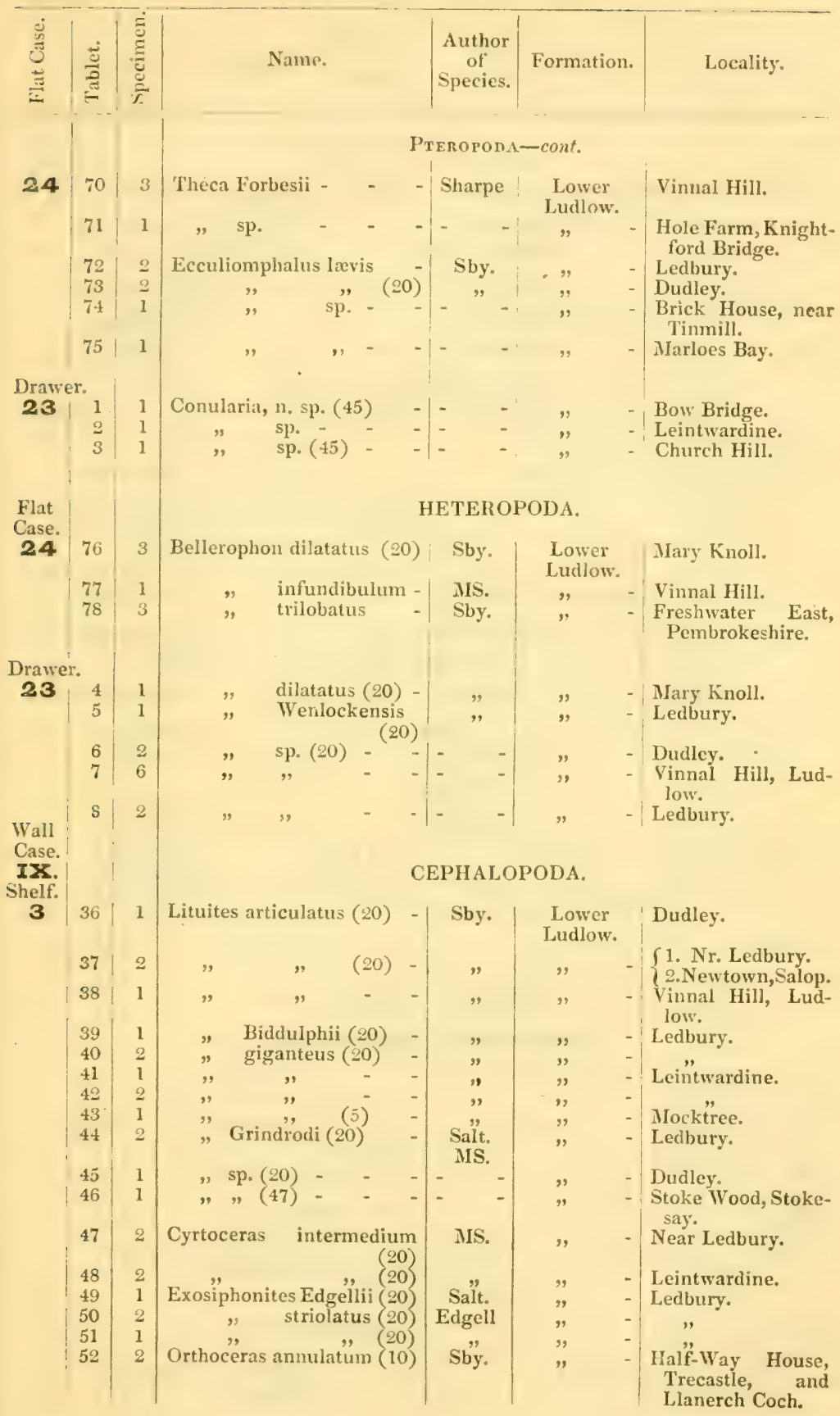




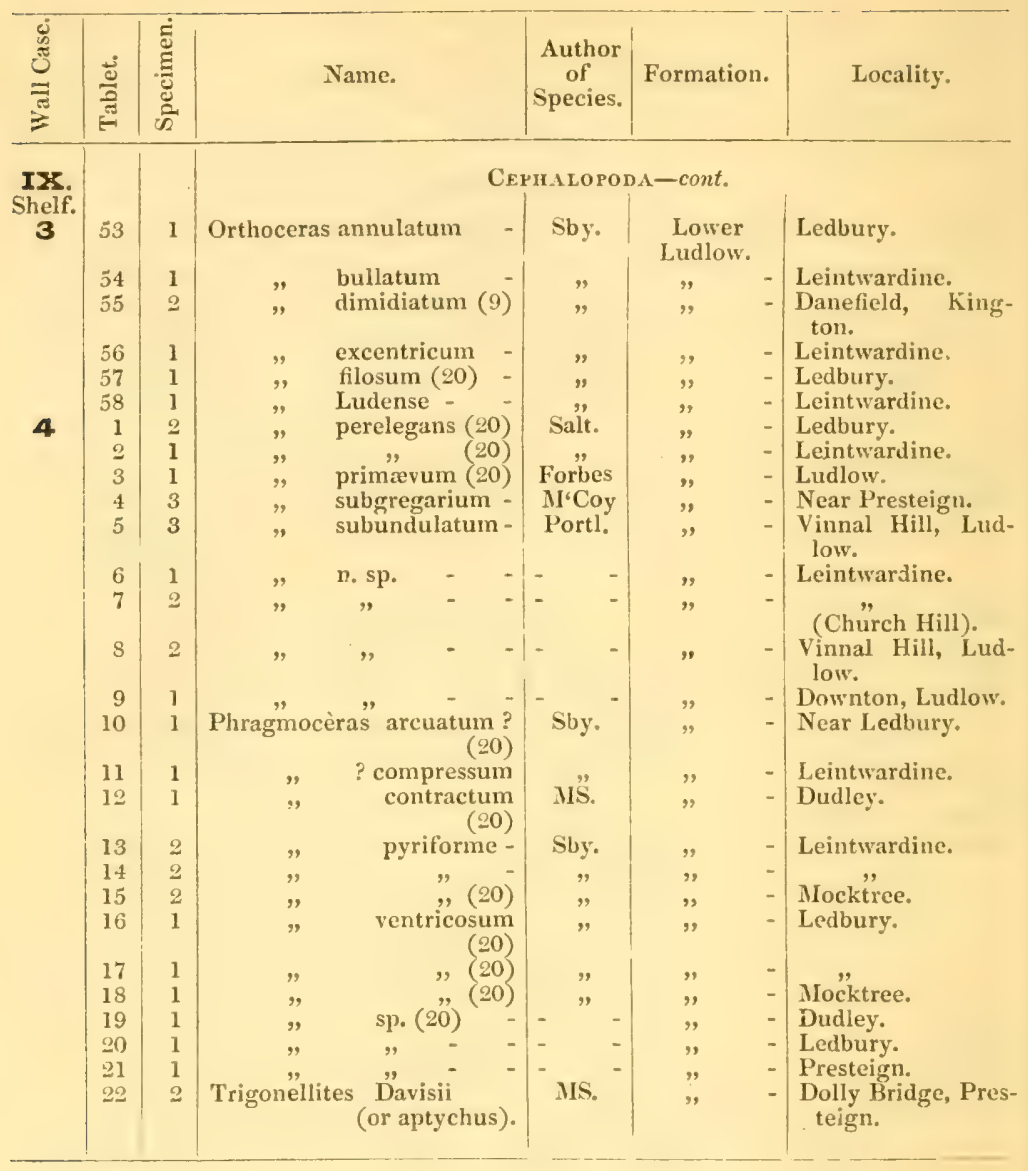

UPPER SILURIAN-cont,

AXMRSTRY IMMESTONE.

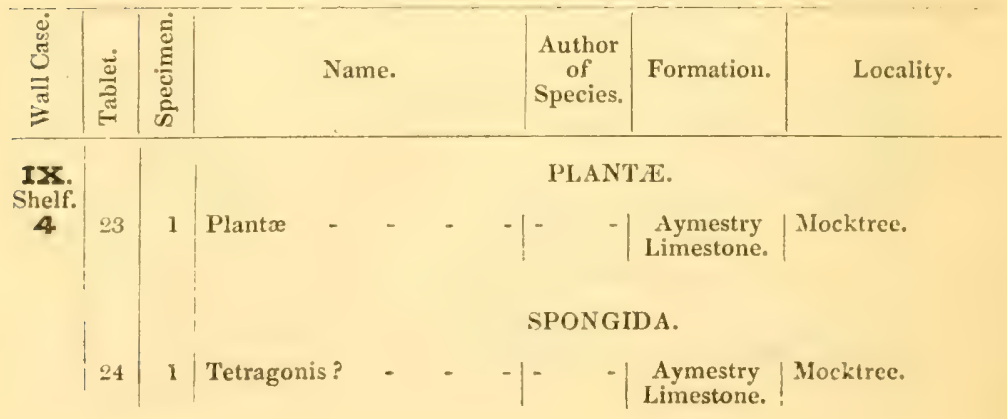




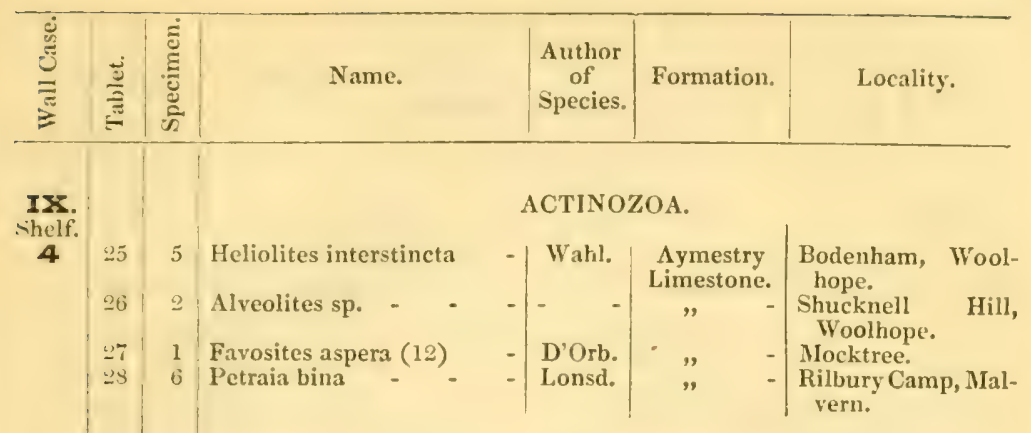

Flat

Case.

25

Flat

Case.

25

Case

IX.

4

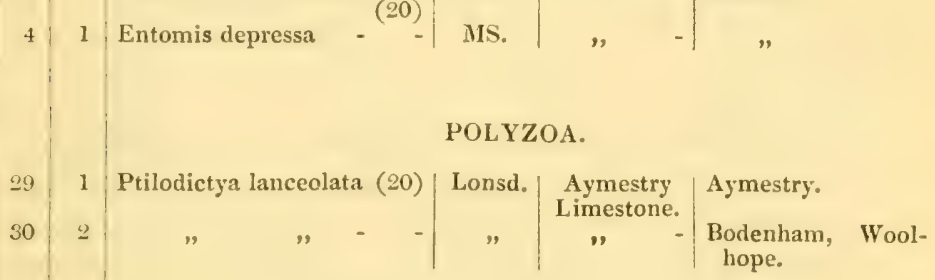

29
30

Ptilodictya lanceolata (20)

POL YZOA.

\section{CRUSTACEA.}

Briin. Aymestry Barkbury Camp. Limestone.

\section{Bodenham.}

Mocktree.

\section{BRACHIOPODA.}

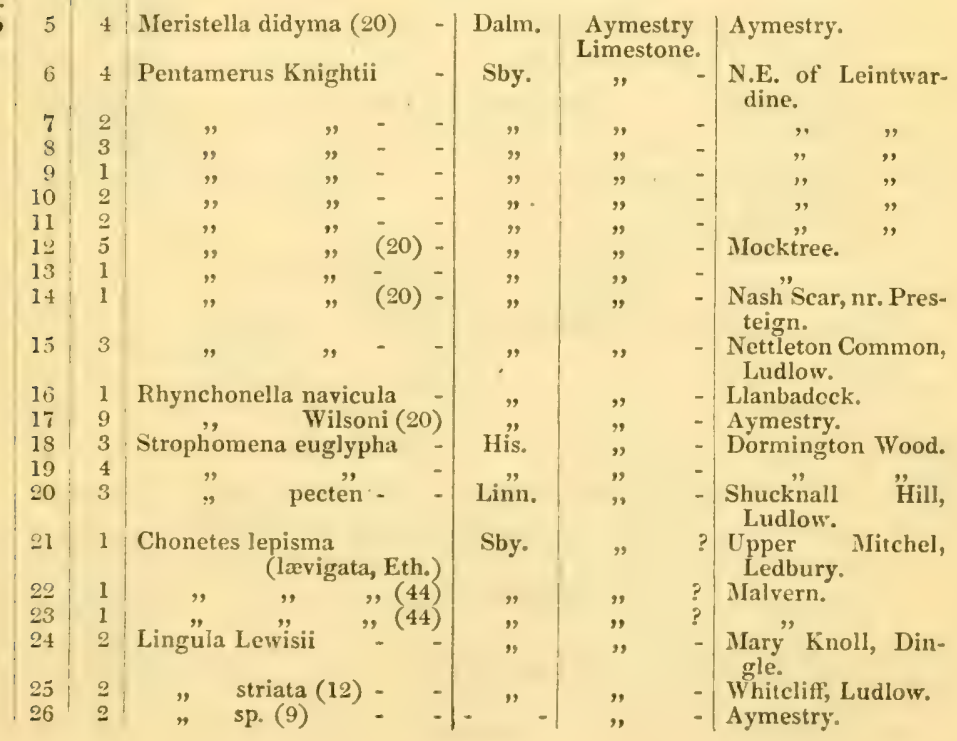




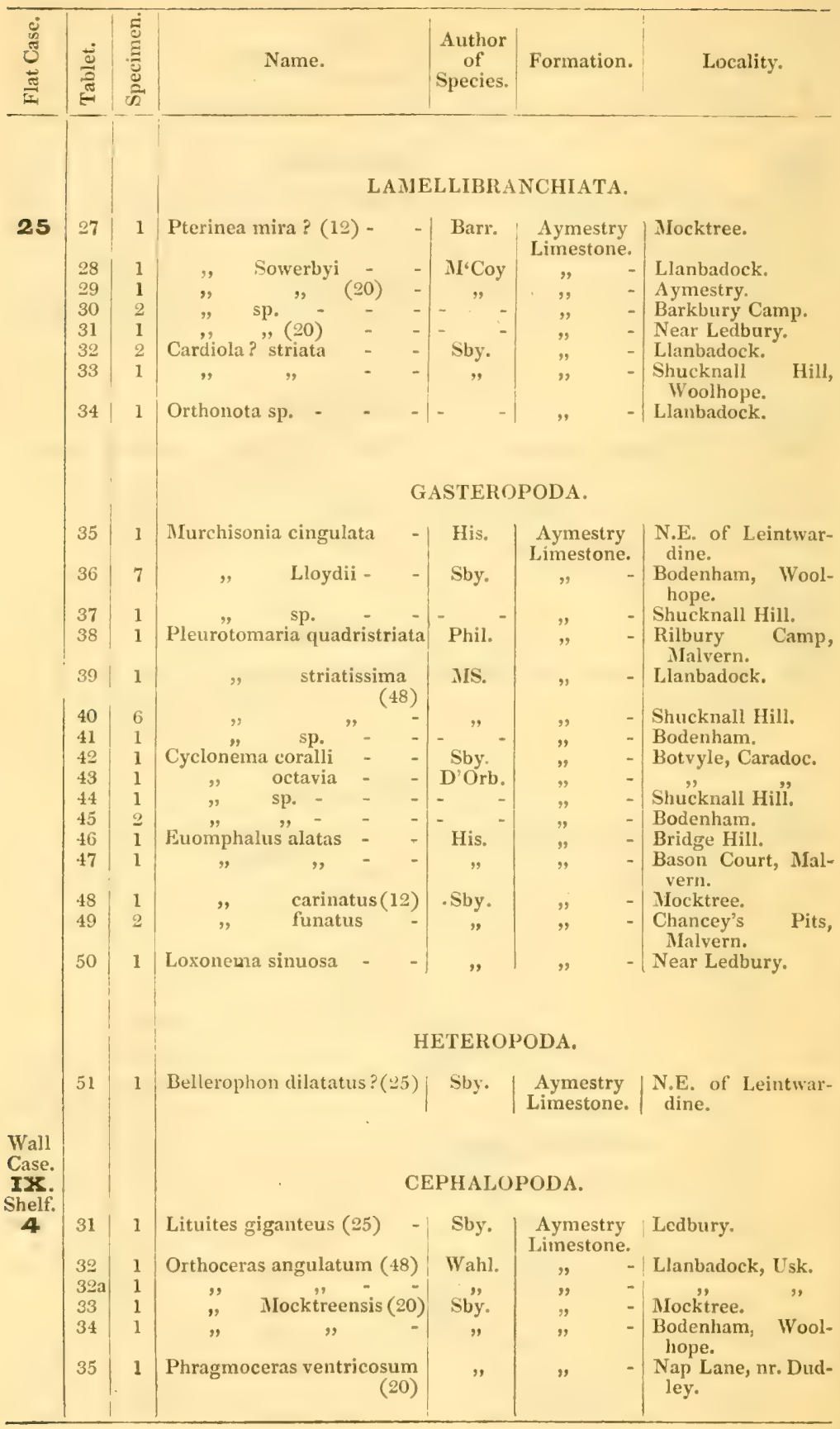


TPPER SILURIAN-cont.

UPPER XUDXOW.

\begin{tabular}{|c|c|c|c|c|c|c|}
\hline 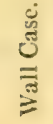 & 芯 & 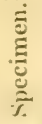 & Name. & $\begin{array}{l}\text { Author } \\
\text { of } \\
\text { Species. }\end{array}$ & Formation. & Locality. \\
\hline
\end{tabular}

VARIOUS LARGE SPFCIMENS.

IX.

Shelf.

1

\begin{tabular}{l|l|l} 
Theca Forbesii - - & 9 \\
(Orthoceras bullatum -
\end{tabular}

\begin{tabular}{|l|l|l|l|} 
& 10 & 1 & p
\end{tabular}

Favosites fibrosa

111 Orthis lunata - - $\quad-$ Sby.

Rhynchonella nucula

121 Orthoceras bullatum -

131 Avcullella, Holopella, \&c. -

14 I Heterocrinus? (12)

151 Modiola and Tentaculites - -

\begin{tabular}{l|l|l}
16 & 1 & Rhynchonella navicula \\
\hline
\end{tabular}

171 Modiolopsis

\begin{tabular}{l|l|l}
18 & 1 & Serpulites longissimus
\end{tabular}

19

20

1 Orthoceras lbex

Covered with

Monticulipora papillata.

1 Monticulipora -

\author{
Sharp |\} Upper ?| Kington, Hereford. \\ Salt f Ludlow. \\ siby. $\}=-\left\{\begin{array}{l}\text { Batshot, } \\ \text { low. }\end{array}\right.$ \\ Sby. \{ Frith Quarry, \\ Dahn. $\},-\{$ Stapleton, Pres- \\ Sby. $\},\{$ teign. \\ f Trichneg, Llan-

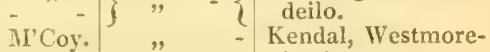 \\ land. \\ - Whitcliff, Ludlow. \\ - 2 m. S. of Llan- \\ deilo. \\ - Pyrton Passage. \\ - Storm Hill Lodge, \\ Llandeilo. \\ - Presteign. \\ - Brigsteer. \\ - Croagh Mulim \\ Doonquin, Dingle.
}

\section{PLANTA.}

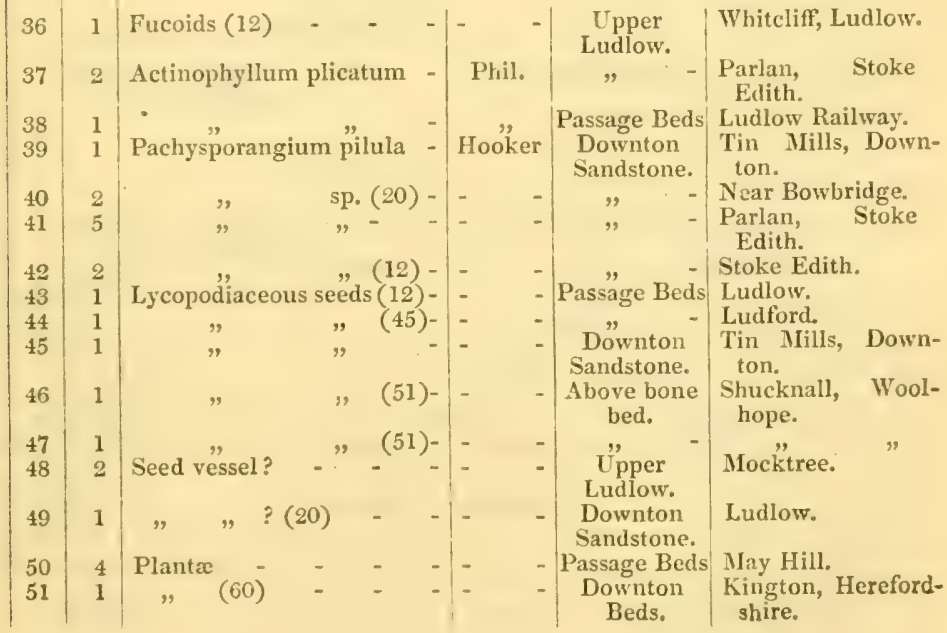




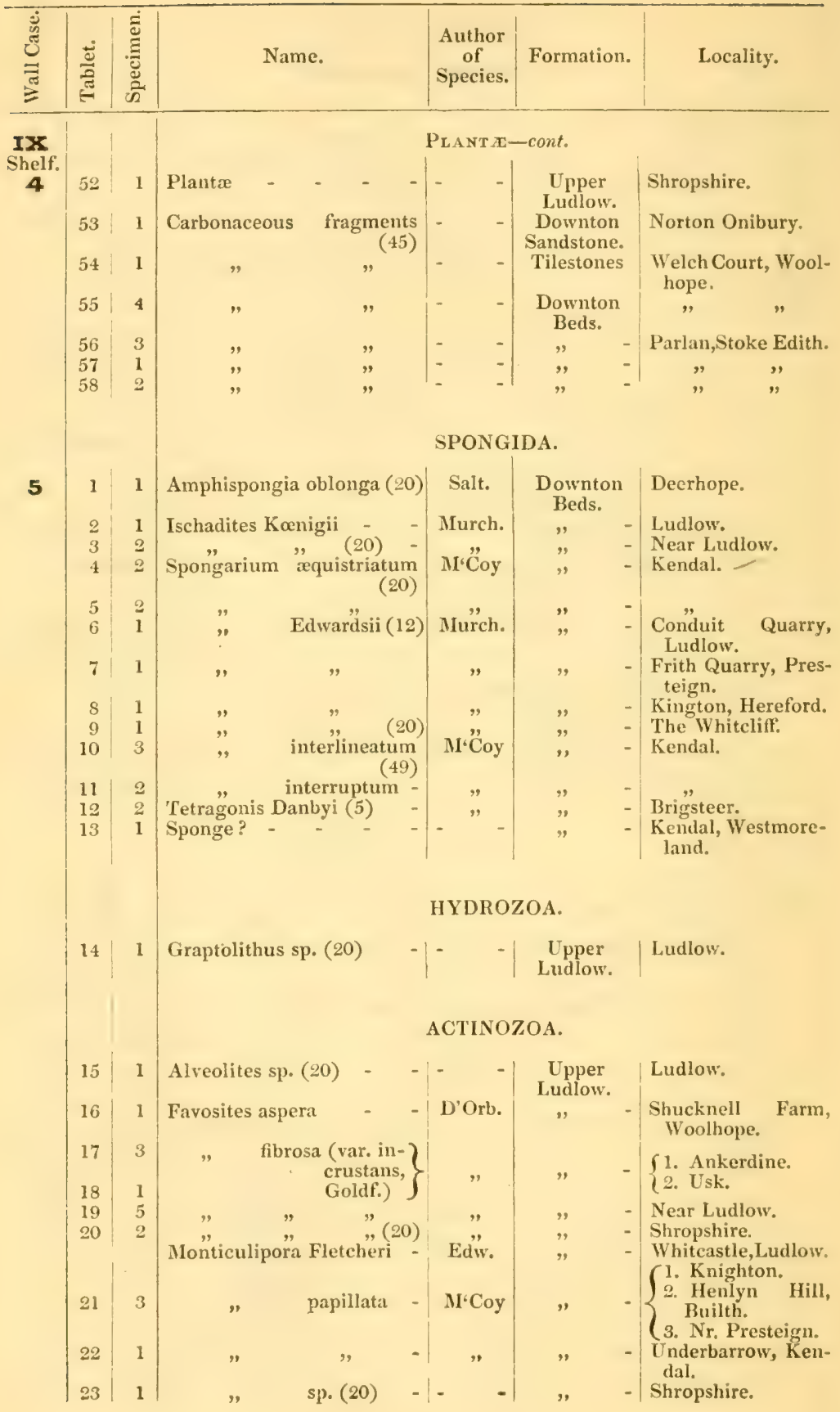




\begin{tabular}{|c|c|c|c|c|c|c|}
\hline$\sum_{\bar{J}}$ & 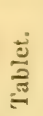 & 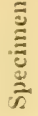 & Name. & $\begin{array}{l}\text { Author } \\
\text { of } \\
\text { Species. }\end{array}$ & Formation. & Locality. \\
\hline
\end{tabular}

Ix.

Shelf:

Actinozo.1-cont.

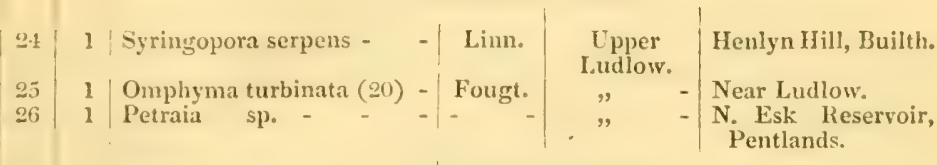

\section{ECHINODERMATA.}

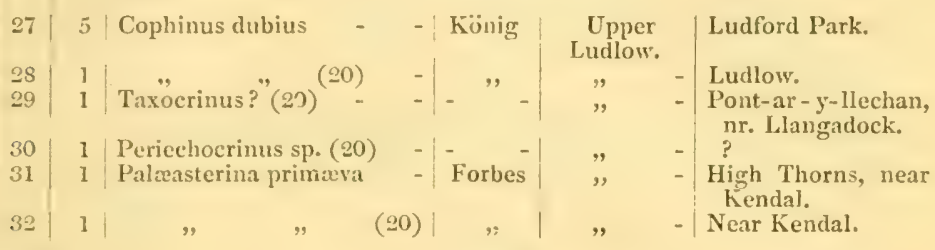

ANNELDA.

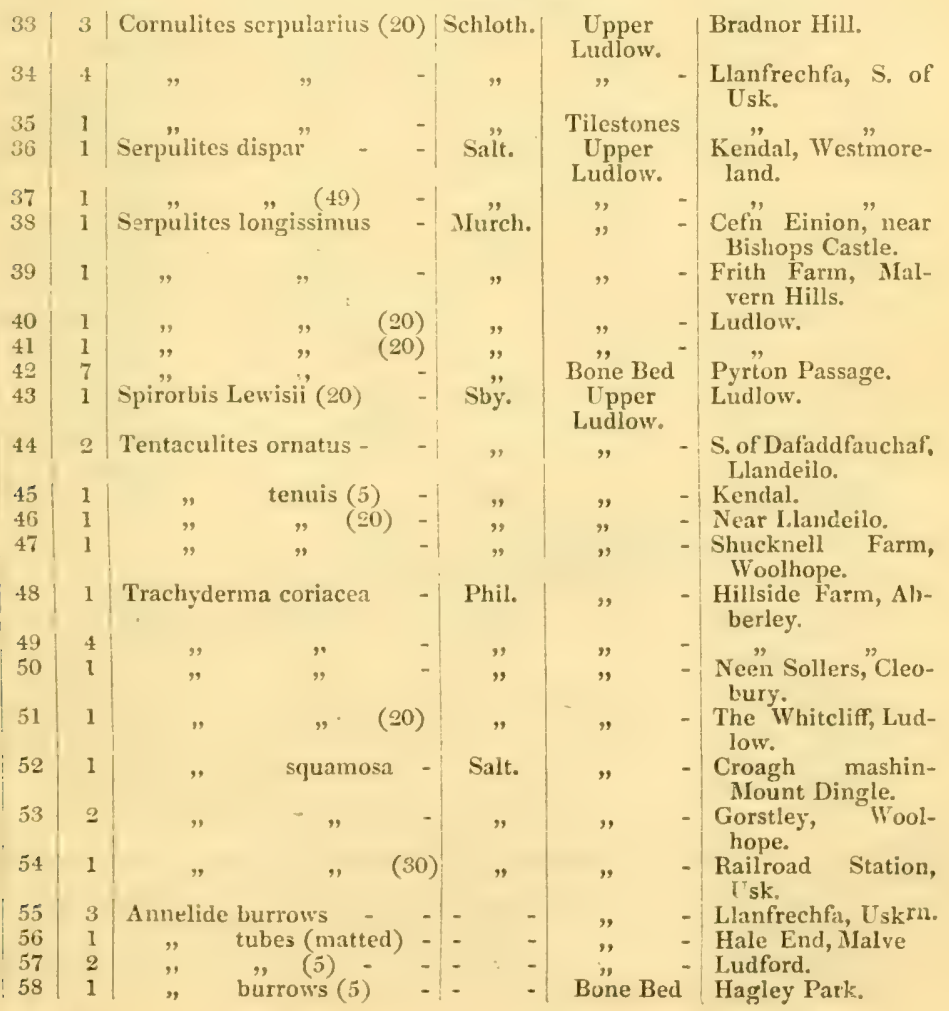




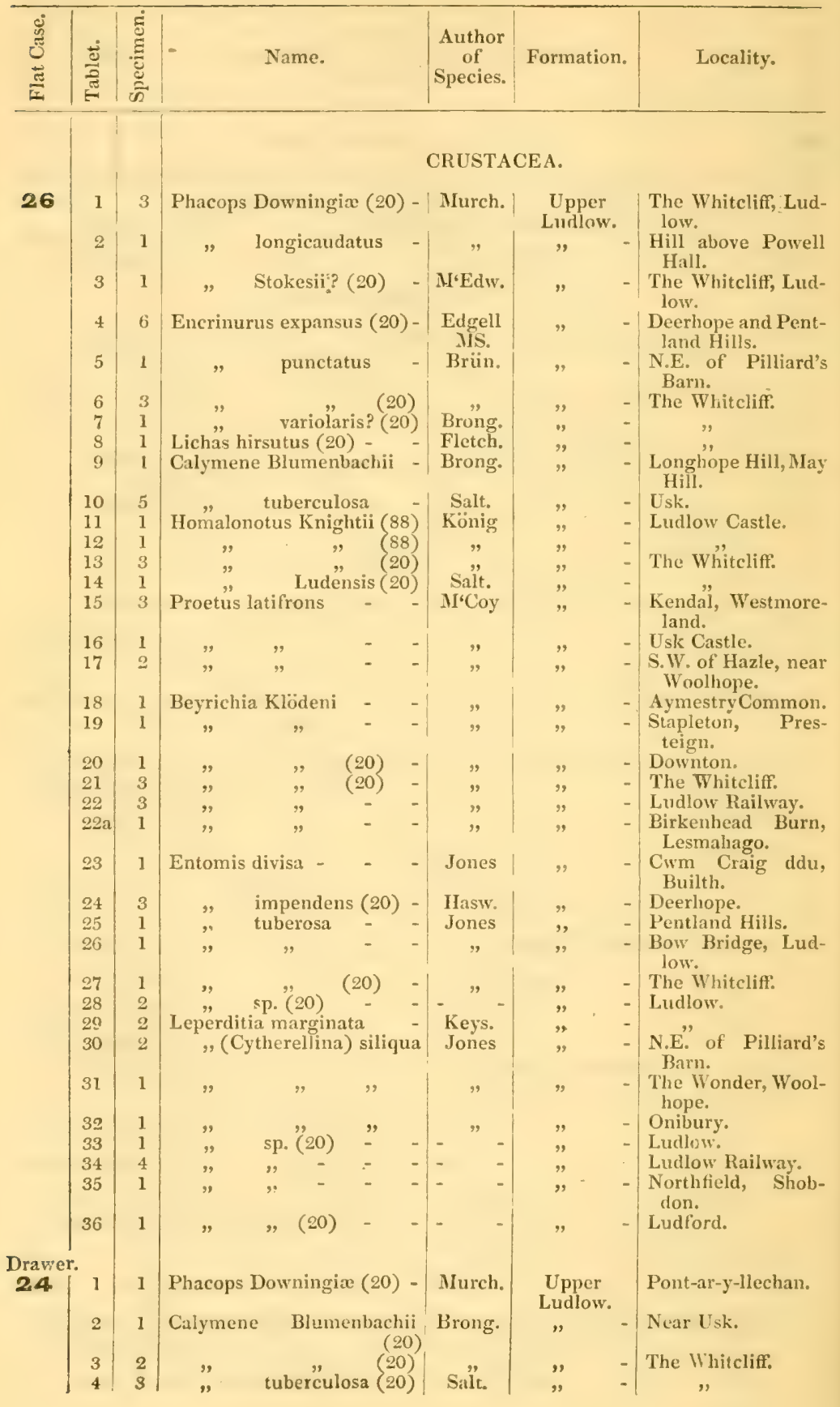




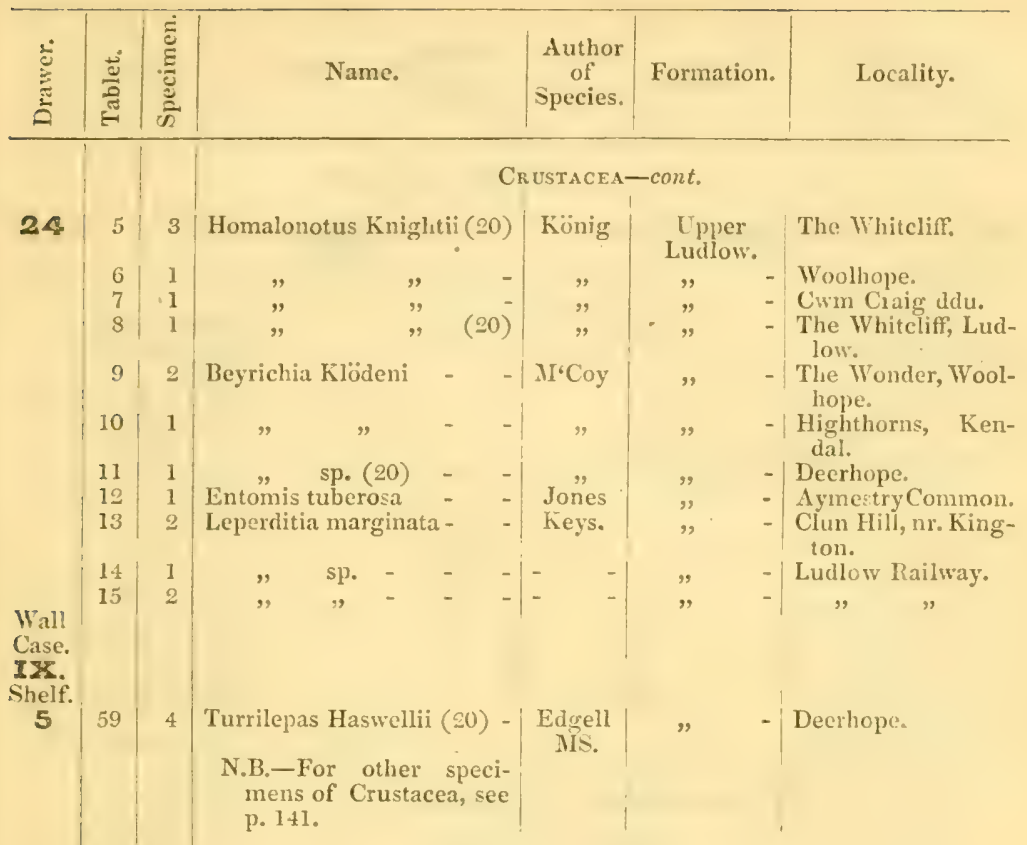

\section{POLYZOA.}

6

Flat Case.

\section{BRACHIOPODA.}

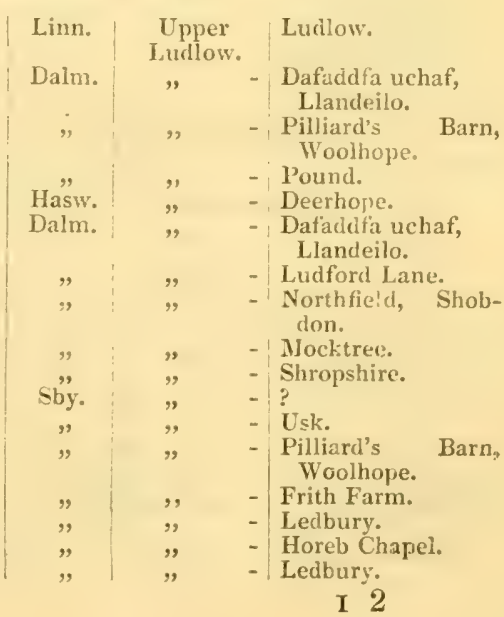




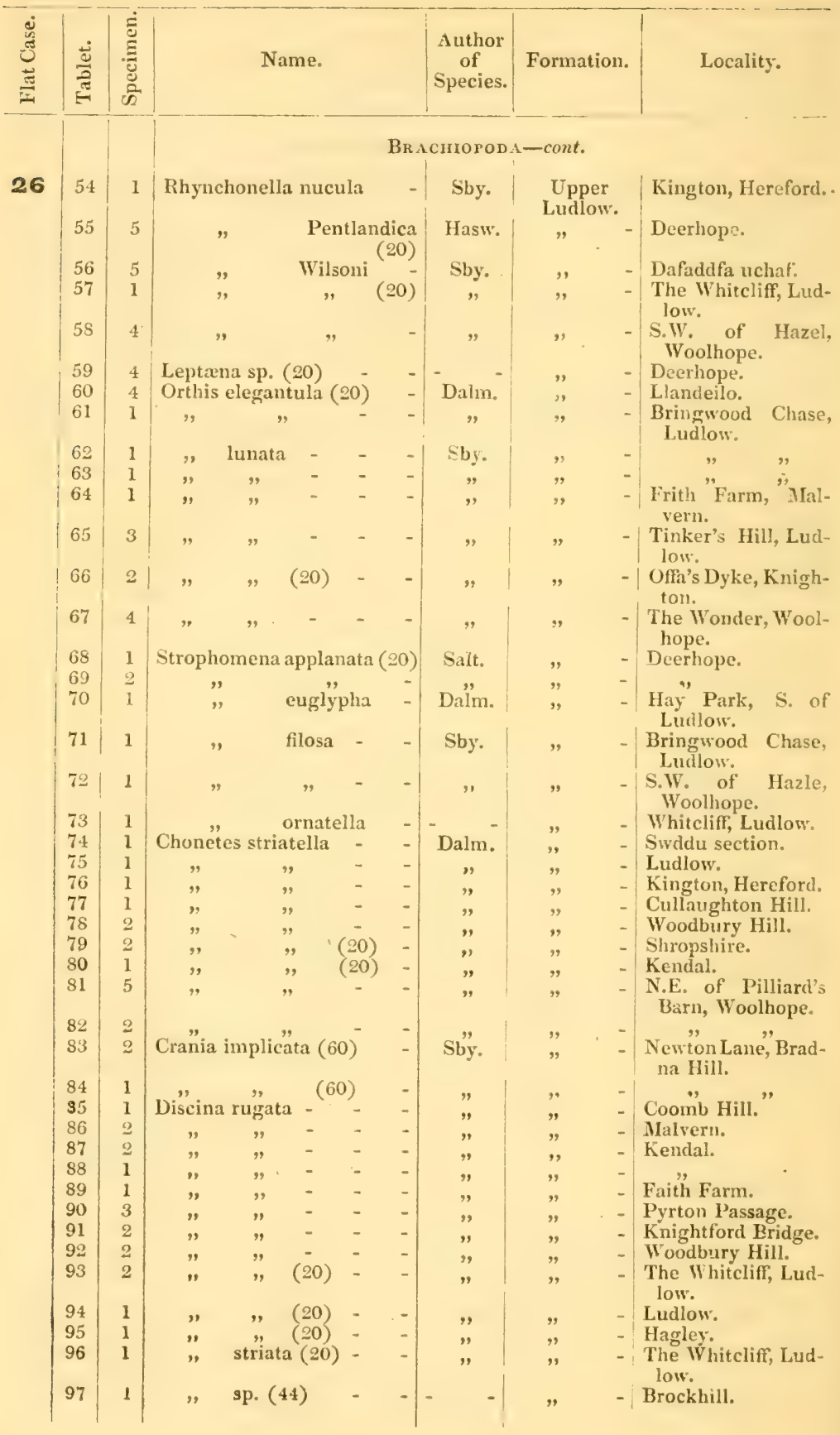




\begin{tabular}{|c|c|c|c|c|c|c|}
\hline 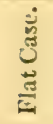 & & 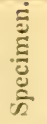 & Name. & $\begin{array}{l}\text { Author } \\
\text { of } \\
\text { Species. }\end{array}$ & Formation. & Locality. \\
\hline
\end{tabular}

\section{BrACHIOPODA-cont.}

27

\begin{tabular}{|c|c|c|c|c|c|c|c|c|c|}
\hline 1 & 2 & Lingula cornea & & - & - & Sby. & Upper & & Lesmahago. \\
\hline 2 & 2 & " & - & - & - & " & ,y & - & Kendal. \\
\hline & 3 & ", & (20) & - & - & , & $"$ & 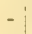 & Downton. \\
\hline & 3 & " & (12) & - & - & ", & " & - & Ludlow Railsvay. \\
\hline & 3 & ," & - & - & - & " & " & & $\begin{array}{l}\text { Kington, Hererora } \\
\text { shire. }\end{array}$ \\
\hline
\end{tabular}

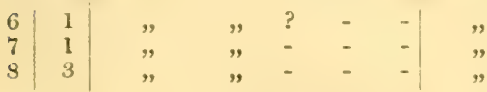

Onibury.

- Dean's Corner, E. of Wenlock.

- Ludford.

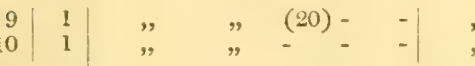

River Teme, Luil. low.

Brockhill.

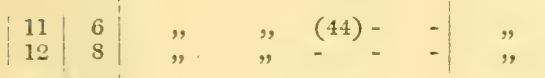

Maes - y - gwastad, Kington.

! Clun Hill, near Kington.

Birkenhead Burn, Lesmahago.

- Kendal, Westmoreland.

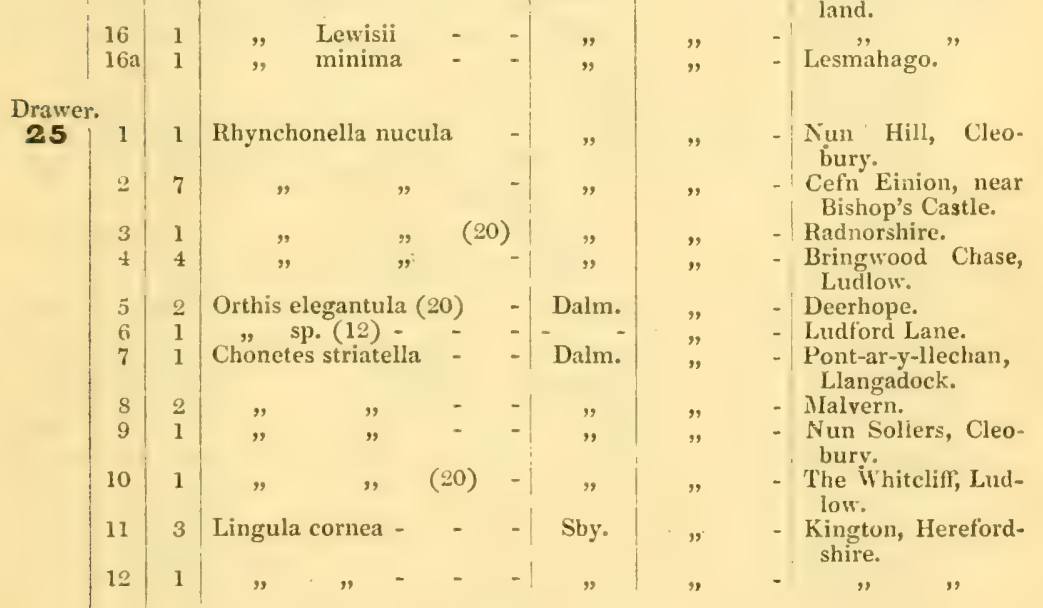

Flat

LANELLIBRANCHIATA.

27

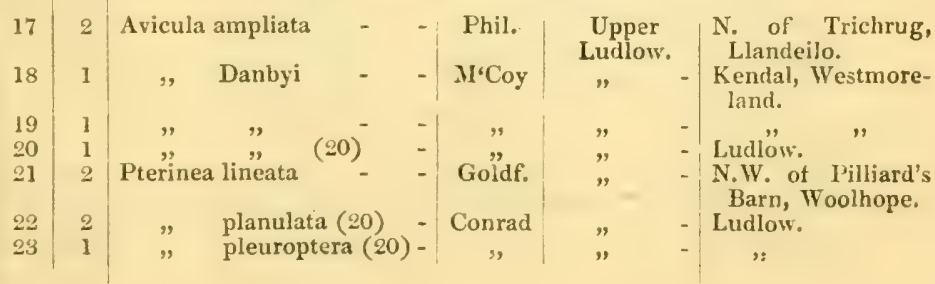




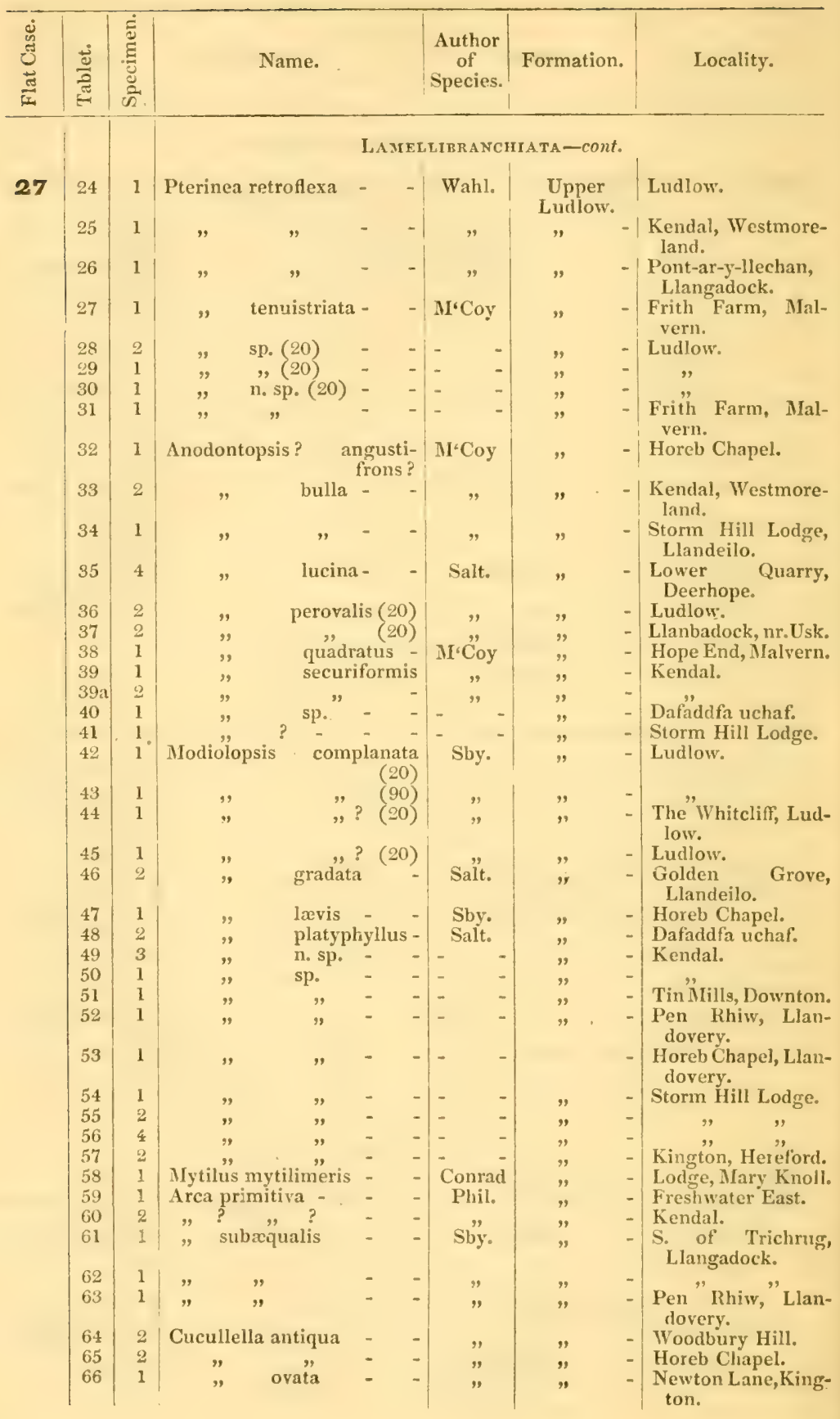




\begin{tabular}{|c|c|c|c|c|c|c|}
\hline$\underset{\tilde{\Xi}}{\stackrel{0}{0}}$ & $\frac{\frac{3}{3}}{3}$ & 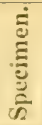 & Name. & $\begin{array}{c}\text { Author } \\
\text { of } \\
\text { Species. }\end{array}$ & Formation. & Locality. \\
\hline
\end{tabular}

LAMEITIBRANCHIATA-cont.

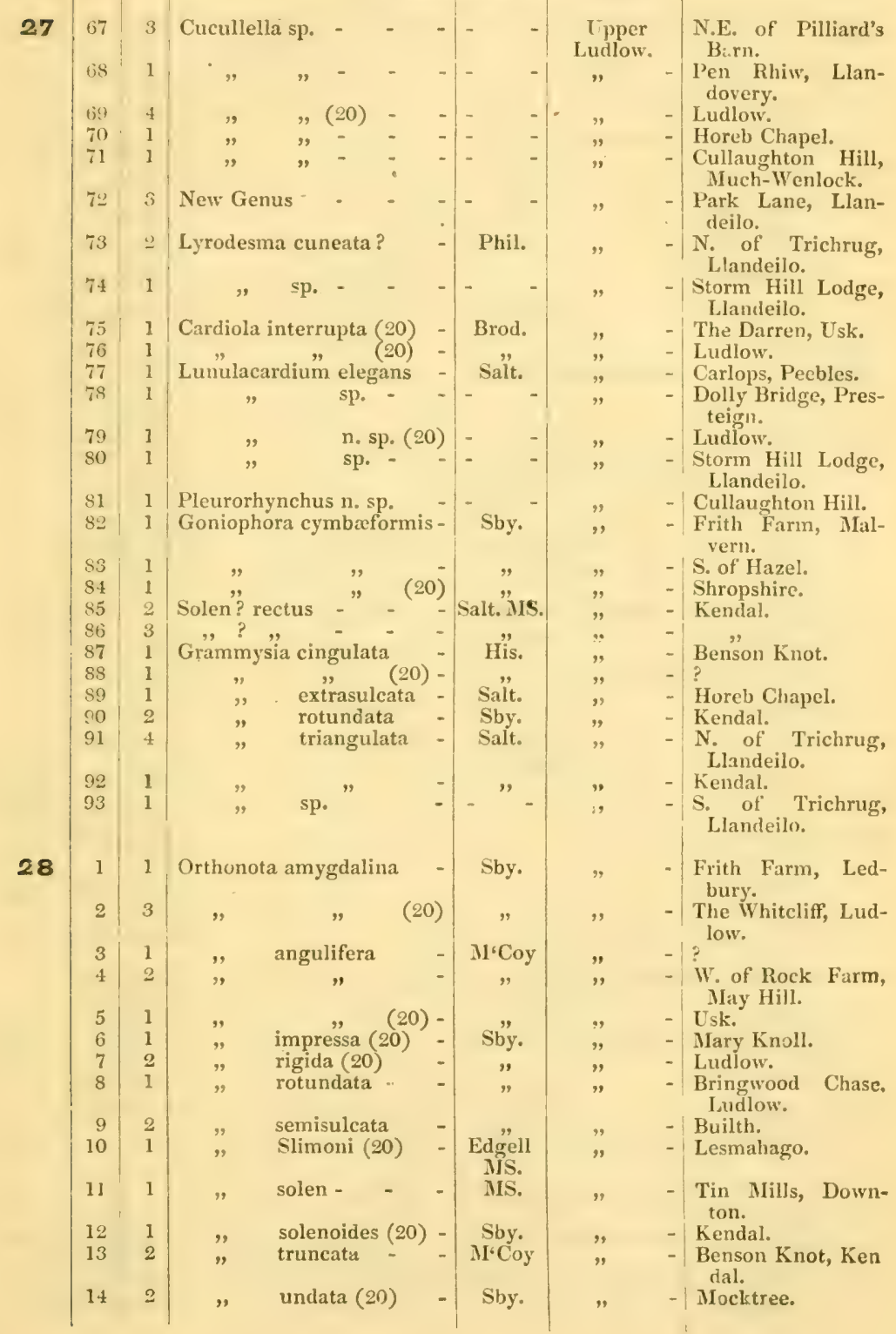




\begin{tabular}{|c|c|c|c|c|c|c|}
\hline 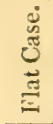 & 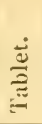 & 苛 & Name. & $\begin{array}{l}\text { Author } \\
\text { of } \\
\text { Species. }\end{array}$ & Formation. & Locality. \\
\hline
\end{tabular}

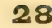

28

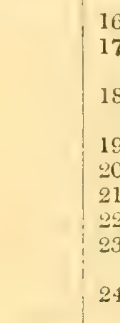

Drawer.

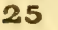

LAMELLIBR.IXCHITA-cont.

$-\frac{1}{2}$

$$
\begin{array}{c|l}
\begin{array}{c}
\text { Ipper } \\
\text { Ludlow. }
\end{array} & \begin{array}{l}
\text { Lower } \\
\text { Deerhope. } \\
,,
\end{array} \\
\text { Ludlow. }
\end{array}
$$

- Deerhope.

- Ludlow.

Horeb Chapel, Llandovery.

Carlops, Pcebles.

Kendal.

Avicula Danbyi (20) - $\quad$ - $\mathbb{M}^{6} \mathrm{Coy}$

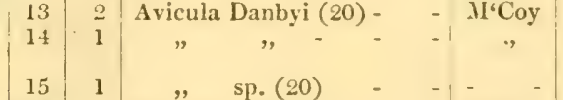

Meas Clettur, near Builth.

- The Whitclift.

- S. of Hazel, Woolhope.

- Ludlow.

- Kendal.

- The Whitcliff.

Ludlow.

- Kendal.

Neä Llandovery.

Storm Hill Lodge.

- Kendal.

- Helme Fort.

Golden Grove.

- S. of Trichrug.

- Kendal.

- Blaeberry Burr, Logan Water, Lesmahago.

- Kington, Herefordshire.

- Storm Hill Lodge.

- Isudlow.

Storm Hill Lodge.

Modiola, sp. (20)

| " " (20)

| Cucullella, sp. -

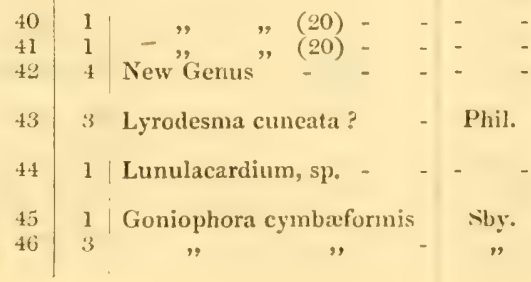

- Lesmahago.

Kington.

- Ludlow.

- Hillside Farm, Woodbury Hill, Abberley.

- Ludlow.

Park Lane, Llandcilo.

- N. of Trichrug, I.landeilo.

Coombe Wood, Presteign.

Malvern.

Llanfrechfa, Usk. 


\begin{tabular}{|c|c|c|c|c|c|c|}
\hline 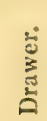 & 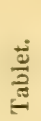 & 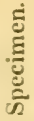 & Name. & $\begin{array}{c}\text { Author } \\
\text { of } \\
\text { Species. }\end{array}$ & Formation. & Locality. \\
\hline
\end{tabular}

\section{LAMELLIBRANCHIAT-cont.}

25

\section{6}

Flat

Case.

28

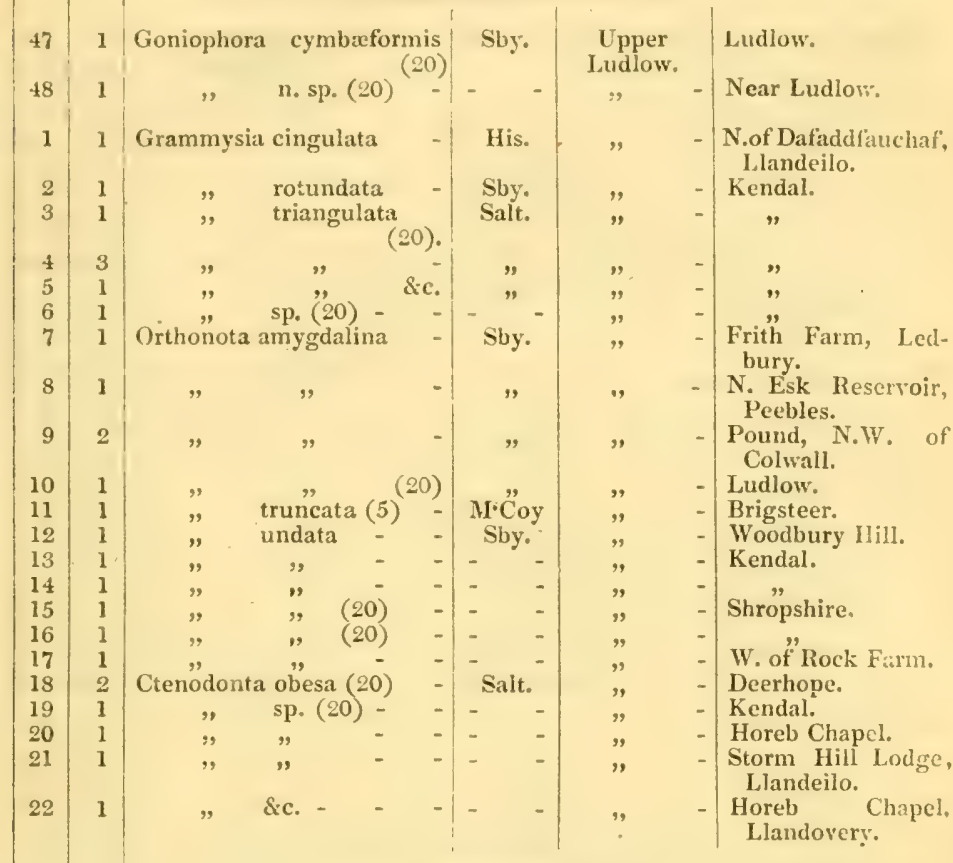

\section{GASTEROPODA.}

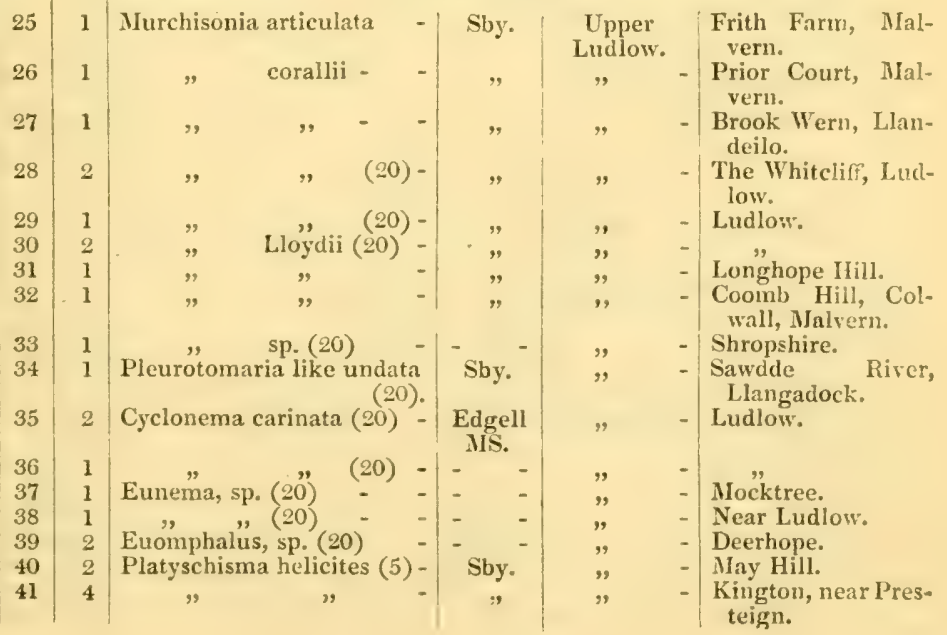




\begin{tabular}{|c|c|c|c|c|c|c|}
\hline 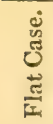 & $\frac{\vec{d}}{d \overrightarrow{0}}$ & $\begin{array}{l}\text { ङूँ } \\
\text { हूँ } \\
\text { कू } \\
\text { के }\end{array}$ & Name. & $\begin{array}{l}\text { Author } \\
\text { of } \\
\text { Species. }\end{array}$ & Formation. & Locality. \\
\hline
\end{tabular}

28

\begin{tabular}{|l|l}
4 \\
4 \\
4 \\
4 \\
47 \\
4 \\
4 \\
5 \\
5 \\
5 \\
55 \\
5 \\
55 \\
5 \\
57 \\
58 \\
59 \\
60 \\
6 \\
69 \\
63 \\
64 \\
65 \\
66 \\
67 \\
68 \\
69 \\
70 \\
7 \\
7 \\
7 \\
5
\end{tabular}

Drawer.

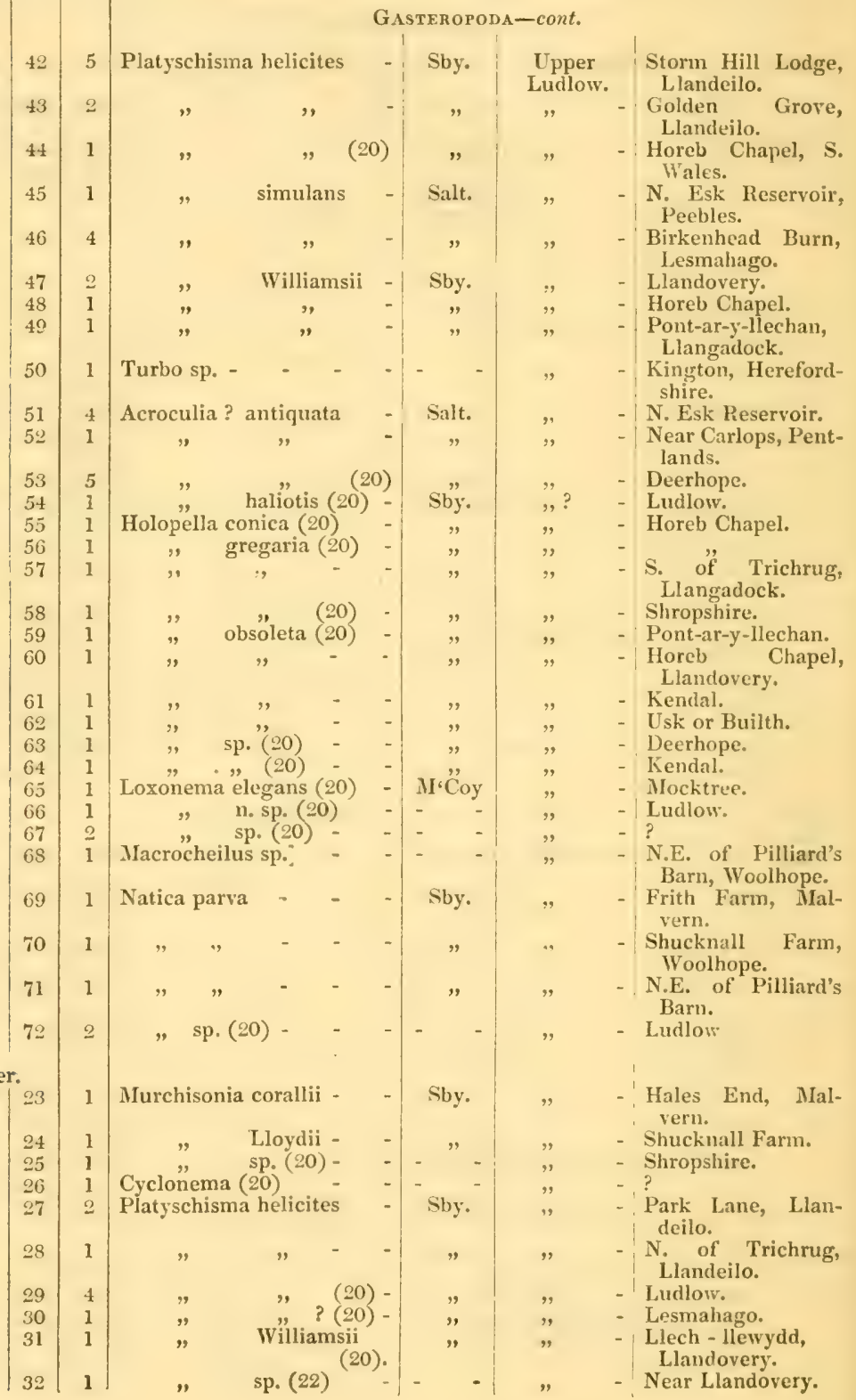




\begin{tabular}{|c|c|c|c|c|c|c|}
\hline 苛 & 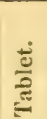 & 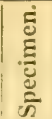 & Name. & $\begin{array}{c}\text { Author } \\
\text { of } \\
\text { Species. }\end{array}$ & Formation. & Locality. \\
\hline
\end{tabular}

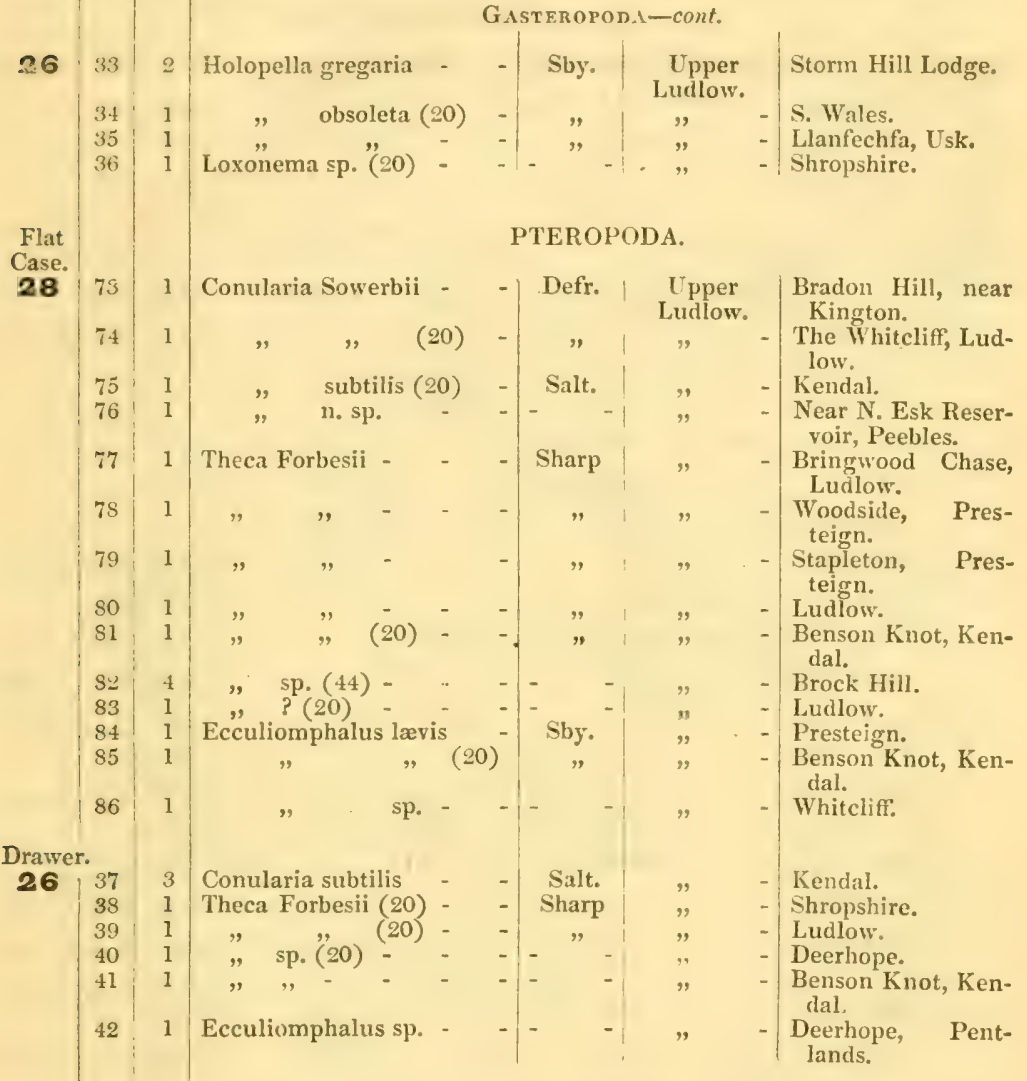

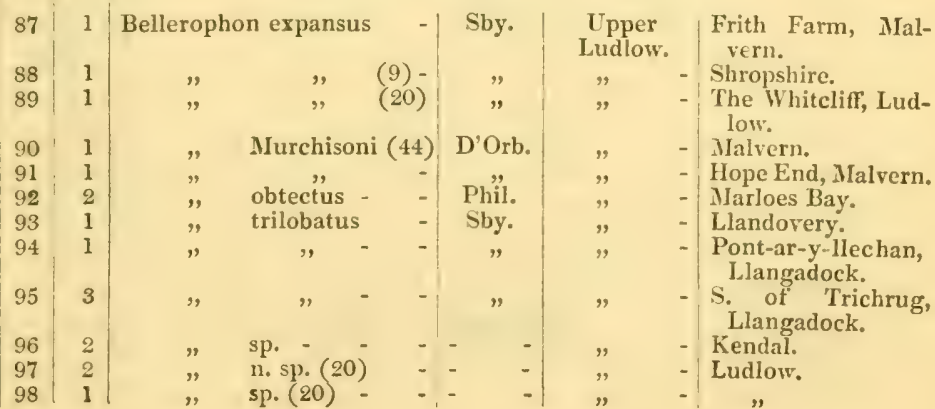




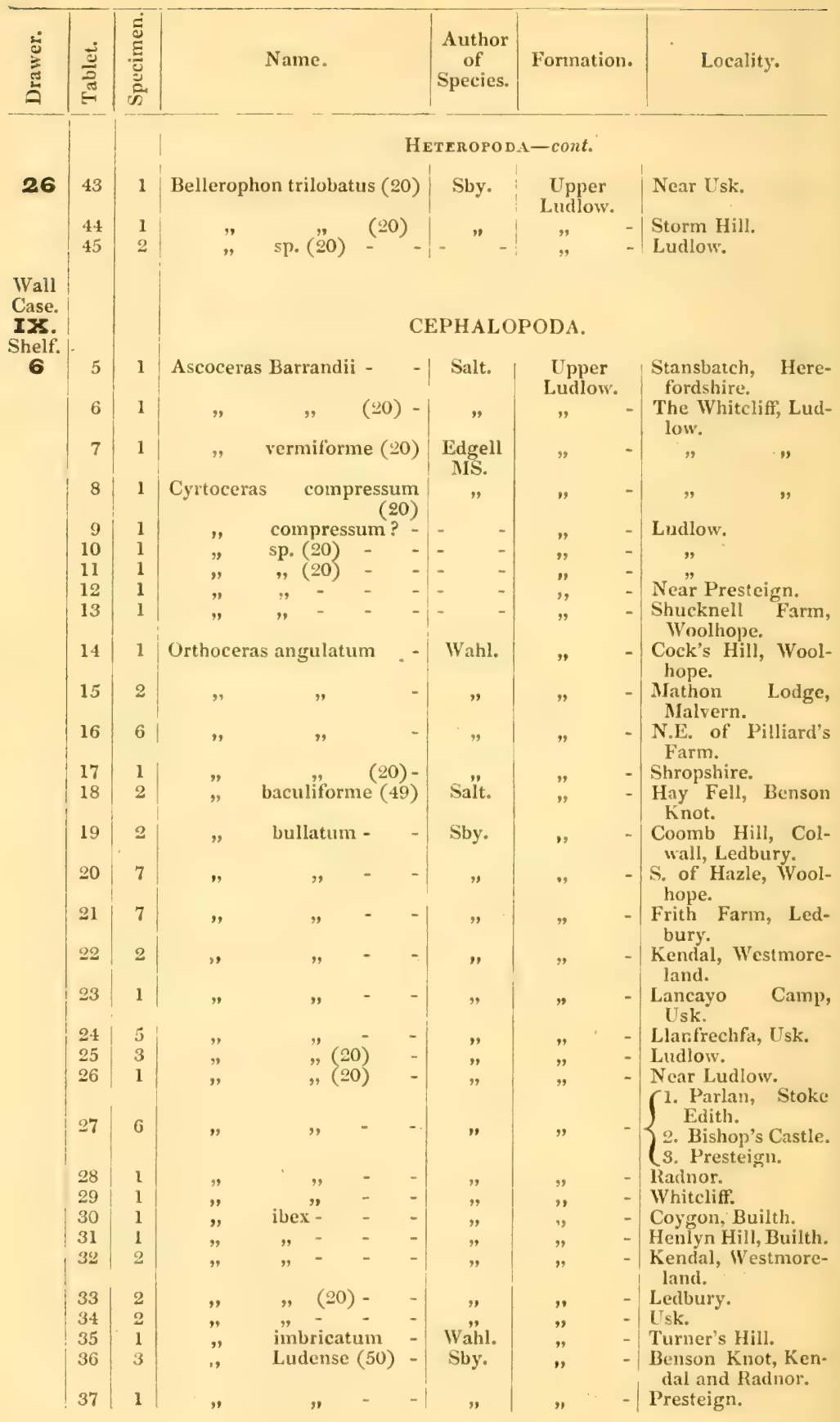




\begin{tabular}{|c|c|c|c|c|c|c|c|c|}
\hline 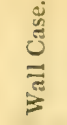 & है & $\frac{\text { हूं }}{\text { हूँ }}$ & & Name. & $\begin{array}{c}\text { Author } \\
\text { of } \\
\text { Species. }\end{array}$ & Formation & & Locality. \\
\hline rx. & & & \multicolumn{6}{|c|}{ Cerialopona-cont. } \\
\hline \multirow[t]{24}{*}{$\begin{array}{c}\text { Shelf: } \\
6\end{array}$} & 38 & 5 & Orthoceras & Maclareni & Salt. & $\begin{array}{l}\text { Upper } \\
\text { Ludlow. }\end{array}$ & & $\begin{array}{l}\text { N. Esk Quarry, } \\
\text { Pentland Hills, } \\
\text { (Carlops, Peebles). }\end{array}$ \\
\hline & 39 & 1 & " & "' & 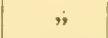 & " & - & 2 $" \quad n$ \\
\hline & 40 & 1 & " & " $\quad(20)$ & $"$ & - $"$ & - & Frith Farm \\
\hline & & & " & !erelegans & " & " & & $\begin{array}{l}\text { Frith Farm, Mal- } \\
\text { vern. }\end{array}$ \\
\hline & 42 & 2 & " & " & " & $"$ & - & Usk Castle. \\
\hline & 43 & 1 & $"$ & $(20)$ & " & $"$ & - & $\begin{array}{l}\text { The Whitcliff, Lud- } \\
\text { low. }\end{array}$ \\
\hline & 44 & 4 & " & subgregarium - & $\mathrm{M}^{6} \mathrm{Coy}$ & Bone Bed & - & $\begin{array}{l}\text { Newton Lane, } \\
\text { Kington. }\end{array}$ \\
\hline & 45 & 4 & " & subundulatum - & Portl. & $\begin{array}{l}\text { Upper } \\
\text { Ludlow. }\end{array}$ & & $\begin{array}{l}\text { Mathon Lodge, } \\
\text { Malvern. }\end{array}$ \\
\hline & 46 & 1 & " & " $\quad(20)$ & " & $"$ & - & Mocktree. \\
\hline & 47 & $\frac{1}{5}$ & " & , (20) & Phil & " & - & ? \\
\hline & 48 & 5 & "• & textile & PIII. & ", & - & $\begin{array}{l}\text { Freshwater East, } \\
\text { S. side. }\end{array}$ \\
\hline & 49 & 1 & " & torquatum (5) & Munst. & & - & Helme Foot. \\
\hline & 50 & 1 & ", & tracheale & Sby. & Tilestones & & $\begin{array}{l}\text { Horeb Chapel, } \\
\text { Llandovery. }\end{array}$ \\
\hline & 51 & 2 & " & $"$ & " & $\begin{array}{l}\text { Upper } \\
\text { Ludlow. }\end{array}$ & & Llandovery, Gadoc. \\
\hline & 52 & I & " & (20) & " & , & - & Llechciawdd. \\
\hline & 53 & 1 & $"$ & $"$ & " & Bone Bed & - & $\begin{array}{l}\text { Newton Lanc, } \\
\text { Kington. }\end{array}$ \\
\hline & 54 & 1 & $"$ & n. sp. & - & $\begin{array}{l}\text { Upper } \\
\text { Ludlow. }\end{array}$ & & $\begin{array}{l}\text { Lower Quarry, } \\
\text { Deerhope, Pent- } \\
\text { lands. }\end{array}$ \\
\hline & 55 & 1 & $"$ & sp. & - & ", & - & Blaenau, Builth. \\
\hline & 56 & 1 & $"$ & , & - & , & - & Presteign. \\
\hline & 57 & 1 & Phragmoce & eras nautileum & Sby. & " & - & Near Presteign. \\
\hline & 58 & 1 &, & " & ," & ", & - & $\begin{array}{l}\text { Upper Headlands, } \\
\text { Bishop's Castle. }\end{array}$ \\
\hline & 59 & 1 & , & sp. $\quad-$ & - & " & - & $\begin{array}{l}\text { N. Esk Reservoir, } \\
\text { Pentland Hills. }\end{array}$ \\
\hline & 60 & 1 & Tretoceras & bipartitum (20) & $\begin{array}{l}\text { Edgell } \\
\text { MS. }\end{array}$ & " & - & Horeb Chapel. \\
\hline & 61 & 2 & " & semipartitum & Sby. & ", & - & Llanfrechfa, Usk. \\
\hline
\end{tabular}

CRUSTACIA TROM IUDLOW ROCKS.

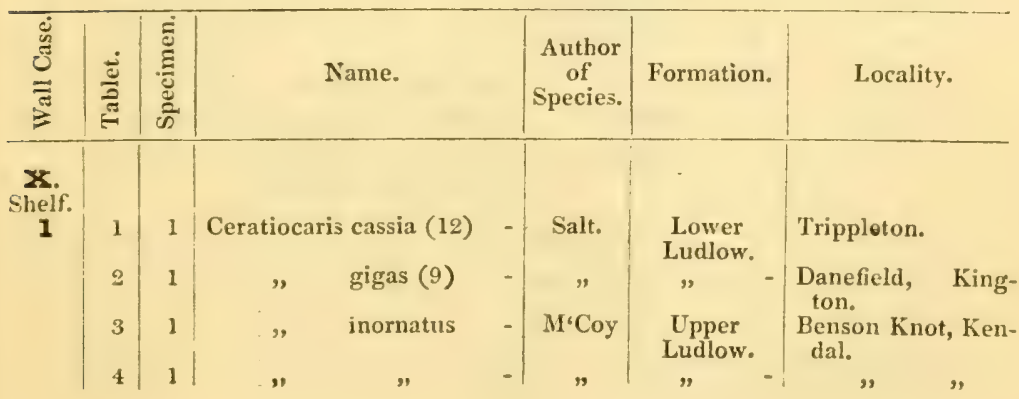




\begin{tabular}{|c|c|c|c|c|c|c|}
\hline 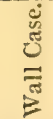 & 起 & $\frac{\tilde{0}}{\tilde{c}}$ & Name. & $\begin{array}{c}\text { Author } \\
\text { of } \\
\text { Species. }\end{array}$ & Formation. & Locality. \\
\hline
\end{tabular}

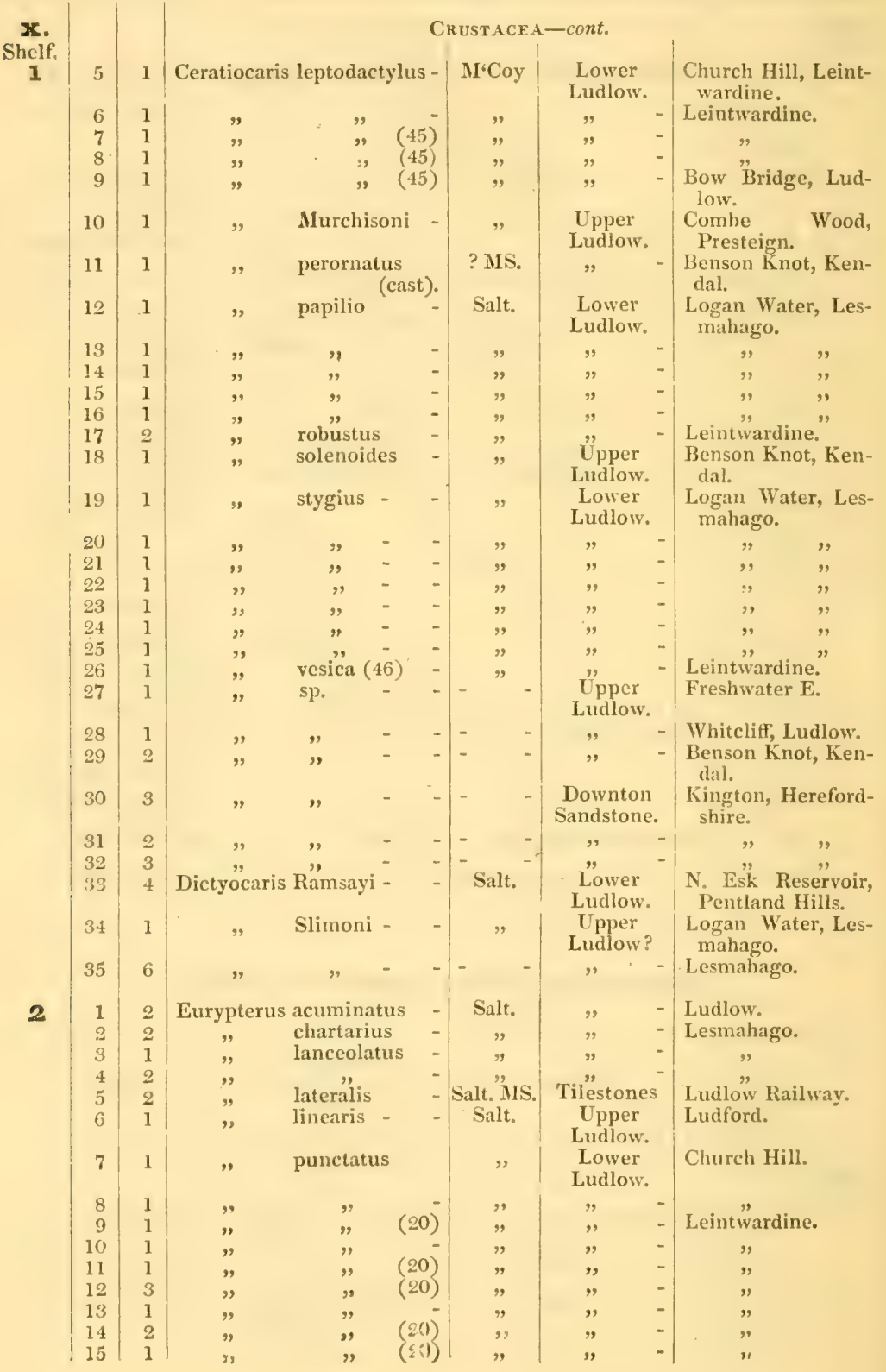




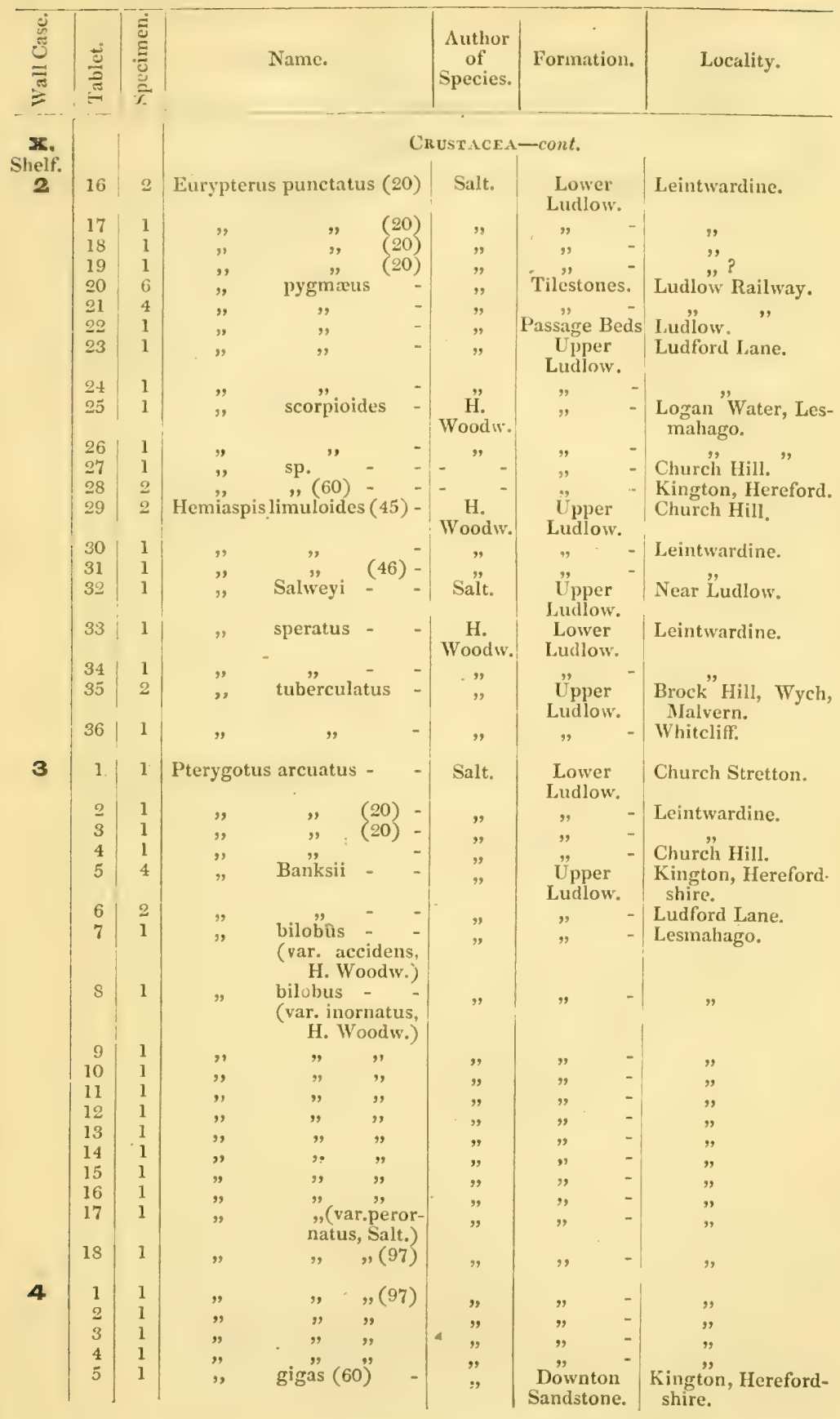


CRUSTACEA FROM LUDLOW ROCKS.

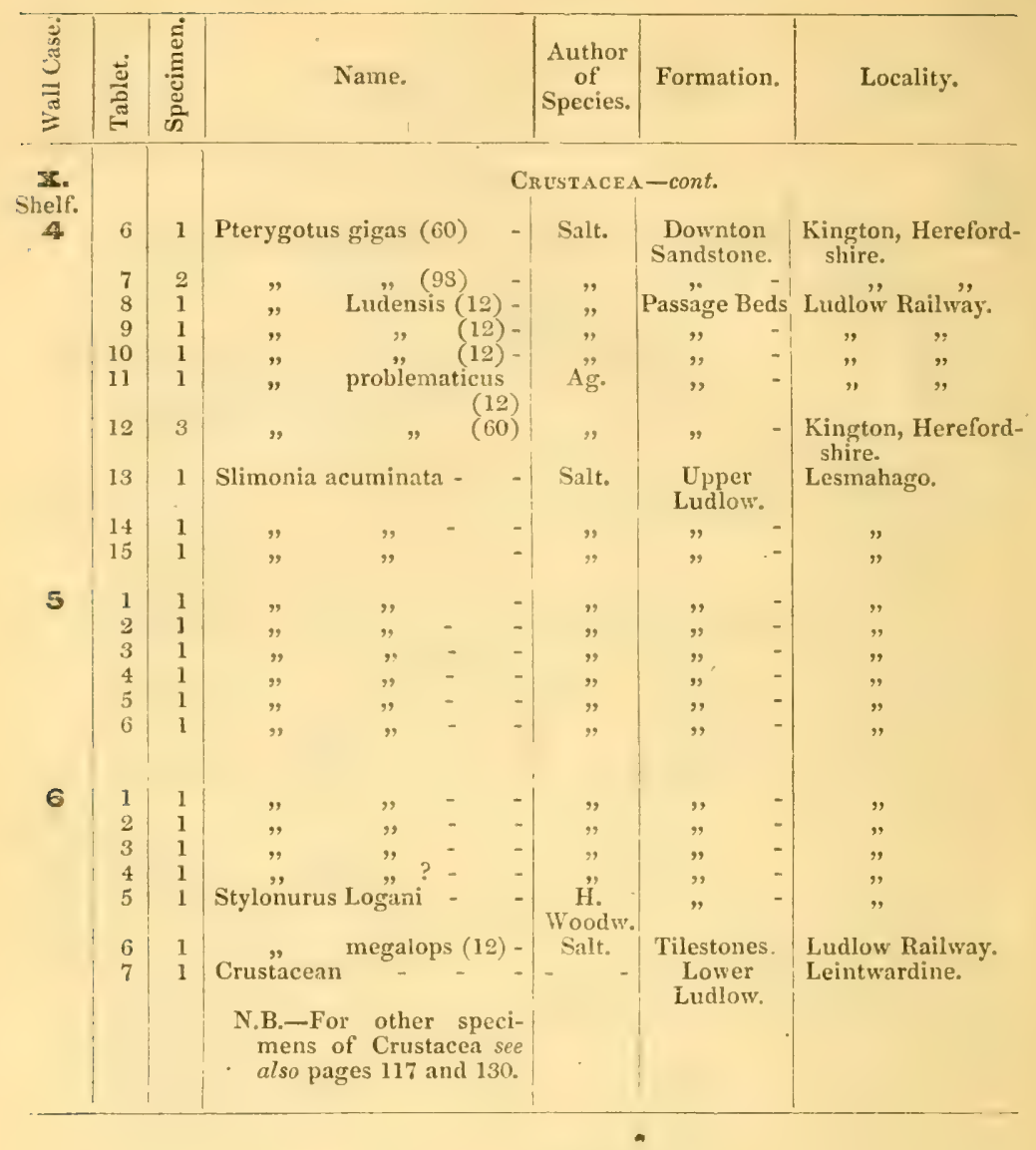

\section{ON DON :}

Printed by George E. Erre and William Spottrswoode,

Printers to the Queen's most Excellent Majesty.

For Her Majesty's Stationery Office.

$$
\text { [15929.-500,-3/78.] }
$$




$$
\text { . }
$$ 



\section{Date D1}

277 tecec 50

\section{AUG 101972}


